ORNL-6214

Distribution Category UC-34c

Physics - Nuclear

ORNL--6214

DE86 007072

\title{
ENGINEERING PHYSICS AND MATHEMATICS DIVISION PROGRESS REPORT FOR PERIOD ENDING JUNE 30, 1985
}

\author{
F. C. Maienschein, Director
}

\section{Date Published - February 1986}

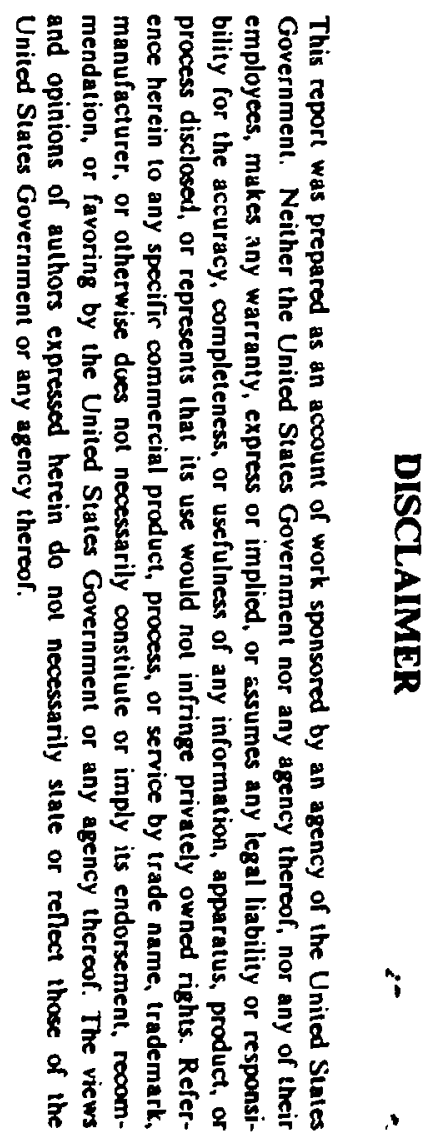

Oak Ridge National Laboratory

Oak Riúge, Tennessee 37831 operaled by

Martin Marietta Energy Systems, Inc. for the

U.S. DEPARTMENT OF ENERGY

under Contract No. DE-AC05-840R21400 
Reports previously issued in this series are as follows:

ORNL-2081
ORNL-2389
ORNL-2609
ORNL-2842
ORNL-3016
ORNL-3193
ORNL-3360
ORNL-3499, Vok. I and II
ORNL-3714, Vob. I and II
ORNL-3858, Voks. I and II
ORNL-3973, Vols. I and II
ORNL-4134
ORML-4280
ORNL-4433
ORNL-4592
ORNL-4705
ORNL-4800
ORNL-4902
ORNL-4997
ORNL-5101
ORNL-5280
ORNL-5504
ORNL-5725
ORNL-5897, Vok. I and II
ORNL-6030

Period Ending September 10, 1956 Period Eudies September 1, 1957 Period Ending September 1, 1958 Periad Eading September 1, 1959 Period Eviting September 1, 1960 Period Exting September 1, 1961 Period Endins September 1, 1962 Period Ining Anget 1, 1963 Period Enting Acrot 1, 1964 Period Enth Arat 1, 1965 Period Enting May 31, 1966 Period Earing May 31, 1957 Period Dantes May 31, 1968 Period Eading May 31, 1969 Period Entine May 31, 1970 Period Extien May 31, I971 Period Eading Mny 31, 1972 Period Eading May 31, 1973 Period Euling Ariget 31, 1974 Period Endias October 31, 1975 Period Eudte Felmary 28, 1977 Period Extion Novender 30, 1978 Period Eadieg Norember 30, 1980 Period Endine May 31, 1982 Period Ending December 31, 1983 


\section{PREFACE}

\section{F. C. Maienschein}

The progiess reports of the Engineering Physies and Mathematics Division (formerly the Engineering Physics Division) continue to consist principally of a collection of abstracts of journal articles, 1sports, and oral presentaiions describing research performed within or for the division. In addition, the progress reports inciude several appendices which give information about the scientific and professional activities of the division members and list division publications, paper presentations, and seminars.

This particular progress report differs somewhat from ous previous progress reports in that it is organized according to the eight major groups 'f the division rather than by subject matter. Thus, the report has eight sections identified as follows:

\section{Nuclear Data Measurements and Evaluations}

2. Systems Analysis and Shieldirg

3. Applied Physics and Fusion Reactor Analysis

4. Mathematical Modeling and Intelligent Control

5. Reliability and Human Factors Research

6. Applied Risk and Decision Analysis

7. Information Analysis and Data Management

8. Mathematical Sciences

The reader will recognize the similarity of several of the section headings with those in past reports, which is to be expecied since, in general, the various groups work in different research areas, some of which have been highlighted in earlier reports.

With this reorganization of the report, the gioup leaders have bern given the opportunity to introduce the sections with brief overviews of the work performed within their individual groups. In asking them to provide such overviews, we have not limited them tn the "period ending" date giver. on the cover page of this report nor to the subject matter covered by the abstracts included in their sections. As a result, the introductions to the sections may include discussions of work performed but not published during the reporting period, as well as work continued or initiated after the reporting period. In contrast, the abstracts comprising each section are of reports or journal articles which were published or accepted for publication before June 30, 1985, and of oral presentations that were scheduled before that date. We have not, however, included abstracts that appeared in the last progress report prior to their publication. Neither have we inciuded a few abstracts of reports identified as "Applied Technology." 
As is always the case, the division has undergone some changes in direction since the last reporting periud. The major change has resulted from the addition of the Mathematical Sciences Group, which was transferred into the division in February 1984 from what was then the Computer Sciences Division (now renamed the Computing and Telecciamunications Division). This transfer prompted the name change of our own division and added three new research areas: applied mathematics, computer science, and statistics. In applied mathematics, the current emphasis is on moving-boundary and phase-change problems in collaboration with experts in other parts of ORNL, and this work is expected to continue. Mush of the computer science effort is now focusing on the use of parallel processors, and in support of this work a hypercube computer with 64 processers has recently been installed. Finally, the group's traditionally strong program in statistical research continues to support programs related to biological and health effects; however, the emphasis is switching somewhat toward computational statistics, with studies of the best directions for this work being coordinated with other DOE laboratories. The work of the Mathematical Sciences Group is summarized in the introduction to Section 8 and is represented by the 120 abstracts included there. Some history of the group is also presented.

Section 1 of this progress report, titled Nuctear Data Measurements and Evaluation, deacribes how one of the division's oldest programs of research. performed primarily at the Oak Ridge Electron Linear Accelerator (ORELA), retains an admirabie continuity even as the kerel of support decreases owing to dramatic changes in the U.S. fission reactor program. Two remarkable developments iave occurred in this program which promise the availability of greatly improved nuclear data. First, the output of the ORELA pulsed neutron source recently has been significantly increased (by a factor of two of three), equaling the highest outputs ever achieved in the past, and recent insights into electron capture in the accelerator and the removal of dark currents from the electron guns offer a realistic hope for maintaining this performance. Second, a substantial barrier to further progress in precision measurements of the cross sections for fissile materials has been removed by the resolution of apfarent discrepancies among the measurements for ${ }^{236} \mathrm{U}$ throughout the world. This breakthrough is discussed in more detail in the introduction to Section 1.

The Systems Analysis and Shielding Group, whose work is reported in Section 2, also includes one of the oldest programs in the division: the development and testing of transport methods and cross-section data for application to the design of reactor shields. In recent years the experimental (testing) phase of this program, which has essentially all been performed at the ORNL Tower Shielding Facility (TSF), has been undersupported, but shielding studies at the TSF have now been revived through a joint program with Japan. In spite of uncertainties abcut the shape that future liquid-metal-ceoled reactors (LMRs) may assume in the United States, it is possible to plan effectively and carry out relevant experiments at the TSF to validate more efficient and less costly shielding materials and shield designs for new LMRs, including those being developed by the Japancse.

In the transport methods area, we have now accomplished the development of a threedimensional discrete ordinates method (called TORT, after years of planning. This development was primarily supported by the Defense Nuclear Agency, but its eventual application to other programs is inevitable. As is well known by those familiar with radiation transport calculations, the two-dimensional discrete ordinates code DOT that was developed within our division in carlier years has been the "work-horse" method, and three-dimensional results have had tc be obtained by Monte Carlo methods.

Another area covered in Section 2 is reactor core analysis. With the curtailing of U.S. fisaion reactor programs, the work reported reflects the past more than the present or future; however, the Oak Ridge National Laboratory Institutional Plan, October 1985, makes the clear asumption that a U.S. energy crisis, beginning with a shortage of liquid fuels, will occur within the next five years and a marked revival in reactor development and presumably in reactor analysis -- will iake place. 
An imprintant exception of division work in this area has been the design of a reactor with the highest possi "is thermal-neutron flux for use in the propusad Center for Neutron Research (CNR). which would ie a national fs jility for studies of condensed matter ind related topics.

Other $₹ 13^{2}$ ysis activities eported in this section include studies of transport theory. A:tention is now focavinx on nonlinear physical systems, including transport phenomena, as well as reactor kileties and nijid dynamies. A center is bring started for interaction with nther ORNL scientists and with visiting scientists who a,e worting on problens of nonlinear phenomena. Twere is current zollaborative research in rargaetohyarodynamics, as in !usion and solar systems, and in the physics If interactions of heary ions with real surfacs.

Section 2 s'so describes studies on nuriear weapons effects that fall under two differcat programs: one supported by DOE to determi, 2 the radia:ion doses delivered to victims at Hiroshima and Nagzsaki, and another :?:pported by the coderel Emergency Management Agency (FEMA) to supply iandibooks on radiation protection to : the general public. The second of these twu programs was trans!erred into wur division during the : itiod covered by this report.

Sertion 3. titled Applied Physics ar i Fusion Reactor Analysis, covers our fusion reactor research and car continuing reazarch relatest to high-el,ergy particle accelerators. While the fusion work repozted here includes a aumber of exneriments, the program has now been reoriented in the sense that integral experin:ents have been $r t$ aside juing to financial constraints. Continuing studies of frision rezzor shiels: and of neutir n economius in blanket regions will largely consist of the analyses of experiments to be sarried out i: ine eicgan: Japenese facilities.

Our high-energy resesrch continues ia a smail tut efficient support group for the analysis of shieids for zew high-enetgy arcelerators nd for $q$ :antitative analysis of the performance of the massive instruments useci as detectors a those acaelerators. Recent calculations have led to widespread reesduation c: :he energy re iution to ise expected from new and expensive detectors (calorimeters ountaining uranium). A se 'sus and setermined proposal to expand our high-energy research as direct collabo: ztors in neutrin. meast p'snients was not accepted in a year of tight financial constraints, in spite o' the favorable Ieviews i is proposal received.

Section 4 describes the wi:rk of the inther:atical Modeling and Intelligent Control group. It firs: addresses our contin: ing studies on :.,e possitility of intelligent machines, which have attracted the attention of experts $i$ : the artificial : :elliger:e and robotic communities. A second hypercube computer was obtained $l y$ the division i, this rork, in which a current emphasis is on advanced parallel-proceasing progrim mining technipries whici: will accommodate the relatively unstructured environments encounterc: in the contro bi a rrizot (and may also have important applications for the Department of Defei e). Work on iie deve spinent of a series of mobile robots for autonomous operation also continues The robots, flich a: gradually increasing in sophistication, are being adapted with low-mass : gh-compliance urmis ar.d manipulators at a pace largely determined by the ava lability of capital fu iding.

Another series of " I ties reported in Sectirn 4 has demonstrated how national laboratories can provide an expertise ar : sentinuity of efont fo. long and difficult programs of broad interest. Calculations spensored mu ily by the Elect:ic Pover Resessch Institute (EPRI) have determined with unprecedented accurac; the fluences of nestro $\mathrm{L}$, inside the pressure vessels of light water reactors. The interest in the nus ices arises from the an:icipated damage to the vessel by long-term irradiation. This damage might result in shortened lives of $t$ actors which have particular sensitivity to radiation damage beca'sse of materials that a.e incorp rated into their welds. This program has extended over a period of at least seven years, and only by virtue of its continuity has it been pousible to obtain the defin ive results which will : end to g ide all future evaliation of pressure vessel damage in light water ieactors. 
Other mathematical modeling efforts described in Section 4 are related to improving reactor physics analysis methods, evaluating proposed radioactive waste isolation systems, and applying sensitivity techniques to the $\mathrm{CO}_{2}$ climate problem (now discontinued). The program to assess the performance of waste isolation systems is a new area for the division, its objective being to provide basic research support to the DOE Waste Management Programs and the Civilian Radioactive Waste Management Office. Another program that continues to be active within this group but is not represented in Section 4 by abstracts is energy economic modeling analysis for fossil fuel systems. The goal of this work is to provide to DOE information that is independent of that received from the oil companies. (Paper 3.15 describes some of the past work in this program.)

In a separate effort closely related to several of the programs within this group, wort on the GRESS method continues. GRESS is a method for automatically obtaining the necessery derivatives to apply adjoint perturbation theory, and it is expected to be utilized in uncertainty analya: in many fields It is currently being applied in both our robotics work and in the waste isolation program.

Section 5, Reliability and Human Factors Research, rexorts work in three general areas: system (hardware) reliability, human performarce measurement, and personnel qualification and training. During the reporting period substantial portions of this program were funded by the Nuctear Regulatory Commission, which, however, is de-emphasizing research in this area. One portion of the program, the operation of the Centralized Reliability Data Organization (CREDO), was funded by DOE and this effort will b: expanded under the joint sponsorship of DOE and Power Reactor and Nuclear Fuel Development Corporation (PNC) of Japan. CREDO is a data base for the reliability of LMR components, and in order to make best use of the expanded data base, it will be necessary to give increasing attention to the analysis of the collected information so that it can be applied in the most 1..eaningf I manner to the design of future reactors.

One of the most significant accomplishments of the NRC program was the several-year development of the computer model MAPPS to predict the reliability of nuclear plant maintenance personnel. The model has wide application potential in both nuclear and non-nuclear fields. Similarly, automated tools developed for qualifying and training nuclear plant personnel can be adapted to other disciplines.

Sextion 6, Applied Risk and Decision Analysis, describes a program largely funded by the NRC to study various risks to nuclers power plants, that is, risks posed by floods, fires, various types of accidents, etc. Resently, this program has included the development of a PC-based automated information management system (PRISIM) which allows rapid access to plant-specific risk data and which has attracted great interest within NRC.

Most of the risk analysis work is performed by subcontractors under the management of the division; however, one large NRC project which has just been completed ipvolved both management and research by division personnel, as well as research by members of other ORNL divisions and several outside organizations. Called the PTS project, it consisted of the evaluation of the "pressurized thermal shock" phenomenon for the pressure veseels of three nuclear power plants that have operated for many years. The degree of cooperation between ORNL, other organizations, NRC, and the utilities during this project appears to have been unprecedented, and fortunately the result of the study was favorable in the sense that the threst of life shortening for the reactors under study is greatly alleviated. It has been eatimated that this development will ane the nuclear induztry billions of dollars by precluding the necessity for costly retrofits during the nominal life of a power plant. 
Section 7, Information Analysis and Data Management, reports on the work of the Engineering Physios 'nformation Centers (EPIC). Currently two centers z.e being operated: the Radiation Shielding In! ormation Center (RSIC), which is in its 22r.a year and interacts with organizations all over the world, and the Tectnical Data Management Center (TDMC), which was eatablished by the NRC in 1976. RSIC collects, evaluates, and distributes bibliograhic information, data sets (particularly cross-section libraries), and computer codes vital to the shielding community. It also participates in establishing shielding benchmark problems and standards and provides problem assistance to requesters. TDMC does much of the same type of wort for NRC programs, with emphasis on computer codes: the center provides complete input information and real-time advice so that the user can implement the methods in a meaningful manner.

A review of the various sections of this report will reveal a wide diversity of interests not only betwosn the eight groups making up the division but also within the individual groups themselves. Scill there is considerable interaction among the groups as they frequently utilize the same "engineering physics and mathematics" methods. In fact, much of our present research program has resulted from the continuing realization that techniques and methods we have developed in the past, combined with and adapted to a fast-changing computer technology, can be applied in new and exciting areas of investigation. We remain confident that such applicaisons will continue as an essential part of the programs of the Department of Energy and related agencies. 


\section{TABLE OF CONTENTS}

PREFACE

\section{Section 1. NUCLFAR RATA MEASUREMIENTS AND EVALUATKCN}

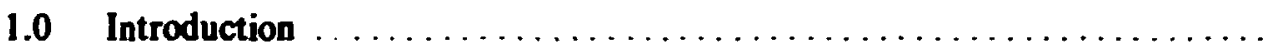

\section{Experimeatal Resolts}

1.1. High-Resolution Structural Material $(n, x \gamma)$ Production Cross Sections for $E_{n}$ from 0.2 to $40 \mathrm{MeV}$

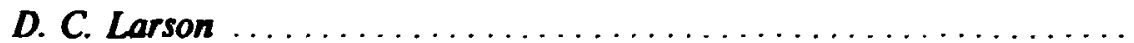

i.2. Fast Neutron Inelastic Scattering from ${ }^{53} \mathrm{Cr}$

J. K. Dickens and D. C. Larson

1.3. Measuremerts of the Energy Dependence of Prompt Neutron Emission from ${ }^{233} \mathrm{U},{ }^{235} \mathrm{U},{ }^{239} \mathrm{Pu}$, and ${ }^{241} \mathrm{Pu}$ for $E_{n}=0.005$ to $10 \mathrm{eV}$ Relative to Emission from Spontaneous Fission of ${ }^{252} \mathrm{Cr}$

$\boldsymbol{R}$. Gwin, $\boldsymbol{R}$. $\boldsymbol{R}$. Spencer, and $\boldsymbol{R}$. W. Ingle

1.4. Measurement of the Energy Dependence of Prompt Neutron Emission from ${ }^{233} \mathrm{U},{ }^{235} \mathrm{U}$, and ${ }^{239} \mathrm{Pu}$ for $E_{n}=0.0005$ to $10 \mathrm{MeV}$ Relative to Emission from Spontaneous Fission of ${ }^{252} \mathrm{Cf}$

$\boldsymbol{R}$. Gwin, $\boldsymbol{R} \boldsymbol{R}$. Spencer, and $\boldsymbol{R} \boldsymbol{W}$. Ingle

1.5. Measurements of the Neutron Fission Cross Sections of ${ }^{235} \mathrm{U}$ $\left(E_{n}=0.01 \mathrm{eV}\right.$ to $\left.30 \mathrm{keV}\right)$ and ${ }^{239} \mathrm{Pu}\left(E_{n}=0.01\right.$ to $\left.60 \mathrm{eV}\right)$

$R$. Gwin, R. R. Spencer, R. W. Ingle, J. H. Todd, and S. W. Scoles ...

1.6. Subthreshold Fission Cross Section of ${ }^{240} \mathrm{Pu}$ and the Fission Cross Sections of ${ }^{235} \mathrm{U}$ and ${ }^{239} \mathrm{Pu}$

L. W. Weston and J. H. Todd

1.7. Nature of the Coupling in Subthreshold Fission of ${ }^{238} \mathrm{~Np}$

G. F. Auchampaugh, M. S. Moore, J. D. Moses, R. O. Nelson,

R. C. Extermann, C. E. Olsen, N.W. Hill, and J. A. Harvey 
1.8. Neutron-Induced Fission Cross-Section Measurements of ${ }^{244} \mathrm{Cm}$, ${ }^{246} \mathrm{Cm}$, and ${ }^{248} \mathrm{Cm}$

H. T. Maguire, Jr., C. R. S. Stopa, R. C. Block, D. R. Harris,

$R$ E. Slovacek, J. W. T. Dabbs, R. J. Dougan, $R$ W. Hoff, and

$\boldsymbol{R}$ W. Lougheed

1.9. Determination of Unbound States in ${ }^{35} \mathrm{~S}$ from Neutron Total and Capture Cross-Section Measurements of ${ }^{34} \mathrm{~S}$

R. F. Carlion, W. M. Good, J. A. Harvey, R. L. Macklin, and

B. Castel

1.10. Neutron Capture and Total Cross Sections for ${ }^{48} \mathrm{Ca:}$ : Astrophysical Implications

R. F. Carlion, J. A. Harvey, N. W. Hill, and R. L. Macklin.

1.11. ${ }^{58} \mathrm{Ni}+n$ Transmission. 'apture and Differential Elastic Scattering

Data Analysis in the Resunance Region

C. M. Perey, F. G. Perey, I. A. Haney, N. W. Hill, and

R. L Macklin

1.12. Measurements of the Neutron Transmission and Capture Cross Sections in ${ }^{204} \mathrm{~Pb}$

D. J. Horen, R. L. Macklin, J. A. Harvey, and N. W. Hill

1.13. Neutron Capture by ${ }^{3 !} \mathbf{P}$

R. L. Macklin and S. F. Mughabghab

1.14. Resonance Neutron Capture by ${ }^{35,37} \mathrm{Cl}$

R. L. Macklin

1.15. Resonance Neutron Capture by ${ }^{39,41} \mathrm{~K}$

R. L. Macklin

1.16. Rescnance Neutron Capture by Manganese Below $2.5 \mathrm{keV}$

R. L. Macklin

1.17. Capture in the 1.15-keJ Iron Resonance

L. W. Weston and J. H. Todd

1.18. Neutron Capture Measurements on Radioactive ${ }^{93} \mathrm{Zr}$

R. L. Macklin

1.19. Neutron Capture Measurements on Fission Product Pelladium-107

R. L. Macklin

1.20. Neutron Capture Cross Sections and Solar Abundances of ${ }^{160.161}$ Dy, ${ }^{170,171} \mathrm{Yb},{ }^{175,176} \mathrm{Lu}$, and ${ }^{176,177} \mathrm{Hf}$ for the s-Process Analysis of the the Radionuclide ${ }^{176} \mathrm{Lu}$

H. Beer, G. Walter, R. L. Macklin, and R. J. Patchett 
1.21. The ${ }^{163} \mathrm{Dy}-{ }^{163} \mathrm{Ho}$ Branching: An s-Process Barometer

H. Beer, G. Walter, and R. L. Macklin

1.22. $198,199,200,201,202,204 \mathrm{Hg}_{\mathrm{g}}(n, \gamma)$ Cross Sections and the Termination of s-Process Nucleosynthesis

H. Beer and $\mathbf{R}$ L Mack! in

1.23. Neutron Capture Cross Section of Americium-243

$L, W$. Weston and J. H. Todd

1.24. s- and p-Wave Neutrons on ${ }^{30} \mathrm{Si}$ and ${ }^{34} \mathrm{~S}$ : Spherical Optical Model Analysis

R. F. Carlton, J. A. Harvey, and C. H. Johnson

1.25. Neutron, Alpha and Total Widths and Spin Assignments for Resonances in ${ }^{33} \mathrm{~S}+n$ from $10-400 \mathrm{keV}$

G. P. Coddens, M. Salah, J. A. Harvey, and N. W. Hill . . . . . . .

1.26. $d_{5 / 2}$-Single Particle Strength in ${ }^{48} \mathrm{Ca}+n$

J. A. Harvey, C. H. Johnson, R. F. Carlton and B. Castel

1.27. Single-Particle $2 d_{5 / 2}$ Strength in the ${ }^{48} \mathrm{Ca}+n$ Reaction

J. A. Harvey, C. H. Johnson, R. F. Carlton, and B. Castel

1.28. Parity Dependence of the Level Densities of ${ }^{53} \mathrm{Cr}$ and ${ }^{55} \mathrm{Cr}$ at High

Excitation

H. M. Agrawal, J. B. Garg, and J. A. Harvey . . . . . . . . . . . .

1.29. Optical Model Srattering Functions for Low Energy Neutrons on ${ }^{86} \mathrm{Kr}$

R. F. Carlton, J. A. Harvey, and C. H. Johnson.

1.30. Total Cross Sections for Low Energy Neutrons on ${ }^{86} \mathbf{K r}$

R. F. Cariton, C. H. Johnsom, and J. A. Harvey

1.31. Accurate Determination of the Parameters of the 292.4-eV Resonance of ${ }^{91} \mathrm{Zr}$ and the $301.3-\mathrm{eV}$ Resonance of ${ }^{96} \mathrm{Zr}$

M. M. Sa'ah, J. A. Harvey, N. W. Hill, A. Z. Hussein, and

F. G. Perey . . . . . . . . . . . . . . . . . . . . . . . . . .

1.32. Neutron Trarımission Measurement and Resonance Analysis of ${ }^{93} \mathrm{Zr}$ from 60 to $60100 \mathrm{eV}$

R. L. Macklin, J. A. Harvey, and N. W. Hill

1.33. Observation of Extremely Low s-Wave Strength in the Reaction ${ }^{136} \mathrm{Xe}+n$

B. Fogelberg, J. A. Harvey, M. Mizumoto, and S. Raman

1.34. Test of Fermi Gas Model Predictions of Level Density in ${ }^{137} \mathrm{Xe}$

B. Fogelberg, J. A. Harvey, M. Mizumoto, and S. Raman 
1.35. Neutron Total Cross Section of Pu-240 Below $6 \mathrm{eV}$ and the Parameters of the 1.056-eV Resonance

R. R. Spencer, J. A. Harvey, N. W. Hill, and L. W. Weston . . . . . .

1.36. Fission-Product Yields for Thermal-Neutron Fission of ${ }^{243} \mathrm{Cm}$ Determined from Measurements with a High-Resolution Low-Energy Germanium Gamma-Ray Detector

L. D. Merriman

1.37. Calculated Neutron-Induced Cross Suctions for ${ }^{63.65} \mathrm{Cu}$ from 1 to 20 $\mathrm{MeV}$ and Comparisons with Experiments

D. M. Hetrick, C. Y. Fu, and D. C. Larson

\section{Erabations and Reviews}

1.38. Calculated Neutron-Induced Cross Sections for ${ }^{63,65} \mathrm{Cu},{ }^{58,60} \mathrm{Ni}$, and ${ }^{52} \mathrm{Cr}$ from 1 to $20 \mathrm{MeV}$ and Comparisons with Experiments

D. M. Hetrick, C. Y. Fu, and D. C. Larson

1.39. Pairing Correction of Particle-Hole State Densities for Two Kinds of Fermions

C. $Y . F u$

1.40. Simplified Spin Cutoff Factors for Particle-Hole Level Densities in Precompound Nuclear Reaction Theory

C. $Y . F u$

1.41. Multilevel Analysis of the Low-Energy ${ }^{239} \mathrm{Pu}$ Cross Sections

G. de Saussure, R. B. Perez, and Roger Macklin

1.42. R-Matrix Analysis of the ${ }^{239} \mathrm{Pu}$ Neutron Cross Sections

G. de Saussure, R. B. Perez, and Roger L Macklin

1.43. Multilevel Analysis of the Low Energy ${ }^{239}$ Pu Cross Sections

R. B. Perez, G. de Saussure, N. M. Larson, and Roger Macklin

1.44. Report to the ${ }^{238} \mathrm{U}$ Discrepancy Task Force on SIOB Fits to the ORNL, CBNM, and JAERI Transmission Data

D. K. Olsen

1.45. Resolved Resonance Parameters for ${ }^{238} \mathrm{U}$ from 1 to $10 \mathrm{keV}$

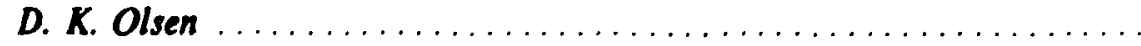

1.46. Status of the Parameters of the 1.15-keV Resonance of ${ }^{56} \mathrm{Fe}$

F. G. Perey

1.47. The Neutron Cross Section Standards Evaluations for ENDF/B-VI

A. D. Carlson, W. P. Poenitz, G. M. Hale, and R.W. Peelle 
1.48. Reports on Nuclear Data Activities for the DOE Nuclear Data Committee

D. C. Larson . . . . . . . . . . . . . . . . . . . . . . . . . . . .

1.49. Office of Basic Energy Sciences Program to Meet High Priority Nuctear Data Needs of the Office of Fusion Energy: Summary of Results 1976-1983

D. C. Larson and R. F. Haight

1.50. Improving Neutron Reaction Data Through LINAC-Based Flight-Time Spectrometry

R. W. Peelle

1.51. Summary Talk Covering Application-Oriented Sessions

$\boldsymbol{R}$. W. Peelle . . . . . . . . . . . . . . . . . . . . . . . . . .

Techimpes and Facilitios

1.52. Uncertainty Propagation from Raw Data to Final Resvits

N. M. Larson.

1.53. Updater Users' Guide for SAMMY: Multilevel R-Matrix Fits to Neutron

Data Using Bayes' Equations

N. M. Larson.

1.54. Analysis of Nestron Data in the Resonance Region Via the Computer Code SAMMY

N. M. Larson

1.55 User's Guide for ALEX: Uncertainty Propagation from Raw Data to Final Results for ORELA Transmission Measurements

N. M. Larson.

1.56. Majority Logic NE-1 10 Detectors for $\mathrm{keV}$ Neutrons

N. W. Hill, J. A. Harvey, D. J. Horen, G. L. Morgan, and

R. $R$. Winters

1.57. An NE-213-Scintillator-Based Neutron Detection System for Diagnostic Measurements of Energy Spectra for Neutrons Having Energies $\geqslant 0.8$ MeV Created During Plasma Operations at the Princeton Tokamak Fusion Test Reactor J. K. Dickens, N. W. Hill, F. S. Hou, J. W. McConnell, R. R. Spencer, and $F$. Y. Tsang

1.58. A Preliminary Study of Possible ORELA Replacement Options

D. K. Olsen, J. A. Martin, and D. J. Horen

1.59. Calculations Pertaining to the Design of a Prebuncher for a 150-MeV

Electron Linear Accelerator III. Comparisons with Experimental Data $R$ G. Alsmiller, Jr., F. S. Alsmiller, and T. A. Lewis 
1.60. ORELA Flight Path 1: Determinations of Its Effective Length vs

Energy, Experimental Energies, and Energy Resolution Function and

Their Uncertainties

D. C. Larsom, N. M. Larson, and J. A. Harvey . . . . . . . . . . . 29

1.61. Laser Measurem $₫$ nts of Distances from the ORELA Neutron Target to Experiment Stations Along Flight Paths 1 nd 6

D. C. Larsun, N. M. Larson, J. A. Harvey, F. G. Perey, D. E. Pierce, and $R$. Seals

\section{Section 2. SYSTEMS ANALYSIS AND SHIELDING}

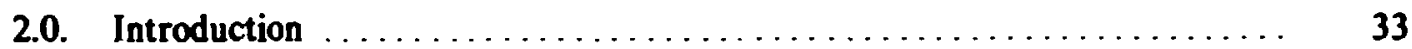

\section{Radiation Traseport Methods and Data}

2.1. The MORSE Monte Carlo Radiation Transport Code System

$M$. E Emmett ............................... 41

2.\%. MORSE-CGA: A Monte Carlo Radiation Transport Code with Array Geometry Capability

M. B. Emmett

2.3. Applications Guide to the MORSE Monte Carlo Code

S. N. Cramer

2.4. Variance Reduction Methods Applied to Deep-Penetration Monie Carlo Problems

S. N. Cramer and J. S. Tang .

2.5. Applications Guide to the RSIC-Distributed Version of the MCNP Code (Coupled Monte Carlo Neutron-Photon Code)

$\boldsymbol{S} . \boldsymbol{N}$. Crar:er . . . . . . . . . . . . . . . . . . . . . . . . . . . . . .

2.6. Applications Guide to the RSIC-Distributed Version of the KENO-V Code

S. N. Cramer

2.7. Monte Carlo Techniques for Analyzing Deep-Perietration Problems

S. N. Cramer, J. Gonnord, and J. S. Hendricks

2.8. A Method for Coupling Two-Dimensional to Three-Dimensional Discrete Ordinates Calculations

J. I. Thompson, M. B. Emmett, W. A. Rhoades, and

H. L. Dodds, Jr. 
2.9. The Linear Nodal Method for Shielding Applications

I. L. Childs and W. A. Rhoades.

2.10. The Extensicn of the Linear Nodal Method to Large Concrete Building Calculations

R. L. Childs and W. A. Rhoades

2.11. Impact of ${ }^{57} \mathrm{Fe}$ on Neutron Penetration in Thick Sodium-Iron Shields

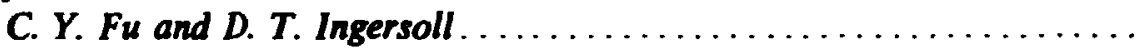

Radiation Shieldien Applications

2.12. Comparison of Candidate Shielding Materials for Space Power Reactor Applications

W. W. Engle, Jr. and D. E. Bartine ................... 45

2.13. Integral Neutron and Gamma-Ray Measurements of the Leakage. Flux from a Mockup of Little Boy

F. J. Muckenthcler.

2.14. Applications of the Three-Dimensional Oak Ridge Transport Code

W. A. Rhoades, R. L. Childs, M. B. Emmett, and S. N. Cramer ......

2.15. Analysis of the TORT Validation Expcriment

W. A. Rhoades, R. L. Childs, D. T. Ingersoll, and

F. J. Muckenthaler.

2.16. Analysis of Neutron Streaming in the HTGR Lower Support Regions

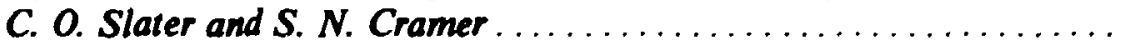

2.17. Analysis of Phase I of the HTGR Bottom Reflector and Core Support Block Neutron-Streaming Experiment

C. O. Slater

Reactor Analyses

2.18. A Theory of the Fission-Source-Driven Neutron Noise Field

F. C. Difilippo

2.19. Flux Synthesis for the On-Line Surveillance of Nuclear Power Plants

F. C. Difilippo, D. G. Cacuci, and B. A. Worley

2.20. Neutronic Calculations for a New High Flux Reactor

F. C. Difilippo, B. A. Worley, and D. R. Vondy

2.21. Stochastic Processes in a Subcritical Nuclear Reactor in the Presence of a Fission Source

F. C. Diflippo. 
2.22. Thermal Reactor Design for a Neutron-Scattering Source Facility

B. A. Worley, F. C. Difilippo, and D. R. Vondy ............. 50

2.23. Modular Gas-Cooled Reactor Heat Transfer Mechanisms

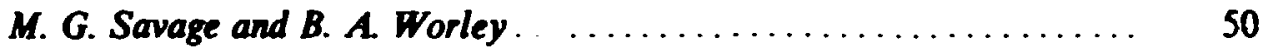

2.24. A One-Dimensional Mrdeling of Radial Heat Removal During

Depressurized Heatup Transients in Modular Pebble-Bed and

Prismatic High-Temperature Gas Cooled Reactors

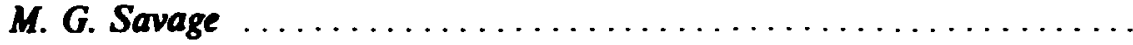

2.25. Application of Variational-Heuristic Learning Methods to Reactor

Physics: A New Concept in Artificial Intelligence

T. L. King, R. B. Perez, D. G. Cacuci, R T. Wood, and

E. L. Machado

2.26. Dete-ministic Sensitivity Analysis of Two-Phase Flow

Systems: Forward and Adjoint Methods

D. G. Cacuci, E. Wacholder, S. Kaizerman, and N. Tomerian

2.27. An Exact Sensitivity Analysis of a Simplifie' Transient Two-Phase

Flow Problem

E. Wacholder, S. Kaizerman, N. Tomerian, D. G. Cacuci

2.28. Adjoint Sensitivity Analysis of a Thermal-Hydraulic System Undergoing

Phase Change Due to Boiling Transition

D. G. Cacuci, E. Wacholder, S. Kaizerman, and N. Tomerian

2.29. Nonlinear Dynamics and Stability of Boiling Water Reactors. I:

Qualitative Analysis

J. March-Leuba, D. G. Cacuci, and R. B. Perez

2.30. Nonlinear Dynamics and Stability of Boiling Water Reactors. II:

Quantitative Analysis

J. March-Leuba, R. B. Perez, and D. G. Cacuci

2.31. Calculation of Limit Cycle Amplitudes in Commercial Boiling Water

Reactors

J. March-Leuba, R. B. Perez, and D. G.

2.32. A Stochastic Model to Monitor Mechanical Vibrations in Pressurized Water Reactors

R. T. Wood, J. March-Leuba, R. B. Perez, and F. J. Sweeney . . . . .

2.33. Benchmark Problem Solution: Two-Dimensional PWR Burntp Problem with Fuel Management Treating Two Cycles

D. $R$. Vondy 
xvii

2.34. THR-TH: A High-Temperature Gas-Cooled Nuclear Reactor Core Thermal Hydraulics Code

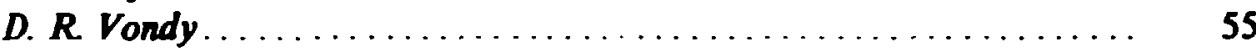

2.35. 2240-MW(th) High-Temperature Reactor Core Power Density Study

D. $R$ Vondy . . . . . . . . . . . . . . . . . . . . . . 56

2.36. A Multigrid Iteration Solution Procedure for Solving Two-Dimensional Sets of Coupled Equations

D. $R$. $V_{c}$ ndy . . . . . . . . . . . . . . . . . . . . . . . . 56

2.37. A Multigrid Iteration Solution Procedure for Solving Three-Dimensional Sets of Coupled Equations

D. $R$. Vordy . . . . . . . . . . . . . . . . . . . . . . 56

2.38. Regarding Overrelaxation for Accelerating an Iteration Process

D. $\boldsymbol{R}$ Vondy . . . . . . . . . . . . . . . . . . 57

2.39. The Depth-Charge Static and Time-Dependent Perturbation/Sensitivity System for Nuclear Reactor Core Analysis

2.40. Developing Nuclear Power for Space Applications

D. E. Bartine

2.41. Exact Analytic Solution for the Generalized Flux in One-Dimensional Cartesian Geometry

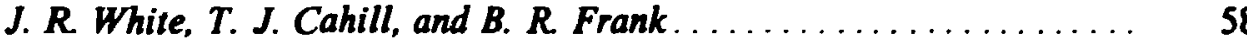

2.42. Evaluation of the DIF3D Nodal Option for HTGR Neutronic Applications B. $R$. Frank and J. R. White

2.43. Few-Group Three-Dimensional Hexagonal Geometry HTGR Benchmask Problem

B. R. Frank and J. R. White

2.44. inssessing Applications of Sauer's Method for Calculating Dancoff Factors

R. D. Timmerman

Analysis of Reactor Operating Data

2.45. The Analysis and Evaluation of Recent Operational Experience from the Fort St. Vrain HTGR

D. L. Moses and W. D. Lanning 
xviii

\section{Stadies of Nectear Weapons Effects}

2.46. Adjoint Transport Calculations for Sensitivity Analysis of the Hiroshima Air-Over-Ground Environment

B. L. Broadhead, D. G. Cacuci, and J. V. Pace III ............ 60

2.47. In the Shadow of Ground Zero

C. M. Haaland

60

2.48. A Griphical Method for Forecasting Radiation Exposure from MultiAged Fallout from Nuclear Weapons

C. M. Haaland

2.49. A Review of the Nuclear Winter Scenario

C. M. Haaland

2.50. Reply to Victor N. Evdokimoff

C. A. Haaland

61

2.51. Forecasting Radiation Exposure from Fallout Caused by Multiple,

Nonsimultaneous, Upwind Ground Bursts

C. M. Haaland

61

2.52. Snculd We Protect Ourseives from Nuclear Weapons Effects?

C. M. Haaland

2.53. A Simple Graphical Method for Forecasting Radiation Exposure from Multi-Aged Fallout from Nuclear Weapons

C. M. Haaland

2.54. Improving RADEF

R. N. Thurmer

\section{Mathematical Physics}

2.55. On the Spectral Decomposition of the Transport Operator with

Anisotropic Scattering and Periodic Boundary Conditions

V. Protopopescu

2.56. Time-Dependent Transport as Critical Phenomenon

$V$. Protopopescu, W. Klein, and T. Keyes

2.57. The Goldstein-McKean Model Revisited

V. Protopopescu and T. Keyes

2.58. Kinetic Equations with Reflecting Boundary Conditions

C. V. M. van der Mee and V. Protopcopescu. 
2.59. Half-range Solutions of Indefinite Strum-Liouville Problems M. Klaus, C. V. M. van der Mee, and V. Protopopescu .

2.60. Stationary Transport with Partially Reflecting Boundary Conditions

$\boldsymbol{R}$ G. Cole, $\boldsymbol{T}$. Keyes, and $\boldsymbol{V}$. Protopopescu . . . . . . . . . .

2.61. Stationary Transport with Partially Reflecting Boundary Conditions. II.

R. G. Cole, V. Protopopescu, and T. Keyes ............... 64

2.62. Abstract Time-Dependent Transport Equations

$\boldsymbol{R}$ Beals and $\boldsymbol{V}$. Protopopescu .......................... 65

2.63. The Abstract Time-Dependent Transport Problem

V. Protopopescu .

2.64. A Survey of Recent Results and Open Problems in Time-Dependent Transport Theory

V. Protopopescu

Section 3. APPLIED PHYSICS AND FUSION REACTOR ANALYSIS

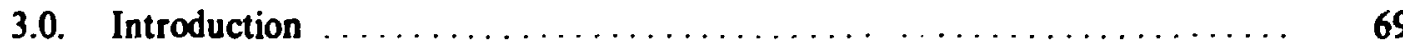

Fusion Reactor Research

3.1. Monte Carlo and Discrete Ordinates Calculations of 14-iMeV Neutzons

Streaming Through a Stainless Steel Duct: Comparison with Experiment I

R. T. Santoro, J. M. Barmes, R. G. Alsmiller, Jr., and

J. D. Drischler.

3.2. Calculations to Evaluate the Use of the Attenuation of D-D Neutrons in Liquid Oxygen as a Means of Measuring Plasma Ion Temperature

R. G. Alsmiller, Jr., R. T. Santoro, J. F. Manneschmidt, and

$J$ M Rnones

3. ?. Multigroup Energy-Angle Distributions for Neutrons from the $\mathrm{T}(d, n)^{4} \mathrm{He}$ Reaction $\left(E_{d}=100-400 \mathrm{keV}\right)$

R. T. Santoro, J. M. Barnes, J. D. Drischler, and R. G.

Alsmiller, Jr.

3.4. ORACT: A 174-Neutron-Group Activation Cross-Section Library for

Fusion and Fission Reactor Design Studies

R. T. Santoro, J. E. White, and J. D. Drischler

3.5. Magnetics Calculations for an ELMO Bumpy Square

R. T. Santoro, N. A. Uckan, and R. J. Schmitt 
3.6. An EBT Keactor Systems Analysis and ( xst Cude: Descriptir a and Iser's Guide (Version 1)

R. T. Santoro, N. A. Uckan, J. M. Bc nes and D. E. Driemeyer .....

3.7. Fusion Reactor Shielding Benchmark I.. Dut: Streaming Experiments ano Analyses

G. T. Chapman, R. T. Santoro, R. G. Als:nit'er, Jr., J. M. Barnes,

J. S. Tang, and P. D. Soran .

High-Energy Accelenato: Shiet ins smi Detector Research

3.8. The High-Energy Transport Crie, HET $\mathrm{C}$

T. A. Gabriel

3.9. Compensation Effects in Hadroa Calorinet -is

T. A. Gabriel, B. L Bishop. '. Brau, A. Di Ciaccio, M. Goodman, ard $\boldsymbol{R}$. Wilson

3.10. Hadron-Lepton Cascade Calcu!ations (1-21, GeV) for a $\mathrm{Pb}$-Al-Lucite Caloiimeter

F. S. Alsmiller, T. A. Gabri.l, and R. C. Alsmiller, Jr. . . . . . . .

3.11. Montc Carlo Studies of Uranium Calorimetry

J. Erau, T. A. Gabriel, B. I Bishop, a"d J. Hargis

3.12. A Monte Carlo Simulation of the Response of a Hadronic Calorimeter to Protons of Momentum 3.5 to $200 \mathrm{GeV} / \mathrm{c}$

A. I. Mincer, T. A. Gabriel, B. L. Bishop, H. T. Freudenreich,

J. A. Goodman, S. C. Tonwar, G. B. Yodh, D. Berley, and

R.W. Ellsworth

3.13. Measurements of the Response of a Hadronic Calorimeter to Beams of Pions and Protons of 1 to $10 \mathrm{GeV}$ Momenia

A. I. Mincer, H. T. Freudenteich, J. A. Goodman, S. C. Tonwar,

G. B. Yodh, D. Berley, R W. Ellsworth, T. A. Gabriel, and

B. L. Bishop

3.14. An Introduction to Combinatorial Geometry

T. A. Gabriel and M. B. Emmett

\section{Fanergy Economics Modeline and Analysis}

3.15. A Model of the World Oil Market with an OPEC Cartel

R. G. Alsmiller. Jr., J. E. Horwedel, R A. Marshalla, D. M. Nesbitt, and S. M. Haas 
Section 4. MATHEMATICAL MODELING AND INTELLIGENT CONTROL

4.0. Introduction

Artificial Intelligence, Robotics

and Adranced Comprater Systens Research

4.1. Artificial Intelligence and Cencurrent Computation for Robotic

Applications

C. R. Weisbin, J. Barhen, G. de Saussure, W. R. Hamel, C. Jorgensen,

J. L. Lucius, E. B. Oblow, and T. Swift

4.2. Center for Engineering Systems Advanced Research Program

C. R. Weisbin and K. McKeehan

4.3. Minimal Cut-Set Methodology for Artificial Intelligence Applications

C. R. Weisbin, G. de Saussure, J. Barhen, E. K. Oblow, and

J. C. White

4.4. Automated Planning for Intelligent Machines in Energy-Related

Applications

C. R. Weisbin, G. de Saussure, and J. Barhen

4.5. Real-Time Planning by an Intelligent Robot

J. Barhen, $C$. $R$ Weisbin, and G. de Saussure.

4.6. Machine Iatelligence for Robotics Applications

C. $R$ Weisbin, J. Barhen, G. de Saussure, W. R Hamel,

C. Jorgensen, E. M. Oblow, and R. E. Ricks

4.7. HERMIES-I: A Mobile Robot for Navigation and Manipulation Experiments

C. R. Weisbin, J. Barhen, G. de Saussure, W. R. Hamel, C. Jorgensen,

J. L. Lucius, E. M. Oblow, and T. Swift

4.8. Hypercube Concurrent Computation and Virtual Time for Robotic Application

J. Barhen

4.9. Robot Inverse Dynamics on a Concurrent Computation Ensemble

J. Barhen

4.10. Control of a Single Link, Two-Degree-of-Freedom Manipulator with Joint Compliance and Actuator Dynamics

M. G. Forrest-Barlach, S. M. Babcock, H. Singh, and M. J. Rabins....

4.11. A Hybrid Uncertainty Theory

E. M. Oblow 


\section{Derekpment and Application of LWR Pressure Vesel Surreillance Dosimetry Amlysis Methods}

4.12. Activity and Fluence Calculations for the Startup and Two-Year Irradiation Experiments Performed at the Poolside Facility

R. E. Maerker and B. A. Worley

4.13. Calculated Spectral Fluences and Dosimeter Activities for the Metallurgical Blind Test Irradiations at the ORR-PSF

R. E. Moerker and B. A. Worley ... . . . . . . . . . . . . . .

4.14. Neutron Calculations of the Two-Year Irradiation Experiment at the ORR-PSF and Comparison with Measurements

R. E. Maerker and B. A. Worley

4.15. Gamma-Ray Characterization of the Two-Year Irradiation Experiment

Performed at the Poolside Facility

R. E. Maerker.

4.16. Validation of Neutron Transport Calculations in Benchmark Facilities for Improved Damage Fluence Predictions

M. L. Williams, F. W. Stallmann, R. F. Mcerker, and

F. B. K. Kam . . . . . . . . . . . . . . . . . . . . . . .

4.17. Theory of a New Unfolding Procedure in PWR Pressure Vessel

Dosimetry and Development of an Associated Benchmark Database

R. E. Maerker, B. L. Broadhead, and J. J. Wagschal

4.18. Revision and Expansion of the Data Base in the LEPRICON Dosimetry

Methodology

R. E. Maerker, M. L. Williams, B. L. Broadhead, J. J. Wagschal, and $C . Y . F u$

4.19. The ELXSIR Cross-Section Library for LWR Pressure Vessel

Irradiation Studies: Part of the LEPRICON Computer Code System

R. E. Maerker, W. E. Ford III, C. C. Webster, and

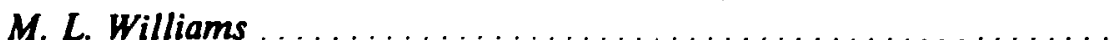

4.20. Unfolded ANO-1 Flixes Using the LEPRICON Methodology

J. J. Wagschal, R. E. Maerker, B. L. Broadhead, and

M. L. Williams

4.21. LEPRICON - A Systematic Approach to LWR Pressure Vessel

Dosimetry

O. Ozer, R. E. Maerker, M. L. Williams, B. L. Broadhead,

J. J. Wagschal, and C. Y. Fu

4.22. Combining Integral and Differential Dosimetry Data in an Unfolding Procedure with Application to the Arkansas Nuclear One-Unit 1 Reactor

R. E. Maerker, B. L. Broadhead, C. Y. Fu, J. J. Wagschal,

J. Williams, and M. L. Williams 
xxiii

4.23. Application of LEPRICON Methodology to the Unfolding of Neutron Fluxes in the ANO-1 Reactor

R. E. Maerker, B. L. Broadhead, and M. L. Williams ........... 94

4.24. Application of the LEPRICON Unfolding Procedure to the Arkansas Nuclear One-Unit 1 Reactor

R. E. Maerker, B. L. Broadhead, B. A. Worley, M. L. Williams,

and J. J. Wagschai .

4.25. Recent Progress and Developments in LWR-PV Calculational Methodology

R. E. Maerker, B. L. Broadhead, and M. L. Williams . . . . . . . 95

4.26. Sensitivities of the Flux Spectrum in the Cavity of a PWR to Variations in the Core Source Distribution

B. L. Broadhead and R. E. Maerker . .................. 95

4.27. Correlations Between Calculated Surveillance Dosimeter Activities and Pressure Vessel Fluxes in the Arkansas Nuclear One-Unit 1 Reactor

$\boldsymbol{R}$. E. Maerker and B. L. Broadhead ......................

\section{Reactor Physics Methods}

4.28. Analysis of Thermal Reactor Benchmarks with Design Codes Based on ENDF/B-V Data

M. L. Williams, R. Q. Wright, B. A. Worley, O. Ozer, and

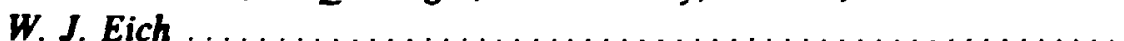

4.29. Improvements in Calculating Neutron Trensmission Probabilities in UnitCell Interface-Current Codes

B. A. Worley

\section{Performance Asacasment of Waste Isolation Systems}

4.30. Radionuclide Migration Pathways Analysis for the Oak Ridge Central Waste Disposal Facility on the West Chestnut Ridge Site

F. G. Pin, J. P Witherspoon, D. W. Lee, J. B. Cannon, and

R. H. Ketelle

4.31. Application of Pathways Analyses for Site Performance Predictira for the Gas Centrifuge Enrichment Plant and Oak Ridge Centra! Waste Disposal Facility

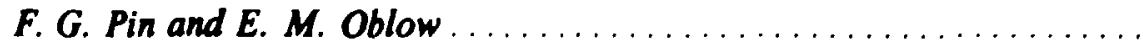

4.32. Pathways Analyses and Their Role in the Decision Making Process for Selection of Low-Level Waste Disposal Sites

F. G. Pin and E. M. Oblow . 
xxiv

4.33. Automated Sensitivity Analysis of the Radionuclide Migration Code UCB.NE-10.2

F. G. Pin, B. A. Worley, E. M. Oblow, R. Q. Wright, and

W. V. Harper.

4.34. GRESS - Gradient-Enhanced Software System - Version D User's Guide

E. M. Oblow

\section{Anlysis of $\mathrm{CO}_{2}$ Impact on Cimate}

4.35. Carbon Dioxide Buildup and Climate: Potential Impacts

M. C. G. Hall

4.36. Estimating the Reliability of Climate Model Projections - Steps

Toward a Solution

M. C. G. Hall

4.37. Feedback in the Climate: Accuracy of First-Order Estimates

M. C. G. Hall

4.38. Efficient Estimation of Feedback Effects with Application to Climatic Models

D. G. Cacuci and M. C. G. Hall .

\section{Section 5. REL:ABLITY AND HUMAN FACTORS RESEARCH}

5.0. Introduction

\section{System Reliability}

5.1. CREDO: The Centralized Reliability Data Organization - A Data Base and Data Analysis for Advanced Reactors

H. E. Knee, P. M. Haas, S. L. Painter, and M. D. Anderson . . . . . . 107

5.2. Human Factors Review for Nuclear Power Plant Severe Accident

Sequence Analysis

P. A. Krois and P. M. Haas

5.3. Human Factors Review for Severe Accident Sequence Analysis (SASA)

P. A. Krois, P. M. Haas, J. J. Manning, and C. $R$ Bovell

5.4. A Review of the Treat Upgrade Reactor Scram System Reliability Analysis

D. F. Montague, J. B. Fussell, P. A. Krois, T. C. Morelock,

H. E. Knee, J. J. Manning, P. M. Haas, and K. W. West 
5.5. CREDO Data Analysis: A Comparison of Sodium and Water Valve Reliabilities

S. L. Painter, M. D. Andersom, and H. E. Knee ........... 108

\section{Heman Performance Mensurement}

5.6. The Effocts of Supervisor Experience and Assistance of a Shift Technical Advisor (STA) on Crew Performance in Control Room Simulators

A. N. Beare, M. D. Donovan, D. L. Lassiter, and L H. Gray ........

5.7. Simulator Experiments: Effects of Experience of Senior Reactor Operators and of Presence of a Shift Technical Advisor on Performance in a Boiling Water Reactor Control Room

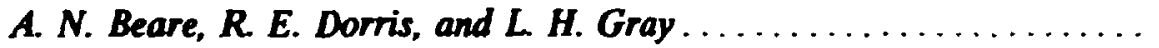

5.8. Simulator Experiments: Effects of NPP Operator Experience on Performance

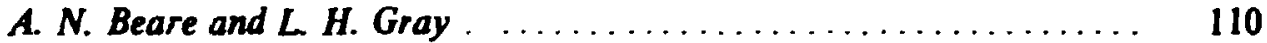

5.9. Control Room Operator Performance Research

L. H. Gray, P. M. Haas, J. J. Manning, and J. C. Lowry . . . . . . .

5.10. Nuclear Power Plant Control Room Operators Performance Research

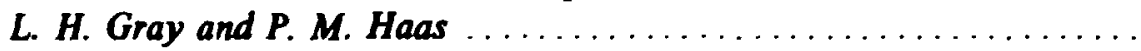

5.11. Collection and Analysis of Training Simulator Data

P. A. Krois and P. M. Haas

Personed Qualifications and Training

5.12. A Classroom Training Revision Methodology

R. J. Carter .

5.13. Nuclear Power Flant Training Simulator Fidelity Assessment

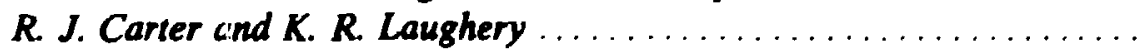

5.14. A Methodology for the Evaluation of Nuclear Power Plant Simulation Facilities

R. J. Carter and K. R. Laughery

5.15. Training Related Research and Development Conducted at Oak Ridge National Laboatory for the U.S. Nuclear Regulatory Commission

P. M. Haas

5.16. Nuclear Power Plant Simulation Facility Evaluation Methodology

P. M. Haas, K. J. Carter, and K. R. Laughery 
5.17. Methads for Evaluation of Nuclear Power Plant Personnel Training and Entry Leve! Oualifications

P. M. Haas, C. C. Jorgensem, D. L. Selby, and J. C. Lowry ........

5.18. Nuclear Power Plant Personnel Qualifications and Training: TSORT An Automated Technique to Assign Tasks to Training Strategies

C. C. Jorgensen .

5.19. Initial Development of an Automated Task Analysis Profiling System

(TAPS)

C. C. Jorgensen

5.20. Nuclear Power Plant Personnel Qualifications and Training: TAPS -

The Task Analysis Profiling System

C. C. Jorgensen.

5.21. Development of Methods for Nuclear Power Plant Personnel

Qualifications and Training

C. C. Jorgensen and R. J. Carter

5.22. A Select Review of the Recent (1979-1983) Behavioral Research

Literature on Training Simulators

K. $R$ Laughery

\section{Human Reliability Assessment}

5.23. The Maintenance Personnel Performance Simulation (MAPPS) Model:

A Human Reliability Analysis Tool

H. E. Knee

5.24. Maintenance Personnel Performance Simulation (MAPPS) Modei:

Overview and Evaluation Effores

H. E. Knee, P. M. Haas, T. G. Ryan, A. I. Siegel, W. D. Bartter, and J. J. Wolf .

5.25. Maintenance Personnel Performance Simulation (MAPPS) Model:

Description of Model Content, Structure, and Sensitivity Testing

A. I. Siegel, W. D. Bartter, J. J. Wolf, and H. E. Knee . . . . . . . .

5.26. The Maintenance Personnel Performance Simulation (MAPPS) Model

A. I. Siegel, W. D. Bartter, J. J. Wolf, H. E. Knee, and

P. M. Haas

5.27. Maintenance Personnel Performance Simulation (MAPPS) Model:

Summary Description

A. I. Siegel, W. D. Bartter, J. J. Wolf, H. E. Knee, and P. M. Haas. . . 
xxvii

\section{Hemen Factors Progran Manageneat}

5.28. Human Factors Research at Oak Ridge National Laboratory

P. M. Haas

\section{Section 6. APPLIED RISK AND DECISION ANALYSIS}

6.0. Introfuction

6.1. PRISIM: A PC Program to Display Risk Status Information

D. J. Campbell, B. C. Ellison, J. C. Glym, and G. F. Flanagan .......

6.2. A Methodology for Comparing the Health Effects of Electricity

Generation from Uranium and Coal Fuels

W. R. Rhyne and A. A. Ei-Bassioni

6.3. Modeling the Detection Rates of Fires in Nuclear Power Plasts

$N$. Siu and G. Apostolakis

6.4. Integration of PTS Studies to Calculate Through-the-Wall Crack

Probabilities

D. L. Selby and J. D. White

6.5. Prioritization of a Large Number of R\&D Programs

G. Flanagan and V. R. R. Uppuluri

\section{Section 7. INFORMATION ANALYSIS AND DATA MANAGEMENT}

7.0. Introduction

7.1. The Radiation Shielding Information Center: A Source of Computer Codes and Data for Dosimetry Applications

D. K. Trubey, B. F. Maskewitz, and R. W. Roussin .

7.2. The Radiation Shielding Information Center's Foreign Technology Assessment - An Important National Asset

B. F. Maskewilz

7.3. Abstracts of Digital Computer Code Packages Assembled by the Radiation Shielding Information Center

B. J. Carter and B. F. Maskewitz

7.4. Overview of the Role of Data for Ensrgy Systems

B. F. Maskewitz and J. A. Watts

7.5. VITAMIN-E $174 n, 38 g$ General-Purpose Cross-Section Library

R. W. Roussin, J. E. White, and J. L. Bartley 
xxviii

7.6. VITAMIN-E 174-Neutron, 38-Gamma Cross-Section Library for Fusion Neutrcnics Calculations

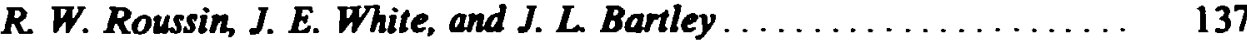

7.7. Beginner's Guide to Blocked Records on Magnetic Tape

B. L. Kirk

7.8. Systems Guide to MCNP (Monte Carlo Neutron and Photon Transport Code)

B. L. Kirk and J. T. West

7.9. NRC Technical Data Management Center (TDMC)

J. E. White, R W. Roussin, and B. F. Maskewitz

7.10. User's Guide for the NRC Staff Version of CRAC2

J. E. White and R. W. Roussin

7.11. Effect of Fluorescence, Bremsstrahlung, and Annihilation Radiation on the Spectra and Energy Deposition of Gamma Rays in Bulk Media

K. V. Subbaiah, A. Natarajan, D. V. Gopinath, and D. K. Trubey

7.12. Carbon Dioxide Information Center (CDIC): A Progress Report for the Period January 1982 - September 1983

B. F. Maskewitz, J. A. Watts, C. R. Weisbin, L. J. Allison,

B. F. Jacobs, anc H. A. Pfuderer

\section{Section 8. MATHEMATICAL SCIENCES}

8.0. Introduction

\section{Applied Mathrmatics}

8.1. A New Numerical Method for Phase Change Problems

$V$. Alexiades

8.2. Alloy Solidification with Constitutional Supercooling

$V$. Alexiades

8.3. Remarks and Quesiions About Hyperbolic Stefan Problems

V. Alexiades

8.4. The ORNL Binary Alloy Solidification Model

$V$. Alexiades

8.5. Minimizers of the Free Energy in the Thermodynamic Theory of

Fluid Interfaces

$V$. Alexiades 
8.6. A General Model of Binary Alloy Solidification

V. Alexiades, D. G. Wilson, and A. D. Solomon

8.7. On the Thermodynamic Theory of Fluid Interfaces: Infinite

Intervals Equilibrium Solutions, and Minimizers

$V$. Alexiades and E. C. Aifantis .

8.8. Numerical Simulation of a $\mathrm{HgCdTe}$ Solidification Process

V. Alexiades, G. A. Geist, and A. D. Solomon . . . . . . . . .

8.?. Macroscopic Global Modeling of Binary Alloy Solidification Processes

$V$. Alexiades, D. G. Wilsom, and A. D. Solomon ...............

8.10. PCMSOL1 - A Direct Gain Solar Simulation Code with Phase

Change Materials

A. Geist, A. Solomon, J. Tomlinson, and C. Serbin .............

8.11. Self-Consistent Approximations for Random Alloys

L. J. Gray . . . . . . . . . . . . . . . . . . . . . . . . .

8.12. A Fractal Model for Charge Diffusion Across a Rough Interface

L. J. Gray, S. H. Liu, and T. Kaplan

8.13. Simulation of the Electrochemical Machining Process

L. J. G. zy, C. A. Serbin and W. C. Dietrich

8.14. Self-Consistent Approximation for Muffin-Tin Models of Random

Substitutional Alloys with Environmental Disorder

T. Kaplan and L. J. Gray

8.15. Role of Mathematics and Statistics Research Section of Oak Ridge

National Laboratory

C. A. Serbin.

8.16. The Mathematics of Artificial Intelligence

A. D. Solomon

8.17. Approximation Methods for Phase Change Problems

A. D. Solomon

8.18. Mathematical Methods in Latent Heat Storage

A. D. Solomon

8.19. Pitfalls of Computer Simulation

A. D. Solomon

8.20. Numerical Methods for Solving Stefan-Type Problems

A. D. Solomon 
8.21. What Is Mathematics to Us?

A. D. Solomon

8.22. The Initial Speed of an Emerging Phase Change Front

A. D. Solomon, V. Alexiades, ard D. G. Wilson

8.23. The Initial Velocity of the Emerging Free Boundary in a Two-Phase

Stefan Problem with Imposed Flux

A. D. Solomon, V. Alexiades, and D. G. Wilson

8.24. Numerical Simulation of a Binary Alloy Solidification Process

A. D. Solomon, V. Alexiades, and D. G. Wilson

8.25. Moving Boundary Problems in Phase Change Models: Current Research Questions

A. D. Solomon, V. Alexiades, and D. G. Wilson

8.26. The Formulation of a Hyperbolic Stefan Problem

A. D. Solomon, V. Alexiades, D. G. Wilson, and J. Drake

8.27. Heat Transfer Considerations in Passive Solar with Energy Storage

A. D. Solomon, G. A. Geist, and C. A. Serbin ... . . . . . . . .

8.28. Heat Transfer and Phase Change Simulation in PCMSOL-I, A Model

of a Direct Gain Passive Solar Structure with Latent Heat Storage

A. D. Solomon, J. J. Tomlinson, G. A. Geist, and C. A. Serbin

8.29. The Quasi-Stationary Approximation for the Stefan Problem with a Convective Boundary Condition

A. D. Solomon, D. G. Wilsom and V. Alexiades

8.30. A Device Which Allows Special Event Detection in Continuous Simulation Languages Without Discrete Blocks

S. Thompson

8.31. Event Detection in Continuous Simulation

S. Thompson and P. G. Tuttle

8.32. Time Dependent Linear Transport III - Convergence of the Discrete Ordinates Method

D. G. Wilson

8.33. Vectorization of Multidimensional Finite Difference Schemes with Applica: ion to Stefan-Type Problems

D. G. Wilson

8.34. Mathematical Modeling - Fun and Games with Applications

D. G. Wilson 
8.35. Modeling Binary Alloy Solidification

D. G. Wilson . . . . . . . . . . . . . . . . . . . . . . .

8.36. Computer Modeling of Solidification Processes

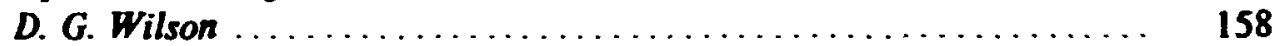

8.37. The 1984 Model of Binary Alloy Solidification

D. G. Wilson, V. Alexiades, and A. D. Solomon ............ 158

8.38. A Model of Binary Alloy Solidification

D. G. Wilson, A. D. Solomon, and V. Alexiades

159

\section{Compenter Science}

8.39. An Algorithm to Compute a Sparse Basis of the Null Space

M. W. Berry, M. T. Heath, I. Kaneko, M. Lawo, R. J. Plemmons,

and R. C. Ward

159

8.40. Orthogonal Schemes for Structural Optimization

M. W. Berry, M. T. Heath, R. J. Plemmons, and R. C. Ward ......

8.41. Progress Report on Japanese Computer Projects

K. O. Bowman

160

8.42. The Hypercube Architecture and Some Aspects of Data Flow for Parallel Computation of Missized Matrix Problems

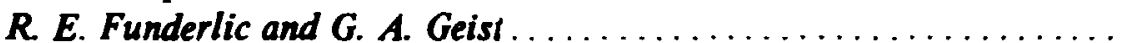

8.43. Torus Data Flow for Parallel Computation of Missized Matrix Problems

$\boldsymbol{R}$ E. Funderlic and $G$. A. Geist

8.44. Sensitivity of the Stationary Distribution Vector for an Ergodic Markov Chain

R. E. Funderlic and C. D. Meyer, Jr.

8.45. A Combined Direct-Iterative Method for Certain M-Matrix Linear Systems

R. E. Funderlic and R. J. Plemmons.

8.46. Updating LU Factorizations for Computing Stationary Distributions

R. E. Funderlic and R. J. Plemmons.

8.47. FORTRAN Programming on an IBM PC

P. W. Gaffney

8.48. Some Aspects of a Data Flow Algorithm for Cholesky Decomposition

G. A. Geist, R. E. Funderlic, and J. Meyering . 
xxxii

8.49. Parallel Cholesky Factorization on a Hypercube Multiprocessor

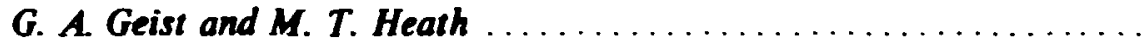

8.50. Parallel Cholesky Factorization on a Multiprocessor

A. George, M. T. Heath, and J. Liu

8.51. Solution of Sparse Underdetermined Systems of Linear Equations

A. George, M. T. Heath, and E. $\mathrm{Ng}$

8.52. Householder Reflections Versus Givens Rotations in Sparse

Orthogonal Decomposition

A. George and J. Liu

8.53. Orthogonal Reduction of Sparse Matrices to Upper Triangular Form Using Householder Transformations

A. George and $\mathrm{E}$. $\mathrm{Ng}$

8.54. Symbolic Factorization for Sparse Gaussian Elimination with Partial Pivoting

A. George and E. $\mathrm{Ng}$

8.55. An Implementation of Gaussian Elimination with Partial Pivoting for Sparse Systems

A. George and $\mathrm{E} . \mathrm{Ng}$

8.56. Parallel Cholesky Factorization in Message-Passing Multiprocessor Environments

M. T. Heath

8.57. Numerical Methods for Large Sparse Linear Least Squares Problems M. T. Heath

8.58. Some Sparse Matrix Problems in Structural Analysis

M. T. Heath

8.59. Sparse Matrix Computations

M. T. Heath

8.60. Computing the Singular Value Decomposition of a Product of Two Matrices

M. T. Heath, A. J. Laub, C. C. Paige, and R. C. Ward.

8.61. A Pipelined Givens" "ethod for Computing the QR Factorization of a Sparse Matrix

M. T. Heath and D. C. Sorensen

8.62. Sparse Orthogonal Schemes for Structural Optimization Using the Force Method

M. T. Heath, R. C. Ward, and R. J. Plemmons 
xxxiii

8.63. On General Row Merging Schemes for Sparse Givens Transformations

J. W. H. Liu

8.i4. Parallel Block Jacobi Eigenvalue Algorithms Using Systolic Arrays

D. S. Scott, M. T. Healh, and R. C. Ward.

\section{Statiotics}

8.65. Misclassification Probabilities for Second-Order Discriminant Functions

Used to Classify Bivariate Normal Populations

C. K. Bayne, J. J. Beauchamp, and V. E. Kane . . . . . . . . . 169

8.66. Application of the Power-Shift Transformation

J. J. Beauchamp and V. E. Kane . . . . . . . . . . . . . . 169

8.67. Subsampling Program for the Estimation of Fish Impingement

J. J. Beauchamp and K. D. Kumar

8.68. Extended Moment Series and the Parameters of the Neg-tive Bizomial Distribution

K. O. Bowman

8.69. Computation of the Polygan!ma Functions

K. O. Bowman

8.70. Approximations to Distributions

8.71. Bounds for the Expectation of the kth Root of a Random Variable

K. G. Bowman, H. K. Lam, and L. R. Shenton

8.72. Bounds for Certain Integrals

K. O. Bowman, H. K. Lam, and L. R. Shenton

8.73. Bounds for Integrals Using Segments

K. O. Bowman, H.K. Lam, and L. R. Shenton

8.74. The Distribution of Moment Estimators for Parameters of the Generalized Poisson Distribution

K. O. Bowman and $L$. R. Shenton

8.75. The Distribution of a Moment Estimator for a Parameter of the Generalized Poisson Distribution

K. O. Bowman and L. R. Shenton

8.76. The Dark Side of Asymptotics and Moment Series

K. O. Bowman and L. R. Shenton 
xxiv

8.77. Study of Age Dependent Half-Life of Iodine in Man: A ReinforcementDepletion Um Model

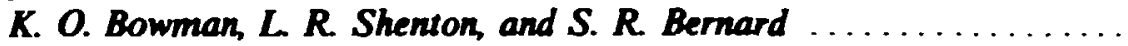

8.79. A Reinforcement-Depletion Urn Model: A Contiguity Model

K. O. Bowman, L. R. Shenton, and S. R. Bemard

8.79. A Poisson Sum Up to the Mean and a Ramanujan Problem

K. O. Bowman, L. R. Shenton, and G. Szekeres

8.80. Fuzzy Hierarchical Analysis

J. J. Buckley and V. R. R. Uppuluri .

8.81. Quantitative Aspects of Metal Ion Content and Toxicity in Drosophila

N. T. Christie, D. G. Gosslee, L. C. Bate, and K. B. Jacobson

8.82. Regression Methods for Binomial and Poisson Distributed Data

E. L. Frome . . . . . . . . . . . . . . . . . . . . . . .

8.83. Response to Nelder's Reaction on Poisson Rate Analysis

E. L. Frome

8.84. The Use of Poisson Regression Models in Estimating Incidence Rates and Ratios

E. L Frome and H. Checkoway

8.85. Application of Poisson Regression to the Analysis of Childhood Leukemia Deaths in Utah .

E. L. Frome and D. L. Cragle

8.86. A Macro for Fitting Nonlinear Models to Poisson Distributed Data

E. L. Frome and R. McLain

8.87. Response of Mouse Spermatogonial Stem Cells to X-Ray Induction of Heritable Reciprocal Translocations

W. M. Generoso, K. T. Cain, N. L. A. Cacheiro, C. V. Cornett, and D. G. Gosslee.

8.88. Difference in the Response of Two Hybrid Stocks of Mice to X-Ray Induction of Chromosome Aberrations in Spermatogonial Stem Cells W. M. Generoso, K. T. Cain, C. V. Cormett, N. L. A. Cacheiro, L. A. Hughes, P. W. Braden, and D. G. Gosslee

8.89. Estimation of the Minimum Latent Period for Radiogenic Osteosarcomas in Man

P. G. Groer and V. R. R. Uppuluri 
8.90. Statistical Evaluations in the Carcinogenesis Bioassay of Petroleum Hydrocarbons

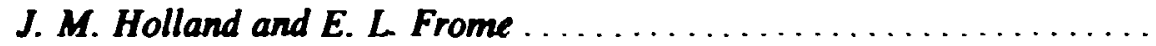

8.91. A Stochastic Model for Estimation of Environmental Density

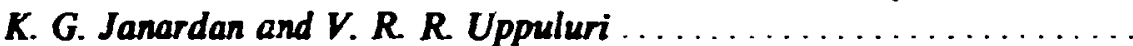

8.92. Inhalation Toxicology of Diesel Fuel Obscurant Aerosol in Sprague-

Dawley Rats

S. Lock, W. Dalbey, R. L. Schmoyer, and R Griesemer .. ........

8.93. First Digits of Exponentially Distributed Random Variables

C. E. McHenry, S. A. Patil, and V. R. R. Uppuluri

8.94. Application of Maximum Likelihood Paired Comparison $\mathbf{R}$ anking to

Estimation of a Linear Dominance Hierarchy in Animal Societies

C. A. McMahan and M. D. Morris

8.95. Computer Construction of Supersaturated Screening Designs

T. J. Mitchell

180

8.96. Minimum Number of Runs for Two-Level Factorial Search Designs

M. D. Morris.

8.97. An Interesting Property of the Sample Mean Under a First-Order Autoregressive Model

M. D. Morris and S. F. Ebey

8.98. On the Precision of the Average of Correlated Measurements

M. D. Morris and S. F. Ebey

8.99. ANOVA Model Fitting via Sparse Matrix Computations

G. Ostrouchov

8.100. Large Sparse Least Squares Computations

G. Ostrouchov

8.101. Symbolic Givens Reduction in Large Sparse Least Squares Problems

G. Ostrouchov

8.102. Effect of Multiple Sequential Thymus Grafting on Immume Competence and Mean Life Span

E. H. Perkins, P. L. Glover, and D. G. Gosslee

8.103. Software Systems for Statistical Process Control and Simulated-Process Optimization

D. C. Schlotzhauer

8.104. Sigmoidally Constrained Maximum Likelihood Estimation in Quantal Bioassay

R. L. Schmoyer 


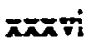

8.105. Everyday Application of the Cell Means Model

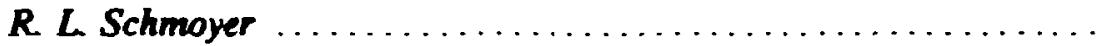

8.106. A Distribution-Free Analysis for Accelerated Testing at Several Levels of a Single Stress

$R$ L Schmoyer

183

8.107. A Nonparametric Analysis for Steep Dose-Response Bioassays

$R$ L Schmoyer, J. J. Beauchamp, and J. McCarthy .

8.108. Distributions Arising from Reinforcement-Depletion Um Model

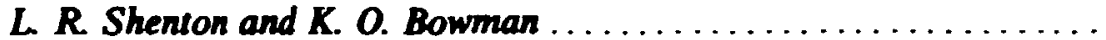

8.109. On the Distribution of the Time Required to Remove White Balls

from an Urn

M. Sobel, S. R Bernard, and V. R R Uppuluri

8.110. Four-Um Catenary Model for Excretion

M. Sobel, S. R Bernard, $V . R$ R Uppuluri, and C. W. Nestor, Jr. .

8.111. Limiting Values for the RBE of Fission Neutrons at Low Doses for

Life Shortening in Mice

J. B. Storer and T. J. Mitchell

8.112. An Empirical Study on the Stabilities of Difference Estimators

$H$. Tsao and T. Wright

8.113. An Empirical Study on Maximizing (or Minimizing) the Number of Retentions in Unequal Probability Sampling Without Replacement: Two Units Per Stratum

H. Tsao and T. Wright

8.114. Nonparametric Estimation of the Distribution of Time to Onset for Specific Diseases in Survival/Sacrifice Experiments

B. W. Turnbull and T. J. Mitchell

8.115. Methods in Risk Management - A wojricing Paper

V. R. R. Uppuluri

8.116. The Distribution of the First $j$ Digits of Beta Related Random Variables

V. R. R. Uppuluri and S. A. Patil.

8.117. Statistical Methods and the Improvement of Data Quality

T. Wright

8.118. Some Useful Notes on Simple Random Sampling

$T$ Wright and H. Tsao 
$x \times x$ vii

8.119. On an Optimal Solution for Maximizing the Probability of Retention in PPS Sampling

T. Wright and H. J. Tsao

8.120. Drinking Water Inorganics and Cardiovascular Disease: A Case-Control Study Among Wisconsin Farmers

E. A. Zeighami, M. D. Morris, E. E. Calle, P. S. McSweeny, and

B. A. Schuknecht ..............................

188

\section{APPENDICDS}

Scientific and Professional Activities $\ldots \ldots \ldots \ldots \ldots \ldots \ldots \ldots \ldots \ldots \ldots \ldots$

Engineering Physics and Mathematies Division Seminars at ORNL . . . . . 217

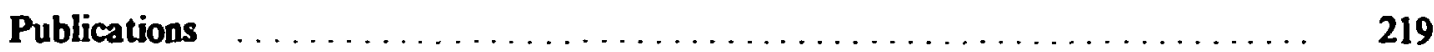

Papers Presented at Scientific Meetings and Seminars . . . . . . . . . . 233

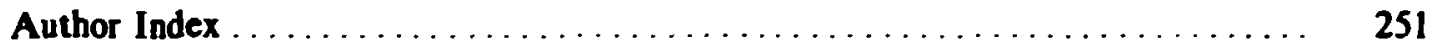

Division Organization Chart $\ldots \ldots \ldots \ldots \ldots \ldots \ldots \ldots \ldots \ldots \ldots \ldots \ldots \ldots \ldots$ 
Section 1

NUCLEAR DATA MEASUREMENTS AND EVALUATION 


\title{
1.0. INTRODUCTION
}

\author{
R. W. Peele
}

: The recent wrenching national changes in the U. S. Department of Energy's reactor development programs have led to the transfer of responsibility for the nuclear data research previously sponsored as part the Office of Nuctear Energy's Fast Reactor Program to the Office of Energy Research's Basic Energy Sciences (BES) Program. The tranefer was not accompanied by any increase in funding, so the research program at the Oat Ridge Electron Linear Accelerator (ORELA) has suffered an overall loos of about one-fourth of its funding in a two-year time apan. (Other laboratories have suffered larger relative cuts.) Now, almost all of the nuclear dats measurement and evaluation program is sponsored by BES. While the tranafer of engincers and scientists out of the ORELA program was a severe setbact that is decreasing our output, good fortune permitted the transition to be performed with far less confusion and loas of data than had been feared. Now that the BES program is the overwhelmingly dominant sponeor of OREIA work, one can expect a shift in research priorities to give less emphasis on fast reactor data needs, even though the most important of those needs are so general as to invite new research efforts. In fact, completion of the fast reactor research that was under way at ORELA will be required for the success of any of the reactor options still under consideration for development.

The ORELA itself has been operated to exemplary standards in the face of engineering staff reductions. At the beginring of the reporting period, the prebuncher equipment, derigned to compress a 15-ns-wide injec or pulse to 4 ns prior to acceleration, was removed. Mout of the diffculties experienced in testing, this device are understood and could be compenated for by: redeciza, but contradictory data on the acceptance efficiency for the beam that enters the first RF acceleration stage must be resolved prior to taking this step. Anotber development project now under way is to deflect after-pulse electrons away from the neutron-producing torget, which will often allow increased output by permitting the eloctron gun to be driven to bigher levels.

We have now completid a decade-long program to provide definitive measurements of the average number of prompe reutrons emitted per fission from each of the major fiscile nuclides as a function of neutron evergy. As a result of cur program, which included measurements that reduced the uncertainty in the number of neutrons emitted per fiscion from the ${ }^{252} \mathrm{Cr}$ spontancous fiecion standard, the uncertainty in the inesion neutron multiplicity averaged over reactor apectra is now believed to be no more than $0.3 \%$, an experimental reault well worth more than a docade of dedicated effort.

Another "victory" in the experimental program has been the publication of the last of a series of measurements of the capture cross sections of a "inide nuclides produced in resctors as tranemutation products. The nuclide ${ }^{243} \mathrm{Am}$ was the lant on: studied and the moet difficult bocause of its intense radioactivity. This program was part of a quite succeaf ul international effort atarted a decade ago to alleviate a serious lack of information neoded for aucaument of spent fuel handling from fast reactors and for analysis of "actinide burning" to reduce the loag-lerm waste hazard from actinides in a cloced fission reactor fuel cycle system. Many fission croses soctions of beavy actinides were also measured at OREL.A in this program. 
A prime problem of fiscion reactor physics has ever been the correct analysis of the effects of neutron absorption in the resonances of ${ }^{238} \mathrm{U}$ (or ${ }^{232} \mathrm{Th}$ in the case of thorium cycle reactors). While the auclear data for ${ }^{231} \mathrm{U}$ are good by usual standards and reflect great research efforts, most integral measurements of reactor parameters that involve ${ }^{234} \mathrm{U}$ still disagree with calculations and indicate that even better resconance data will be required to eliminate the limiting discrepancies that arise from the ${ }^{238} \mathrm{U}$ cross section. One obvious problem has been that the nominal resonance region recognized in the reference ENDF/B cross-section set terminates at 4teV neutron energy, while the measured cross sections show strong resonance fluctuations up to over $10 \mathrm{keV}$. Until recently there also seemed to be discrepancies of unknown origin among the data sets best regarded worldwide. A special international task group appointed by the Nuclear Energy Agency Nuclear Data Committee set out to resolve these discrepancies and indeed demonstrated that the data sets themselves were consistent though of uneven quality. (The ORELA data were best) Analysis techniques were refined in the process, and that already has led to a resonance analysis of ORELA data to $10 \mathrm{keV}$ that shou': belp solve the major reactor physics problem identified above when it is incorporated into future reference data sets. Perhaps more important in the long run, the proof that the existing data sets were consistent has unleashed the imagination of experimenters who had previously been held back by the discrepancy and the resulting fear that additional work could yield no gain. Now it is apparent how new and improved techniques can lead to even better data and how newly improved analysis methods can then lead at last to a description of neutron resonance reactions with ${ }^{238} \mathrm{U}$ that will be fully adequate for the analysis of future fission reactor systems. The work is not yet started to obtain the new data; present efforts are being concentrated on ${ }^{230} \mathrm{Pu}$, for which past data have also proven to be inadequate.

Great strides continue toward advanced ability to reduce and analyze ORELA data in a manner that preserves the information content and thereby properly projects the uncertainties in all results. The SAMMY and ALEX computer programs mentioned in some of the abstracts below put into practice the theoretical concepts of data reduction that were developed earlier. These programs are currently being employed, the comprehensive analyses of the resonance structures of ${ }^{5 /} \mathrm{Ni}$ and ${ }^{239} \mathrm{Pu}$ being cases in point. We hope to continue to pursue definitive resonance analyses for structural materials and for fuel nuclides.

Since the data records obtained at ORELA may include the contents of hundreds of thousands of time-of-night channels that must be corrected, combined, and analyzed in terms of the nuclear parameters being studied, the provision of powerful data-acquisition, display, and analysis computer systems has been crucial to productivity. Much of the initial ORELA equipment has become obsolete, though it functions well enough for the originally defined tasks. Presently a VAX 11/785 is being installed to replace the original PDP-10 system, and the complex data-analysis techniques are being further facilitated by the addition of a matrix-procensing ("Maxboard") system on the FPS-164 presently employed only by the Nuclear Theory Group in the Physics Division. These new linked machines will provide the capability to perform the increasingly complex data reduction and analysis tasks with very much improved efficiency. So far it appears that computer changeover problems can be well accommodated. Significant iniprovements to the data-acquisition system are also under way, but wholesale replacement of that system is not presently planned.

The data evaluation function provides complete sets of neutron cross sections for materials of importance to technology. In some cases, this process involves the systematic combination of experimental data, which we do in helping to provile standard sets of evaluated cross sections. At neutron energies above a few MeV, which are important to fusion power, the inability to study all important reactions by experiment requires that the evaluators place considerable reliance on nuclear moiv! codes. Invention and validaties of code aystems such as our ING referred to below therefore play a maj:- role in neutron crose-section evaluation. 
The 61 abstracts published in this section reflect that portion of our work that reached the reporting stage during the 18 months covered by the progress report. Many of the results on total, fission, capture, and other cross sections are noteworthy of themselves or have interesting interpretations. The first subsection contains abstracts of papers that include new experimental results from ORELA; the second subsection covers cross-section evaluations, theoretical evaluation techJiques, and reviews of our work area; and the last subsection contains abstracts of papers on techniques and facilities. The ORELA output is markedly enhanced by the work of cooperating visitors from many institutions who have shared the ORELA facility with us and correspondingly enriched us all. 


\section{Experimental Results}

1.1

\section{HIGH-RESOLUTION STRUCTURAL MATERIAL $(n, x \gamma)$ PRODUCTION CROSS SECTIONS FOR E FROM 0.2 TO $40 \mathrm{MeV} *$}

\section{C. Larson}

(Abstnet of paper presented at the International Conferense on Nuckea: Dete for Basic and Applied Sciences, Santa Fe, NM, May 13-17, 1985)

A program has teen initiated at the Oak Ridge Electron Linear Accelerator (ORELA) to measure yields of individual gamma rays resulting from interactions of $0.2-$ to $40-\mathrm{MeV}$ neutrons with structural materials of interest both for applied needs and basic research. A highresolution gamma-ray detector and twoparameter (neutron time-of-flight vs gamma-ray pulse height) techniques are used to record approximateiy 40 pulse-height $4 \mathrm{~K}$-channel spectra for $0.2 \leqslant E_{\gamma} \leqslant 5.1 \mathrm{MeV}$ corresponding to approximately 40 incident neutron-energy bins covering the $0.2-$ to $40-\mathrm{MeV}$ range. The neutron flight-path length is $22 \mathrm{~m}$, and the well-shielded $\mathrm{Ge}(\mathrm{Li})$ detector is located at an angle of $125^{\circ}$ with respect to the incident beam direction and positioned $40 \mathrm{~cm}$ from the center of the sample under study. The neutron flux required for reduction of the data to absolute cross sections was carefully measured using NE-110 detectors and Monte Carlo calculations were employed to establish the absolute efficiencies of these flux detectors. A small NE-110 detector was used as a monitor detector to determine the number of neutrons striking the sample. The efficiency and resolution of the gamma-ray detector as a function of gamma-ray energy was measured using sources for $0.06 \leqslant E_{\gamma} \leqslant 6.1 \mathrm{MeV}$. The electronic setup for the experiment is straightforward with the gamma flash from the accelerator being analyzed and stored to provide a constant neutron-energy calibration, and digitization of the pulse height near the detector being done to eliminate noise pickup over the $\approx 40 \mathrm{~m}$ of cable to the analysis area. Data are accumulated and stored in a SEL-810 computer.
Data have been acquired for samples of ${ }^{7} \mathrm{Li}$, ${ }^{56,57} \mathrm{Fe},{ }^{-1,58} \mathrm{Ni}$ and ${ }^{-53} \mathrm{Cr}$. Gamma rays from residual nuclei of the $\left(n, n^{\prime} \gamma\right),\left(n, p^{\prime} \gamma\right)$ and $\left(n, \alpha^{\prime} \gamma\right)$ binary reactions are observed. For the even-even nuclei studied, most $(\approx 90 \%)$ of the excited states decay through the firs: $2^{+}$level, so the cross section for the gamma ray corresponding to the decay of this level gives a good estimate of the total inelastic scattering cross section for these nuclei. The excellent resolution of the $\mathrm{Ge}(\mathrm{Li})$ detector allows us to observe other ground-state transitions and further improve the estimate for this cross section.

Of particular interest are the gamma rays which we observe from the residual nuclei following tertiary reactions such as $\left(n, 2 n^{\prime} \gamma\right)$, $\left(n, n^{\prime} \alpha \gamma\right)$ and $\left(n, n^{\prime} p \gamma\right)$. The gamma-ray measurements provide a uniaue way of obtaining information on cross sections for these reactions, since the gamma-ray energies are unique signatures of particular tertiary reactions. With ORELA, we are able to obtain information about cross sections for many of these reactions over a wide incident neutron energy range. Information on tertiary reaction cross sections are important, for example, in fusion reactor design applications for heating, radiation damage, and transport calculations. Our data provide cross sections necessary in the development of nuclear models to calculate cross sections for all tertiary reactions, many of which are difficult, if not impossible, to measure experimentally.

-Research sponsored by U.S. DOE Office of Basic Energy Sciences.

1.2

\section{FAST NEUTRON INELASTIC SCATTERING FROM ${ }^{33} \mathrm{Cr}$ *}
J. K. Dickens
D. C. Larson

[A:atract of Bull. Am. Phys. Soc. 29, 1028 (1984)]

ORELA produced neutrons were used to measure gemma-ray production cross sections due 
to neutron interactions with ${ }^{53} \mathrm{Cr}$ for incident neutron energies $0.2 \leqslant E_{n} \leqslant 30 \mathrm{MeV}$. Data reduction for ${ }^{53} \mathrm{Cr}$ has been completed, and some of the excitation functions have been compared with compound-nucleus calculations. New information on the level structure of ${ }^{53} \mathrm{Cr}$ has been deduced for excitation energies up to $4.3 \mathrm{MeV}$.

*Research sponsored by U.S. DOE Office of Basic Energy Sciences.

\section{3}

MEASUREMENTS OF THE ENERGY
DEPENDENCE OF PROMPT NEUTRON
EMISSION FROM ${ }^{20} \mathrm{U},{ }^{204} \mathrm{U}^{20}{ }^{20}$ AND
${ }^{201}$ Pu FOR $E_{ \pm}=0.005$ TO $10 \mathrm{eV}$
RELATIVE TO EMISSION FROM
SPONIANEOUS FISSION OF ${ }^{252} \mathrm{Cr}$

\section{R. Gwh R. R. Spencer} R. W. Indet

[Abatract of Nuel. Sci. Eng. 87, 381 (1984)]

A series of experiments have been performed to measure the dependence on the incident neutron energy of the average number of prompt neutrons emitted per fission from ${ }^{233} \mathrm{U},{ }^{235} \mathrm{U}$, ${ }^{239} \mathrm{Pu}$, and ${ }^{241} \mathrm{Pu}$ relative to the average number of prompt neutrons emitted in spontaneous fission of ${ }^{252} \mathrm{Cf}$. The incident neutron energy range was 0.005 to $10 \mathrm{eV}$. A white neutron source was generated by the Oak Ridge Electron Linear Accelerator, and the energies of the neutrons incident on the fissile samples were determined by time-of-flight techniques. In each experiment the samples, including the ${ }^{252} \mathrm{Cf}$ standard, were contained in different sections of a fission chamber that was surrounded by a large volume $\left(0.91 \mathrm{~m}^{3}\right)$ of liquid scintillator loaded with gadolinium. The fission chamber detected fission events, and the scintillator detected the accompanying prompt neutrons. The resulting data were analyzed to yield: $R_{p}(E)=$ $\nu_{p}(E)$ [fissile] $/ \nu_{p}\left[{ }^{252} \mathrm{Cf}\right.$ ]. Only for ${ }^{239} \mathrm{Pu}$ was any neutron energy dependence definitely snnfirmed, with $R_{p}(E)$ for ${ }^{239} \mathrm{Pu}$ being lower by $0.7 \%$ in the resonance at $0.3 \mathrm{eV}$ than it was near $0.025 \mathrm{eV}$. For incident energies of 0.02 to $0.05 \mathrm{eV}$, values of
$R_{p}(E)$ were $0.6597 \pm 0.0018$ for ${ }^{233} U, 0.6443 \pm$ 0.0014 for ${ }^{235} \mathrm{U}, 0.7655 \pm 0.0014$ for ${ }^{239} \mathrm{Pu}$, and $0.7820 \pm 0.0018$ for ${ }^{241} \mathrm{Pu}$.

*Research sponsored by U.S. DOE Office of Reactor Research and Technology.

IInstrumentation and Controls Divivion.

1.4

MEASUREMENT OF THE ENERGY DEPENDENCE OF PROMPT NEUTRON EMISSION FROM ${ }^{203}$ U, ${ }^{23} \mathrm{U}$, AND 230 POR $E_{\mathrm{a}}=0.0005$ to $10 \mathrm{MeV}$ RELATIVE TO EMISSION FROM SPONTANEOUS FISSION OF ${ }^{252}$

$$
\begin{aligned}
& \text { R. Gin R. R. Spencert } \\
& \text { R. W. L.jet }
\end{aligned}
$$

(Abatract of paper preaented at the International Conference on Nuclear Datu for Basic and Applied Sciescea, Santa Fa NM, May 13-17, 1985)

A series of experiments have been performed to measure the dependence on the incident neutron energy of the average number of prompt neutrons emitted per fission of ${ }^{233} \mathrm{U},{ }^{235} \mathrm{U}$, and ${ }^{239} \mathrm{Pu}$ relative to the average number of prompt neutrons emitted in spontancous fission of ${ }^{252} \mathrm{C}$. The incident energy range was 0.0005 to 10 MeV. A white neutron source was generated by the Oak Ridge Electron Linear Accelerator and the energies of the neutrons incident on the fissile samples were determined by time-of-flight techniques. The fissile sample and the ${ }^{252} \mathrm{Cf}$ standard were continued in a fission chamber surrounded by a large volume $\left(0.91 \mathrm{~m}^{3}\right)$ liquid scintillator loaded with gadolinium. Fissions were detected by the fission chamber and the neutrons were detected by the scintillator. The results for ${ }^{239} \mathrm{Pu}$ agree over most of the energy range with the results of the evaluation of Manero and Konshin. However, the present results are larger for ${ }^{235} \mathrm{U}$ than those of Manero and Konshin, and for ${ }^{233} \mathrm{U}$ the present data yield a different energy dependence in the interval 100 to $600 \mathrm{keV}$. Final results are displayed.

- Research sponsored by U.S. DOE OTive of Reactor Reacarch and Technology.

$f_{\text {Inatrumentation and Controls Divition. }}$ 
15

\section{MEASUREMENTS OF THE NEUTRON FISSION CROSS SECTIONS OF ${ }^{225} \mathrm{U}\left(E_{z}=0.01 \mathrm{eV}\right.$ TO $\left.30 \mathrm{keV}\right)$ AND ${ }^{20} \mathrm{~Pa}\left(E_{a}=0.01\right.$ TO $\left.60 \mathrm{eV}\right) *$ \\ R. Grin R. R. Spencer R. W. Inglet J. H. Todd $t$ S. W. Scolest \\ [Abstract of Nucl. Sci Eng. 88, 37 (1984)]}

Measurements have been made of the energy dependence of the ${ }^{235} \mathrm{U}$ neutron fission cross section over the energy range from $0.01 \mathrm{eV}$ to 30 $\mathrm{keV}$ and of the ${ }^{239} \mathrm{Pu}$ fission cross section over the range from 0.01 to $60 \mathrm{eV}$. The energy integral of the fission cross section for ${ }^{235} \mathrm{U}$ was normalized to $19.26 \mathrm{beV}$ in the $0.0206-$ to $0.0639-\mathrm{eV}$ interval; this yielded a value of $248 \pm 1.7 \mathrm{beV}$ for the 7.8- to 11-eV interval, which is in good agreement with other measurements normalized in the same manner. The energy integral for ${ }^{239} \mathrm{Pu}$ was normalized to $25.15 \mathrm{breV}$ in the 0.02001 - to $0.06001-\mathrm{V}$ interval; the resulting value of $504 \mathrm{beV}$ in the 9 - to $12.6 \mathrm{eV}$ interval was also in good agreement with other data. For the energy ranges covered, the energy dependence of both the ${ }^{235} \mathrm{U}$ fission cross section and the ${ }^{239} \mathrm{Pu}$ fission cross section is consistent with ENDF/B-V data except for a few energy intervals in which the ${ }^{235} \mathrm{U}$ cross section differs by as much as $4 \%$.

- Research sponsored by U.S. DOE OfIice of Reactor Research and Technolozy.

$f_{\text {Instrumentation and Controls Division. }}$

tSummer research participant.

1.6

\section{SUBTHRESHOLD FISSION CROSS SECTION OF ${ }^{200}$ PU AND THE FISSION CROSS SECTIONS OF ${ }^{235} \mathrm{U}$ AND ${ }^{23 P}$}

\section{W. Weatoes J. H. Todd}

[Abstract of Nucl. Sci. Eng. 88, 567 (1984)]

The subthreshold fission cross section of ${ }^{240} \mathrm{Pu}$ was measured relative to the ${ }^{16} B(n, \alpha)$ or the
${ }^{6} \mathrm{Li}(n, \alpha)$ cross section from $20 \mathrm{eV}$ to $100 \mathrm{keV}$. Resonance parameter fits to the data were derived from 20 to $5700 \mathrm{eV}$. Many more resonances and more resonance structure were observed than in previous measurements. During the course of this measurement, the fission cross sections of ${ }^{235} \mathrm{U}$ and ${ }^{239} \mathrm{Pu}$ were also measured and are compared to the ENDF/B-V evaluation. The ${ }^{235} \mathrm{U}$ experiment is in good agreement with ENDF/B-V; however, the ${ }^{239} \mathrm{Pu}$ measurement is lower than ENDF/B-V and many of the previous measurements, particularly above $25 \mathrm{keV}$.

-Research sponsored by U.S. DOE Division of Breeder Tect. notogy.

'Instrumentation and Controls Division.

1.7

\section{NATURE OF THE COUPLING IN SUBTHRESHOLD FISSION OF ${ }^{230} \mathrm{Np*}$}
G. F. Auchampanght
J. D. Moses ${ }^{t}$
M. S. Moore ${ }^{\dagger}$
R. C. Extermana ${ }^{t}$
R. O. Nekont
N. W. Hirt
C. E. Obean ${ }^{t}$
J. A. Harvey

[Abstract of Phys. Rev. C 23(1), 174 (1984)]

High-resolution measurements of total and fission cross sections for neutrons on ${ }^{237} \mathrm{~Np}$ in the region of the subthreshold fission structures near 40 and $120 \mathrm{eV}$ have been made with samples cooled to liquid-nitrogen temperature. The previously accepted normalization of the fission cross section is found to be too low by a factor of 3. Extensive one- and two-fission-channel multilevel R-matrix fits to the data and statistical analyses of the fine structure at 40 and $120 \mathrm{eV}$ fail to differentiate between the two proposed coupling schemes in ${ }^{238} \mathrm{~Np}$ : very weak coupling of the class I states to a narrow class II state or moderately weak coupling of the class I states to a broad class II state.

\footnotetext{
- Research sponsored by U.S. DOE Orfice of High Energy and Nuclear Physica.

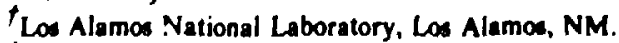

Finstrumentation and Controls Division.
} 
1.8

\author{
NEUTRON-INDUCDD FISSION \\ CROSSSECTION MTASUREMITNTS \\ $\mathrm{OF}^{24 \mathrm{C}},{ }^{24} \mathrm{Cm}, \mathrm{AND}^{2 \mathrm{C}}$ *
H. T. Magrire, Jr. ${ }^{\dagger}$
R. C. Blacts
R. E. Sloncel'
R. J. Dongan**
R. W. Longed
C. R. S. Stopat
D. R. Harrits
J. W. T. Daln
R. W. Hofi**

[Abstract of Nucl. Sci. Eng. 89, 293 (1985)]

The fission cross sections of ${ }^{244} \mathrm{Cm},{ }^{246} \mathrm{Cm}$, and ${ }^{248} \mathrm{Cm}$ have been measured from $0.1 \mathrm{eV}$ to 80 $\mathrm{keV}$ using the Rensselaer Intense Neutron Spectrometer. The cross sections were normalized to the ${ }^{235} \mathrm{U}$ ENDF/B-V broad-binaveraged fission cross section. Fission areas and widths were determined for the resolved lowenergy resonances. In general, the ENDF/B-V fission cross sections for the curium isotopes are in poor agreement with the measured cross sections, and 2 new evaluation of these curium cross sections is recommended. The observation of structure in the measured cross sections in the unresolved region is suggestive of intermediate structure in the even curium isotopes.

-Research sponsored by U.S. DOE OITroe of Bacic Energy Sciences.

Westinghouse Nuclear Technotogy Division, Weatingbouse Nuclear Center-47I, Moaroeville, PA.

F Centro T ́crico Aeroespecial, Instituto de Eatudos Avancados Caixa Portal 6044, 12200 - Sto Jore dos Campon, Brazil.

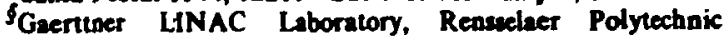
Institute, T.oy, NY.

Knolls Alomic Power Laborstory, Niskayuan, NY.

- Lawrence Livermore National Laboralory, Livermore, CA.

1.9

\section{DETERMINATION OF UNBOUND STATES IN ${ }^{35}$ S FROM NEUTRON TOTAL AND CAPTURE CROSSSECTION MEASUREMENTS ON ${ }^{34}$ *}
R. F. Cartion ${ }^{\dagger}$
W. M. Goodt
J. A. Harvey
R. L. Macklio

B. Castef

[Abstract of Phys. Rev, C 23(6), $1980(19$ )]

The high neutron-resolution capability of the Oak Ridge Electron Linear Accelerator has been used to investigate the unbound region of ${ }^{35} \mathrm{~S}$ via neutron resonance spectroscopy studies of the ${ }^{34} \mathrm{~S}$ $+n$ system. The total cross section of ${ }^{4} \mathrm{~S}$ was measured over the neutron energy range from 90 $\mathrm{keV}$ to $1.5 \mathrm{MeV}$, and the capture cross section was measured over the range from 30 to 1100 keV. Analysis of the data yielded spectroscopic factors for the s-states in the unbound region for excitation energies from 7 to $8 \mathrm{MeV}$. Continuum shell-model calculations of the s- and d-states were also performed for both the bound and the unbound regions of ${ }^{35} \mathrm{~S}$. In general, the measured and calculated neutron strengths for s-waves in the unbound region are in reasonable agreement, although the fragmentation of single-particle strength seen experimentally is somewhat higher than that predicted. Core-particle calculations for the p-states in the unbound region of ${ }^{35} \mathrm{~S}$ were also performed, and, on the basis of all these results, plus those of others, we have summarized our understanding of the distributions of singleparticle neutron strengths in the s-, p-, and $d$-states of ${ }^{33} \mathrm{~S}$ for excitation energies up to 8.5 $\mathrm{MeV}$.

-Reacarch sponsored by U.S. DOE Office of Nuctear Sciencea. Middle Teanenee State Univeraity, Murfreesboro, TN.

tPhyics Division.

${ }^{\$}$ Queens University, Kingron, Canada.

1.10

\section{NEUTRON CAPTURE AND TOTAL CROSS SECTIONS FOR ${ }^{\circ}$ : ASTROPHYSICAL MPLICATIONS*}
R. F. Cartiont
J. A. Harrey
N. W. Hillt
R. L. Mactio

IAbatract of paper prevented it the Fifth International Symposium on Capture Gemma-Ray Spectroacopy and Related Topica, Knoxville, TN, September 10-14, 1984; Proc. p. 774 (1984)]

Attempts to understand abundance anomalies of the $\mathrm{Ca}$ isotopes in the Allende meteorite via the $n, \beta$-process require $30-k e V$ Maxwellianaveraged capture cross sections. Experimental data on ${ }^{48} r_{a}$ in this energy region of astrophysical significance are important since a single resonance in this vicinity could dominate the capture cross section. Neutron capture and 
total cross-section measurements have been performed at ORELA on a 9.97-g sample of $\mathrm{CaCO}_{3}$, enriched to $96 \%{ }^{48} \mathrm{Ca}$, over the energy ranges $10 \mathrm{eV}-500 \mathrm{keV}\left(\sigma_{\gamma}\right)$ and $10 \mathrm{keV}-4 \mathrm{MeV}$ $\left(\sigma_{t}\right)$. Only two small resonances were found for ${ }^{48} \mathrm{Ca}$ in the 30 -keV energy region (at 19.3 and $106.9 \mathrm{keV}$ ) and those only in capture. Their contribution to the $30-k e V$-averaged cross section is only $50 \mu \mathrm{b}$ compared to $1.0 \mathrm{mb}$ calculated from direct capture. The $p$-wave strength and large $d_{S / 2}$ strength observed above $150 \mathrm{keV}$ do not contribute significantly to the $30-k e V$ capture.

- Research sponsored by U.S. DOE Ofice of Basic Energy Sciences.

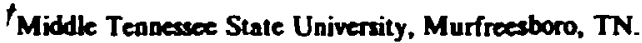

Finstrumentation and Controls Division.

\subsection{1}

\section{Ni + " TRANSMISSION, CAPTURE AND DIFFERENTIAL ELASTIC SCATTERING DATA ANALYSIS IN THE RESONANCE REGION*}
C. M. Perey
F. G. Perey
J. A. Harrey
N. W. Hill ${ }^{\dagger}$

R. L. Macklia

(Abstract of paper presented at the International Conference on Nuclear Data for Basic and Applied Sciences, Santa Fe, NM, May 13-1- 1985)

High resolution neutron transmission, capture and differential elastic-scattering measurements have been made for ${ }^{58} \mathrm{Ni}$-enricher! targets at the Oak Ridge Electron Linear Accelerator from the eV to the MeV region. The three sets of data have been analyzed simultaneously from 10 th $450 \mathrm{keV}$. The analysis of the transmission and differential elastic-scattering data was carried up to $650 \mathrm{keV}$. Averaged parameters were deduced for the $\$ 2 \mathrm{~s}$-wave resonances observed between 10 and $650 \mathrm{keV}$. The average level spacing, $D_{0}$, and the strength function, $S_{0}$, were found to be equal to $12.2 \pm 1.0 \mathrm{keV}$ and $(3.1 \pm 0.6) \times 10^{-4}$, respectively. The differential elastic-scattering data allow us to assign both spin and parity to most of the large non-s-wave resonances which have been observed in the transmission data.

-Research sponsored by U.S. DOE Office of Basic Energy Sciences.

'Instrumentation and Controls Division.

1.12

\section{MEASUREMENTS OF THE NEUTRON TRANSMISSION AND CAPTURE CROSS SECTIONS IN ${ }^{204}$ Pb*}
D. J. Horeat
R. L. Macklia
J. A. Harrey
N. W. Hinf

[Abstract of Physical Review C 29, 2126 (1984)]

High resolution neutron transmission measurements have been performed on ${ }^{204} \mathrm{~Pb}$ in the energy interval $E=0.4-105 \mathrm{keV}$. The transmission data were analyzed using a multilevel R-matrix code to deduce resonance parameters. Previously obtained neutron capture data were reanalyzed in the interval $2.6-86 \mathrm{reV}$. Values of $G \Gamma_{n} \Gamma_{\gamma} / \Gamma$ were determined from the capture data. For those resonances where $\Gamma_{n}$ could be determined from the transmission data, the capture data were analyzed to extract $\Gamma_{\gamma}$. Our results yield an average capture for a stellar temperature $k T=30 \mathrm{keV}$ of $89.5 \pm 4.5 \mathrm{mb}$. The s-wave level density for ${ }^{205} \mathrm{~Pb}$ corresponding to the neutron energy range investigated (i.e., $E-105 \mathrm{keV}$ ) relative to that for ${ }^{207} \mathrm{~Pb}$ (which has about the same neutron separation energy) is greater by about a factor of 10 . The average $s$-wave strength function in this energy region is determined as $S_{o}=0.93 \times 10^{-4}$. This is an order of magnitude greater than that for a similar energy region in ${ }^{206} \mathrm{~Pb}+n$ where a doorway is observed at $E \sim 500 \mathrm{keV}$. However, the strength function in the initial $E=0-100 \mathrm{keV}$ in ${ }^{204} \mathrm{~Pb}+n$ is almost identical to the average value of that for ${ }^{206} \mathrm{~Pb}+n$ when the averaging interval for the latter is taken as $E_{n} \approx 0-1000 \mathrm{keV}$ (i.e., over the doorway state). This suggests that the s-wave doorway state observed in the higher mass lead 
isotopes is completely mixed with background" states in ${ }^{205} \mathrm{~Pb}$, and most likely no intermediate structure will be observed in the s-wave strength function for the ${ }^{201} \mathrm{~Pb}+n$ reaction.

- Research sponsored by US. DOE OTice of Basic Energy Sciences.

'Physics Division.

$t_{\text {Instrumentation and Controls Division. }}$

\subsection{3}

\section{NEUTRON CAPTURE BY ${ }^{31}$ P*}

\section{R. L.Mnction S. F. Mophalydubt}

[Abstract of Phys. Rev. C 32, 379 (1985)]

Resonance parameters for ${ }^{31} \mathrm{P}+n$ were determined, largely from $(n, \gamma)$ cross-section data measured by time of flight at the Oak Ridge Electron Linear Accelerator facility. The energy range investigated extended from $2.6 \mathrm{keV}$ to 500 $\mathrm{keV}$, with the lowest energy resonance found at $26.75 \mathrm{keV}$. The $30-\mathrm{keV}$ stellar reaction rate is dominated by this resonance, giving $1.74 \pm 0.09$ $m b$ for a temperature $k T=30 \mathrm{keV}$. The thermal capture data are examined within the framework of the direct reaction mechanism.

- Research sponsored by U.S. DOE OTIice of Batic Enerry Sciences.

Broothaven National Leboratory, Upton, NY.

\subsection{4}

\section{RESONANCE NEUTRON CAPTURE} BY ${ }^{36,37} \mathrm{C} *$

\section{R. Lo Macklin}

[Abetract of Phys. Rev. C 294(6h 1996 (1984)]

Neutron rapture by enriched ${ }^{37} \mathrm{Cl}$ and by natural C) was measured at the Oak Ridge Electron Linear Accelerator (ORELA) as a function of neutron time of flight over a 40-meter path. Resonance peaks were fitted by least squares to Breit-Wigner parameters. The energy range covered was 4 to $225 \mathrm{keV}$ for $54{ }^{35} \mathrm{Cl}$ resonances and 8 to $151 \mathrm{keV}$ for $12{ }^{37} \mathrm{Cl}$ resonances Corresponding average capture in stellar environments at $k T=30 \mathrm{keV}$ is calculated as $(10.0 \pm 0.3) \mathrm{mb}$ for ${ }^{35} \mathrm{Cl}$ and (2.15 $\pm 0.08) \mathrm{mb}$ for ${ }^{37} \mathrm{Cl}$.

-Reacarch sponorod by U.S. DOE Ofrice of Baxic Enery Sciences.

\subsection{5}

\section{RESONANCE NEUTRON CAPTURE BY ${ }^{32,41} \mathrm{~K}$ *}

\section{R. L Mnctin}

\section{[Abatract of Nucl. Sci. Eng. 88, 129 (1984)]}

Neutron capture by a sample enriched in ${ }^{11} \mathrm{~K}$ and by a natural $K$ sample $\left(93.26 \%{ }^{39} \mathrm{~K}\right.$ ) was measured at the Oak Ridge Electron Linear Accelerator (ORELA) as a function of neutron time of flight over a 40-m flight path. Resonance peaks were fitted by a least-squares fitting program to obtain Breit-Wigner resonance parameters. The energy range extended to all resonances below $153 \mathrm{keV}$. Corresponding average capture at a temperature typical of stars, $k T=30 \mathrm{keV}$, is calculated as $(11.8 \pm 0.4) \mathrm{mb}$ for ${ }^{39} \mathrm{~K}$ and $(22.0 \pm 0.7) \mathrm{mb}$ for ${ }^{41} \mathrm{~K}$.

- Reacarch sponecored by U.S. DOE OMTroe of Basic Eneryy Sciences.

1.16

\section{RESONANCE NEUTRON CAPTURE BY MANGANESE BELOW 25 keV*}

\section{R. L. Mact lit}

[Abatrect of Nmcl. Sct. Eng. 89, 362 (1985)]

Radiation widths $\Gamma_{\gamma}$ of $(310 \pm 20),(312 \pm$ 12), ald (340 \pm 130$) \mathrm{meV}$ were found for ${ }^{35} \mathrm{Mn}(n, \gamma)$ resonances at 337,1099 , and $2327 \mathrm{eV}$, respectively. A fourth resonasce was found at $1658 \mathrm{eV}$, with $g \Gamma_{n} \Gamma_{\sqrt{ }} \Gamma-(7.6 \pm 0.3) \mathrm{meV}$.

-Research sponeored by U.S. DOE Orice of Bacic Energy Sciences. 
1.17

\section{CAPTURE IN THE 1.15-teV IRON RESONANCE*}

\author{
L. W. Weaton J.H. Tout
}

(Abstract of paper presented at the International Conference on Nuclear Dala for Basic and Appliod Sciences, Sunta Fe. NM, May 13-17, 1985)

The 1.15-keV resonance in iron is important to reactors in that a major part of the iron capture integral is due to this one resonance. This resonance is also a good test case for capture gamma-ray detectors, which are meant to have an efficiency independent of gamma-ray energy spectra, because this resonance has an unusually hard spectrum and other iron resonances do not. There have been severe discrepancies among differert groups who have measured the capture area and transmission of this resonance. A new measurement, employing $\mathrm{C}_{6} \mathrm{~F}_{6}$ liquid scintillators and pulse-height weighting to effect total energy detectors, has been made of the ratio of the capture area of the 22.8-keV resonance relative to that of the 1.15-keV resonance. The 22.8-keV resonance has a relatively soft gamma-ray spectrum and there is agreement on the capture area. The results $(2.91 \pm 0.17)$ agree well with transmission measurements and confirm the applicability of total energy detectors for use on capture resonances with very hard capture gamma-ray spectra.

- Revearch sponsored by U.S. DOE OfTxce of Basic Enetgy Sciences.

$t_{\text {Instrumentation and Controls Division. }}$

\subsection{8}

\section{NEUTRCN CAPTURE MEASUREMENTS ON RADIOACTTVE ${ }^{93} \mathrm{Z}$ *}

\section{R. L. Macklia}

[Abstract of Astrophysics and Space Science 115, 11 (1985)]

Neutron capture measurements made on a sample of fission-product zirconium containing $20{ }^{93} \mathrm{Zr}\left(t^{1 / 2}=1.5 \times 10^{6}\right.$ a $)$ at the Oak Ridge
Electron Linear Accelerator time-of-flight facility resulted in the identification of 138 resonance peaks for the ${ }^{93} \mathrm{Zr}$ isolope at neutron energies up to $21.5 \mathrm{keV}$. Average capture cross sections from $20 \mathrm{keV}$ to $300 \mathrm{keV}$ were derived by subtracting neutron capture yields of the stable zirconium isotopes $90.91 .9294 .96 \mathrm{Zr}$ and additional backgrounds. The average cross sections found were significantly less than those of JENDL-1. While generally $30 \%$ higher than those of ENDF/B-V below $60 \mathrm{keV}$, the binned data overlapped the smooth ENDF/B-V curve. The average for a Maxwellian neutron spectrum with $k T=30 \mathrm{keV}$ is $(95 \pm 10) \mathrm{mb}$, and the resonance contribution to the capture resonance integral is $(15.0 \pm 0.5)$ b.

- Research sponsored by U.S. DOE Office of Basic Enerzy Sciences.

1.19

\section{NEUTRON CAPTURE MEASUREMENTS ON FISSION PRODUCT PALLADIUM-107*}

\section{R. L. Macklia}

[Abstract of Nucl. Sci. Eng. 89, 79 (1985)]

Neutron capture measurements were made on a sample of fission-product palladium at the Oak Ridge Electron Linear Accelerator time-of-flight facility. One hundred thirty resonance peaks were parameterized up to $3.5 \mathrm{keV}$ and the average cross section from 3 to $600 \mathrm{keV}$ was derived. The data exceed the ENDF/B-V evaluation by about $25 \%$ in the 3- to $300-\mathrm{keV}$ range but drop steeply below it at higher energies where neutron inelastic-scattering competition becomes important. The Maxwellian average cross section for $k T=30 \mathrm{keV}$ is calculated as $1.34 \pm 0.06 \mathrm{~b}$, and the dilute resonance capture integral as $108.1 \pm 4.3 \mathrm{~b}$.

-Research sponsored by U.S. DOE Orrice of Basic Energy Sciences. 
1.20

\section{NEUTRON CAPTURE CROSS SECTIONS AND SOLAR ABUNDANCES OF ${ }^{16,161}$ Dy, ${ }^{170,171 Y}$ Y, ${ }^{173,17 \%} \mathrm{LE}$, AND ${ }^{176,17} \mathrm{H}$ \\ FOR THE s-PROCESS ANALYSIS OF THE RADIONUCLIDE ${ }^{17} \mathrm{Ln}$}

\author{
H. Beert \\ G. Walter ${ }^{t}$ \\ R. L. Mactlia \\ P. J. Patchetet
}

[Abatract of Phys. Rev. C 34(2), 464 (1984)]

The neutron capture cross sections and solar abundances of ${ }^{160,161} \mathrm{Dy},{ }^{170,171} \mathrm{Yb},{ }^{175,176} \mathrm{Lu}$, and 176,17 Hf have been measured. With this data base s-process studies have been carried out to determine the s-process neutron density and temperature and to investigate the s-process nucleosynthesis of the ${ }^{176} \mathrm{Lu}$ clock. From various branchings the neutron density was found to be $(0.8-1.8) \times 10^{3}$ neutrons per $\mathrm{cm}^{3}$ and the temperature $k T$ to be $18-28 \mathrm{keV}$. On the basis of the present data, ${ }^{176} \mathrm{Lu}$ proved not to be applicable as a cosmic clock because of the temperature sensitivity of the ${ }^{176} \mathrm{Lu}$ half-life but can be used instead as a stellar thermometer. Constraints for the s-process temperature ( $k T=$ $20-28 \mathrm{keV}$ ) were found to be in $\operatorname{good}$ agreement with the investigated branchings.

-Research sponsored by U.S. DOE Office of Basic Enetgy Sciences.

'Kernforacbungaxentrum Karlsrube, Institut Fur Kernphysik, Federal Republic of Germany.

IMax Planct-Institut fus Chemic, D-6500 Mainz, Foderal Republic of Germany.

\subsection{1}

\section{THE ${ }^{163}$ Dy- ${ }^{163} \mathrm{Ho}$ BRANCHING: AN s-PROCESS BAROMETER*}

\section{H. Beer ${ }^{\dagger}$ G. Walter ${ }^{\dagger}$ \\ R. L. Mnethio}

[Abatract of paper presented at the Fifth International Symposium on Capture Gamma-Ray Spectroncopy and Related Topics, Knoxville. TN, September 10-14, 1984; Proc. p. 778 (1984)]

The neutron capture cross sections of ${ }^{163} \mathrm{Dy}$ and ${ }^{164} \mathrm{Er}$ have been measured to analyze the s-process branching at ${ }^{163} \mathrm{Dy}-{ }^{163} \mathrm{Ho}$. The reproduction of the s-process abundance of ${ }^{164} \mathrm{Er}$ via this branching is sensitive to temperature $k T$, neutron density, and electron density $n_{e}$. The calculations using information from other branchings on $k T$ and the neutron density $n_{n}$ give sonstraints for $n_{e}$ at the site of the s-process.

- Research sponsored by U.S. DOE Office of Nuctear Science.

'Kernforschungazentrum Karlsrube, Institut FUr Kernphysik, Federal Republic of Germany.

1.22

19.19,200,201,202,204/H(r,r) CROSS SECTIONS
AND THE TERMINATION OF
3-PROCESS NUCLEOSYNTHESIS*

\section{H. Beer ${ }^{t}$ R. L. Mactlin}

[Abatract of Phys. Rev. C 32(3), 738 (1985)]

The neutron capture cross sections of 198.199,200,201,202,28 ${ }^{4} \mathrm{Hg}(n, \gamma)$ were measured in the energy range 2.6 to $500 \mathrm{keV}$. The average capture cross sections were calculated and fitted in terms of strength functions. Resonance parameters for the observed resonances were determined by a shape analysis. Maxwellian averaged capture cross sections were computed for thermal energies $k T$ between 5 and $100 \mathrm{keV}$. The solar mercury abundance was determined to be $0.34 \pm 0.04$ relative to $\mathrm{Si}=10^{6}$. The termination of s-process nucleosynthesis at lead and bismuth was investigated. The abundances of $206,207.208 \mathrm{~Pb}$ were reproduced introducing a strong fluence component of the s-process in addition to normal $s$ - and r-process nucleosynthesis. The radiogenic ${ }^{201} \mathrm{~Pb}$ abundance was determined and the r-process age was calculated via ${ }^{235} \mathrm{U}$. Using Fowler's exponential model, an age $T=4.6 \mathrm{Gyr}+\Delta=17.2 \pm 2.6$ Gyr was obtained.

- Research aponsored partially by U.S. Department of Encrey.

${ }^{\prime}$ Kernforachungazentrum Karlaruhe, Institut Fur Kernphyzik, Federal Republic of Germany. 


\section{NEUTRON CAPTURE CROSS SECTION OF AMERICTUM-243*}

\author{
L. W. Weaton J. H. Todd \\ [Abstract of Nucl. Sci Eng. (in press)]
}

The neutron capture cross section of ${ }^{243} \mathrm{Am}$ was measured from $258 \mathrm{eV}$ to $92 \mathrm{keV}$. The relative capture cross sections were normalized to $74.8 \mathrm{~b}$ at $0.0253 \mathrm{eV}$ from ENDF/B-V. Agreement with the one previous measurement in the $\mathrm{keV}$ neutron energy range is reasonably good $(-8 \%)$. These results are needed for calculating the buildup of higher actinides in operating reactors.

-Research sponsored ty, U.S. Department of Exergy.

Instrumentation \& Controls Division.

\subsection{4}

s- AND p-WAVE NEUTRONS ON 39Si AND ${ }^{34}$ S: SPHERICAL OPTICAL MODEL ANALYSIS*

\section{R. F. Carlton $\quad$ J. A. Harrey \\ C. H. Johnsont}

[Abstract of Phys. Rev. C 29, 1988 (1984)]

The $s$ - and $p$-wave neutron scattering functions obtained previously by R-matrix analyses of high resolution transmission data for 0 - to $1.4-\mathrm{MeV}$ neutrons on ${ }^{30} \mathrm{Si}$ and ${ }^{34} \mathrm{~S}$ have been averaged by means of a simple analytic approximation to obtain experimental optical model scattering functions. To describe these with a spherical optical model potential requires the real well depth to be about $20 \%$ deeper for $p$-waves than for s-waves. This result agrees with an carlier analysis for ${ }^{32} \mathrm{~S}$ and suggests that deformation effects must be included for all three nuclei.

- Research sponsored hy U.S. DOE Office of Basic Energy Sciences.

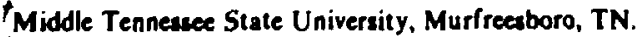

IPhysia Division.

\section{NEUTRON, ALPHA AND TOTAL WIDTHS AND SPIN ASSIGNMENTS FOR RESONANCTS IN ${ }^{33}+a$ FROM $10-400$ keV*
G. P. Collenst
M. Salant
N. W. HFins \\ J. A. Harrey}

[Abstract of paper presented at the Neutron Nucleus Collisions Cooference, Glouster, OH, September 5-8, 1984; Proc. AIP Conf. No. 124, p. 302 (1985)]

High-resolution transmission measurements on a $0.97-\mathrm{g}$ sample enriched to $88.29 \%{ }^{33} \mathrm{~S}(\mathrm{I}=$ $3 / 2^{+}$) were made at ORELA using an NE-110 scintillation detector. Transmission data on this nuclide are of special interest since some of the resonances can have large alpha widths and the $(n, \alpha)$ cross section has been measured recently at GEEL. The multilevel R-matrix formalism (including an external R-function) was used in analyzing the data by a computer code SAMMY based on Bayes' Theorem. Three exit channels $\left(\Gamma_{n}, \Gamma_{\gamma}\right.$, and $\left.\Gamma_{a}\right)$ were taken into account in the analysis. The $2^{+}$states produced by $s$-wave neutrons and $1^{-}$and $3^{-}$states produced by $p$-wave neutrons can decay by alpha emission, whereas $1^{+}, 0^{-}$, and $2^{-}$states do not. Neutron strength functions and level spacings for s- and p-wave neutrons were obtained.

- Research sponsored by the Belgian Nationaal Fonds voor Wetenschappelijk Onderzoek and by the U.S. DOE Office of Basic Energy Sciences.

'Universitaire Instelling Antwerpen, Antwerp, Belgium.

t Minia University, El Minia, Egypt.

$\delta_{\text {Instrumentation and Controls Division. }}$

\subsection{6}

\section{$d_{\text {S/2 SINGLE PARTICLE }}$ STRENGTH IN *Ca $+n^{*}$}

\section{J. A. Harrey C. H. Johnoont \\ R. F. Cartton B. Castef}

IAbstract of paper presented at the Neutron Nucleus Collisions Conference, Glouster, OH, September 5 8, 1984; Proc. AIP Conf. No. 124, p. 304 (1985)]

The neutron total cross section of ${ }^{48} \mathrm{Ca}$ was measured up to $4 \mathrm{MeV}$ and the data were 
analyzed using an R-matrix code to obtain resonance parameters and potential scattering phase shifts. No s-wave resonances were observed, and the small cross section $(-0.5$ b) at low energy requires a real well depth of 48 MeV. Three strong $d$-wave resonances (amounting to $45 \%$ of the single particle iimit) were found in the $0.8-$ to $2.0-\mathrm{MeV}$ energy region. Shell-model-in-the-continuum calculations agree with these observations.

-Research spossored by U.S. DOE OMToe of Besic Enerny Sciences.

tPhysia Division.

F Middle Tendessoe State University. Murfreatoro, TN.

'Queens' University, Kingroa, Canada.

1.27

\section{SINGLE-PARTICLE 2d/2 STRENGTH IN THE Ca + REACTION*}

\section{J. A. Harrey \\ C. H. Johnsoot \\ R. F. Carttont \\ B. Casteff}

[Abotract of Phys. Rev. C Rapid Communications 3243), 1114 (1985)]

The neutron total cross section of ${ }^{48} \mathrm{Ca}+n$ has been measured up to $10 \mathrm{MeV}$ and analyzed up to $4 \mathrm{MeV}$ using the R-matrix formalism to obtain resonance parameters and potential scattering phase shifts. Very little s-wave neutron strength was observed, and the small cross section $(0.5 \pm 0.2$ b) observed for low-energy neutrons ( $<150 \mathrm{keV})$ can be described by a real WoodsSaxon potential with $V_{0}=47.3 \pm 0.7 \mathrm{MeV}, r_{0}$ $=1.21 \mathrm{fm}$, and $a=0.66 \mathrm{fm}$. Three strong $d_{5 / 2}$ resonances amuunting to $45 \%$ of the singleparticle width were found in the 0.8- to $2.0-\mathrm{MeV}$ energy region. These results compare well with cross-section predictions from two microecopic calculations. The $p_{1 / 2}, p_{3 / 2}$, and $d_{3 / 2}$ reconance strengths are very weak $\left(<<\right.$ than the $d_{s / 2}$ strength).

- Research sponsored by U.S. DOE Orince of Basic Enerry Sciences.

'Physica Division.

I Middle Tenneace State University, Murfreaboro, Tenneaces.

'Quoen's University, Kingaton, Ceneds.
1.28

\section{PARITY DEPENDENCE OF THE LEVEL DENSTITISS OF ${ }^{50}$ C AND ${ }^{50}$ Cr AT HIGH EXCTTATION*}

\author{
$\begin{array}{ll}\text { H. M. Agrawal } & \text { J. B. Garg }\end{array}$
}

J. A. Harvey

[Abaract of Phyx. Rev. C 340, 1880 (1984)]

The neutron total cross sections of ${ }^{52} \mathrm{Cr}$ and ${ }^{54} \mathrm{Cr}$ have been measured in the energy range irom a few tens of keV 10 about $900 \mathrm{keV}$ using a neutron time-of-flight technique and a pulsed electron linear accelerator. The nominal resolution of the measurements was about 0.06 $\mathrm{ns} / \mathrm{m}$. The total cross-section data have been analyzed using an R-matrix multilevel multichannel code to determine values of the resonance parameters $\left(E_{0,} g \Gamma_{n}\right.$, and $\left.F\right)$ and to investigate the parity dependence of the level densities of ${ }^{53} \mathrm{Cr}$ and ${ }^{55} \mathrm{Cr}$ at high excitation. From these analyses we obtain the following values for the average properties of the resonance parameters for s-wave resonances up to about 900 $\mathrm{keV}$ and for p-wave resonances up to $600 \mathrm{keV}$ : $D_{0}=(45 \pm 6) \mathrm{keV}, S_{0}\left(\times 10^{4}\right)=(3.0 \pm 1.0)$, $D_{1}=(8.5 \pm 0.6) \mathrm{keV}, S_{1}\left(\times 10^{4}\right)=(0.70 \pm$ 0.12) for ${ }^{52} \mathrm{Cr}$, and $D_{0}=(60 \pm 9) \mathrm{keV}$, $S_{0}\left(\times 10^{4}\right)=(2.6 \pm 0.9), D_{1}=(9.2 \pm 0.5)$ keV, $S_{1}\left(\times 10^{4}\right)=(0.67 \pm 0.11)$ for ${ }^{54} \mathrm{Cr}$. The distributions of reduced neutron widths for $s$ - and p-wave resonances for each isotope show good agreement with the Porter-Thomas distribution. The values of the $\Delta_{3}$ statistic for the long-range correlation of s-wave resonances for both ${ }^{52} \mathrm{Cr}$ and ${ }^{44} \mathrm{Cr}$ are found to be in reasonable agreement with the theoretical prediction of Dyson and Mehta. The values for the ratio $D_{0} / D_{1}$ for both nuclidea are larger than expected from a $(2 J+1)$ level dependence, indicative of a parity dependence of the level densities of ${ }^{53} \mathrm{Cr}$ and ${ }^{53} \mathrm{Cr}$ at high excitation.

\footnotetext{
- Research sponsored by U.S. DOE Division of Nucleas Sciences.

'State University of New York, Albany NY.
} 
1.29

OPTICAL MODEL SCATTERING FUNCTIONS

R. F. Cartioa ${ }^{\dagger}$ J. A. Harrey

C. H. Jnweont

IAbatract of paper prosented at the Neutron Nuctars Collisions Coaference. Glouster. OH. September 5-8. 1984; Proc AIP Coal. No. 124. p. 308 (1985)]

Using the ORELA 200-m time-of-flight facility, we measured the neutron total cross section with good resolution for a 96\%-enrichad ${ }^{26} \mathrm{Kr}$ target and made a multilevel R-matrix analysis up to $800 \mathrm{keV}$. By averaging we deduced optical scattering functions for each partial wave and fit them by adjusting well depths in a spherical model with our standard geometry. Our preliminary best fit for the real wells has $V_{0}=48 \mathrm{MeV}$ for $l=0, V_{0}=52 \mathrm{MeV}$ for $\ell=1$, and $V_{s o}=6 \mathrm{MeV}$. The resulting predicted spin-orbit enhancement of the $p_{3 / 2}$ strength relative to $p_{1 / 2}$ is a ratio of 1.7 , whereas the observed ratio is 3.5. Thus, there appear to be structure effects which can be described in the optical model by $W_{D}=2 \mathrm{MeV}$ for $p_{1,2}$ and $W_{D}$ $=4 \mathrm{MeV}$ for $p_{3 / 2}$.

-Research sponsored by U.S. DOE OTTice of Basic Energy Sciences.

Middle Tennesuce State University, Murfreesboro, TN.

TPhysias Division

1.30

\section{TOTAL CROSS SECTIONS FOR LOW ENERGY NEUTRONS ON $\boldsymbol{w}_{\mathbf{K}}{ }^{*}$ \\ R. F. Cartion' C. H. Johneon't}

\section{J. A. Harrey}

(Abstract of paper presented at the American Physical Society Meeting. Nashvilk, TN, October 18-20, 1984)

Neutron total cross sections have been measured with high resolution at the ORELA 200-m night-path facility for a 99.5\%-enriched ${ }^{86} \mathrm{Kr}$ target $(1 / N=49 \mathrm{~b} / \mathrm{atom})$. Strength functions, level spacings, and potential scattering phase shifts have been reduced from an R-matrix analysis for neutron partial waves up to $\boldsymbol{F}=$ $S / 2^{+}$. Most of the resonance strength is due to p-wave neutrons. That is expected for this nucleus because it lies just below the 3-p size resonance; however, the observed ratio of 3.5 for the $p_{3 / 2}$ relative to $p_{1 / 2}$ strength function is twice that predicted from an optical model with a real spin-orbit term which well describes the energyaveraged scattering function. Optical model parameters will be compared to earlier results for lower mass nuclides.

-Research sponsored by U.S. DOE OTTwe of Basic Energy Sciences.

'Middle Tendessec Stale University. Murfreaboro. TN.

tPhysias Division.

1.31

\section{ICCURATE DETERMINATION OF THE PARAMETERS OF THE 292.4-V RESONANCE OF ${ }^{\prime \prime} \mathrm{Z}$ AND THE 301.3-V RESONANCE OF "Z*}

\author{
M. M. Salaht $\quad$ J. A. Harrey \\ N. W. Hinf A. Z. Hessein
}

F. G. Perey

(Abstract of paper presented at the International Conference on Nuclear Data for Basic and Applied Sciencea, Santa Fe, NM. May 13-17, 1985)

High-resolution transmission measurements of zirconium metal samples have been carried out at ORELA using the $80-\mathrm{m}$ llight path and an improved 'Li-glass scintillation neutron detector. Zirconium is used for reactor fuel cladding and some structural components. Neutron capture by the low-energy resonances is important and resonance parameters from earlier measurements were in disagreement. Four different thicknesses of the pure zirconium metal and one sample of zircalloy were used in four separate expxriments. The transmission data for these samples were measured at room temperature, and one sample was cooled to liquid nitrogen temperature. These data were analyzed using the multilevel $\mathbf{R}$-matrix computer code SAMMY. The resonance 
parameters obtained for this $5-\mathrm{t}$ ave resonance of ${ }^{1} \mathrm{Zr}$ (not including systematic uncertainties) are: $J=3^{+}, E_{0}=29240 \pm 0.10 \mathrm{eV}, \Gamma_{n}=$ $665 \pm \mathrm{MeV}, \Gamma_{\gamma}=131 \pm 7 \mathrm{MeV}$, and $c c\left(\Gamma_{\mathrm{m}}\right.$ $\left.\Gamma_{\gamma}\right)=0.27$. The p-wave reso rance of ${ }^{96} \mathrm{Zr}$ at $311.3 \mathrm{eV}$ with $J=1 / 2$ has been also analyzed and the parameters obtained are: $E_{0}=301.14$ $\pm 0.10 \mathrm{cV}, \Gamma_{z}=223 \pm 7 \mathrm{MeV}, \Gamma_{7}=235$ $\pm 38 \mathrm{MeV}$, and $c c\left(\Gamma_{n}, \Gamma_{\gamma}\right)=080 \mathrm{MeV}$.

- Recearch sponsored by the U.S. Depertrneat of Energy.

tMiaia University, E Minia, Egypt.

IInstrumeatation and Controts Division.

- Alemir Energy Establishmeat. Eopht.

1.32

\section{NEUTRON TRANSMISSION MEASUREMENT AND RESONANCE ANALYSIS OF ${ }^{33} \mathrm{Zr}$ FROM 60 TO 6000 eV*}

\author{
R. L. Macklio J. A. Harrey \\ N. W. Hint
}

[Absursa of Phy. Rev. C (in press)]

Neutron transmission through a zirconium oxide sample containing the radioactive ${ }^{93} \mathrm{Zr}$ isotope was measured at the Oak Ridge Electron Linear Accelerator (ORELA) pulsed neutron time-of-flight facility. R-matrix parameters for some of the resonances up to $6-\mathrm{keV}$ neutron energy were determined by adjustment to the data using the computer code SAMMY. The first resonance, for example, was found at $(110.43 \pm 0.01) \mathrm{eV}$ with partial widths $\Gamma_{n}=$ $(348 \pm 9) \mathrm{MeV}$ and $\Gamma_{\gamma}=(1<1 \pm 21) \mathrm{MeV}$ and quantum numbers $\mathrm{J}^{x}=2^{+}$.

-Reacarch sponsored by U.S. DOE OFTix of Bacic Enerty Sciences.

Instrumentation and Controls Division.
1.33

\section{ORSERYATION OF EXTREMTLLY LOW S-WAVE STRENGTH IN

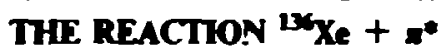

\author{
B. Fegtivert I. A. Harney \\ M. Mire
}

[Aburact of paper preseated at the Fifth Interational Symposium os Capture Gamma-Ray Spoctronoopy and Retaled Topics, Kroxville, TN, September 10-14, 1984; Proc. p. 493 (1984)]

The neutron cross section of ${ }^{136} \mathrm{Xe}$ bas been investigated at the Oak Ridge Electron Linear Accelerator (ORELA). A sample of xenon gas, enriched to $93.6 \%$ is ${ }^{136} \mathrm{Xe}$, was used as the target. Measurements were made with an energy resolution of $\approx 0.1 \%$. The transmission data, which were analyzed for neutron energies below $500 \mathrm{keV}$, show 35 resonances in this region. Only four very weak resonances were found to be possibly due to s-wave neutron interactions, giving an extremely low value for the s-wave strength function in the analyzed region.

-Research sponsored by U.S. DOE OTroc of Besic Enerty Sciences.

The Studsvil Science Research Labocatory, S-611 82, Nytoping. Srroden.

tJapen Alomic Energy Research Institute, Totai-Mura, Naka-guin, Ibaraki-Ken, Japaa.

SPhysics Invision.

\subsection{4}

\section{TEST OF FERMI GAS MODEL PREDICTIONS OF LEVEL DENSITY IN ${ }^{137} \mathrm{Xe}$ "
B. Fogelbers ${ }^{t}$
J. A. Harrey
M. Miranotot
S. Ranas

[Abatract of Phys. Rev. C 31(0, 2041 (1985)]

We have studied the unbound levels of ${ }^{137} \mathrm{Xe}$ via neutron resonance reactions and $\beta$ decay of 
${ }^{137}$ I. High-resolution neutron transmission data revealed a tolal of 35 resonances below a neutron energy of $500 \mathrm{keV}$. At least 27 of the resonances are $p$-wave resonances. The near absence of s-wave resonances in this energy region resulted in an extremely low value for the s-wave neutron strength function. The current $\gamma$-ray data complement earlier studies of $\gamma$ rays and delaysd neutrons following the $\beta$ decay of ${ }^{137} I$. By combining all data, we have obtained a detailed picture of the level density in ${ }^{137} \mathrm{Xe}$ for a wide range of angular momenta. With the exception of $1 / 2^{+}$and $1 / 2^{-}$levels, the overall agreement is good between the current data and predictions of the Fermi gas model.

- Research sponsored by US. DOE Office of Basic Energy Sciences.

tThe Studsvik Science Research Laboratory, S-611 82 , Nytoping. Swoden.

tJapen Atomic Energy Research Institute. Totai-Mura. Naka-gun, Iberaki-Ken, Japan.

Spysics Division.

\subsection{5}

\section{NEUTRON TOTAL CROSS SECTION OF PQ-240 BELOW 6 eV AND THE PARAMETERS OF THE 1.056-V RESONANCE*}

\section{R. R. Spencer J. A. Harvey N. W. Hiil' ${ }^{\dagger}$ L. W. Weaton}

(Abstract of peper presented at the Intermational Conference on Nuclear Dala for Basic and Applied Sciences, Santa Fe, NM, May 13-17, 1985)

Measurements of the transmission of neutrons through Pu metal samples have been made at the Oak Ridge Electron Linear Accelerator (ORELA) to obtain consistency between the parameters of the 1.056-eV resonance and the measured total cross section at $2200 \mathrm{~m} / \mathrm{s}$. Three different thicknesses of Pu metal foils containing 0.727 at. $q$ of Pu-240 were measured at room temperature to obtain the resonance purameters. Assuming the usual Lamb limits for Doppler broadening are satisfied, preliminary analysis of these data resulted in $\Gamma_{n}=0.00244 \pm 0.00002$ $\mathrm{eV}$ and $\Gamma_{\gamma}=0.0300 \pm 0.0002 \mathrm{eV}$. In addition, two sample thicknesses of extremely pure Pu-240 metal (99.97 at.5) were measured from 0.003 to $6 \mathrm{eV}$. Analysis of these data gave a $2200-\mathrm{m} / \mathrm{s}$ total cross section of $284 \pm 2$ barns, which is 2.5\% lower than that calculated from the absorption cross section reported by Lounsbury et al., and M. Lounsbury, R. W. Durham, and G. C. Hanna, Nuclear Data for Reactors, Proceedings of a Conference, Vol. I, p. 287, 1970, from mass spectrometer analyses of Pu samples after irradiation in a thermal column.

-Research spousored by the U.S. DOE Office of Basic Encroy Sciences.

Insarumeatation and Controts Division.

1.36

$$
\begin{aligned}
& \text { FLSSION-PRODUCT YIELDS FOR } \\
& \text { THERMAL-NEUTRON FISSION OF } \\
& { }^{203} \text { CO DETERMINED FROM } \\
& \text { MEASUREMENTS WTTH A HIGH- } \\
& \text { RESOLUTION LOW-ENERGY } \\
& \text { GERMANIUM GAMMA-RAY } \\
& \text { DETECTOR* }
\end{aligned}
$$

\section{D. Merrinan ${ }^{\dagger}$}

(Abstract of ORNL/TM-9049, April 1984)

Cumulative fission-product yields have been determined for 13 gamma rays emitted during the decay of 12 fission products created by thermal-neutron fission of ${ }^{243} \mathrm{Cm}$. A highresolution low-energy germanium detector was used to measu $e$ the pulse-height spectra of gamma rays emitted from a 77-nanogram sample of ${ }^{243} \mathrm{Cm}$ after the sample had been irradiated by thermal neutrons. Analysis of the data resulted in the identification and matching of gamma-ray energies and half-lives to individual radioisotopes. From these results, 12 cumulative fission-product yields were deduced for radionuclides with halflives between $4.2 \mathrm{~min}$ and $84.2 \mathrm{~min}$.

\footnotetext{
-Rerearch sponeored by U.S. DOE Orice of Basic Energy Sciences.

tUniversity of Tennewee, Knoxville, TN.
} 


\section{CALCULATED NEUTRON-INDUCED CROSS SECTIONS FOR "MCSC FROM 1 TO 20 MeV AND COMPARISONS WTTH EXPERIMENTS*}

\author{
D. M. Hetrict $\quad$ C. Y. Fu \\ D. C. Larson
}

[Abstrace of ORNL/TM-9083 (ENDF-337), Aagua 1984]

Nuclear model codes were used to compute cross sections for neutron-induced reactions on both ${ }^{63} \mathrm{Cu}$ and ${ }^{65} \mathrm{Cu}$ for incident energies from 1 to $20 \mathrm{MeV}$. The input parameters for the model codes were determined through analysis of experimental data in this energ. region. Discussion of the models used, the input data, the resulting calculations, extensive comparisons to measured data, and comparisons to the Evaluated Nuclear Data File (ENDF/B-V) for Cu (MAT 1329) are included in this report.

- Research sponsored by the Defense Nuctear Agency.

'Compating and Telocommenications Division.

\section{Evaluations and Reviews}

1.38

\section{CALCULATED NEUTRON-INDUCED CROSS SECTIONS FOR ${ }^{63 \times 5} \mathrm{Ca}_{4}$ saring, AND ${ }^{52} \mathrm{Cr}$ FROM 1 TO $20 \mathrm{MeV}$ AND COMPARISONS WITH EXPERIMINTS*}

\section{M. Hetrict ${ }^{\prime}$ C. Y. Fu}

(Abatract of paper presented at the International Conference on Nuctear Data for Basic and Applied Sciences, Sunta Fe, NM. May 13-17, 1985)

Nuclear model codes were used to compute cross sections for neutron-induced reactions for ${ }^{63,65} \mathrm{Cu}$, ${ }^{58,60} \mathrm{Ni}$, and ${ }^{52} \mathrm{Cr}$ for incident energies from 1 to $20 \mathrm{MeV}$. The optical-model code GENOA and the Distorted Wave Born Approximation program DWUCK were used to determine optical-model parameters and directinteraction cross sections needed as inpit for the Hauser-Feshbach code TNG. The TIJG code simultaneously computes the compor nd and precompound cross sections for all energetically possible binary reactions and tertiary reactions, and computes the resulting gamma-ray production cross sections. Angular momentum is conserved in both compound and precompound reactions. Results from TNG, including energy and angular distributions for particles emitted, are compared with experimental data and are found to agree reasonably well.

- Recearch sponwored by the Defence Nuclear Agency.

'Computing and Telecommunicationa Division.

1.39

\section{PAIRING CORRECTION OF PARTICLE-HOLE STATE DENSITIES FOR TWO KINDS OF FERMIONS*}

\section{Y. Fe}

\begin{abstract}
(Abstract of paper presented at the International Conference on Nuclear Dnia for Bavic and Applied Sciencess Sante Fe. NM, May 13-17, 1985)

Pairing corrections in particle-hole (exciton) state-density formulas used in precompound nuclear reaction theories are, strictly speaking, dependent on the nuclear excitation energy $U$ and the exciton number $n$. A general formula for $(U, n)$-dependent pairing corrections, based on the the BCS theory, has been derived for exciton state-density formulas for one kind of Fermion. In the present paper, a similar derivation is made for the case of two kinds of Fermions. It is shown that the constant-pairing-energy correction used in standard level-density formulas, such as $U_{0}$, is a limiting case of the present general $(U, n)$-dependent results.
\end{abstract}

- Research sponsoned by the U.S. Department of Energy. 


\section{SIMPLIFIED SPIN CUTOFF FACTORS FOR PARTICLE-HOLE LEVEL DENSITIES IN PRECOMPOUND NUCLEAR REACTION THEORY *}

\section{Y. Fe}

\author{
[Abstract of Nucl. Sci. Eng. (in press)]
}

A logical first step toward incorporating a precompound nuclear reaction theory in the Hauser-Feshbach formalism, widely used for compound reaction cross-section calculations, is to develop unified level density formulas needed for the two parts of the calculation. In the present raper, an advanced formulation of the spin cutoff factors for particle-hole level densities, based on the uniform pairing model, is simplified. This simplified formula, explicitly dependent on the excitation energy and the exciton number, is easy to use for the precompound part of the calculation and is shown to be consistent with the formula used for the Hauser-Feshbach part of the calculation. Differences between the present approach and a previous one are analyzed.

- Research sponsored by U.S. DOE Oftice of Basic Energy Sciences.

\subsection{1}

\section{MULTILEVL ANALXSIS OF THE IOW-ENERGY ${ }^{239} \mathrm{Pu}$ CROSS SECTIONS*}

\section{G. de Saussure R. B. Perez Roger Macklin'}

[Abstract of Trans. Am. Nucl. Soc. 47, 428 (1984)]

Recent and older data on the ${ }^{239} \mathrm{Pu}$ neutron cross se-tions were analyzed using an R-matrix multilevel formalism a:dd the Bayes formula. The resulting parameters describe well the results of recent measurements in the important region below $30 \mathrm{eV}$. The computed cross sections differ from ENDF/B-V mosily in the "valleys" between resonances. The multilevel parameters obviate the need of a not so smooth "ENDF-type file 3" background contribution.

\footnotetext{
- Recearch sponsored by U.S. DOE Division of Reactor Rewearch and Technology.

$t_{1}$ versity of Tennessec, Knoxville, TN.
}

\section{R-MATRIX ANALYSIS OF THE 23"Pu NEUTRON CROSS SECTIONS*}

\author{
G. de Saussure R. B. Perez \\ Roger L. Macklin'
}

\begin{abstract}
(Abstract of paper presented at the International Conference on Nuclear Dala for Basic and Afplier? Sciences, Santa Fe, NM, May 13-17, 1985)
\end{abstract}

The ${ }^{239} \mathrm{Pu}$ neutron cross-section data in the resolved resonance region were analyzed with the R-matrix Bayesian fit program SAMMY. Below $30 \mathrm{eV}$ the cross sections computed with the multilevel parameters are consistent with recent fission and transmission measurements as well as with older capture and alpha measurements. The thermal values are also consistent with the latest evaluation of the thermal parameters.

Above $30 \mathrm{eV}$ no suitable transmission data were available ard only fission cross-section measurements were atislyzed. However, since the analysis conserves the complete covariance matrix, the analysis can be updated by the Bayes method as transmission measurements become available. To date, the analysis of the fission measurements has been completed up to $3 \times 0 \mathrm{eV}$ and an extension to $600 \mathrm{eV}$ is in progress.

"Research sponsored by the U.S. DOE Division of Reactor Research and Technology.

tUniversity of Tennessec, Kiloxville, TN.

1.43

\section{MULTILEVEL ANALYSIS OF THE LOW ENERGY "Pu CROSS SECTIONS*}

\author{
R. B. Perez \\ C. de Sameure \\ N. M. Larsoat \\ Roger Mackliof
}

[Abstract of Nucl. Sct. Eng. (in preas)]

Several sets of ${ }^{239} \mathrm{Pu}$ neutron cross-section data have been analyzed with the $R$-matrix Bayesian program SAMMY. The cross sections computed with the multilevel parameters are consistent with recent fission and transmission measurements, as well as with older capture and alpha measurements. The Reich-Moore type Rmatrix resonance parameters were converted into 
equivalent Adler-Adler type parameters using the computer program POLLA.

* Research sponsored by U.S. DOE Division of Reactor Research and Technology.

tComputing and Telocommunications Division.

tUniversity of Tennessee, Knoxville, TN.

\subsection{4}

\section{REPORT TO THE ${ }^{23}$ U DISCREPANCY TASK FORCE ON SIOB FTTS TO THE ORNL, CBNM, AND JAERI TRANSMISSION DATA*}

\section{K. Obee}

[Abstract of ORNL/TM-9023 (ENDF-338), May 1984]

The computer code SIOB has been used to obtain least-squares simultaneous-sample shape fits to the recent ${ }^{238} \mathrm{U}$ transmission data of ORNL, CBNM, and JAERI over the energy regions 1460 to $1820 \mathrm{eV}, 2470$ to $2740 \mathrm{eV}$, and 3820 to $4000 \mathrm{eV}$. The fits indicate that much of the systematic discrepancy in the published neutron widths from these data arose in the data analysis procedure. Except for the 3820 - to 4000-eV JAERI data, the systematic differences in the resulting neutron widths from the present analyses of the three measurements with no background corrections is less than 2 to $4 \%$. The neutron widths are larger than those contained in any existing evaluation. These fits were performed as part of the work for the NEANDC ad hoc ${ }^{238} \mathrm{U}$ Discrepancy Task Force.

- Research sponsored by U.S. DOE OfTice of Basic Energy Sciences.

1.45

\section{RESOLVED RESONANCE PARAMETERS FOR ${ }^{23:} \mathrm{U}$ FROM 1 TO $10 \mathrm{keV}$ *}

\section{K. Oben}

[Abatract of Nucl. Scl. Eng. (in preas)]

Neutron widths for 676 resonances from 0.9 to $10.0 \mathrm{keV}$ are reported from a consistent leastsquares simultaneous-sample shape analysis of the
Oak Ridge Electron Linear Accelerator (ORELA) 150 -m four-sample ${ }^{238} \mathrm{U}$ transmission data. The neutron widths from 0.9 to $6.0 \mathrm{keV}$ from this improved analysis are meant to supersede previously published values. These resonances give an $s$-wave strength function of about $0.94 \times 10^{-4}$ from 0 to $10 \mathrm{keV}$.

-Research sponsored by U.S. DOE OTice of Besic Energy Sciences.

1.46

\section{STATUS OF THE PARAMETERS OF THE 1.15-keV RESONANCE OF "Fe*}

\author{
F. G. Perey
}

(Abstract of paper presented at the International Conference on Nuctear Data for Basic and Applied Sciences, Santa Fe, NM, May 13-17, 1985)

At the conference in Antwerp, concerns were expressed about large discrepancies in the parameters of the $1.15-\mathrm{keV}$ resonance of ${ }^{56} \mathrm{Fe}$ since they are needed to high arcuracies in some reactor calculations. The Nuclear Energy Agency Nuclear Data Committee organized a task force under the chairmanship of F. G. Perey to coordinate work at CBNM, Harwell, JAERI, and ORNL in order to try to resolve the discrepancies and make recommendations. The 1.15-keV resonance has a value of $\Gamma_{n}$ about 10 times smaller than the value of $\Gamma_{\boldsymbol{\gamma}}$. Therefore, information about these parameters, in particular the capture area of the resonance, can be obtained independently from transmission and capture experiments. At the time of the Antwerp meeting, these two types of experiments appeared to give systematically different values. New transmission and capture experiments were planned at most laboratories using new or wellestablished techniques. Although the work of the task force is not finished (in particular, what may have gone wrong in some of the older experiments is not established and may never be), all transmission experiments completed yield the same value of the resonance parameters, within small uncertainties, independent of the experimental techniques and methods of analysis 
used. Most capture experiments have not been completed. However, at ORNL a new capture experiment using the same type of detector used in an older experiment yields results that are consistent with the transmission results and disagrees with the older capture results. The results of the Task Force, as of the time of the meeting, will be discussed.

-Research sponsored by U.S. DOE Oflice of Basic Energy Sciences.

\subsection{7}

\section{THE NEUTRON CROSS SECTION STANDARDS EVALUATIONS FOR ENDF/B-VI*}
A. U. Carkent
W. P. Poenitz
G. M. Hales
R. W. Peelle

(Abstract of paper presented at the Advisury Group Meeting on Nuclear Standard Reference Data, Central Bureau for Nuclear Measurements, Geel, Belgium. November 12-18, 1984; also presented at the International Conference on Nuclear Data for Basic and Applied Sciences, Santa Fe, NM. May 13- 17, 1985)

The neutron cross section standards are now being evaluated as the initial phase in the development of the new ENDF/B-VI file. These standards evaluations are following a somewhat different process compared with that used for earlier versions of ENDF. The primary effort is concentrated on a simultaneous evaluation using a generalized least squares program, R-matrix evaluations, and a procedure for combining the results of these evaluations. The ENDF/B-VI standards evaluation procedure is outlined, and preliminary simultaneous evaluation and $\mathbf{R}$ matrix results are presented.

\footnotetext{
- Rescarch sponsored by U.S. DOE Division of Reactor Rescarch and Technology.

${ }^{t}$ National Bureau of Standards, Gaithersburg. MD.

FArgonne National Laboratory, Argonne-Weat, Idaho Falls, ID.

SLa Alamos National Laboratory, Los Alama, NM
}

1.48

\section{REPORTS ON NUCLEAR DATA \\ ACTIVITIES FOR THE DOE \\ NUCLEAR DATA CUMMITTEE*}

\author{
D. C. Larson
}

[Abstract of BNL-NCS-34291, p. 138 (May 1984) and BNL-NCS-36272, p. 125 (May 1985)]

These two reports were prepared for the DOE Nuclear Data Committee and cover work performed at ORNL since January 1983 in areas of nuclear data of relevance to the U.S. applied nuclear energy program. The reports were mostly generated through a review of abstracts of work completed to the point of being subjected to some form of publication in the open literature, formal ORNL reports, ORNL technical memoranda, progress reports, or presentation at technical conferences. As much as possible we have reproduced the complete abstracts of the original publications with only minor editing In a few cases progress reports were written specifically for this publication. The editor has selected the materials to be included in this report on the basis of perceived interests of DOE Nuclear Data Committee members and cannot claim completeness.

-Reacarch sponsored by U.S. DOE OfTice of Basic Energy Sciences. 
1.49

\section{OFFICE OF BASIC ENERGY SCIENCES PROGRAM TO MEET \\ HIGH PRIORTY NUCLEAR DATA \\ NEEDS OF THE OFFICE OF \\ FUSION ENERGY: SUMMARY \\ OF RESULTS 1976-1983*}

$$
\text { D. C. Larsoa R. C. Henight }
$$

(Abstract of ORNL/TM-9335, September 1984)

Experimental data acquired under the BES program to meet Fusion Data Needs from its inception (1975) to 1983 are summarized by element, reaction, energy, and contributing laboratory. Cases where the data have been incorporated in ENDF evaluations are noted. Work in nuclear model code development under this program is summarized.

- Research sponsored by U.S. DOE Offree of Basic Energy Sciences.

tLawrence Livermore National Laboratory, Livermore, CA.

\section{IMPROVING NEUTRON REACTION DATA THROUGH LINAC-BASED FLIGHT-TTME SPECTROMETRY*}

\section{R. W. Peelle}

[Abstract of Trans. Am. Nucl. Soc. 49, 206 (1985)]

Pulsed neutron facilities based on electron linear accelerators (linacs) allow measurements of neutron cross sections from $E_{n} \sim 0.01 \mathrm{eV}$ to $E_{n}$ $>20 \mathrm{MeV}$. The text outlines the capabilities of such facilities and their role in upgrading the neutron cross-section data base using the Oak Ridge Electron Linear Accelerator (ORELA) as the prime example.

The capabilities of ORELA to provide improved data are discussed for various types of reactions. With help from ORELA, expected data needs have been met for many nuclides and reactions. For others, interim data have been provided and techniques developed that can be used 10 meet additional needs. Total, capture, and fission cross sections, as well as photon and neutron yield and neutron multiplicity measurements, are discussed with respect to the status of present data and the prospects for the future.

Though neutron spectrometry based on electron linacs is well developed, the now-existing accelerators have not been fully exploited and some of the most important measurements are yet to be performed. Future experiments will concentrate on areas in which instruments or analysis techniques are new and accuracy requirements are most stringent, or where new physics or technology interests arise. Users of neutron cross sections face unnecessary ambiguities when available cross sections are insufficiently accurate for the tasks at hand; these cross-section sets must b: grounded in experiment, though theory sho ild play a great role. For a large dynamic range of neutron energy and for many reaction t:pes, the electron linear accelerator provides the source of choice for experiments to improve the neutron data base.

"Research sponsored by U.S. DOE OfTice of Bacic Enctsy Sciences.

1.51

\section{SUMMARY TAL COVERING APPLICATION-ORIENTED SESSIONS*}

\author{
R. W. Peile
}

(Abatract of paper presented at the International Cooference on Nuclear Dats for Basic and Applied Sciencea, Santa Fe, NM, May 13-17, 1985)

- Conference contributions are reviewed relative to the applications of neutron cross sections to technology. The field of neutron cross section research is described as well balanced with respect to the various categories of research ranging from basic science to engineering, and the detailed data being obtained are matched to the needs and capabilities of the technology. "Victories" have been won in the last several years in the areas of nonfuel actinide cross sections, 
fusion charged particle reactions, the $\mathrm{Li}-7$ breakup reaction, the number of neutrons emitted per fission, short-term decay heat from fission products, the shape of the fission neutron spectrum, and neutron scattering differential cross sections. Several research approaches and shifts of research concentration are welcomed as indicating favorable trends. Challenges for the future are identified in the areas of kerma data for neutron energies above $10 \mathrm{MeV}$, helium production from neutrons reacting with boron-10, the acquisition of fully satisfactory data for the resonance and thermal regions of fuel nuclides, and satisfying tightened accuracy targets for the most important cross sections. It is concluded that while the normal-accuracy needs of various applications are being met, neutron cross-section scientists throughout the world need to focus attention on meeting the most important and demanding challenges.

- Research sponsored by U.S. DOE Division of Reactor Research and Technolozy.

\section{Techniques and Facilities}

1.52

\section{UNCERTAINTY PROPAGATION FROM RAW DATA TO FINAL RESULTS*}

\section{N. M. Larson'}

(Abstract of paper prasented at the International Conference on Nuclear Data for Basic and Applied Sciences, Santa Fe, NM, May 13-17, 1985)

Reduction of data from raw numbers (counts per channel) to physically meaningful quantities (such as cross sections) is in itself a complicated procedure. Propagation of experimental uncertainties through that reduction process has sometimes been perceived as even more difficult, if not impossible. At the Oak Ridge Electron Linear Accelerator, a computer code ALEX has been developed to assist in the propagation process. The purpose of ALEX is to carefully and correctly propagate all experimental uncertainties through the entire reduction procedure, yielding the complete covariance matrix for the reduced data, while requiring little additional input from the experimentalist beyond that which is needed for the data reduction itself. The theoretical method used in ALEX is described, with emphasis on transmission measurements. Application to the natural iron and natural nickel measurements of $D$. C. Larson is shown.

\footnotetext{
- Rescarch sponsored by U.S. DOE Office of Basic Energy Sciences and Division of Reactor Revearch and Technology.

'Computing and Telecommunicatione Division.
}

1.53

\section{UPDATED USERS' GUIDE FOR SAMMY: MULTILEVEL R-MATRXX FTTS TO NEUTRON DATA USING BAYES' EQUATTONS*}

\author{
N. M. Larsoa' ${ }^{\dagger}$ \\ (Abstrz:", of ORNL/TM-9179, August 1984 and \\ ORNL/TM-4179/RI, April 1985) \\ ORNL/TM-9179:
}

In 1980 the multilevel multichannel R-matrix code SAMMY was released for use in analysis of neutron data at the Oak Ridge Electron Linear Accelerator. Since that time, SAMMY has undergone significant modifications: (1) Userfriendly options have been incorporated to streamline common operations and to protect a run from common user errors. (2) The ReichMoore formalism has been extended to include an optional logarithmic parameterization of the external R-matrix, for which any or all pasameters may be varied. (3) The ability 10 vary sample thickness, effective temperature, matching radius, a.d/or resolution-broadening parameters has been incorporated. (4) To avoid loss of information (i.e., computer round-off errors) between runs, the "covariance file" now includes precise values for all variables. (5) Unused but correlated variables may be included in the analysis.

Because of these and earlier changes, the 1980 SAMMY manual is now hopelessly 
obsolete. This report is intended to be complete documentation for the current version of SAMMY. Its publication in looseleaf form will permit updates to the manual to be made concurrently with updates to the code itself, thus eliminating most of the time lag between update and documentation.

\section{ORNL/TM-9179/R1:}

In August of 1984 the users' guide for version P of the multilevel multichannel R-matrix code SAMMY was published. Recently, major changes within SAMMY have led to the creation of version $\mathrm{O}$, which is documented in this report. Among these changes are: (1) an alternative matrix-manipulation method for use in certain special cases; (2) division of theoretical cross-section generation and broadening operations into separate segments of the code; (3) an option to use the multilevel Breit-Wigner approximation to generate theoretical cross sections; (4) new input options; (5) renaming all temporary files as SAM??.DAT; (6) more sophisticated use of temporary files to maximize the number of data points that may be analyzed in a single run; and (7) significant internal restructuring of the cude in preparation for changes described here and for planned future changes.

-Research sponsored by U.S. DOE Office of Basic Encrey Sciences.

Computing and Telecommunications Division.

1.54

\section{ANALYSIS OF NEUTRON DATA IN THE RESONANCE REGION VIA THE COMPUTER CODE SAMMY}

\author{
N. M. Larsoa ${ }^{\prime}$
}

(Abstract of paper presented at the International Conference on Nuciear Data for Basic and Applied Sciences, Santa Fe, NM, May 13-17, 1985)

Analysis and evaluation of resonance neutron cross-section data require sophisticated computational procedures. Such procedures have been implemented in a state-of-the-art computer code SAMMY, developed at the Oak Ridge Flectron Linear Accelerator at Oak Ridge National Laboratory (N. M. Larson, Updated
Users' Guide for SAMMY, ORNL/TM-9179, 1984). Notable features of SAMMY include: (1) Use of Bayes' equations to determine 'best" values of parameters. This permits sequential analysis of data sets (or subsets) while giving the same results that a simultaneous analysis would give; examples will be shown. (2) Choice of multilevel Breit-Wigner formulation or ReichMoore approximation to R-matrix. (3) Logarithmic parameterization of the external R-matrix. (4) Choice of transmission, total cross section, scattering, fission, capture, or absorption cross sections; examples of several will be shown. (5) Several options for Doppler and/or resolution broadening. (6) Inclusion of off-diagonal data correlations if desired. (7) Analysis of different data types simultaneously. (8) Inclusion of channel radius, broadening parameters, sample thickness, and/or parameters of external R-matrix in the fitting procedure. (9) Dynamic allocation of array storage space. Thus, there are no rigid limits on any of the "numbers" - number of resonances, number of varied parameters, number of data points, etc. The only rigid limit is the size of the computer. (10) Frequent $1 / O$ to and from temporary files to maximize the size of a job which can be run. (11) Thorough documentation in the form of a complete user's guide. (12) Versions currently available on Decsystem-10 (PDP-10), IBM-360, and VAX computers. Not all features available on all machines.

- Research sponsored by U.S. DOE Office of Benic Enerty Sciencea and Division of Reactor Research and Technolosy.

${ }^{t}$ Computing and Telecommunications Division.

\subsection{5}

USER'S GUIDE FOR ALEX: UNCERTAINTY PROPAGATION FROM RAW DATA TO FINAL RESULTS FOR ORELA TRANSMISSION MEASUREMENTS*

\section{N. M. Larson't}

[Abstract of ORNL/TM-8676 (ENDF-332), February 1984]

This report describes a computer code (ALEX) developed to assist in Analysis of EXperimental data at the Oak Ridge Electron 
Linear Accelerator (ORELA). Reduction of data from raw numbers (counts per channel) to physically menningful quantities (such as cross sections) is in itself a complicated procedure; propigation of experimental uncertainties through that reduction procedure has in the process been viewed as even more difficult - if not impossible. The purpose of the code ALEX is to correctly propagate all experimental uncertainties through the entire reduction procedure, yieiding the complete covariance matrix for the reduced data, while requiring little additional input from the experimentalist beyond that which is required for the data reduction itself. This report describes ALEX in detail, with special attention given to the case of transmission measurements (the code itself is applicable, with few changes, to a.ny type of data). Application to the natural iron neasurement of D. C. Larson et al. is describe' in some detail.

-Research sponsered by U.S. DOE Office of Basic Eners] Sciences.

'Computing and Telecommunieations Division.

\subsection{6}

\section{MAJORITY LOGIC NE-110 DETECTORS FOR keV NEUTRONS*}

\author{
N. W. Hill ${ }^{t}$ \\ J. A. Harrey \\ D. J. Horen ${ }^{t}$ \\ G. L. Morgans
}

\section{R. R. Winters'}

(Abstract of paper presented at IEEE 1984 Nuclear Science Symposium, Orlando, FL, October 31-November 2, 1984)

Proton recoil scintillation detectors, whose efficiencies are reproducible and stable, have been developed for neutrons in the energy range from $5 \mathrm{keV}$ to $1 \mathrm{MeV}$. One of the detectors is a $6 \times$ $6 \times 2 \mathrm{~cm}$ scintillator with a RCA 8850 photomultiplier on three of its faces. The second one is a $7.6-\mathrm{cm}$-diam $\times 4.3-\mathrm{cm}$-thick cylindrical detector with two phototubes. The discriminator for each photomultiplier is biased well below the single photoelectron level; majority logic eliminates phototube noise and requires one or more photoelectrons in at least two tubes. Pulse height measurements of the summed signals for both detectors have been made from $5 \mathrm{keV}$ to 350 keV using both a continuous energy neutron beam and $\mathrm{Fe}$ "window" neutrons. The experimental results were compared with Monte Carlo calculations to obtain absolute neutron detection efficiencies for these detectors. These detectors have been used recently for neutron inelastic and differential elastic-scattering measurements.

\footnotetext{
-Research sponsored by US. DOE OTIce of High Energy and Nuckear Physias.

Instrumentation and Controls Division.

Physics Division.

fLos Alamos National Laboratory, Los Alamos, NM.

Deaison University, Granville, OH.
}

\subsection{7}

\section{AN NE-213-SCINTILLATOR-BASED NEUTRON DETECTION SYSTEM FOR DIAGNOSTIC MEASUREMENTS OF ENERGY SPECTRA FOR NEUTRONS HAVING ENERGIES $\geqslant 0.8 \mathrm{MeV}$ CREATED DURING PLASMA OPERATIONS AT THE PRINCETON TOKAMAK FISION TEST REACTOR*}
J. K. Dickens
N. W. Hill
F. S. Hoot
J. W. McConents
R. R. Speacer
F. Y. Tsang ${ }^{+}$

(Abstract of ORNL/TM-956I. August 1985)

A system for making diagnostic measurements of the energy spectra of $\geqslant 0.8-\mathrm{MeV}$ neutrons produced during plasma operations of the Princeton Tokamak Fusion Test Reactor (TFTR) has been fabricated and tested and is presently in operation in the TFTR Test Cell Basement. The system consists of two separate detectors, each made up of cells containing liquid NE-213 scintillator attached permanently to RCA-8850 photomultiplier tubes. Pulses obtained from each photomultiplier system are amplified and electronically analyzed to identify and separate those pulses due to neutron-induced events in the detector from those due to photon-induced events in the detector. Signals from each detector are routed to two separate analog-to-digital converters, and the resulting digitized 
information, representing (1) the raw neutronspectrum data and (2) the raw photon-spectrum data, are transmitted to the CICADA dataacquisition computer system of the TFTR. Software programs have been installed on the CICADA system to analyze the raw data to provide moderate-resolution re-creations of the energy spectrum of the neutron and photon fluences incident on the detector during the operation of the TFTR. A complete description of, as well as the operation of, the hardware and software is given in this report.

- Research sponsored by US. DOE OTice of Basic Enersy Sciences and wort partially performed by Princeton Plasma Physica Laboratory, Primotion, NJ.

IInstrumentation and Controls Division.

IPribceton Pla a Physics Labontory, Princeton, NJ.

Fphysica Division.

${ }^{+}$Idabo National Enginecring Laboratory, Idabo Falls, ID.

1.58

\section{A PRELIMINARY STUDY OF POSSIBLE ORELA REPLACEMENT OPTIONS*}

\section{K. Olsen J. A. Martin D. J. Horea'}

(Abatract of ORNL/TM-8669, June 1984)

Based on two conceptual design studies performed by the LANL Accelerator Technology Division, the possibilities in terms of accelerator systems for replacing ORELA with a more intense Maxwellian-type continuous-energy neutron source are summarized and discussed. The neutron intensities from ORELA are compared with those from existing or potential accelerator systems used for cross-section and condensed-matter studies. The best replacement options seem to involve a spallation source from 20) - to $400-\mathrm{MeV}$ protons on an ORELA-like target. Pulsing and intensity desiderata with such a source are discussed which correspond to a sfertrum-averaged 100-fold improved figure of merit over ORELA for TOF measurements with only a tenfold increased source strength. Existing accelerator designs seem to be inadequate for such a source. Consequently, two conceptual designs were developed for this study by the LANL Accelerator Technology Division. The first conceptual design is for a $200-\mathrm{MeV}$ large linac capable of accelerating $1.3 \mathrm{~A}$ during a mactopulse; this linac standing alone could serve as an ORELA replacement source. The second conceptual design is for a much smaller $250-\mathrm{MeV}$ PIGMI linac with a 2.8-mA macropulse current which feeds a proton accumulator ring and bunch-compressor transpert line. This linacring-compressor (LIRIC) option would give a more cost-effective neutron source for crosssection measurements, whereas the large linac, or a modified version of it, would give a much simpler system more suitable for expansion. In particular, both conceptual designs would incorporate the present ORELA building anu would provide approximately 100 -fold improved neutron sources over ORELA for cross-section measurements. The total estimated cost of the LIRIC system would be \$43M (1984), whereas the cost of the large linac would be about a factor of two more.

- Research sponsored by U.S. DOE Oftice of Besic Eoergy Sciences.

'Physics Division.

\section{CalCULATiJNS PERTAINING TO THE DESIGN OF A PREBUNCHER FOR A 150-MeV ELECTRON LINEAR ACCELERATOR III. COMPARISONS WITH EXPERIMENTAL DATA*}

\author{
R. G. Alariller, Jr. F. S. Alamiller \\ T. A. Lents"
}

(Abstract of ORNL/TM-9375, November 1984; aleo Pant Acceler. 17, 215 (1985); also peper presented at Southeastern Section Meeting of the American Phynical Society, Memphis, TN, October 24-27, 1984]

In a previous paper a ballistic model that included space-charge effects was described and calculated results were presented of the extent to which a current pulse of electrons ( $\sim 150 \mathrm{keV}$ kinetic energy, $\sim 1 \mu \mathrm{C}$ of charge, and $\sim 15$ nsec 
full width at half maximum) could be bunched, i.e, reduced in width without loss of charge, by passing it through a series of gaps on which time-dependent voltages are applied. A prebuncher system similar to the one considered previously has now been constructed, and experimental data on the current at the end of the prebrincher as a function of time have been obtained. Here the calculated current as a function of time, obtained using the model developed previously, is compared with the experimental data. The calculated and experimental data are in substantial agreement for a variety of electron-beam and voltage-gap conditions.

- Research sponsored by U.S. DOE Office of Basic Energy Sciences.

'Inatrumentation and Controls Division.

\subsection{0}

\section{ORELA FLIGHT PATH I: DETERMINATIONS OF ITS EFFECTIVE LENGTH VS ENERGY, EXPERIMENTAL ENERGIES, AND ENERGY RESOLUTION FUNCTION AND THEIR UNCERTAINTIES*}

\section{C. Larson N. M. Larsont}

\section{J. A. Harrey}

(Abatract of ORNL/TM-8880, June 1984)

Flight path 1 at ORELA is nominally $200 \mathrm{~m}$ in length and has been extensively used for neutron transmission and scattering measurements. Due to moderation effects in the neutron-producing target and to the finite thickness of the neutron detector, the effective flight-path length is a function of neutron energy. In this report, we determine the effective length as a function of energy, its uncertainty, and time-of-flight energies and their uncertainties. Finally, we determine the resolution function and its uncertainty and compare the width of this resolution function with an experimental determination of this quantity.

- Recearch sponsored by U.S. DOE OTIce of Basic Ezerzy Sciences.

${ }^{t}$ Comprting and Telecommunications Division.

\subsection{1}

\section{LASER MEASUREMINTS OF DISTANCES FROM THE ORELA NEUTRON TARGET TO EXPERIMENT STATIONS ALONG FUGHT DATHS 1 AND 6*}
D. C. Larson
N. M. Larsoa $t$
J. A. Harrey
F. G. Perey
D. E. Piercé
R. H. Sealss

(Abstract of ORNL/TM-9097. March 1985)

Flight-path lengths have been measured by laser techniques for the 200-,80, and 18-m stations along flight path 1 , and for the 5-, 20-, 40-, and $150-\mathrm{m}$ stations along flight path 6 at the Oak Ridge Electron Linear Accelerator (ORELA). In each case the distance evaluated from the measurements is the slope distance from the center of the neutron-producing target to a position along the beam path, directly above a suitable benchmark at the experiment station. A total of 25 laser measurements were performed between the various stations. These data, along with appropriate uncertainties, were combined using Bayes' method. From this analysis we obtained the desired night-path lengths, which typically have uncertainties less than $1.5 \mathrm{~mm}$. The measurement technique, uncertainties, analysis method, and results are documented in detail in this report.

- Research sponsored by U.S. DOE OfTice of Basic Energy Sciences.

${ }^{t}$ Computing and Telocommunications Division.

SPlant and Equipment Division. 
Section 2

SYSTEMS ANALYSIS AND SHIELDING 


\title{
2.0. INTRODUCTION
}

\author{
D. G. Cacuci
}

During this reporting period, the Systems Analysis and Shielding Section has performed work in four major categories: (1) radiation transport methods and data development for shielding applications, including development and applications of advanced transport codes, generation and evaluation of nuclear data libraries, and experimental benchmarking and confirmation of shield designs; (2) methods and data development for assessing performance of several types of operating nuclear reactors, and for design of both advanced commercial reactors and a special-purpose high flux reactor; (3) studies of nuclear weapons effects; and (4) mathematical and applied physics, including transport theory, classical and quantum kinetics and hydrodynamics, and nonlinear operator equations that can display bifurcations and chaotic behavior.

\section{Radiation Transport Methods and Data}

The development of radiation transport codes was continued for both Monte Carlo and discrete ordinates methods. Thus, a capability to construct complex geometries using modular arrays was implemented into the Monte Carlo transport code MORSE. The general attributes and capabilities of several related Monte Carlo codes were evaluated, and new biasing schemes were studied. Also, we organized a workshop on the theory and use of MORSE; this workshop was well attended by domestic and foreign participants representing a wide range of interests.

The development of the three-dimensional discrete ordinates code TORT has progressed significantly, including incorporation of several auxiliary codes. In particular, the development and implementation of a $k$-calculation capability will enhance the applicability of TORT to a significantly wider range of problems.

Our two-dimensional transport code DOT - used world wide for shielding applications - has also undergone further development by implementation of a linear nodal method. This method, as implemented into DOT, was shown to be superior to the weighted difference methods for several practical shielding problems.

Work has continued for both a DOE program and a Defense Nuclear Agency (DNA) program to construct new multigroup cross-section libraries based entirely on ENDF/B-V nuclear data. These libraries will replace cross-section data derived from earlier versions of ENDF.

\section{Radiation Shielding Applications}

A significant effort was devoted to the analysis of shields for DOE-supported advanced reactor designs, including the high-temperature gas-cooled reactor (HTGR) and innovative liquid-metalcooled reactors (LMR). It is important to note here that DCE has categorized work for the LMR 
and, more recently, the HTGR programs as "Applied Technology," and thus explicit references to much of our work related to these programs cannot be included in this report.

For the HTGR, the second phase of a two-part experiment at the ORNL Tower Shielding Facility was performed and analyzed in order to verify the significant neutron streaming that was predicted in the bottom reflect sr and core support region. The analytic predictions yere largely confirmed; this fact demonstraied the need to redesign the lower reflector shield in the HTGR. Radiation analyses were also performed for a modular pebble bed HTGR design. This geometrically smaller design, which is fromoted by "inherently safe" and "shop fabrication" considerations, places an increased demand on accurate shielding analyses.

The LMR shielding support focused on scoping analyses and trade-off studies for the modular reactor designs proposed by General Electric and Rockwell International. As with the HTGR program, the move to smaller designs has placed new emphasis on shielding effectiveness and the assessment of reactor componer.t exposures. An experiment intended to benchmark new shielding materials for LMRs has been initiated at the ORNL Tower Shielding Facility (TSF). This experiment represents the beginning of a major multi-year program of experimental shielding research in cooperation with the Japanese LMR program.

A significant effort was alsi) devoted in support of the joint U.S.-Japan program to reassess the doses received by survivors of Hiroshima and Nagasaki. Within this effort, the newly developed three-dimensional code TORT, logether with several auxiliary service codes, was used to calculate the doses received by survivors located inside large concrete buildings. The initial results indicate that the survivors inside these buildings received doses which range from below to significantly above the current LD-50 limit (the dose level at which $50 \%$ of the victims are expected to die), and hence provide valuable data for the possible revision of this important limit. The results calculated by TORT agreed well with an integral experiment performed at the TSF for the purpose of validating such calculations. A related TSF activity was devoted to the accurate measurement of the leakage spectra from a critical mockup of the Hiroshima weapon. (Additional research performed by our division in support of the joint U.S.-Japan Dose Reassessment Program is reported below under the heading "Studies of Nuclear Weapons Effects.")

\section{Reactor Analyses}

\section{Design of a New High Flux Reactor for the Proposed \\ Center for Neutron Research}

We have performed exten:ive research to design a high flux reactor (HFR) for the proposed Cente, for Neutron Research (CNR). A reference design was completed and presented at the International Workshop on an Advanced Steady State Neutron Facility (National Bureau of Standards, December 16-17, 1985). According to the Eastman-Seitz Committee of the National Academy of Sciences, there is a need to build a facility capable of producing a thermal-neutron flux peak larger than $5 \times 10^{15} / \mathrm{cm}^{2} \cdot \mathrm{sec}$ for use as a scattering source for research in solid state physics, biology, material scien ies, basic nuclear physics, etc. None of the existing steady sources of neutrons in the world produce such a neutron flux. Other desired characteristics of the propoed HFR are that it be capable of operating continuously for a period of at least two weeks, produce isotopes, and irradiate materials. This multiple use of this reactor, together with the requirement of the high thermal flux, constitutes a major challenge for designers.

The design of this HFR re.ies crucially on the processes of slowing down and diffusion of ne' trons in the moderator. To undirstand these processes, we have developed an analytical solution for 
an idealized HFR by parametrizing the design, highlighting the most important variables, and comparing the reference design with other alternatives. This analytical solution has shown that the flux efficiency (i.e., the ratio of thermal-neutron flux leakage to the power) can be maximized provided that (1) the volume of the core and the diffusion lengths of thermal neutrons in the core and reflector are minimized and (2) the escape of neutrons from the core maximized.

Following extensive simulations performed with both analytical and numerical models, we have proposed a near-optimum reference HFR design whose efficiency is $29 \%$ larger than the world's best facility presently in operation (the ILL reactor). The projected unperturbed peak thermal flux for this design is 5 to 10 times $10^{15} / \mathrm{cm}^{2} \cdot \sec$ for a total power of 135 to $270 \mathrm{MW}$.

\section{Criticality Safety Studies}

The ${ }^{252} \mathrm{Cf}$ method, which has been used extensively to measure reactivities in a large variety of reactor systems ranging from a fast reactor mockup to thermal homogeneous systems, is now being applied to arrays of cans containing fissile solutions and pieces of uranium metal. The geometrical complications of such arrays preclude the use of customary interpretative models based on point reactor or bare reactor eigenfunction expansions. Thus, we have developed models based on a phenomenological multipoint reactor formalism and on a postulated eigenfunction expansion, respectively. In addition, we have calculated stochastic observables with a lumped two-point and a continuous two-mode reactor model.

Our research has led to an interpretative model that can be used for any system (discrete or continuous, symmetric or unsymmetric, uniform or non-uniform) that has a discrete adjoint kinetic eigenfunction set. The suitability of the proposed model to interpret ${ }^{252} \mathrm{Cf}$ source noise reactivity measurements in complicated geometries has been validated against experiments for the simplified case of two interacting cans of fissile material.

\section{Nonlinear Dynamics and Stability of Boiling Water Reactors}

A phenomenological model has been developed to simulate ihe qualitative behavior of boiling water reactors (BWRs) in the nonlinear regime under deterministic and stochastic excitations. After the linear stability threshold is crossed, limit cycle oscillations appear caused by interactions between two unstable equilibrium points and the phase-space trajectories. This limit cycle becomes unstable when the feedback gain exceeds a certain critical value. Subsequent limit cycle instabilities produce a cascade of period-doubling bifurcations that leads to aperiodic pulsed behavior.

Under stochastic excitations, BWRs exhibit a single characteristic resonance, at $-0.5 \mathrm{~Hz}$, in the linear regime. By contrast, this work has shown that harmonics of this characteristic frequency appear in the nonlinear regime. Furthermore, this work has also demonstrated that the amplitudes of the limit cycle oscillations do not depend on the variance of the stochastic excitation and remain bounded at all times.

A physical model of nonlinear BWR dyna.. - was also been developed and employed to calculate the amplitude of limit cycle oscillations a in their effects on fuel integrity over a wide range of operating conditions in the Vermont Yankee reactor. These calculations have confirmed that, beyond the threshold for linear stability, the reactor's state variables undergo limit cycle oscillalions. Our work has shown that the amplitudes of these oscillations are very sensitive to changes in operating conditions and are not restricted to small magnitudes as observed in previous stability tests. Consequently, large-amplitude limit cycle oscillations become a possible scenario for BWR operation at low-flow conditions. The effects on fuel integrity of such large-amplitude oscillations 
have been studied in detail. In particular, we have shown that limit cycles that oscillate with frequencies $>0.25 \mathrm{~Hz}$ and reach the high-power safety scram level of $120 \%$ are no: likely to compromise fuel integrity.

The significance of this work has been recognized by the granting of the American Nuclear Society's Mark Mills Award for 1985.

\section{HTGR Metiods Derelopmeat and Calculations}

Under the auspices of the DOE Program for Advanced Converter Reactors, a continuing effort has been direrted to the development and application of improved methods for the analysis of HTGR physics and thermal-hydraulic problems of interest to DOE's industrial contractors. Prior to the end of fiscal year 1984, national program emphasis had been placed on support of a large integrated HTGR design rated at $2240 \mathrm{MW}(\mathrm{tb})$. During this period, coupled neutronic and thermal-hydraulic methods were developed and employed to assess the optimum power density for the large HTGR. Furthermore, the impact of power density on core and reacter vessel size, pumping power requirements, inlet flow orificing patterns and hot channel thermal margins had to be factored into the estimate of capital costs and ease of licensing of these large HTGRs.

Additional work was carried out in direct support of the DOE's prime industrial HTGR contractor, GA Technologies, Inc. A review and evaluation was performed of GA's hexagonal nodal simulator for reactor physics calculations. Theoretical inconsistencies were identified in the coupling model of the GA computer code, and two- and three-dimensional HTGR benchmark calculations were performed employing the DIF3D hexagonal nodal simulator which had originally been developed and employed for fast reactor analysis. The demonstration of successful applications of the in-house modified DIF3D to the HTGR prismatic core has been received with great interest by both GA and the Public Service Company of Colorado, owner/operator of the Fort St. Vrain HTGR.

Depletion perturbation theory (DPT) methods, which were developed and demonstrated in the breeder reactor program work at ORNL, were implemented into the GA one-dimensional diffusion computer code FEVER-M1. GA requested this implementation to allow the use of DPT methods in evaluating optimum axial fuel and poison zoning in advanced HTGRs using prismatic fuel elements. This effort has also led to the theoretical definition and code implementation of the DPT equations for sensitivity coefficients that account for burnup-dependent flux shielding factors. Such sensitivity coefficients had not been encountered in previous DPT work at ORNL since fast reactors are not subject to flux depressions as severe as those affecting the fuel and burnable poison nuclidic cross sections in a HTGR.

In addition to methods development, detailed reviews were performed of GA's cross-section data and methods used in calculating safety-related reactor physics data. Calculations were also performed for HTGR physics benchmarks comparing ORNL and GA data and methods. The results have identified potential sources of error in GA data processing of uranium-238 and the apparent overprediction of the temperature reactivity defect by GA on the low enriched fuel (LEU) fuel cycle. GA is now seeking additional funding to address these and other deficiencies identified by the ORNL audit. In the same time period, thermal-hydraulic methods were develor ed and benchmarked for simulating shutdown passive heat removal from either prismatic or pebble bed fueled modular HTGRs. Independent studies of cylindrical and annular ires were performed to confirm West German and U.S. industry preliminary calculations of shutdown heat removal under loss of normal cooling accidents. 
At the beginning of fiscal year 1985, the DOE HTGR program switched emphasis from large integrated reactors to smaller modular plants. Since initial interest in the modular HTGR focused on the cylindrical $250-\mathrm{MW}(\mathrm{th})$ pebble reactor using the LEU fuel cycle, reactor physics calculations were performed to assess the temperature coefficient of reactivity, the reactivity effect of water ingress, and the effectiveness of outer reflector control rods for control and shutdown. This effort was augmented by an international project with the Kernforschungsanlage (KFA) Juelich GmbH and the Arbeitsgemeinschaft Versuchs-Reaktor (AVR) GmbH in the Federal Republic of Germany to perform benchmark analysis of AVR reactor physics with both high enriched uranium/thoriun and LEU fuel loadings. With the September 1985 decision by DOE to concentrate on an annular 350-MW(th) prismatic core for the modular HTGR, further work will be realigned to address reactor physics safety and licensing issues for this concept.

\section{Noclear Power Optioas Yiability Stedies}

Under auspices of the DOE-funded Nuclear Power Options Viability Study (NPOVS) performed at ORNL, the commerciai viability and research and development needs have been evaluated for advanced passively safe concepts for the LWR and HTGR. These concepts include the Process Inherent Ultimate Safety (PIUS) PWR propose 1 by ASEA-ATOM in Sweden, the small BWR from General Electric and the DOE-sponsored m:xular HTGR. The cost/benefit of passive safety features were assessed in terms of the perceived enhanced ease of licensing, reduced construction requirements for a non-safety grade balance of plant, and the elimination of systems associated with active engineered safety features. Results indicate that reactors relying on passive safety features may have an advantage in public acceptance but require additional effort to be competitive with coal-fired plants and the best LWR technology.

\section{Andysis of Reactor Operating Data}

We have provided technical assistance to the Nuclear Regulatory Commission (NRC) Office for Analysis and Evaluation of Operational Data in assessing the operational experience derived from the Fort St. Vrain High Temperature Gas-Cooled Keactor (HTGR). This technical assistance included screening of all Fort St. Vrain operating data sources and licensing material and an in-depth evaluation of the partial failure-to-scram event of June 1984. Our ORNL monthly and terhnical reports have contributed to the licensing evaluatinns and actions issued by the NRC Office of Nuclear Reactor Regulation regarding the Fort St. Vrain restart and continued operation. Results of the analysis of operating experience from Fort St. Vrain were also reported to the International Atomic Energy Agency (IAEA) meeting on gas-cooled reactor safety held in Oak Ridge in May 1985. The dissemination of the ORNL evaluations to other NRC offices has led to additional requests for assistance by those offices.

\section{Studies of Nuclear Weapons Efrects}

\section{Reassesement of Atomic Bomb Radiation Docimetry in Hiroshima and Nagasaki}

In addition to the experimental and calculational results reported in the foregoing under the heading "Radiation Shieldiny Applications," our division's participation in the joint U.S.-Japan Dose Reassessment Program has centered around recalculation of the air/ground radiation environment using updated air and ground compositions, re-analysis of the sulfur aclivation in electric pole insulators, and sensitivity/uncertainty analysis of the tissue kerma for various locations at the air/ground interface in the Hiroshima and Nagasaki environments. 
The re-analysis of the sulfur activation in electric pole insulators at Hiroshima became necessary once the updated air and ground compositions and additional information on the insulators' locations became available. Analysis of this sulfur activation is needed for improving the estimate of the bomb yield of the Little Boy Weapon.

Sensitivities of kermas to neutron and gamma rediation at various ground locations in both Hiroshima and Nagasaki have been generated using the latest environmental, geographical, and weapons information. The uncertainty analysis has proceeded by combining these sensitivities with covariances calculated for both the Hiroshima and Nagasaki environments. Final work in this area will lead to a systematic overall uncertainty analysis for the two cities. The results of this sensitivity and uncertainty analysis effort will be documented in a joint U.S.-Japan report.

\section{Wort in Sepport of the Federal Emergency Mamagement Ageacy (FEMA)}

In 1983, a project sponsored by the Federal Emergency Management Agency (FEMA) and titled "Improvement and Updating of Radiological Defense Handbooks and Procedures" was transferred from the ORNL Energy Division into the Engineering Physics and Mathematics Division. One of the previous handbooks resulting from this project is Radialion Safety in Sheleers, which has had over 100,000 copies printed and distributed so far. This handbook was reviewed in The Health Physics Society's Newsletter, September 1984.

During the past year, the Radiological Defense Handbook was rewritten and renamed Radiological Protection Manual. This book presents a broad spectrum of nuclear science at a high-school level, including basic physics of nuclear radiation, principles of radiation detection, a review of the types and operating principles of radiological instruments, biological effects of nuclear radiation, reviews of possible emergency situations that can produce radiological hazards, nuclear weapon effects, principles of radiation exposure monitoring and control, principles of radiation protection, and radiological decontamination. Within the next few years, the Radiation Protection Manual will replace the widely distributed copies of the old handbook.

Also within the FEMA program, a new method has been developed for forecasting radiation exposure from multi-aged fallout from nuclear weapons. The method does not require knowledge of the yields or detonation times of the contributing weapons and is relatively insensitive (compared with older methods) to superposition of fallout of different ages or to the actual decay rate of the fallout. A paper describing this method has been accepted for publication in Health Physics, and nomograms based on the method have been prepared for both radiation-rate and radiation-exposure forecasting. In the course of this work it was found that the widely used "standard" decay of radiation from fallout should vary in time as $t^{-1.3}$ instead of $t^{-1.2}$ as presently used.

\section{Mathematical Physics}

\section{Mathematical Traseport Theory}

A sustained effort has been made over the last few years to provide a rigorous, coherent, reasonably complete and easily usable mathematical theory of transport processes. Unlike its specifically physical and/or purely computational counterparts, mathematical transport theory deals eacentially with a unique abstract model able to cover most situations of interest. Within this framework, the abstract transport equation is viewed as an initial boundary value problem of hyperbolic type with an intrinsic dissipation mechanism. In principle, the theory is aimed both at linear and nonlinear problems. Specific research bas been aimed towards (1) enlarging the clasees of kioetic

processes, boundary conditions, phase \&f ace and external constraints for which the basic isues of 


$$
39 \mid 40
$$

existence, uniqueness, positivity, and dissipativity can be proved; (2) making a more systematic and efficient use of the equivalence and factorization methods to combine the strengths of the integral equations and semigroup approaches; (3) studying in detail specific models (such as Vlasov, Lorentz, or Fokker-Planck) in order to gain insight on general features (c.g., spectral properties, eigenfunction expansions, integral representations of solutions, hydrodynamic behavior, etc.) not included yet in the abstract theory; (4) using dissipative boundary conditions as an alternative facor (together with, or instead of, absorption and dissipation through free streaming) to control the ex losive growth produced by nonlinearities; and (5) identifying patterns and behaviors within the train-port paradigm to enable the use of powerful methods of renormalization group theory and critical analysis.

\section{Qunntura Kinetics and Hydrodynanics}

One of the fundamental aspects of utmost importance to our understanding of micro- and macroscopic worlds is the connection between the dynamic kinetic and hydrodynamic levels of descriptions of a given system. While the derivation of classical kinetics and hydrodynamics from classical mechanies is rather well understood, the same is not true for their quantum counterparts. Investigating the relatiorship between kijetics and hydrodynamies for quantum systems has been one of the main research activities within a proposed interdivisional center for applied nonlinear sciences. So far, a closed algebra of operators appropriate for obtaining quantum hydrodynamic equations for the macroscopic variables (reduced description) has been successfully derived starting from the time-dependent Hartree Fock (TDHF) evolution equations for a system of fermions (exact description). Also, a canonical relationship between the TDHF system and a system of generalized Riccati equations has been identified; within reasonable limits, this relationship seems to hold independently of the particular realization of the potential. This opens the way to investigating a wide range of possible connections between the TDHF system and nonlinear phenomena of current interest such as chaos and bifurcations.

\section{Propagators for Noalinear Operator Equations}

Solving nonlinear operator equations is a very difficult task. Proving existence and uniqueness, classifying all the solutions or even finding some of them can be extremely difficult since the powerful general methods used in linear theory (e.g., Green-function methods, eigenfunction expansion) are not applicable to nonlinear equations. The most successful analytical methods (e.g., the inverse scattering transform, soliton solutions) are still limited to a restricted class of nonlinear operators, boundary conditions, and mostly to a single spatial dimension. Within the framework of the proposed center for applied nonlinear sciences, we have performed research aimed at finding new methods for solving nonlinear operator equations of significantly greater generality than presently available. This research has led to the development of a general and canonical method for solving nonlinear operator equations by converting them into integral equations whose kernels are propagators analogous to those found in linear problems. Since this new method is applicable to problems of any dimension and all types of boundary conditions, it appears that our new method significantly extends the inverse scattering transform method. Present research is aimed at applying this method to various problems for th: purpose of demonstrating both its generality and usefulness. 


\section{Radiation Transport Methods and Data}

\section{1}

\section{THE MORSE MONTE CARLO RADIATION TRANSPORT CODE SYSTEM*}

\author{
M. B. Enett ${ }^{t}$
}

(Abatract of ORNL-4972/R2, July 1984)

This report is an addendum to the MORSE report, ORNL-4972, originally published in 1975 and updated in 1983. This addendum contains descriptions of a set of nine sample problems which include the two previously published problems in order to make this document complete within itself. All pages have been three-hole punched so that users having loose-leaf copies of the original report can insert these pages in their binders; others can treat this as a third volume of ORNL-4972.

- Research supported by Defense Nuclear Areacy.

tComputing and Telecommunications Division.

\section{2}

\section{MORSE-CGA: \\ A MONTE CARLO RADLATION \\ TRANSPORT CODE WITH ARRAY GEOMETRY CAPABILITY*}

\section{B. Enmett ${ }^{\dagger}$}

(Abatract of ORNL-6174, April 1985)

The MORSE-CG computer code system has been revised to add a new geometry module MARS, which is the combinatorial geometry package that allows multiple arrays for specifying geometries. The new code system is known as MORSE-CGA where CGA indicates Combinatorial Geometry Array. The PICTURE code, which makes printer plots of 2-D slices through a combinatorial geometry mockup, has also been revised to handle the MARS array capability. This report documents the changes required in MORSE-CG and in PICTURE.
Readers are referred to Sect. M9 of the SCALE document for details on MARS and to Sect. M13 of the SCALE document for details on PICTURE

- Research sponsored by the Defense Nuclear Aseacy.

'Computing and Telecommunications Division.

23

\section{APPLCATIONS GUIDE TO THE MORSE MONTE CARLO CODE*}

\section{S. N. Cranex}

(Abetract of ORNL/TM-9355, Anguas 1985; teo beture presented at the Joint Revearch Center, lepra Eatabliahmeat of the Commisaion of the European Communitien in a course on Monte Carto Methods in Nuclear Reactor Analyzie, Ispre, Italy, October 8-12, 1984)

A practical guide for the implementation of the MORSE-CG Monte Carlo radiation transport computer code system is presented. The various versions of the MORSE code are compared and contrasted, and the many references dealing explicitly with the MORSE-CG code are reviewed. The treatment of angular scattering is discussed, and procedures for obtaining increased differentiality of results in terms of reaction types and nuclides from a multigroup Monte Carlo code are explained in terms of cross-section and geometry data manipulation. Examples of standard cross-section data input and output are shown. Many other features of the code system are also reviewed, including (1) the concept of primary and secondary particles, (2) fission neutron generation, (3) albedo data capability, (4) DOMINO coupling, (5) history file use for post-processing of results, (6) adjuint mode operation, (7) variance reduction, and (8) input/output. In addition, examples of the combinatorial geometry are given, and the new array of arrays geometry feature (MARS) and its three-dimensional plotting code (JUNEBUG) are presented. Realistic examples of user routines for source, estimation, path-length stretching, and cross-section data manipulation are given. A detailed explanation of the coupling between the 
random walk and estimation procedure is given in terms of both code parameters and physical analogies. The operation of the code in the adjoint mode is covered extensively. The basic concepts of adjoint theory and dimensionality are discussed and examples of adjoint source and estimator user routines are given for all common situations. Adjoint source normalization is explained, a few sample problems are given, and the concept of obtaining forward differential results from adjoint calculations is covered. Finally, the documentation of the standard MORSE-CG sample problem package is reviewed and on-going and future work is discussed.

-Reacarch sponsored by the Defene Nuclear Agency.

2.4

VARIANCE REDUCTION METHODS APPLIED TO DEEP-PENETRATION MONTE CARLO PROBLEMS*

$$
\text { S. N. Cramer J.S. Tage }{ }^{t}
$$

(Abstract of lecture presented at the Joint Research Center, Ispra Establishment of the Commisaion of the European Communities in a course on Monte Carlo Metbods in Nuclear Reactor Analyis, Ispra, Italy, October 8-12, 1984; also ORNL/TM-9643 (to be published)]

A review of standard variance reduction methods for deep-penetration Monte Carlo calculations is presented. Comparisons and contrasts are made with methods for nonpenetration, or reactor core, problems. Difficulties and limitations of the Monte Carlo method for deeppenetration calculations are discussed in terms of transport theory, statistical uncertainty and computing technology. The idea of importance and the realization of the importance function are covered in both general and mathematical terms. Each aspect of a Monte Carlo code calculation :s detailed, including the natural and biased forms of (1) the source description, (2) the transport process, (3) the collision process, and (4) the estimation process. General aspects of crosssection data use and geometry specification are also discussed. Adjoint calculations are examined in both the context of complete calculations and also approximate calculations for use as importance functions for forward calculations. Various methods of adjoint importance generation and its implementation are covered, including the simultaneous generation of both forward and adjoint flux in one calculation. A review of the current li.erature on mathematical aspects of variance reduction and statistical uncertainty is giver. Three widely used Monte Carlo codes MCNP, MORSE, and TRIPOLI - are compared and contrasted in connection with many of the specific items discussed throughout the presentation.

- Revearch sponecred by the Defenee Nuclese Afency.

tComputing and Telecommunications Division.

2.5

\section{APPLICATIONS GUIDE TO THE RSIC- DISTRIBUTED VERSION OF THE MCNP CODE (COUPLED MONTE CARLO NEUTRON-PHOTON CODE)*}

\author{
S. N. Cramer
}

(A hatract of ORNL/TM-9641, September 1985; also lecture presented at the Joint Rewearch Center, Ispra Establishment of the Commistion of the European Communitica in a course on Monte Carlo Methods in Nuclear Reactur Analysis, lspra, Italy, October 8-12, 1984)

An overview of the RSIC-distributed version of the MCNP code (a coupled Monte Carlo neutron-photon code) is presented. All general features of the code, from machine hardware requirements to theoretical details, are discussed. The current nuclide cross-sisicion and other data libraries available in the standard code package are specified, and a realistic example of the flexible geometry input is given. Standard and nonstandard source, estimator, and variancereduction procedures are outlined. Examples of correct usage and possible misuse of certain code features are presented graphically and in standard output listings. Finally, itemizea summaries of sample problems, various MCNP code documentation, and future work are given.

- Research sponsored by the Defense Nuclear Agency. 
2.6

\section{APPLICATIONS GUIDE TO THE RSIC-DISTRIBUTED VERSION OF THE KENO-V CODE*}

\author{
S. N. Craner
}

(Abstract of ORNL/TM-9642, September 1985; also lecture presented at the Joint Rescanch Center, Ispra Establishment of the Commission of the European Communities in course on Monte Carlo Methods in Nuctear Reactor Analysis, Ispra, Italy, October 8-12, 1984)

A review of the KENO-V multigroup Monte Carlo criticality code available from the Radiation Shielding Information Center (RSIC) at Oak Ridge National Laboratory (ORNL) is presentex. All aspects of the code are discussed, including the stand-alone and SCALE system modules and the applicable computer hardware systems. The cross-section requirements are outlined, and the available code package libraries and other necessary data are defined. The supergroup feature for use of the code on small computer systems is explained. The capabilities and limitations of the KENO array geometry description are illustrated. Various aspects of the random-walk and estimation procedures are itemized. Examples of the various calculated values of $k_{\text {eff }}$ and its statistical uncertainty are shown in standard output listings. Finally, a summary of sample problems and future work is given.

- Research sponsored by the Defense Nuclear Agency.

2.7

\section{MONTE CARLO TECHNIQUES FOR ANALYZING DEEP-PENETRATION PROBLEMS*}

\author{
S. N. Cramer J. Gonoordt \\ J. S. Hzadricket
}

(Abatract of a paper presented at the International Meeting on Advances in Nuclear Enginoering Computation Methods. Knoxville, TN, April 9-1I, 1985; Proc. Vol. 2, p. 812 (1985)]

A review of current methods and difficuities in Monte Carlo deep-penetration calculations is presented. Statistical uncertainty is discussed, and recent adjoint optimization of splitting, Russian roulette, and exponential transformation biasing is reviewed. Other aspects of the random walk and estimation processes are covered, including the relatively new DXANG angular biasing technique. Specific items summarized are albedo scattering, Monte Carlo coupling techniques with discrete ordinates and other methods, adjoint solutions, and multigroup Monte Carlo. The topic of code-generated biasing parameters is presented, including the creation of adjoint importance functions from forward calculations. Finally, current and future work in the area of computer learning and artificial intelligence is discussed with Monte Carlo applications.

- Research sponsored by the Defense Nuclear Afency.

'Centre d'Erudes Nucleaires de Seclay, 91191 Gif-Sur-Yvette Codex, France.

t Los Alamos National Laboratory, Los Alamo, NM

2.8

\section{A METHOD FOR COUPLING TWO-DIMENSIONAL TO THREE- DIMENSIONAL DISCRETE ORDINATES CALCULATIONS*}
J. L. Thompeon ${ }^{t}$
M. B. Emmett ${ }^{t}$
W. A. Rhoades
H. L. Dodd, Jr. ${ }^{\dagger}$

[Abstract of Trans. Am. Nucl. Soc. S0, 475 (1985)]

A method has been developed to couple twodimensional (2-D) to three-dimensional (3-D) discrete ordinates transport calculations. This method is useful for problems in which a 3-D Cartesian ( $X-Y-Z$ geometry) region of interest is very far from a point source. A 3-D solution from the source to the region of interest is prohibited by computing time and cost.

The problem is solved by first performing a 2-D discrete ordinates (DOT) calculation from the source past the region of interest. The results of the 2-D calculations are transformed into a boundary condition on the surface of the 3-D region of interest. This transformation or coupling method is developed and implemented in the DOTTOR computer code. The boundary condition is then used for a 3-D discrete ordinates (TORT) calculation in the region of interest. 
Simple test problems were used to evaluate the sauracy of the DOTTOR code. The evaluation indicates that the DOTTOR code produces accurate results with acceptable computing costs.

-Research sponsored by U.S. Nuciear Regulatory Commission, Office of Nuclear Requlatory Research, Contuinmedt Systems Research Branch.

University of Tennessee, Knoxwille, TN.

fComputing and Telecommraications Division.

2.9

\section{THE LINEAR NODAL METHOD FOR SHIILDING APPLICATIONS*}

\section{R. L. Crils ${ }^{\dagger}$ W. A. Rroades}

[Abetract of Trans. Am. Nucl. Soc 46, 657 (1984)]

New positive fixups have been developed for the Linear Nodal (LN) method; the method has been implemented on a vector computer and tested for shielding applications. The LN method shows considerable promise in two-dimensional calculations and perhaps even more promise in three-dimensional calculations for which the run time and memory required are roughly proportional to $1 / \Delta X^{3}$, where $\Delta X$ is an averaze mesh size.

- Research sponsored by the Defense Nuclear Agency.

'Computing and Telecommunications Division.

2.10

\section{THE EXTENSION OF THE LINEAR NODAL METHOD TO LARGE CONCRETE BUILDING CALCULATIONS•}

\author{
R. L. Cuilds ${ }^{t}$ W. A. Rboades
}

[Absiract of Trans. Am. Nucl. Soc. S0. 476 (1985)]

The linear nodal method has been implemented in the TORT code. A negative flux correction and a step limit for the weighted form of the linear nodal equations were developed. These extensions to the linear nodal method are absolutely essential to large concrete building studies. A mesh refinement study indicated that the linear nodal method has significantly smaller maximim flux errors than the weighted-diamond method previously available in TORT.

-Research sponsored by Defense Nuctar Aseacy.

${ }^{\dagger}$ Computing and Telecommunications Division.

2.11

\section{MPPACT OF "Fe ON NEUTRON PENETRATION IN THICX SODIUM-IRON SHISLDS*}

\section{Y. Fa D. T. Ingersal}

[Abatract of Trass. Am Nucl. Soc. 50, 470 (1985)]

The latest release of the VITAMIN-E multigroup cross-section library contains two different versions of neutron cross sections for natural iron, one based on ENDF/B-V MOD-I and the other on MOD-3. The difference between the two versions is that ENDF/B-V MOD-3 is the first ENDF/B iron cross-section set to include inelastic level excitation functions for ${ }^{57} \mathrm{Fe}$. Even though natural iron contains only $2.1 \%{ }^{57} \mathrm{Fe}$, it is shown that, in neutron penetration calculations through thick steel regions, ${ }^{57} \mathrm{Fe}$ can be 10 times more important than is indicated by its isotopic abundance.

- Research sponsored by U.S. DOE Division of Breeder Reactor Systems. 


\section{Radiation Shielding Applications}

\subsection{2}

\section{COMPARISON OF CANDIDATE SHIELDING MATERIALS FOR SPACE POWER REACTOR APPLCATIONS*}

\author{
W. W. Erade, Jr. D. E. Bartive
}

[Abatrect of Trans. Am. Nud. Soc. 46, 335 (1984)]

Tungsten and lithium hydride are the two materials most often mentioned in the design of shields for space power reactors. This comparison study was undertaken to examine the characteristics of other candidate shield materials for use in space power reactor systems utilizing high power ( $>1 \mathrm{MW})$, high coolant temperature $\left(1500-1800^{\circ} \mathrm{K}\right)$ reactor lesigns. The use of lithium hydride in such a high temperature system is complicated by the relatively low desired operating temperature range $\left(600-680^{\circ} \mathrm{K}\right)$ for a lithium hydride shield. Several material combinations were compared to a weight-optimized tungsten-lithium hydride shield for a typical space power reactor design/dose constraint combination. While none of the material combinations resulted in a shield as light as the tungstenlithium hydride shield, a few combinations such as tungsten and borated beryllium oxide appear promising for the high temperature environment.

-Research sponsored by U.S. Department of Energy.

\section{INTEGRAL NEUTRON AND GAMMA-RAY MEASUREMENTS OF THE LEAKAGE FLUX FROM A MOCXUP OF I.TTTLE BOY*}

\section{F, J. Meckentuder}

(Abstract of peper prasented at the Little Boy Colloquium, Los Alamos National Laboratory, Lo Alamos, NM, September 13 14, 1983)

This paper describes integral m*asurements of neutron and gamma-ray leakage fluxes from a critical mockup of the Hiroshima bomb Little
Boy at Loe Alamos National Laboratory with detector systems developed by Oak Ridge National Laboratory. Bonner ball detectors were used to map the neutron fluxes in the horizontal midplane at various distances from the mockup and for selected polar angles keeping the sourcedetector separation constant. Gamma-ray energy deposition measurements were made with thermoluminescent detectors at several locations on the iron shell of the source mockup. The measurements were performed as part of a larger program to provide benchmark data for testing the methods used to calculate the radiation released from the Little Boy bomb over Hiroshima.

-Research sponsored by U.S. DOE Office of Health and Environmental Research.

\subsection{4}

\section{APPLICATIONS OF THE THREE-DIMENSIONAL OAK RIDGE TRANSPORT CODE*}
W. A. Rloades
R. L. Childs ${ }^{t}$
M. B. Emmett ${ }^{t}$
S. N. Cramer

IAbatract of paper presented at the ANS Topical Conference on Reactor Physica and Shielding. Chicago, Illinois, September 17-19, 1984; Proc. Vol. I, p. 225 (1984)]

Features of the Three-Dimensional Oak Ridge Transport Code (TORT) are discussed. Accuracy requirements pertaining to the analysis of radiation penetration into concrete buildings are examined. Results displayed by an auxiliary graphics package are presented. Comparisons with Monte Carlo calculations are shown. The TORT results are relatively insensitize to mesh size or directional quadrature in these tests, but concrete density is seen to be of critical importance. Comparisons with the Monte Carlo calculations show good agreement in penetration through roof and floors as well as in streaming through windoris into open areas. Significant disagreement was found in shadow areas behind obstructions, however. Experiments now underway may resolve this problem area. TORT is 
based on DOT methods, and experienced DOT users find it quite user friendly.

- Research sponsored by Defene Nuclear Afency.

tComputing and Telocommunications Divisions.

2.15

\section{ANALYSIS OF THE TORT VALIDATION EXPERIMENT *}

\author{
W. A. Rroades \\ R. L. Cails $t$ \\ D. T. Ingersoll F. J. Meckentinaler
}

[Abatract of Trans. An. Nucl. Soc. 59, 473 (1985)]

Past applications of discrete ordinates calculations have dealt principally with compact systems (e.g., reactor cores or shields). As computers have become powerful enough for threedimensional discrete ordinates, however, the calculation of large interior spaces has become practical. ORNL is presently examining the radiation doses inside buildings exposed to weapon radiation during World War II. In order to test the computational methods and data, a model building has been constructed and irradiated at the ORNL Tower Shielding Facility, allowing direct measurement of fluxes within the structure. Data comparisons show good comparisons in areas more or less fed by the source, but insufficient fast neutron and gamma Iluxes behind obstacles.

- Research sponsored by Defense Nuclear Agency.

'Computing and Telocommunications Division.

\subsection{6}

\section{ANALYSIS OF NEUTRON STREAMING IN THE HTGR LOWER SUPPORT REGIONS*}

\section{O. Shater S. N. Cramer}

[Abatract of paper presented at the ANS Topical Conference on Reactor Physics and Shiclding. Chicapo, Illinois, September 17-19, 1984; Proc. Vol. II, p. 724 (1984)]

The lower core support structure of the High-Temperature Gas-Cooled Reactor (HTGR) contains many large coolant holes. The coolant holes, filled only with helium gas, allow much neutron streaming to take place. The neutron streaming contributes to higher neutron flux levels in the lower plenum. Major concern was focused on the impact of the neutzon streaming on the thermal neutron fluence at the sidewall thermal barrier cover plate. The cover plate, made of Hastelloy- $X$, has been limited to a thermal neutron fluence of $10^{17} \mathrm{~cm}^{-2}$.

Calculations were made with the DOT-IV two-dimensional discrete ordinates code and the MORSE Monte Carlo code to determine the extent of neutron streaming through the lower support regions. With the streaming paths considered, the leakage from the support blocks was higher than that for a homogeneous model by a factor greater than 1000 over most of the energy range. A factor of 2.5 streaming effect was found for thermal neutron streaming through the HTGR support post array.

The calculations of neutron streaming through the lower reflectors and support blocks were verified using results from a prototypic mockup experiment. Verification of the calculation of neutron streaming through the support post array in the lower plenum could not be achieved economically. However, an analysis of the calculational methods indicated that the calculated thermal neutron fluence at the sidewall is probably at most a factor of 2.3 conservative due to a overestimation of the thermal neutron flux leaving the support block and directed toward the sidewall. It is recommended that the corservative value be used for shield design purposes.

- Research sponsored by U.S. DOE Reactor Research and Technology Division.

2.17

\section{ANALYSIS OF PHASE I OF THE HTGR BOTTOM REFLECTOR AND CORE SUPPORT BLOCK NEUTRON- STREAMING EXPERIMENT*}

\section{O. Slater}

(Abetract of ORNL/TM-9252 (July 1984); aleo Trans. Am. NW! SOC. 46, 637 (1984)]

The results of the analysis of Phase I of an experiment to study neutron streaming through a 
mockup of the lower core support regions of GA Technologies' large high-temperature gas-cooled reactor (HTGR) were presented. The mockup was divided into seven sections plus a spectrum modifier. Phase I studied neutron transport through the spectrum modifier and the first four sections of the configuration.

The purpose of the experiment was to verify calculational methods used in the HTGR design calculations. Results from design calculations had indicated very large streaming effects and that a major design criterion, the thermal neutron fluence at the lower sidewall thermal barrier cover plate, would be exceeded by nearly a factor of 10. The calculations were performed mainly with the DOT-IV computer code and results were corrected for streaming where applicable with results from the MORSE computer code.

The above codes were used, with modified streaming correction techniques, in the analysis of the experiment. Differential fluxes were computed with DOT-IV and were streaming corrected as appropriate with results from MORSE calculations. The fluxes were folded with ietector response functions to give detector count rates which ccild be compared with measured results.

In general, the calculated detector count rates for the Phase I configurations were found to be in good agreement with the measured results. Calculation-to-experiment ratios of 0.8 to 1.2 were common. The good agreement between the calculated and measured results for the Phase I configurations provided partial confirmation of the accuracy of the methods used in the design and experiment analysis and of the results obtained using the methods.

-Research sponsored by U.S. DOE Ofince of Coaverter Reactor Deployment.
2.18

\section{A THEORY OF THE FISSION- SOURCE-DRIVEN NEUTRON NOISE FIELD*}

\section{F. C. Diflippo}

[Abstract of Trans. Ame. Nucl. Soc. 46, 738 (1984)]

The extensive use of the ${ }^{252} \mathrm{Cf}$-driven noise technique makes it desirable to develop a general theory of the fission-source-driven noise fisld. If we obtain the probability generating function (PGF) in the presence of a fission source, the equations relevant to all the noise techniques using such a source can be obtained.

The differential equation for the PGF, $F(x, t)$, of a point reactor model in the presence of a fission source can be obtained from the forward Kolgomorov equation; it is

$$
\begin{gathered}
\frac{\partial F}{\partial f}=S\left[f_{s}(x)-1\right] F+\left\{\Lambda_{c}(1-x)\right. \\
\left.+\Lambda_{f}\left[f_{F}(x)-x\right]\right\} \frac{\partial F}{\partial x},
\end{gathered}
$$

where $x$ is the dummy variable associated with the number of neutrons in the system, $S$ is the number of fissions per unit time in the source, $\Lambda_{\varepsilon}$ and $\Lambda_{f}$ are the probabilities per unit time for the capture and fission processes, respectively, and $f_{J}(x)$ and $f_{F}(x)$ are the PGF of the number of neutrons produced per fission in the source and the fissile element, respectively.

Expanding $f_{s}(x)$ and $f_{F}(x)$ in powers of $(1-$ $x)$ and keeping terms up to the second order, the asymptotic solution for Eq. (1) is

$\ln F(x, \infty)=-S_{n} /(\alpha Y J)$

$$
\ln [1+Y J(1-x)]
$$

where $S_{n}$ is the average number of neutrons per unit time produced by the source, $\alpha$ is the prompt decay constant and $Y_{1}$ is the usual correlation term.

In this way we obtain the same distribution as with the photoneutron source $(f=1)$; namely, the Polya or Negative Binomial Distribution. 
The only difference from the case with photoneutron source is the appearasce of a factor $f>1$ multiplying the correlation term $Y_{1}$. This factor is the consequence of the extra degree of randomness; now not only the disintegrations in the source are at random but also the number of neutrons per generating event. Consequently, the system is noisier than usual. The factor $f$ can be written as a function of more useful parameters in the following way:

$$
f=1+\frac{\bar{\nu}_{s}}{\bar{\nu} D} D_{s} \beta_{c} f(1+|s|),
$$

where $D_{s}$ and $D$ are, respectively, the Diven factors for the source and the fissile element, $\beta_{\text {ef }}$ is the effective delayed fraction, and $\mathbf{S}$ is the reactivity in dollar units. For a ${ }^{235} \mathrm{U}$ reactor, $f$ changes $-1 \% / \$$.

Equations (2) and (3) suggest a new method to mezsure $\beta_{e f}$; because the product $f Y_{1}$ appears instead of $Y_{1}$, we have the general result that the ratio of the measurement of the correlations in the same system, first with a fission source and then with a photoneutron source, is equal to $f$. In this way it would be possible to measure $f$ as a function of the reactivity. Then a linear fit would allow an independent measurement of $\beta_{\text {ef }}$.

Another consequence of this factorization is that all the formulas used with the different noise techniques in the case of a p'rotciscutron source can also be used to interpret experiments performed with the ${ }^{252} \mathrm{Cf}$ source. It is only necessary to substitute in these formulas an equivalent factor for the Diven factor: $D_{e q}=f D$.

- Research sponsorad by U.S. DOE Office of Breeder Reactor Systems.

\subsection{9}

\section{FLUX SYNTHESIS FOR THE ON-LINE SURVEILLANCE OF NUCLEAR POWER PLANTS*}

\section{F. C. Difilippo $\quad$ D. G. Cacuci}

\section{B. A. Worley}

[Abatract of Trans. Am. Nucl. Soc. 47, 441 (1984)]

On-line monitoring of reactivity and power distribution in reactors requires on-line determi- nation of flux, isotopic compositions, and reactivity changes as functions of burnup and operating conditions. Thus, the development of such a monitoring procedure depends on the availability of a calculational method that can efficiently incorporate reactor operating data and results from detailed off-line computations. Calculational methods based on analytical solutions to judiciously simplified neutron balance equations can offer a practically useful compromise between the computationally fast but unreliable parameterizations that describe the reactor state as a function of operating data and the more accurate but time-consuming multidimensional deterministic calculations.

This paper describes the development of a calculational method suitable for on-line reactivity and power distribution monitoring (RPDM). Underlying the development of this calculational method is the expansion of the neutron flux in a complete set of eigenfunctions corresponding to the buckling eigenvalues of a Helmholtz equation. Expansions in such eigenfunctions have previously been used for solving reactor kinetics problems, and for analyzing reactivity and flux effects induced by random dispersal of materials in a nuclear reactor. Note the important fact that the set of eigenfunctions of the Helmholtz equation is complete (and thus constitutes a basis), whereas the set of eigenfunctions associated with the $k$-eigenvalues is not complete.

The accuracy of the expansion was assessed by comparison to the exact flux and reactivities for an idealized one-dimensional cylindrical representation of the Fast Flux Test Facility (FFTF). The representation includes six regions composed of five different mixtures: inner and outer core, sodium gap, $\mathrm{B}_{4} \mathrm{C}$ control rods, and reflector. For this geometry, the eigenfunctions are the Bessel functions $J_{0}\left(\alpha_{n} r / R\right)$, where $\alpha_{n}$ are the zeros of $J_{0}(x)$ and $R$ is the external radius of the reactor.

The main conclusion that can be drawn from the comparison is that even very large reactivity changes can be efficiently estimated using perturbation theory in conjunction with only a few modes in the eigenvalue expansion. This efficient way of estimating reactivity changes is particularly useful for incorporating burnup effects as needed for the development of on-line RPDM. 
In summary, the eigenfunction expansion was found to permit efficient, fast calculation of both the flux distribution and reactivity changes due to operational decisions. These attributes make this calculational method well suited for on-line monitoring of reactivity and power distribution. Although illustrated here within the framework of one-group diffusion theory, extending the method tc multigroup formalism is straightforward. Furthermore, both experimental data and correlations from more detailed calculations can be incorporated readily in this calculational method. An important by-product of the expansion is the analytical calculation of higher static modes.

- Research sponsored by U.S. DOIE Office of Reactor Research and Technology.

2.20

\section{NEUTRONIC CALCULATIONS FOR A NEW HIGH FLUX REACTOR*}

\author{
F. C. Difilippo $\quad$ B. A. Worky \\ D. R Voady
}

(Abstract of paper presented at the International Conference on Nuclear Data for Basic and Applied Sciences, Santa Fe, NM, May 13-17, 1985)

ORNL has begun the design of a new high nux reactor to be used for basic esearch, isotope production, and material irradiation. One of the principal goals of the design is the production of a thermal flux peak in the reflector larger than $5 \times 10^{15} / \mathrm{cm}^{2} \cdot \mathrm{sec}$. None of the existing steady sources of neutrons in the world produce such neutron flux. Many calculations were performed to determine the best configuration for this reactor. Calculations show that a highly enriched ${ }^{235} \mathrm{U}$ reactor with $\mathrm{D}_{2} \mathrm{O}$ as moderator and reflector at a power level of $200 \mathrm{MW}$ would produce the desired peak flux, and the reactor would have a reasonable cycle time. The numerical model, which uses the BOLD VENTURE code, was checked by calculating the existing HFIR reactor (power, $100 \mathrm{MW}$; flux, $1-2 \times 10^{15} / \mathrm{cm}^{2} \cdot \mathrm{sec}$ ).
Concurrently, the problem of the slowingdown and diffusion of neutrons from a spherical fast source immersed in a moderator was solved analytically. This solution interrelates total power, power density, maximum thermal flux, and transport properties of the reflector. It is also possible to calculate the contributions of the neutrons moderated in the core and in the reflector relative to the total fux in the reflector.

-Rescarch sponsored by the U.S. DOE Division of Materials Sciences.

\subsection{1}

\section{STOCHASTIC PROCESSES IN A SUBCRITICAL NUCLEAR REACTOR IN THE PRESENCE OF A FISSION SOURCE*}

\author{
F. C. Diflippo
}

[Abstract of Nucl. Sci. Eng. 99, 13 (1985)]

The forward Kolgomorov equation is written for the case of a subcritical reactor monitored by two detectors and excited by a fission source located inside a fission chamber (an arrangement currently in use to measure reactivities). The marginal distribution of neutrons is shown to be given by the negative binomial distribution with an amplified correlation as compared to the case of a photoneutron source. The amplification allows the definition of an equivalent factor $D_{\mathrm{eq}}$ for the Diven factor, which makes possible the application of formulas originally derived for interpr "tation of noise measurement in the presence of a photoneutron source to the case of a fission source. The ratio of the correlations measured under the successive presence of both kinds of sources all Jws the direct measurement of the effective delayed fraction, $\beta_{\mathrm{efr}}$. $D_{\mathrm{eq}}$ is proven to be consistent with a derivation based on the Schottky prescription for the noise source.

- Research sponeored by U.S. DOE Office of Nuclear Rezulatory Revearch, Division of Facility Operationa. 


\section{THERMAL REACTOR DESIGN FOR A NEUTRON-SCATTERING SOURCE FACILITY*}

\author{
B. A. Wortey F. C. Difilippo \\ D. R. Voady
}

[Abstract of 7rans. Am. Nu-l. Soc. 49, 422 (1985)]

Material scientists have expressed interest in a neutron-scattering facility with a thermal neutron source strength corresponding to a flux of $5.0 \times$ $10^{15} \mathrm{n} / \mathrm{cm}^{2} \cdot \mathrm{s}$. The source would need to be steady over extended time periods and be accessible to experimental stations.

In an attempt to obtain a reactor design with a higher flux in the reflector, scoping calculations were performed for various core configurations, coolants, and reflector materials. The conclusions drawn from the scoping analysis are:

1. Substituting $\mathrm{D}_{2} \mathrm{O}$ for $\mathrm{H}_{2} \mathrm{O}$ as the moderator and coolant results in a higher thermal flux level in the reflector. However, use of $\mathrm{D}_{2} \mathrm{O}$ also results in an increase in the fissile loading requirements.

2. A fractional increase in the total core power increases the peak reflector flux, $\phi_{r}$, but not by the same fractional amount.

3. For the same power, reasonable changes in the core configuration increase $\phi_{r}$ by 10 to $25 \%$.

4. Decreasing the fuel-to-moderator volumetric ratio from that used in HFIR reduces the fissile loading but also decreases $\phi_{r}$.

These conclusions lead to the identification of a $\mathrm{D}_{2} \mathrm{O}$-moderated, -cooled, and -reflected design that, at a power level of $200 \mathrm{MW}(\mathrm{t})$, has a peak thermal flux in the reflector of at least $5 \times 10^{15}$ $\mathrm{n} / \mathrm{cm}^{2} \cdot \mathrm{s}$ for a 16-day full-power fueling period.

From a fuel utilization standpoint, using $\mathrm{D}_{2} \mathrm{O}$ rather than $\mathrm{H}_{2} \mathrm{O}$ as the coolant results in an undermoderated core and requires a greater inventory of fuel.
This fuel cycle cost penalty and the complications of handling $\mathrm{D}_{2} \mathrm{O}$ are negative factors that will be considered in future assessments.

-Research sponsored by U.S. DOE Office of Basic Energy Sciences.

2.23

\section{MODULAR GAS-COOLED REACTOR HEAT TRANSFER MECHANISMS*}
M. G. Sarage ${ }^{t}$
B. A. Worley

[Abstract of Trans. Am. Nucl. Soc. 46, 874 (1984)]

Recent investigations of inherently safe hightemperature gas-cooled reactors have focused on small-core concepts that do not have active emergency core cooling systems. The heat transfer mechanisms in a pebble-bed core (PBC) and a solid-block core (SBC) following a depressurized loss-of-forced-circulation heatup transient were examined.

At low temperature, the conduction of heat through solid granhite removes heat more effectively than does radia:ion and conduction in a bed of pebbles. At a higher temperature, the thermal radiation process becomes dominant.

A representative modular reactor core was modeled as both an SBC and a PBC. The heat transfer mechanism was less effective in the PBC during initial states of the transient, but as transient temperature rose above $1750^{\circ} \mathrm{C}$ (for this case), thermal radiation in the PBC became a more effective heat removal mechanism than did conduction in the SBC. The net effect was that the peak temperature was only slightly higher for the PBC.

Whether the peak temperature of a transient is greater in a PBC or an SBC was found to depend on the decay heat generation rate and the elapsed time the core experiences temperatures above the point at which the radiation process dominates.

\footnotetext{
- Research sponsored by U.S. DOE Office of Converter Reactor Deployment.

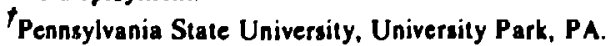




\section{A ONE-DIMENSIONAL MODELING \\ OF PADIAL HEAT REMOVAL DURING \\ DEPRESSURIZED HEATUP TRANSIENTS \\ IN MODULAR PEBBLE-BED AND PRISMATIC HIGH-TEMPERATURE GAS COOLED REACTORS*}

\author{
M. G. Saraget \\ (Abstract of ORNL/TM-9215, July 1984)
}

A one-dimensional computational model was developed to evaluate the heat removal capabilities of both prismatic-core and pebble-bed modular HTGRs during depressurized heatup transients. A correlati. $\mathrm{n}$ was incorporated to calculate the temperature- and neutron-fluencedependent thermal conductivity of graphite. The modified Zehner-Schlünder model was used to determine the effective thermal conductivity of a pebble bed, accounting for both conduction and radiation.

Studies were performed for prismatic-core and pebble-bed modular HTGRs, and the results were compared to analyses performed by GA and GE, respectively. For the particular modular reactor designs studied, the prismatic HTGR peak temperature was $2152.2^{\circ} \mathrm{C}$ at 38 hours fellowing the transient initiation, and the pebble-bed peak temperature was $1647.8^{\circ} \mathrm{C}$ at 26 hours. These results compared favorably with those of $\mathrm{GA}$ and GE, with only slight differences caused by neglecting axial heat transfer in a onedimensional radial model.

This study found that the magnitude of the initial power density had a greater effect on the temperature excursion than did the initial temperature. Neglecting the neutron fluence in the prismatic HTGR caused a $10 \%$ decrease in the peak temperature, and modeling gas gaps between prismatic fuel assemblies produced a $5 \%$ increase in the peak temperature. Replacing the prismatic core with a pebble bed resulted in a $1 \%$ increase in peak temperature.

\footnotetext{
- Recearch sponeored by U.S. DOE Office of Converter Reactor Deployment.

'Pennsylvania State Univenity, Univenity Park, PA.
}

\section{APPLICATION OF VARIATIONAL HEURISTIC LEARNING METHODS TO REACTOR PHYSICS: A NEW CONCEPT IN ARTIFICIA INTELILIGNCE*}

\author{
T. L Kiegt R. R. Perez \\ D. G. Cacenci K. T. Woodt \\ E. L. Machado'
}

[Abstract of Trens. Am. Nuch Soc 47, 439 (1984)]

Control, surveillance, and diagnosties algorithms for nuclear power plants rely on the determination of the distribution of process variables and plant parameters by the measurement of response functions such as reaction rates and control rod worths. For the past few years, a substantial effort has been made toward the solution of the above class of problems by heuristic learning methods. These methods, which utilize a minimal amount of mathematical manipulations and which are endowed with learning capabilities, are very convenient for on-line computer operation.

This work illustrates the utilization of a novel synthesis of variational and heuristic learning methods to reactor physics, whereby the machinery of variational techniques provides the heuristics for the description of the physical system.

-Research sponsord by U.S. DOE Ofice of Reactor
Rewearch and Techoology.
tUniversity of Tenneswee, Knoxville, TN.

2.26

\section{DETERMINISTIC SENSITIVTTV ANALYSIS OF TWO-PHASE FLOW SYSTEMS: FORWARD AND ADJOINT METHODS*}
D. G. Cacuel
E. Wacholdert
S. Kaizermant
N. Tomertant

(Abatract of EPRI-NP-3635, July 1984)

This report presents (a) a self-contained mathematical formalism for deterministic sensitivity analysis of two-phase flow systems, (b) a 
detailed application - which illustrates the physical meaning of this formalism - to sensitivity analysis of the homogeneous equilibrium model of two-phase flow, and (c) a representative application of this formalism to sensitivity analysis of a model (simulating "pump-trip"-type accidents in BWRs) where a transition between single phase and two phase occurs. This mathematical formalism incorporates both the forward (i.e., a generalization of the "direct") methud and the adjoint method of sensitivity analysis. In practice, for about the same amount of computation, the forward method gives the sensitivities of all variables to single parameter while the adjoint method gives the sensitivities of a functional of the variable to all parameters.

The rigor and generality of this sensitivity analysis formalism stem from the use of Gateaux (G-) differentials. In particular, the use of Gdifferentials gives precise mathematical and physical meaning to the sensitivily of a response to parameters that are func;ions rather than scalars and allows treatment of discontinuous responses, dependent variables, and r.:odel parameters.

This report highlig'its the major aspects of deterministic (forward and adjoint) sensitivity analysis, including Jerivation of the forward sensitivity equations, derivation of sensitivity expressions in terms of adjoint functions, explicit construction of the adjoint system satisfied by these adjoint functions, determination of the characteristics of this adjoint system, and demonstration that these characteristics are the same as those of the original quasilinear twophase flow equations. I nis proves that whenever the original two-phase flo'w problem is solvable, the adjoint system is also solvable and, in principle, the same numerical methods can be used to solve both the original and adjoint equations.

The uses of sensitivities are discussed and illustrated analytically and numerically. In this context, it is shown that the sensitivities accurately predict the effects of small variations in the model parameters. This accuracy is not degraded by the discontinuities that exist across the timedependent boundary between the single-phase and two-phase regions. Also, relative sensitivities are used to rank the inportance of all model parameters and to assess uncertainties in model result.

Overall, this research provides a complete theoretical framework for implementing an efficient first-order sensitivity analysis capability into codes that model two-phase flow. In particular, this research provides a proof-of-principle that scnsitivity analysis of systems undergoing a phase change can be performed using adjoint functions. This gives confidence that the traditional efficiency of adjoint methods can be advantageously used to perform sensitivity analysis of the complex two-phase flow models encountered in practice.

-Research sponsored by the Electric Power Research Institute.

'The Tectivion-Israel Institute of Tecbnology, Haifa, Israel.

\subsection{7}

\section{AN EXACT SENSITTVITY ANALYSIS OF A SIMPLIFIED TRANSIENT TWO-PHASE FLOW PROBLEM*}
E. Wacholder ${ }^{t}$
S. Kaizerman ${ }^{\dagger}$
N. Tomerian ${ }^{\dagger}$
D. G. Cacuci

[Abstract of Nucl. Sci. Eng. 89. 1 (1985)]

Two metheds of sensitivity theory, i.e., the Direct Sensitivity Approach and the Adjoint Sensitivity Method, have been successfully applied to a simplified problem of transient, onedimensional, composite region of single-phase and homogeneous equilibrium two-phase flow within a uniformly heated channel subjected to an exponential inlet now decay. I, both methods, exact analytical solutions for all elementary sensitivity coefficients at each point in space and time are obtained. A general procedure for the construction of the sensitivity equations' boundary conditions at the moving boundary between the two phases has been developed and applied. Discontinuities in the velocity and quality sensitivity coefficients across the moving boundary have been obtained. The enthalpy sensitivity coefficients are found to be continuous. The behavior of the sensitivity coefficien's has been investigated. This investigation provides insights 
into the relative importance of the input parameters and the nature of the propagation of uncertainties in space and time in two-phase flow systems.

-Research sponsored by Electric Power Research Institute tThe Technion-Israed Institute of Technology, Haife, Isreel.

\subsection{8}

\section{ADJOINT SENSITIVITY ANALYSIS OF A THERMAL-HYDRAULC SYSTEM UNDERGOING PHASE CHANGE DUE TO BOILING TRANSITION*}

\section{G. Cacuci \\ E. Wacbolder $t$ \\ S. Kaizermant \\ N. Tomerian't}

[Abstract of Trans. Am. Nucl. Soc. 46,870 (1984)]

Sensitivity analysis of practical problems can be performed systematically and very efficiently by using adjoint functions. In areas of interest to nuclear reactors, this efficiency has been amply demonstrated on several widely used codes for neutronics and/or thermal-hydraulic calculations.

Applications of the adjoint method of sensitivity analysis to models involving phase transitions, where nondifferentiability occurs, do not seem to have been repnited to date. The purpose of this paper is to report results from a successful adioint sensitivity analysis of a space- and timedependeril system where phase transition occurs due to boiling. The specific model chosen for this analysis is a simplified but representative model of a boiling water reactor (BWR) "pump trip"-type accident. This $m$ it is of particular importance to BWR safety siilce pump failure is one of the most limiting hypothetical accidents in BWRs.

Using adjoint functions for this model, all sensitivities have been calculated and used to predict changes that occur in $h, u$, and $x$ when the parameters $\alpha_{i}$ are varied by $\Delta \alpha_{i}$. For perturbations of up to $10 \%$, these predicted changes agree well with the actual changes calculated by using the actual perturbed parameter values $\alpha_{i}+$ $\Delta \alpha_{i}$. The accuracy of these predictions is not degraded by discontinuities ac-oss the boiling boundary.
Overall, this work provides a proof-ofprinciple that sensitivity analysis of systems undergoing a phase change can be performed using adjoint functions. This gives confidence that the traditional efficiency of adjoint methods can be exploited for sensitivity analysis of the complex two-phase flow models encountered in practice.

-Research sponsored by Electric Power Research Institute

'The Technion-Isreel Institute of Technotogy, Haifa, Israel.

\subsection{9}

\section{NONLINEAR DYNAMICS AND STABILITY OF BOILING WATER REACTORS. I: QUALTATTVE ANALYSIS*}

\section{$\begin{array}{ll}\text { J. March-Leolat } & \text { D. G. Cacaci }\end{array}$}

\section{R. B. Perez}

\section{[Abstract of Nucl. Sci. Eng. (in press)]}

A phenomenological model has been developal to simulate the qualitative behavior of bo.ting water reactors in the nonlinear regime under deterministic and stochastic excitations. After the linear stability threshold is crossed, limit cycle oscillations appear due to interactions between two unstable equilibrium points and the phase-space trajectories. This limit cycle becomes unstable when the feedback gain exceeds a certain critical value. Subsequent limit cycle instabilities produce a cascade of period-doubling bifurcations that leads to aperiodic pulsed behavior.

Under stochastic excitations, boiling water reactors exhibit a single characteristic resonance, at approximately $0.5 \mathrm{~Hz}$, in the linear regime. By contrast, this work shows that harmonics of this characteristic frequency appear in the nonlinear regime. Furthermore, this work also demonstrates that amplitudes of the limit cycle oscillations do not depend on the variance of the stochastic excitation, and remain bounded at all times.

- Rescarch aponeored by U.S. Department of Energy.

tUniversity of Tennescee, Knoxville. TN. 
2.30

\section{NONLINEAR DYNAMICS AND STABIITY OF BOILING WATER REACTORS. II: QUANTITATIVE ANALYSIS*}

\section{J. March-Leaba' ${ }^{\prime}$ R. B. Perez}

D. G. Cacuci

[Abstract of Nucl. Sci. Eng. (in preas)]

A physical model of nonlinear boiling water reactor dynamics has been developed and employed to calculate the amplitude of limit cycle oscillations and their effects on fuel integrity over a wide range of operating conditions in the Vermont Yankee reactor. These calculations have confirmed that, beyond the threshold for linear stability, the reactor's state variables undergo limit cycle oscillations. This work shows that the amplitude of these oscillations are very sensitive to changes in operating condition and are not restricted to small magnitudes as observed in previous stability tests. Consequently, large-amplitude limir cycle oscillations become a possible scenario for boiling water reactor operation at low-flow conditions. Tne effects on fuel integrity of such large-amplitude oscillations have been studied in detail. In particular, it has been shown that limit cycles that oscillate with frequencies higher than $0.25 \mathrm{~Hz}$ and that reach the high-power safety scram level of $120 \%$ are not likely to compromise fuel integrity.

- Revearch sponsored by U.S. Department of Energy.

tUniversity of Tennessec, Knoxvilie, TN.

\section{CALCULATION OF LIMIT CYCIE AMPLTUDES IN COMMERCIAL BOILING WATER REACTORS*}

\section{J. Marcb-Leaba' $\quad$ R. B. Perez}

\section{G. Cacued}

[Abetract of Trans. Am. Nucl. Soc. 46, 749 (1984)]

This paper describes an investigation of the dynamic behavior of a BWR in the nonlinear region corresponding to linearly unstable condi- tions. For this purpose, a nonlinear model of a typical BWR was developed. The equations underlying this model represent: (a) a onedimensional void reactivity feedback (described by a 12-node axial expansion of the energy, continuity, and momentum equations for the average channel), (b) point kinetics with a single delayed-neutron group, (c) fuel behavior (obtained by using a two-node representation of pellet and cladding behavior), and (d) recirculation loop dynamics (described by a single-node integral momentum equation). Note that the nonlinear terms appear in the point kinetics and momentum equations.

Important conclusions of the research described in this work are: (a) the nonlinear model developed successfully predicts the appearance of the experimentally observed limit cycles, (b) the amplitude of the power oscillations in the nonlinear region is very sensitive to changes in the operating conditions, and (c) limit cycles of large amplitude, but not so large as to cause an automatic scram, are a possible scenario for BWR plant operation.

-Research sponsored by U.S. DOE Office of Reactor Research and Technology.

'University of Tennessec, Knoxville, TN.

2.32

\section{A STOCHASTIC MODEL TO MONTTOR MECHANICAL VIBRATIONS IN PRESSURIZED WATER REACTORS*}
R. T. Woodt
J. March-Leobat
R. B. Perez
F. J. Sreeseyt

[Abstract of Trans. Am. Nucl. Soc. 46, 735 (1984)]

An algorithm based on a model of the interaction between small mechanical motions and reactor neutronics was developed and used to investigate the structure of neutron PSDs from Sequoyah-1. The algorithm adequately describes the signature in terms of identifiable parameters and it separates the resonant components of the PSD. These capabilities proved useful in quantifying the variation in vibrating frequencies over the fuel cycle and in identifying the core barrel resonance even when it was masked by the 
increased pole strength of the second fuel mode. Based on these results, this algorithm may offer a method to quantitatively monitor and interpret the evolution of a PWR's noise signature.

- Recarch spomorod by US. DOE Offioe of Reactor Revearch and Techoolozy.

University of Tennewee, Knoxville, TN.

Flnstrumentation and Controts Division.

\section{BENCHMARK PROBLEM SOLUTION: TWO-DIMENSIONAL PWR BURNUP PROBLEM WTTH FUEL MANAGEMENT TREATTNG TWO CYCLES*}

\author{
D. R. Voady
}

\begin{abstract}
[Abstract of a Benchm + Problem in Argonse Code Center Benchmark Problem Dook, ANL-7416 (1985); aleo paper entitled Solution of a Two-Dimensional PWR Benchmark Problem Modeling Two Cycles with Refueling and Fuel Assembly Repositioning by Finite-Difference Method," presented at the International Meeting on Advances in Nuclear Enginecring Computation Methods, Knoxville, TN, April 9-1I, 1985, Proc. Vol. I, p. 159 (1985)]
\end{abstract}

An interesting reactor core history problem was submitted from KWU in the FRG to the ANS Mathematics and Computation Division benchmark problem eftort. Results were obtained to this problem applying local finitedifference diffusion theory and exposure capability [D. R. Vondy et al., ORNL-5711 (June 1981)]. The key modeling variables of numbers of (a) meshpoints, (b) exposure zones, and (c) timesteps were varied. Also of some interest was the attempt to improve the accuracy with a redepletion procedure. Benchmark quality results were generated for publication in the formalized program. These may be compared with results obtained at KWU applying advanced and sophisticated nodal neutronics methods. The problem is somewhat simplified in that cross sections were fixed arviding the impact from plutonium buildup that would usually be treated. Still rather interesting information comes from the different methods of modeling. In particular, upon refueling, the fuel elements are repositioned and several are rotated, causing the second cycle to be sensitive to the accuracy of the variation in the nuclide concentrations across the fuel assemblies. The ability of local diffusion theory neutronics capability to establish the required critical soluble boron concentration in the coolant with a direct outer-iteration procedure (instead of solving for $k$ ) is demonstrated.

-Research sponsored by U.S. Department of Energy.

\section{4}

\section{THR-TH: A HGH-TEMPSRATURE GAS-COOLED NUCLEAR REACTOR CORE THERMAL HYDRAULICS CODE*}

\section{R. Voady}

(Abstract of ORNL-5951, July 1984)

The ORNL version of PEBBLE, the (RZ) pebble bed thermal hydraulics code, has been extended for application to a prismatic gas cooled reactor core. The supplemental treatment is of one-dimensional coolant flow in up to a threedimensional core description. Power density data from a neutronics and exposure calculation are used as the basic information for the thermal hydraulics calculation of heat removal. Twodimensional neutronics results may be expanded for a three-dimensional hydraulics calculation. The geometric description for the hydraulics problem is the same as used by the neutronics code. A two-dimensional thermal cell model is used to predict temperatures in the fuel channel. The capability is available in the local BOLD VENTURE computation system for reactor core analysis with capability to account for the effect of temperature feedbask by nuclear cross section correlation. Some enhancements have also been added to the original code to add pebble bed modeling flexibility and to generate useful auxiliary results. For example, an estimate is made of the distribution of fuel temperatures based on average and extreme conditions regularly calculated at a number of locations.

- Research sponsored by U.S. DOE Office of Nuclear Power Systems. 
2.35

\section{AQ-MW(b) HIGH-TEMPERATURE \\ REACTOR CORE POWER DENSITY STUDY*}

\section{R. Voady}

(Abstract of ORNL/TM-9099, Sepeember 1984)

This study wes done to estimate the effects of reducing the design power density of a 2240-MW(th) high-temperature gas-cooled reactor. Core history and thermal hydraulics calculations were performed for average power densities of 5.8 and $7.2 \mathrm{~W} / \mathrm{cm}^{3}$, and the use of highly enriched fuel was considered. The fuel temperature conditions for the higher power density were found to be only moderately elevated at normal operating conditions. Economic considerations associated with changes in coice performance, core size, and coolant pumping requirements were assessed.

-Research sponsored by U.S. DOE Office of Converter Reactor Deployment.

2.36

\section{A MULTIGRID ITERATION SOLUTION PROCEDURE FOR SOLVING TWO-DIMENSIONAL SETS OF COUPLED EQUATIONS*}

$$
\text { D. R. Voady }
$$

(Abstract of ORNL/TM-9030. July 1984)

A procedure of iterative solution was coded in Fortran to apply the multigrid scheme of iteration to a set of coupled equations for solving twodimensional sets of coupled equations. The incentive for this effort was to make available an implemented procedure that may be readily used as an alternative to overrelaxation, of special interest in applications where the latter is ineffective. The multigrid process was found to be effective, although not always competitive with simple overrelaxation. Implementing an effective and flexible procedure is a time-consuming task. Absolute error level evaluation was found to be essential to support methods assessment. A code source listing is presented to allow simple application when the computer memory size is adequate, avoiding data transfer from auxiliary storage. Included are the capabilities for one-dimensional rebalance and a driver program illustrating use requirements. Feedback of additional experience from application is anticipated.

- Recearch sponecred by U.S. DOE OIToc of Converter Reactor Deployment.

\subsection{7}

\section{A MULTIGRID ITERATION SOLUTION PROCEDURE FOR SOLVING THREE- DIMENSIONAL SETS OF COUPLED EQUATTONS*}

\section{R. Voady}

(Abetract of ORNL/TM-9248, August 1984)

A procedure of iterative solution was coded in Fortran to apply the multigrid scheme of iteration to a set of coupled equations for threedimensional problems. The incentive for this effort was to make available an implemented procedure that may be readily used as an alternative to overrelaxation, of special int serest in applications where the latter is ineffective. The multigrid process was found to be effective, although noncompetitive with simple overrelaxation for simple, small problems. Absolute error level evaluation was used to support methods assessment. A code source listing is presented to allow ready application when the computer memory size is adequate, avoiding data transfer from auxiliary storage. Included are the capabilities for one-dimensional rebalance and a driver program illustrating use requirements. Feedback of additional experience from application is anticipated.

- Research sponsored by U.S. DOE Office of Converter Reac tor Deployment. 


\section{REGARDING OVERRELAXATION FOR ACCELERATTNG AN ITERATION PROCESS*}

\section{R. Voady}

(Abatact of ORNL/TM-8922, Jane 1984)

The solution for a vector that satisfies a set of coupled equations is often obtained economically by iteration. Application of an overrelaxation coefficient to augment the calculated iterate changes is done to accelerate the rate of convergence. This scheme is simple to implement and often effective. Much is known theoretically about the iterative behavior when the system of equations is linear, although there are complexjties that are not widely known. Extensive use is made of the scheme even to non-linear systems of equations where behavior depends on the situation. Of much concern to the developer of solution methods (typically an engineer or applied mathematician) is implementing an effective procedure at a modest investment in development and testing. Applications are described to thermal cell and neutron diffusion modeling.

- Research sponsored by U.S. DOE Office of Converter Reactor Deployment.

2.39

\section{THE DEPTH-CHARGE STATIC AND TIME-DEPENDENT PERTURBATION/SENSITIVITY SY'STEM FOR NUCLEAR REACTOR CORE ANALYSIS*}

\section{J. R. White ${ }^{t}$}

(Abstract of ORNL/CSD.78/RI, April 1985)

This report provides the background theory, user input, and sample problems required for the efficient application of the DEPTH-CHARGE system a code block for both static and timedependent perturbation theory and data sensitivity analyses. The DEPTH-CHARGE system is of modular construction and has been implemented within the VENTURE-BURNER computational system at Oak Ridge National Laboratory. The DEPTH module (coupled with VENTURE) solves for the three adjoint functions of Depletion Perturbation Theory and calculates the desired time-dependent derivatives of the response with respect to the nuclide concentrations and nuclear data utilized in the reference model. The CHARGE code is a collection of utility routines for general data manipulation and input preparation and considerably extends the usefulness of the sysiem through the automatic generation of adjoint sources, estimated perturbed responses, and relative data sensitivity coefficients. Combined, the DEPTH-CHARGE system provides, for the first time, a complete generalized first-order perturbation/sensitivity theory capability for both static and time-dependent analyses of realistic multidimensional reactor models.

This current documentation incorporates minor revisions to the original DEPTHCHARGE documentation (ORNL/CSD-78) to reflect some new capabilities within the individual codes. Most of the modifications, however, are transparent to the user. Changes that are under user control are described in the updated General Input Description and deal mainly with the processing and manipulation of data sensitivity files. Finally, it should also be noted that the sample problem input files have been modified to reflect these changes and to include the job control instructions for execution from a complete BOLD VENTURE-IV program package.

- Research sponsored by U.S. Department of Energy.

'University of Luwell, Lowell, MA.

\subsection{0}

\section{DEVELOPING NUCLEAR POWER FOR SPACE APPLICATIONS*}

\section{E. Bartine}

(Abatract of peper prisented at the ORNL Colloquium, Oak Ridge. TN, August 13, 1984)

Emerging requirements for high power levels over long time periods have revived interest in the 
use of nuclear reactors in space. Potential applications include large space platforms in geosynchronous orbit, propulsion for deep space probes, long-term lunar and planetary bases, space-based radars for air defense, and space-based bram weapons for ballistic missile defense. An R\&D program known as SP-100 has been formed jointly among NASA, DOE, and DARPA (the Defense Advanced Research Projects Agency) with a near-term goal of developing a $100-\mathrm{kW}$ space reactor and a longer-term goal of a multimegawatt system. Size and weight restrictions will necessitate compact, high power density systems, requiring $\mathrm{f}$ ighly advanced technology to insure safe, reliable multiyear remote operation. Potentially applicable Energy Systems' capabilities include reactor systems design and development, component testing, high-temperature materials, instrumentation and control systems, mechanical energy storage (flywheels), ard shielding.

- Research sponsored by ORNL Discretionary Fund.

2.41

\section{EVALUATION OF THE DIF3D NODAL OPTION FOR HTGR NEUTRONIC APPLICATIONS*}

\author{
B. R. Fran't J. R. Witet
}

[Abstrect of Trans. Am. Nucl. Sac. 4, 436 (1985)]

The hexagonal nodal method implemented in DIF3D has been shown to be faster than standard finite difference methods for he same degree of accuracy. The main conclusion from this evaluatio. is that the DIF3D nodal option is clearly the mo-e efficient tool for HTGR neutronics computations. The capability to calculate accurate power distributions at relatively low cost for large hexagonal cores should prove useful in the analysis of HTGR reactor cores.

- Research sponsored by U.S. DOE OTtice of Cunverter Reactor Deployment.

'University of Lowell, Lowell, MA.

\section{J. R. White ${ }^{t}$ T. J. Conitut}

\section{B. R. Frank ${ }^{t}$}

[Abstract of Trans. Am. Nucl. So 49, 220 (1985)]

Significant enhancements and extensions have been made to the exact analytic solution methodology. One particular highlight has been the generalization of the basic HAREM (for HARmonic Expansion Method) methodology to treat the generalized fixed-source problem that arises in generalized perturbation theory (GPT) applications.

- Research sponsored by U.S. DOE Office of Converter Reactor Ieployment.

'University of Lowell, Lowell, MA.

2.43

\section{FEW-GROUP THREE-DIMENSIONAL HEXAGONAL GEOMETRY HTGR BENCHMARK PROBLEM*}

\author{
B. R. Frank \\ J. R. White'
}

[Abstract of paper presented at the International Meeting on Advances in Nuclear Engineering Computution Methods. Knoxville, TN, April 9.11, 1985; Proc. Vol. 1, p. 194 (1985)]

The three-dimensional HTGR Benchmark Problem was solved using two different mathematical models: standard finite difference and a hexagonal nodal technique.

- Research sponsored by U.S. DOE Office of Converter Reactor Deplojment.

'University of Lowell, Lowell. MA. 
244

\section{ASSESSING APPLICATIONS OF SAUER'S METHOD FOR CALCULATING DANCOFF FACTORS*}

\section{R. D. Timerman}

(Abstract of paper presented at the 1985 Eastern Regional Student Conference, Geinesville, FL, April 5-6. 1985)

A discrepancy in calculating Dancoff factors using Sauer's geometrically dependent rational approximation has been noted for resonance calculations involving widely spaced fuel rods in graphite blocks. For a constant fuel rod radius of $1.175 \mathrm{~cm}$, Dancoff factors calculated using Sauer's approximation were found to increase with fuel rod pitch. Since the Dancoff factor is defined as the free-flight transmission probability through the moderator, an increase in moderator thickness should decrease the probability of a neutron avoiding a collision. Hence, for the range of fuel rod pitches considered, Sauer's approximation is yieiding non-physical results.
A scoping study has been carried out in which Sauer's approximation for the Dancoff factor was compared to results obtained using Wigner's non-geometrically dependent rational approximation as well as the code SUPERDAN which employs a double numerical integration technique. For this study, the results obtained for SUPERDAN were taken as the "correct" results since its methods are the most rigorous and have been shown to agree closely with Monte Carlo results. The study uncovered a range of moderator to fuel volume ratios where Sauer's method catastrophically breaks down. Further analysis yielded a relatively simpie test to determine if Sauer's method may be used for a particular application. The test was found to accurately assess applications for which Sauer's method is valid.

- Research sponsored by U.S. DOE Office of Converter Reactor Deployment.

University of Tennessee, Knoxville, TN.
2.45

\section{THE ANALYSIS AND EVALUATION OF RECENT OPERATIONAL EXPERIENCE FROM THE FORT ST. VRAIN HTGR*}

$$
\text { D. L. Moses W. D. Laming }{ }^{\dagger}
$$

(Abstract of paper presented at the Intirnational Atomic Energy Agency Specialist's Mating on Safely and Accident Analysis for Gas-Cooled Reactors, Oat Riffe. T: :, May I3 15. 1985)

The U.S. Nuclear Regulatory Commission's Oifine ivi Aulalysis and Evaluation of Operational Data has established an extensive program for screening, analyzing, and evaluating the operational experience data from all commercial nuclear power plants in the United States. This program is designed to provide feadba $2 k$ from field experience with actual operating events to the NRC's continuing efforts to assure the public's health and safety. Oak Ridge National Laboratory provides technical assistance to AEOD to evaluate the operating experience for Fort St. Vrain. The operating experience discussed includes notable safety-related events which have occurred since late 1981. Different events have been grouped according to the major plant system affected by the occurrence, including primary coolant system. electrical systems and reactor building.

- Research sponsored by U.S. Nuclear Regulatory Cummision, Orfice of Analysis and Evaluation of Operational Date.

${ }^{\dagger}$ Nuclear Regulatory Commisuion. Washington, $D C$. 


\section{Studies of Nuclear Weapons Effects}

\section{ADJOINT TRANSPORT CALCULATIONS FOR SENSITTVITY ANALYSLS OF THE HIROSHIMA AIR-OVER-GROUND ENVIRONMENT*}

\author{
$\begin{array}{ll}\text { B. L. Broadheadt } & \text { D. G. Caceaci }\end{array}$ \\ J. V. Pace, II' ${ }^{\dagger}$
}

[Abstract of Trans. Am Nucl. Soc. 46, 638 (1984)]

The U.S. Dose Reassessment Program includes reexamination of all the stages of the Hiroshima event, from the initial assembly of the fissionable material, through the transport of neutrons and gamma rays following the explosion, to the final ceposition of the dose of ionizing radiation. Such a comprehensive reassessment of the A-bumb dosimetry should lead not only to improved estimates of survivor doses, but also to defensible and reduced uncertanties in these doses. For this purpose, it is necessary to perform systematic uncertainty analyses for all links of the overall Dose Reassessment Program, and to combine - in a consistent way - the results obtained from these analyses.

This paper is the first report of results from adjoint calculations in the Hiroshima air-overground environment. The results of these adjoint calculations are currently being used to obtain the sensitivities of the neutron and gamma terma responses is $n$ ll parameters ins.' ived in the airover-ground zransport salculations. These selasitivities will then be used to rank the importano: of these parameters, and to assess the urcertaitities in the kerma responsis due in uncertaintics in these parameters. The results reported herein represent a first step toward the syiternatic sensitivity/uncertainty analysis needied to obtsin inproved estimates and reduced uricertainties for survivor doses.

- Research sponsored hy U.S. IVOE Office of Environmestal Rexearch and Development.

${ }^{\prime}$ Computiug and Telesommunications Divisins
2.47

\section{IN THE SHADOW OF GROUND ZERO*}

\author{
C. M. Hanlan
}

(Abstract of paper presentod at the Annual Meeting of the American Civil Defense Association, Daytona Beach, FL, November (5-16, 1984)

A brief historical review is presented on the development of nuclear weapons starting with the detonation of the A-bombs on Japan. A brief overview of nuclear weapon effects is given, including the nuclear winter effect. Close-in effects of EMP, initial nuclear radiation, thermal radiation, and blast are discussed in some detail. Special consideration is given to methods by which people can improve their chances for survival if they are not in shelters, even if ground zero is only a few miles away, providing the yield is a megaton or less. The concept of rings-ofhazard is introduced as a useful training method for emergency workers and decision-makers.

- Recesich sponsored jointly by the U.S. Department of Energy and the Federal Emergency Management Agency.

\subsection{8}

A GRAPHICAL METHOD FOR FORECASTTNG RADIATION EXPOSURE FROM MULTI-AGED FALLOUT FROM NUCLEAR WEAPONS*

\section{M. Henlad}

[Abatract of Health Physics (in preas)]

Afl:-r a nuclear attack it may be necessary for e...erency workers, such as firemen, utility work$\Leftrightarrow r s$, dild inedical personnel, to perform urgent iasks in areas highly contaminated by radioactive falicu:. In order tn control the radiation exposure of these worke;s it will be esseniial to provide mears to forecasi radiation exposures both inside anc outiside the fallout shelter. The method 
described in this paper is intended for use during the first few days to weeks after the attack, after which time more sophisticated methods may become available. This method requires only a survey meter such as the CDV-715, special graph paper, and a timepiece. Communications with Emergency Operating Centers or other sources of information are not necessary. The method permits the determination of the unit-time reference exposure rate and future exposure rates for a location that might be subjected to a number of different fallout clouds, without requiring knowledge of the weapon yields or times of detonation. This method will not work if differentaged fallout clouds arrive simultaneously.

-Research sponsored jointly by the U.S. Department of Energy and the Foderal Emergency Management Agency.

2.49

\section{A REVIEW OF THE NUCLEAR WINTER SCENARIO*}

\section{M. Hanland}

(Abstract of paper presented at the 1985 WATTec Conference, Knoxville, TN, February 12, 1985)

A brief history is presented of large studies on the environmental and biological consequences of nuclear war, becinning with programs sponsored by the Atomic Energy Commission in the 60s, and culminating with the Nuclear Winter scenario introduced in 1983. The rise and fall of various doomsday scenarios generated during the same period are also reviewed. Some of the key assumptions of the Nuclear Winter scenario are discussed, including smoke formation, scavenging processes, and atmospheric modeling. Possible options for superpowers to avoid Nuclear Winter that might result from a nuclear war include (1) confining the war to space (Star Wars), (2) develnping ground-penetrating warheads to destroy hardened military targets, (3) using conventional instead of nuclear explosives, and (4) avoiding all possibilities of war. Civil defense measures can still be highly effective for saving lives even if the worst Nuctear Winter scenario should become a reality.

- Research sponsored jeintly by the U.S. Departmeat of Energy and the Foderal Emergency Management Agracy.

2.50

\section{REPLY TO VICTOR N. EVDOKIMOFF*}

\section{M. Haaland}

[Abstract of Health Phys. 511 (1985)]

A response is given to a letter by Evdokimoff (Health Phys. 48, 510-511) in which he criticizes an article by Haaland (Health Phys. 46, 347-359) as being "absurd" because "it supposes that the United States can survive hundreds or thousands of nuclear weapons with an ideal civil defense program." Several studies are cited which indicate that a nationwide civil defense program could reduce U.S. fatalities in a large nuclear war from 60 to 80 percent of the population to 10 to 20 percent. A brief review of the Soviet civil defense program is given. It is estimated that the Soviet Union has been spending 30 to $\mathbf{4 0}$ times more per year on civil defense than the United States, spending possibly as much as $\$ 6$ billion in 1982.

- Reacarch sponsored jointly by the U.S. Department of Energy and the Federal Emergency Management Agency.

2.51

\section{FORECASTING RADIATION EXPOSURE FROM FALLOUT CAUSED BY MULTIPLE, NONSIMUI.TANEOUS, UPWIND GRNUND BURSTS*}

\section{M. Haaland}

[Abatract of Healih Physics 46(2), 347 (1984)]

A large-scale nuclear attask on the United States would probably result in the deposition of rartioactive failout from many ground bursts detonated at different tiri'ss. Previous methods for forecasting radiation levels and cumulative 
exposure do not provide analytical solutions for dealing with such radiation sources. A new method is presented that will allow the forccasting of radiation exposure from the fallout caused by any number of nonsimultaneous, upwind ground bursts.

- Research sponsored joindly by the U.S. Department of Erergy and the Foderal Emergency Management Afency.

2.52

\section{SHOULD WE PROTECT OURSELVES FROM NUCLEAR WEAPONS EFFECTS?:}

\section{M. Haaland}

(Abstract of paper presented at the 1984 Annual Meeting of the American Physical Society, Detroit, MI, March 27, 1984)

Simple buried-pole shelters of Soviet design have been found to withstand 100-psi overpressure in American tests. The radius $R$ in miles at which the overpressure is $P$ in psi from a groundburst of yield $Y$ in megatons is given by $R=6 Y^{1 / 3} /\left[P-0.02(P-4)^{2}\right]$ for $0.5<P<500$. In those few areas in the U.S. where conditions might support a firestorm, safe shelters could be constructed based on lessons learned from the Hamburg firestorm of 1943 in which $85 \%$ of the 280,000 people within the firestorm survived. If the people of Hiroshima had been in simple buried-pole shelters, not one person need have perished from weapon eff-cts even at ground zero where the overpressure was only 40 psi because the weapon was airburst to increase blast effects. Soviel propaganda for exterior consumption attempts to persuade our people that civil defense is not possible, whereas their internal line stresses that "there is not and can never be a weapon from which there is no defense." Despite the fact that individuals can be protected from nuclear weapon effects, the gloominess of a nationwide picture of the U.S. after a large attack indicates that a multi-layer missile defense is required in addition to civil defense.

-Research sponsured jointly by the U.S. Depertment of Energy and the Federal Emergency Management Agency.

\section{A SIMPLE, GRAPHICAL METHOD FOR FORECASTING RADIATION EXPOSURE FROM MULTI-AGED FALLOUT FROM NUCLEAR WEAPONS*}

\author{
C. M. Healand
}

(Abstract of paper presented at Health Physics Society Anaual Meeting, New Otieans, LA, June 3-8, 1984)

After a nuclear attack it may be necessary for emergency workers, such as firemen, utility workers, and medical personnel, to perform urgent tasks in areas hizhly contaminated by radioactive fallout. In order to control radiation exposure to these workers, it will be essential to provide means for the RMEW (Radiological Monitor for Emergency Workers) to forecast radiation exposures both inside and outside the fallout shelter. The method described in this paper requires only that the RMEW possess a survey meter such as the CDV-715, special graph paper, and a watch. Communications with Emergency Operating Centers or other sources of information are not necessary. The method permits the determination of the unit-time reference exposure rate and future exposure rates for the location for a number of different fallout clouds without requiring knowledge of the weapon yields or times of detonation. This method was developed in connection with the preparation of a handbook for FEMA for radiation safety for emergency workers.

- Research sponsored jointly by the U.S. Department of Energy and the Federal Emergency Management Agency.

\subsection{4}

\section{IMPROVING RADEF*}

\section{R. N. Thurmer}

(Abstract of paper presented at the Annual Mecting of the American Civil Defense Association, Daytona Beach, FL, November is 16, 1984)

A report is given of the changes being made in the Federal Emergency Management Agency's 
existing Radiological Defense (RADEF) System, including new concepts in Radiological Protection planning and the incorporation of FEMA's Integrated Emergency Management System (IEMS). In the past, RADEF has principally dealt with nuclear-attack preparedness. One of the objectives in restructuring of the program is to make the facilities, instrumentation, and staff of the present RADEF System available for use in dealing with peacetime radiological emergencies. In response to IEMS, the improved program will now be called Radiological Protection and will include preparedness and response to all radiological hazards that threaten a jurisdiction - including nuclear attack.

- Recearch sponsored jointly by the U.S. Departmeat of Energy and the Federal Emergency Managemeat Ageacy.
2.55

\section{ON THE SPECTRAL DECOMPISITION OF THE TRANSPORT OPERATOR WITH ANISOTROPIC SCATTERING AND PERIODIC BOUNDARY CONDITIONS}

\section{Protopopescu}

[Abstract of Transp. Theory Stat. Phys. 14(1), 103 (1985)]

The time-dependent linear monoenergetic transport equation with linearly anisotropic scattering is considered in slab geometry with periodic boundary conditions. Adapting a result of Friedrichs, it is shown explicitly that the spectral theorem holds for the corresponding operator, for all but a set of zero measure in the space of parameters.

\subsection{6}

\section{TIME-DEPENDENT TRANSPORT AS CRITICAL PHENOMENON*}

\section{Protopopescu W. Krein ${ }^{\dagger}$ \\ T. Keyest}

(Abstract of paper presented at the S3rd Statistical Mechanics Meeting, Rulgers University, May 9-10, 1985)

A time-dependent linear transport problem is analyzed via new integral equation renormalization group technioues and accordingly described with the language of critiral phenomena.
Relevant critical exponents and scaling relations are discussed.

-Work partially sponsored by U.S. Department of Energy.

'Department of Physics, Harvard University, Cambridge, MA.

Fepartment of Chemistry, Boston University, Boston, MA.

\subsection{7}

\section{THE GOLDSTEIN-MCKEAN MODEL REVISTTED}

\section{Protopopescu T. Keyes}

[Abstract of Physica A. 132, 421 (1985)]

The main properties of the simplest spatially nonhomogenous kinetic equation (the GoldsteinMcKean model) are briefly reviewed. The relationships with generalized and/ur extended hydrodynamics are looked upon, as they result from projection operator method, spectral analysis, and scaling. New interesting features of a broader, principal interest are highlighted in connection to the irreversible behavior of the system and to the scaling procedure. For systems with boundaries, one derives systematically the full set of hydrodynamic boundary conditions, up to any order.

- Boston University, Boston MA. 


\section{KINETIC EOUATIONS WITH REFLECTING BOUNDARY CONDITIONS*}

\section{V. M. van der Mee $\quad$ V. Protopopescu}

[Abstract of SIAM J. Math Amal. (in preas)]

A general abstract model of time-independent kinetic equations on the half-line is presented. The existence and uniqueness of the solution is proved under specified incoming flux and nonmultiplying boundary reflection processes. An iterative method is formulated for computing in principle the solution by using the solution of the analogous problem without reflection. In many concrete cases (e.g. neutron transport, BGK model in rarefied gas dynamics, etc.) the available explicit expression for the latter provides the actual solution of the general problem. Possible generalizations and open problems are briefly discussed.

- Research partially supported by U.S. Department of Energy and National Science Foundation.

tVirginia Polytechnic Institute and State University, Blacksburg, VA.

\section{HALF-RANGE SOLUTIONS OF INDEFINITE STURN-LIOUVILLE PROBLEMS}

\section{Klans" C.V.M. van der Mee ${ }^{\dagger}$ V. Protuperiescu}

[Abatract of C: R. Acad. Sc. Paris 300, 165, Series I (1985); also J. Funct. Anal. (in press)]

We consider diffusion equations of the Sturm-Liouville type and construct their solutions in two different ways: (1) by means of an eigenfunction expansion and (2) by using an integral equation and Wiener-Hopf factorization.

-Virginia Polytechnic Institute and State Univirsity, Blacksburg. VA.

${ }^{\dagger}$ Texas Tech University Lubbock, TX.

\section{STATIONARY TRANSPORT WITH PARTIALLY REFLECTING BOUNDARY CONDTTIONS}

\author{
R. G. Cole $\quad$ T. Keyes" \\ V. Protopopescu
}

[Abstract of J. Chem. Phys. 81(6), 2771 (1984)]

The stationary monoenergetic transport equation (the Lorentz model) is considered in a semiinfinite geometry with partially reflecting boundary conditions at $x=0$. The extrapolation length, the density, and the space-dependent diffusion coefficient are studied as functions of the accommodation coefficient $\alpha$, both for constant and renormalized current. The hydrodynamical description at the level of the evolution equation is practically unaffected by $\alpha$ and is still valid at distances of several mean free paths from the boundary. Yet, when consistently applying the boundary condition, the boundary layer has to be considered in terms of the Milne extrapolation length, which varies as $(1-\alpha)^{-1}$. The case $\alpha=1$ is a singular limit in the sense that the boundary layer solution disappears completely.

- Boston University, Boston, MA.

2.61

\section{STATIONARY TRANSPORT WITH PARTIALLY REFLECTING BOUNDARY CONDITIONS. II.}

\section{R. G. Cole $\quad$ V. Protopopescu}

T. Keyes

[Abstract of J. Chem. Phys. (in preas)]

We consider the stationary monoenergetic transport equation ( the Lorentz model) in a semiinfinite geometry with most general nonmultiplying boundary conditions at the wall $x=0$, accounting for absorption, specular, diffuse, and "selective" reflection, or combinations thereof. 
Performing the numerical study for a (partially) thermalizing" wall, we investigate the density profile $n(x)$, the Milne extrapolation length $x_{M}$, and the space-dependent diffusion soefficient $D(x)$ as functions of the accommodation and "selection" coefficients. The inversed density profile - equivalent to a negative diffusion coefficient - is numerically computed. As a simple analytically solvable example, a discrete velocity model is discussed.

- Boston University, Boston MA

\section{ABSTRACT TIME-DEPENDENT TRANSPORT EQUATIONS*}

\section{R. Beals ${ }^{*} \quad$ V. Protopopescu}

[Abstract of J. Math. Anal. Appl. (in press)]

We consider the abstrac: time-dependent linear transport equation as an initial-boundary value evolution problem in the Banach spaces $\boldsymbol{L}_{p}$, $1 \leqslant p<\infty$, or in a space of measures on a (possibly time-dependent) kinetic phase space. Existence, uniqueness, dissipativity, and positivity results are proved for very general, possibly time-dependent, transport operators and boundary conditions. When the phase space, boundary conditions, and transport operator are independent of time, corresponding results are obtained for the associated semigroup.

- Work partially supported by the National Science Foundation. 'Yaic University. New Haven, $\mathrm{CT}$.

2.63

\section{THE ABSTRACT TIME-DEPENDENT TRANSPORT PROBLEM*}

\section{Protopopescu}

(Abstract of Int. Eqs. Oper. Theory (in press); also an invited lecture presented at the International Workshop on Operalor Theory and its Applications. Amsterdam. Holland, June 37 . 1985)

The generic abstract time-dependent transport equation is considered as an evolution (initial- boundary value) problem in the Banach spaces $L_{p}, 1 \leqslant p<\infty$, ani in the space of bounded measures. By using the trajectory method, the basic issues of existence, uniqueness, dissipativity, and positivity can be addressed and solved for very general, possibly time-dependent domains, operators, and boundary conditions.

- Research partially sponsored by U.S. Department of Energy.

\subsection{4}

\section{A SURVEY OF RECENT RESULTS AND OPEN PROBLEMS IN TIME-DEPENDENT TRANSPORT THEORY*}

\section{Protopopescu}

(Abstract of an invited lecture presented at the IX $X^{\text {th }}$ International Conference on Transpon Theory. Montecatini, Italy, June 10.14. 1985]

The state of the art in time-dependent lineas transport theory is reviewed. The generic abstract time-dependent transport equation is shown to be a well-posed evolution (initialboundary value) problem in the Banach spaces $L_{p}, 1 \leqslant p<\infty$, and in the space of bounded measures, for very general, possibly timedependent domains, operators, and boundary conditions. When the phase space, the transport operator, and the boundary cunditions are independent of time, the evolution problem can be solved by the usual semigroup method.

While existence and uniqueness are proved for essentially all situations of interest, the asympiotic behavior of the solution and the spectral decomposition of the transport operator are still aspects which could not be solved in full generality. Related open problems are discussed.

- Research partially sponsored by U.S. Department of Energy. 
Section 3

APPLIED PHYSICS AND FUSION REACTOR ANALYSIS

$10 / 6^{4}$ 


\title{
3.0. INTRODUCTION
}

\author{
R. G. Alsmiller, Jr.
}

\section{Fesion Reactor Research}

The division's magnetic fusion energy program includes integral experiments and their analyses and a va.iety of design calculations.

The integral experiments utilize a 14-MeV neutron source produced oy the interactions of 250-keV deuterons with tritium saturated in a titanium target. The experiments are designed to provide data against which ORNL and other organizstions involved in fusion reactor desiga can test transport methods and nuclear data. The principal design problems are associated with neutron streaming through the large ducts that are required in fusion reactor blankets and shields to accommodate neutral beam injectors, vacuum pumps, etc. As noted in papers included in this section, measurements have been made of $14-\mathrm{MeV}$ neutrons streaming through stainless steel ducts embedded in concrete, and the spectra of neutrons and gamma rays from these measurements have been compared with calculated results to test the adequacy of available design methods.

Other studies in the magnetic fusion energy program during this reporting period have consisted in calculations carrier out to aid in the design of particular devices such as the ELMO bumpy square.

\section{High-Energy Accelerntor Shiedting and Detector Research}

The division's calculations of the transport of high-energy particles continue to be performed at the request of groups outside of ORNL. With the methods and interaction data that have been developed and the experience that has been gained, calculations that were not possible a few years ago are now routinely performed at ORNL. A part of the program is also directed at improving the calculational methods and data that are available.

During this reporting period a variety of calculations have been carried out to aid in the design of instrumentation (ionization calorimeters) to measure the energy of various high-energy particles. Calorimeters, based in part on design calculations provided by the ORNL program, have been built and tested at accelerators and have been found to be satisfactory. The agreement of our calculations with the experimental tests is significant since the instruments are very large and expensive, and "iesting" design variations with a series of calculations rather than with numerous mockupe is much more economical. 


\section{Fusion Reactor Research}

3.1

\section{MONTE CARLO AND DISCRETE ORDINATES CALCULATIONS OF 14MEV NEUTRONS STREAMING THROUGI: A STAINLESS STEEL DUCT: COMPARISON WTTH EXPERIMENT I*}

\section{R. T. Santoro}

R. G. Akniller, Jr.
J. M. Barast

J. D. Drischler
(Abaract of ORNL/TM-9541, March 1985; awo Nud. Sci Eng. (in press); atso Sixth Topical Meeting on the Techoology of Fusion Energy. San Francisco, CA, March 3-7, 1985]

Measured and calculated neutron and gamma-ray energy spectra from $\sim 14-\mathrm{Me} J$ neutrons stre. ming through a stainless steel duct having a length-to-diameter ratio of 4.6 are compared. The 1.45 - $\mathrm{m}$-long duct is imbedded in a concrete block. The spectra were measured with an NE-213 liquid scintillator as a function of detector location relative to the mouth of the duct. The calculated data were obtained using the Monte Carlo code MCNP and the discrete ordinates code DOT 4.3. The calculations were performed using a two-dimensional cylindrical model of the experiment with symmetry about the duct axis. The measured and calculated neutron and gamma-ray spectra are compared at two distances from the mouth of the duct and at detector locations on and off the duct axis. The neutron spectra calculated with MCNP agree with the measured data within $\sim 5$ to $50 \%$ at all detector locations. The data calculated using the discrete ordinates method are in good agreement with the experiment for the cases where the detector is on axis but are in poor agreement at the off-axis detector locations. The gamma-ray spectra calculated with both radiation transport methods are in good agreement ( -5 to $25 \%$, depending on photon energy) with the measur i spectra.

:Research sponsored by U.S. DOE OTice of Fusion Enersy Computing and Telecommunications Division.
3.2

\section{CALCULATIONS TO EVALUATE THE USE OF THE ATTENUATION OF D-D NEUTRONS IN LIQUID OXYGEN AS A MEANS OF MEASURING PLASMA ION TEMPERATURE*}
R. G. Alwowler, Jr.
R. T. Santoro
J. F. Marneachenit $t$
J. M. Bancest [A hatrect of ORNL/TM-9230, July 1984; abo Fwsion Tech.
7. $197(1985)$ ]

The ion temperature of a deuterium plasma with a Maxwellian distribution can be determined by measuring the transmission of the deuteriumdeuterium (D-D) neutrons, i.e., neutrons produced by the reaction $\mathrm{D}+\mathrm{D} \rightarrow n+{ }^{3} \mathrm{He}$, tnrough liquid oxygen. In sractice the measurement requires both collimation and shielding to ensure that the attenuation of only those neutrons emitted directly from the plasma is measured. Calculated results are presented of the collimation and shiclding required to reduce the background so that the ion temperature may be measured. The geometric configuration used in the calculations is that of the Impurity Studies Experiment (ISX) at the Oak : ::dge National Laboratory, but the results will provide insight into the application of the measurement method at other plasma facilities. Results are presented for D-D plasma temperatures of 2,6, and $10 \mathrm{keV}$ and for two sizes of NE-213 detectors. It is concluded that the counting rates are too low to make the measurement feasible at ISX.

\footnotetext{
- Research sponsored by U.S. DOE OTice of Fusion Energy.

Computing and Telecommunications Division.
} 


\section{MULTIGROUP ENERGY-ANGLE DISTRIBUTIONS FOR NEUTRONS FROM THE T(d,n)tHe REACTION $\left(E_{d}=100-400 \mathrm{keV}\right)^{*}$ \\ R. T. Santero J. M. Barnest \\ J. D. Drisctiler R. G. Alomiler, Jr.}

(Abstrect of ORNL/TM-9251, July 1984)

The angle-energy dependence of neutrons produced in the $T(d, n)^{4} \mathrm{He}$ reaction from 100 - to 400-keV deuterons incicent on a $4-\mathrm{mg} / \mathrm{cm}^{2}$-thick titanium tritide target has been calculated.

-Recarch sponsoned by U.S. DOE Office of Fusion Energy. 'Computing and Tebocommunications Division.

3.4

\section{ORACT: A 174NELTRON-GROU: ACTIVATION CROSS-SECTION LIBRARY FOR FUSION AND FISSION REACTOR DESICN STUDIES•}

\section{R. T. Surtoro J. E. White \\ J. D. Drinchler \\ (Abatract of ORNL/TM-9203, Jupe 1989)}

A 174-neutron-group activation cross-section library for 233 ground-state target isotopes and 22 isomeric target isotopes is described. The library, ORACT, was deriva from the ACTL Evaluated Neutron Activation Cross-Section Library and was developed for use with existing computer codes that calculate induced activation by convoluting the cross-sestion data with neutron scalar flux distributions. The activation cross sections extend over the energy range frnm thermal to $20 \mathrm{MeV}$ and are useful for fusion and fission reactor nuclear design studies as well as other applications.

- Research sponsored by U.S. DOE Office of Fusion Energy.

\section{MAGNETICS CALCULATIONS FOR AN ELMO BUMPY SQUARE*}

\author{
R. T. Samtoro N. A. Ucton?
}

\author{
R. J. Setmint
} (Abatract of ORNL/TM-9542, March 1985; also Sixth
Topical Mecting on the Technology of Fusion Eneros. Stro
Francisco. CA. March 3-7. 1985]

Calculations have been carried out to determine the vacuum magnetic parameters, forces, and the use of trim coils in an ELMO Bumpy Square. A configuration having five mirror coils per side and an eight-coil high-field toroidal solenoid corner assembly was studied. Favorable magnetic parameters are achieved in the device. An on-axis mirror ratio of 1.9, a global mirror ratio of 3.6, and excellent centering of plasma pressure contours are achieved. Particle losses are also minimal ( $<5 \%)$. The magnetic forces acting between coils are comparable with those encountered in the EBTI/S magnet configuration. Circular trim coils were found to be suitable for restoring hot electron-ring locations that are displaced when the soil currents are vaiied for performing magnetic studies or for assessing the effects on the EBS of the global mirror ratio.

- Research sponsored by U.S. DOE OfTice of Fusion Enerty.

'Fusion Energy Division.

tMcDonnell Douglas Astronautics Company. St. Lowis, MO.

3.6

\section{AN EBT REACTOR SYSTEMS ANALYSIS AND COST CODE: DESCRIPTION AND USER'S GUIDE (VERSION 1)•}

\author{
R. T. Santoro \\ N. A. Uckn' \\ J. M. Barnest \\ D. E. Driemeyers \\ (Abstract of ORNL/TM-9026, June 1984)
}

An ELMO Bumpy Torus (EBT) reactor system analysis and cost code that incorporates 
the most recent advances in EBT physies bas been written. The code determines a set of reactors that fall with:n an a!lowed operating window determined from the coupling of ring and core plasma properties and the self-consistent treatment of the coupted ring-core stability and power balance requirements. The essential elements of the systems analysis and cost code are described, along with the calculational sequences leading to the specification of the reactor options and their associated costs. The input parameters, the constraints imposed upon them, and the operating range over which the code provides valid results are discussed. A sample problem and the interprctation of the results are also presented.

-Recarch sponsored by U.S. DOE OTtre of Fusing Energ:

Fusioa Energy Division

tCompatins and Telocommanications Divixion.

SMcDonell Douglas f stronautics Company, SI. Louia, MO

3.7

FUSION REACTOR SHIELDING BENCHMARK II. DUCT STREAMING EXPEAIMENTS AND ANALYSES*

G. T. Chapmant

R. G. Akriller, Jr.

J.S. Tand

R. T. Samtoro

J. M. Banest

P. D. Sornot

(Abstract of Shiclding Benchmark $S$ (SBS) in Cross Section Evaluation Working Group Benchmark Specifications. BNL. 19302 (ENDF-202). Vol. II, December 1983. P. F. Roxe and R. W. Roussin, Eds. !

Integral experiments that measure the streaming of $\sim 14-\mathrm{MeV}$ neutrons through a 0.30-m-diam cylindrical iron duct (length-todiameter $\sim$ ) have been carried out. The measured data were obtained using an NE-213 liquid scintillator detector with pulse-shape discrimination to separate neutron and gammaray events. Neutron (energy $\geqslant 850 \mathrm{keV}$ ) and gamma-ray (energy $\geqslant 750 \mathrm{keV}$ ) spectra have been measured at 16 locations on and off the axis of the duct. Spectra have been obtained when the cylindrical iron duct is partially filled by a laminated stainless steel and polyethylene shadow bar. Calculated results obtained using both discrete ordinates and Monte Carlo methods have been compared with the experimental spectra and favorable agreement (5-50\%) has been obtained.

:Research sponsored by US. DOE OTIx of Fusion Eacry.

'Rous- Stale Commuaity College. Harriman. TN.

tComputuri ud Telocommunicationas Division.

Schlumberiger Nell Servicas, Houston, Texas. 


\section{High-Energy Accelerator Shielding and Detector Research}

3.8

\section{THE HIGH-ENERGY TRANSPORT CODE, HETC}

\section{T. A. Galried}

(Abatrea of paper presented at the LEP Experimenters' Workehop on Shower Simulation, CERN, Geneva, Sritzerland, January 29-31, 1985)

The high-energy transport code :IETC has for many years been the benchmark code for hadronic transport calculations. Presented in this paper is a summary of the physics contained in HETC as well as some recently calculated data that are compared with experimental data.

- Research sponsored by U.S. DOE OfTice of High Energy and Nuckear Physia.

3.9

\section{COMPENSATION EFFECTS IN HADRON CALORIMETERS*}

\section{$\begin{array}{lll}\text { T. A. Galtrial } & \text { B. L. Butop }\end{array}$ \\ J. Brant A. Di Cinceiós \\ M. Goodene' R. Wituod}

(Abstract of paper presented at IEEE 1984 Nuclear Science Symposium, Orlando, FL, October 31-Nowembe - 2, 1984)

The pros and cons of utilizing a fissionable material such as ${ }^{238} U$ to compensate for the nuclear binding energy losses in a hadron calorimeter are discussed. Fissionable material can return some lost energy to the particle cascade in terms of low-energy neutrons and gamma rays, but electromagnetic sampling inefficiencies (often called transition effects) and the detection medium which tries to convert this energy to a useable signal are just as important.

- Renearch sponeored by U.S. DOE Office of High Enercy and Nuclear Phyzica.

Computing and Telecommunications Division.

tUniversity of Tennewce, Knoxville, TN.

SINFN/University of Roma, Roma, Italy.

Harvard University, Cambridge, MA.

3.10

\section{HADRON-LEPTON CASCADE \\ CALCULATIONS (1-20 GeV) FOR A PL-ALLUCTTE CALORMMTTIR*}

\author{
F. S. Alonerer T. A. Galviel \\ R. G. Alwoner, Jr.
}

[Abaract of ORNL/TM-9153, Angent 1984: sho Nuct. Instrum Methods in Phys. Reseerch A23, 175 (1985), also paper presented at the Soutbeastern Section Mecting of the American Physical Society. Memphin, TN, October 24-27. [984]

The results of Monte Carlo calculations for $\pi^{-}$(I to $20 \mathrm{GeV}$ ) and $e^{-}$(I to $10 \mathrm{GeV}$ ) normally incident on the center of a cylindrical calorimeter are presented. The calorimeter design has 60 unit cells of alternating $\mathrm{Pb}$ - $\mathrm{Al}$ Lucite plates and is a simplified model of one to be used at the Stanford Linear Accelerator Center. General characteristics of the total and spatial energy deposition are given. Pulse-beight spertra, as well as spectra of the transverse fractional energy distributions and of their first and second moments, are given to aid in hadronlepton discrimination. Sampling fluctuations are also examined for this purpose. Some results for the effects of a uniform probability of incidence over the central transverse strip and also some longitudinal discriminants are presented.

-Research sponsored by U.S. DOE OTice of High Enercy and Nuclear Pbyrica.

3.11

\section{MONTE CARLO STUDIES OF I'RANIUM CALORIMETRY*}

\section{J. Brant \\ T. A. Gabriel \\ B. L. Bieloopt \\ J. Hargist}

[Abatract of ORNL/TM-9486, Jenuary 1983; aleo Nucl. Insiru. Meihods A233, 489 (1985)]

Detailed Monte Carlo calculations of uranium calorimetry arc presented which reveal a 
significant difference in the esponses of liquid argon $a: d$ plastic scintillator in uranium calorimeters. Due to saturation effects, neutrons from the uranium are fuund to contribute only weakly to the liquid argon signal. Electromagnetic sampling inefficiencies are significant and contribute subsiantially to compersation in both systems.

-Research sponsorad by U.S. DOE OfIice of High Enerigy and Nuclear Physics.

University of Tennessee, Knoxvilk, TN.

fComponing and Telocommunications Division.

3.12

\section{A MONTE CARLO SIMULATIUN \\ OF THE RESPOVSE OF A \\ HADRONIC CALORIMETER \\ TO PROTONS OF MOMENTUM $3.5 \mathrm{TO} 200 \mathrm{GeV} / \mathrm{c}^{*}$}
A. I. Mincert
T. A. Gabriel
B. L. Bishopt
J. A. Goctenant
H. T. Freadeareicht
G. B. Yodh'
S. C. Toawart
R. W. Enswortb?

[Abstract of ORNL/TM-9270, January 1985; also Nucl. Instrum. Methods A238, 482 (1985)]

The response of scintillation counters in an ionization calorimeter to incident protons of momenta 3.5 to $200 \mathrm{GeV} / \mathrm{c}$ was simulated using the CALOR computer code system. Results of the simulation are compared with data taken at Brookhaven National Laboratory for 3.5- and $10-\mathrm{GeV} / \mathrm{c}$ protons and at Fermi National Accelerator Laboratory for 50-, 100, and 278.GeV hadrons. Mechanisms which produce large pulse heights for low-energy incident particles are discussed.

- Research sponsored by U.S. DOE Office of High Energy and Nuclear Physics.

University of Maryland, College Park, MD

tComputing and Telecommunications Division.

Fational Science Foundation. Washington, DC.

George Mason University, Fairfax, VA.
3.13

\section{MEASUREMENTS OF THE RESPONSE OF A HADRONIC CALORIMETER TO BEAMS OF PIONS AND PROTONS OF 1 TO 10 GEV MOMENTA*}
A. 1. Mincer'
H. T. Freadeareich'
J. A. Goodma'
G. B. Yout
S. C. Towrar"
R. W. Elsworths
D. Berkyt
B. L. Bistopt
T. A. Gabriel

[Abstract of Nucl. Instrum Methods (in press)]

An ionization calorimeter was studied with a test beam at the Brookhaven Alternating Gradient Synchrotron. The purpose of the experiment was to calibrate the response of a similar but larger cross section calorimeter used to study high-energy cosmic-ray showers a: the University of Maryland, College Park, Maryland. The beam meinenta were between 1 and 10 $\mathrm{GeV} / \mathrm{c}$. Results of measurements of (1) the mean pulse height from a scintillator at different depths in the calorimeter, (2) the frequency of occurrence of very rare events with pulse heights many times the mean pulse height, and (3) the correlation of pulse heights from several scintillators at different depths are presented and discussed.

- Research sponsored by U.S. DOE Office of High Energy and Nuclear Physics.

University of Maryland, College Park, Mr.

INational Science Foundation, Washington, DC.

${ }^{6}$ George Mason University, Fairfax, VA.

Computing and Telecommunications Division.

3.14

\section{AN INTRODUCTION TO COMBINATORIAL GEOMETRY*}

\section{T. A. Gabriel M. B. Emmett ${ }^{t}$}

(Abatract of paper presented at the LEP Experimenters' Workshop on Shower Simulation, CERN, Geneva, Switzerland, January 29-31, 1985)

The combinatorial geometry package which is useó with particle transport codes such as HETC, 
MORSE, and EGS is described from the user's point of view. The definitions of the various bodies used in the geometry package as well as the,+- , and/or operators used to combine these bodies are given and discussed. ieveral examples of simple geometries are provided.

- Research sponecred by U.S. DOE OITice of Hiph Ereng and Nuclear Physica.

Computing and Telocommunications Division.

\section{Energy Economies Modeling and Amalysis}

\subsection{5}

\section{A MODEL OF THE WORLD OL MARKET WITH AN OREC CARTEL*}

R. G. Akrmiller, Jr.

R. A. Marshaliat

\section{S. M. Hanst}

[Abetract of ORNI/TM-9204, August 1984; abo Energy 10, 1089 (1985)]

i world oil market model (WOM) with OPEC treated as a Stackelberg cartel has been developed within the framework of the Generalized Equilibrium Modeling System (GEMS) that is available from Decision Focus, Inc. The U.S. sector of the model is represented by a Liquid Fuels Supply model that was presented previously.

The WOM model is describad and results obtained with the model for the period 1980 to 2040 are presented. For comparative purposes, results obtained with the model when OPEC is treated as a competitive producer are also presented. By comparing the world oil price as a function of time from the two calculations, the influence that OPEC may have on the oil market by exploiting all of its market power is quantified.

The world oil price as obtained with the WOM model is also compared with world oil price projections from a variety of sources.

- Research sponsored by U.S. DOE former OTfice of Policy. Analysis, and System Studiea.

Computing and Telecommunications Division.

' Decision Focus, Isc., Los Alios, CA. 
Section 4

MATHEMATICAL MODELING AND INTELLIGENT CONTROL 


\subsection{INTRODUCTION}

\section{R. Weishin}

The work of this section continues to be divided into two major categories: (1) research on machine intelligence and advanced computer systems, and (2) mathematical modeling and performance assessment. The first category covers mainly the work performed in the Center for Engineering S:stems Advanced Research (CESAR), while the second category covers several areas in which anal/tic methods are developed and mathematical models are used to predict and evaluate the behavicr of dynamic systems. During this reporting period, this category has included calculations for light water reactor systems, radioactive waste disposal systems, atmospheric systems (the $\mathrm{CO}_{2}$ problem), and fossil energy systems.

\section{Artificial Intelligence, Robotics, and Adranced Competer Systems Research}

CESAR was established in November 1983 by the DOE Office of Basic Energy Sciences to perform research in the development of intelligent machines, i.e., artificially created operational systems capable of autonomous decision making and action. The center provides a framework for merging concepts of artificial and machine intelligence with advanced control theory, the overall goal being to move the human out of the control loop as far as possible so that systems can react more quickly, avoid potential mistakes resulting from operator fatigue, and minimize risk to humans involved in hazardous tasks. Already CESAR has conducted workshops in which some of the nation's foremost research leaders in robotics and artificial intelligence have participated, the most recent on planning and sensing for autonomous navigation [proceedings published as ORNL/TM-9923 (CESAR-86/01)]. In addition, CESAR's own research activities now include three activities sponsored by Army and Air Force Laboratories which complement the base DOE program in the areas of concurrent computation, dexterous manipulation in hazardous environments, and machine vision.

During these first two years, the research emphasis in CESAR has focused on autonomous remote operations that can be: applied in unstructured dangerous environments. In particular, the work has centered on the Jevelopment of self-powered robot prototypes, HERMIES-I and HERMIES-II, which contain many of the major fea: ires needed for remote work in hazardous environments. HERMIES-II consists of a wheel-driven chassis, dual manipulator arms, on-board distributed processors, and a clirectionally controlled sensor platform. A two-arm shoulder assembly has a total of 13 degrees of ireedom, and a sensory platform with stepper-motion-driven pan and tilt mechanism contains both ultrasonic ranging (phased arrays) and vision transducers to give the robot the ability to sense his world environment. Its position is currently controlled in an open-loop fashion by real-time monitoring of the wheel encoders and on/off control of the drive motors.

HERMIES-II has full cluplex communications with a dedicated LISP machine or with an NCUBE concurrent computer, which are used for algorithmic development of high-level reasoning in near-real-time task and path planning and for vision. The plans are implemented by returning 
executable command/control software primitives directly to the on-board distributed digital control system. Thus, the computers serve as an immobile brain" for the robot. Future HERMIES robots will have more advanced on-board brains.

Algorithms for obstacle avoidance have been successiully demonstrated with HERMIES-II, which recently has been upgraded with a phased array of sonar sensors and a new vision system. These navigation algorithms have been augmented with learning techniques which record and synthesize information from multiple journeys and which allow for continuous transition from local to global optimality in path planning.

A new research manipulator (CESARM) adapted from the Consolidated Fuel Reprocessing Program (CFRP) has been designed and built and now allows our analytical work on the modeling and control of flexible structures to be experimentally verified. The manipulator being developed weighs approximately $68 \mathrm{~kg}(150 \mathrm{lb})$ and can lift approximaiely $13.6 \mathrm{~kg}(30 \mathrm{lb})$, its weight-tocapacity ratio of approximately 5 being a factor of 4 improvement over typical industrial manipulators. Another scheduled addition to the robot will be a mobile platform more suited to navigation in unstrictured terrain.

Closely related to the HERMIES develcpment studies, and extending beyond them, is CESAR's work in the area of advanced computer systems. The advent of very large scale integratior. (VLSI) technology and its implernentation in the design of computer systems involving a high degree of concurrency is providing tremendous opportunities for a wide spectrum of applications which require "supercomputing" capabilities. The basic trend is to use state-of-the-art VLSI to integrate an entire processing system on a single chip, including communication links, memory interface, 32-bit processors, and even 64-bit IEEE floating point, resulting in smaller and cheaper processors comparable in performance to their larger and more expensive predecessors. This trend, which we see as continuing over the next decade, is the major technological drive behind concurrent computation, i.e., the use of an ensemble of small computers that work concurrently on parts of a complex problem and coordinate their computations entirely by sending messages to each other.

A computer design currently being investigated by CESAR is based on a hypercube architecture. Up to 1024 32-bit processors developed by NCUBE Corporation, each processor having the power of about one and a half VAX $11 / 780$ 's, can be connected to their nearest neighbors; however, sis.ce VLSI technology is used, the total physical volume of the NCUBE machine is much less than one cubic meter. The initial CESAR configuration is a six-dimensional cube, which became fully operational in January 1986.

Current research focuses on generalizing the simulated annealing global optimization methodology to systems with a varying number of degrees of freedom and on exploring its applicability to the static and dynamic load balancing of large-scale message-passing hypercube multiprocessors. Since the targeted applications involve hard real-time environments (e.g., robotics, SDI battle management, etc.), a significant effort is devoted to advanced operating system concepts such as "virtual time."

In addition, the development of parallel algorithms for machine vision is receiving increasing attention. In particular, work was initiated to study the applicability of human neural mechanisms for rohotic vision. Mathematical models were developed to describe the first three layers of the retina, and the models were evaluated by (1) using psychophysical experiments to parameterize the retinal equations and evaluate their consonance with human perception, and (2) deveioping a computer tool to simulate the dynamics of neural net interactions and their effects on input visual signals. 
Another area in which CESAR has been active is in developing a stronger thecretical basis for decision making under uncertainty than was available from existing analytical approaches. The result is a hybrid uncertainty theory which synthesizes and extends Dempster/Sbafer theory and fuzzy-set theory. The theory will be extensively tested in CESAR applications to plasning, sensor fusion, and rule-based systems once it has been reduced for efficient numerical implementation.

The long-term nature of the CESAR program may permit the "growth" and intellig-nce development" of the HERMIES robot series to be followed. While the SHAKEY robot system at Stanford Research Institute learned new operators for achieving goals, funding was such that considerations of memory never became an issue. Watching HERMIES grow would entail decisions regarding what to selectively forget (assuming finite memory constraints) and how to capture that elusive human property of common sense. We look toward real-time machine perseption from muttiple sensors; to high-speed, light-weight robots which exploit their flexible nature; to machines which can learn from experience; to coordinated multiple-arm (multiple finger) systems; etc. - all governed ultimately by Asimov's Three Laws of Robotics.

\section{Serveillance Dosinetry Andysis Methods for LWR Pressure Vesects}

Work on the LEPRICON computer code system - a complete methodology for analyzing dosimetry measurements to determine best estimates (with reduced uncertainties) of the neutron spectral fluxes in light water reactor pressure vessels - has continued and the methodology has now been successfully applied to an on-line reactor, Arkansas Nuclear One Unit 1 . On the basis of the results, an a priori lifetime some $50 \%$ longer than previous estimates is projected. After adjustment, the uncertainty in the projected lifetime was reduced by about a factor of two. (Previous estimates of the lifetime provided little if any uncertainty information.) Thus, it appears that Arkansas Power and Light can be provided with revenue for an additional period of 35 years with a defensible uncertainty of \pm 11 years. This projection is bzsed on a damage fluence of $2 \times 10^{19}$ neutrons $/ \mathrm{cm}^{2}$ above $1 \mathrm{MeV}$.

The development of the LEPRICON code system, which has been supported by the Electric Power Research Institute (EPRI), has been under way for several years and is described here in Papers 4.12 - 4.27.

\section{Reactor Physics Methods}

In the reactor physics area, we have concentrated on improving the reactor physics codes used by the utilities. During this reporting period our major accomplishment in this area has been to provide, improve, and document ENDF/B-V-based versions of the EPRI unit-cell and CPM assembly-lattice computer codes.

Our other research efforts have focused on theoretical improvements in the calculation of resonance absorption effects. Methods were developed to improve the calculation of neutron transmission probabilities in unit-cell interface-current codes (see paper 4.29), as well as to approximate interference factors.

\section{Performance Ascessment of Waste Isolation Systems}

Since the publication of the last progress report, a new program has been initialed within the division to assess th: performance of radioactive waste isolation systems. The objective of the program is to provide basic research support to the DOE Waste Management Programs and the Civilian Radioactive Waste Management Office. Emphasis in the initial phase of this program has 
been placed on the development of cost-efficient analytical and numerical methodologies for performing large-scale sensitivity and uncertainty analyses of complex design and modeling systems. Near-term applications of such methodologies include the design evaluation of a deep repository in salt, the performance assessrnent of high-level waste packagu and low-level waste isolation systems, and the evaluation of the efficiency of correcting existing waste disposal sites.

\section{Development of Amalytical and Automated Methods for Performance Assessment}

Our GRESS calculus precompiler is now being used for automated derivative generation supporting sensitivity analyses, mociel simplification studies and key parameter identification. Each Fortran line corresponding to a storage operation is analytically differentiated, and derivatives are propagated using the chain rule.

In the uncertainty analysis area, a new approact: (O-Thecry) has been developed to allow us to evaluate uncertainties in logical systems characterized by uncommitted belief and to quantify impiresision when using analysis trols such as rule-base 1 expert systems.

\section{Analysis of $\mathrm{CO}_{2}$ Impact on Climate}

The Carbon Dioxide Research Division of DOE brought the division's $\mathrm{CO}_{2}$ research program to an end because they peiceiver that climate models were not yet ready for the detailed sensitivity techniques that we were developing. Bringing the program to a graceful end was awkward because it had just reached the stage when detailed and accurate sensitivitiss were being produced for a general circulation model, but there werc not enough resources to demonstrate the value of these sensitivities in helping assess the validity of results. Nerertheless the effort concluded with (a) a position paper in DOE's state-of-the-art report on $\mathrm{CO}_{2}$ and climate which recommended how t.) apply uncertainty analysis to the $\mathrm{CO}_{2}$ problem, (b) a journal article showing how the adjoint method of sensitivity analysis can ie successfully applied to a general circulation model, and (c) a jrurnal article and technical note showing how the effect of various feedoacks (currently thrught to be one of th: greatest sources of uncertainty in climate models) can be isolated and efficiently estimated.

\section{Fossil Energy System3 Studies}

During this reporting period our work for the DOE/FE Office of Planning and Environment has focused on the replacement costs of U.S. domestic petroleum reserves and on the cost and competitiveness of advanced energy technologies. The methodology of the ORION code, which calculates costs versus cumulative supply of domestic oil on a regional basis, has been enhanced and the code has been utilized to assess the influence of economic criteria on estimates of U.S. offshore recoverable oil reserves. The Liquid Fuel Supply (LFS) model has been modified to allow much more exact representation of important input data (capital and operating costs, production data, etc.), and the results of the model have been compared with the 1985 National Energy Policy Plan (NEPP). A Replacement Cost Integration Program (RCIP) has been designed and coded for analysis of the replacement of U.S. oil reserves corresponding to domestic production, and the satisfying of the total U.S. demand for oil by the combination of imports and production from existing and previously undiscovered reserves. 
Since the publication of the last progress report, a new research program was also initiated with the DOE/FE Office of Oil, Gas, Shale and Coal Liquids (OGSCL). The objective of this program is to develop decision support systems to aid OGSCL in planning long-term, high-risk R\&D programs in the unconventional fuel technology area. In the initial part of the program our work has focused on the development of microcomputer models of world oil and gas markets that can be used to simulate market penetration of unconventioral fuels under widely varying economic and energy demand scenarios. (Note: Published work in this area during this reporting period is cited in paper 3.15 of this report.) 


\section{Artificial Intelligence, Robotics, and Adranced Computer Systems Research}

4.1

\section{ARTIFICIAL INTEILIGENCE AND CONCURRENT COMPUTATION FOR ROBOTIC APPLICATIONS*}

\author{
C. R. Weisbin \\ J. Bartea \\ G. de Sansoure \\ C. Jorgensen \\ E. M. Oblow \\ W. R. Hanelt \\ J. L. Laciust \\ T. Swift ${ }^{t}$
}

IAbstract of Trans. Am Nucl. Soc. 49, 310 (1985); also paper presented at the 1985 WATTec Conierence, Knoxville. TN. February 12, 1985]

The Center for Engineering Systems Advanced Research (CESAR) is currintly using the HERMIES-I nobile robot as an experimental focal point for research in artificial intelligence. Specific investigations underway include research in man-machine communication; planning and replanning under execution, including sensor feedback; and machine perception of fuzzy images. Investigations are focussed through design and execution of a series of increasingly complex tasks.

This paper describes current updates of HERMIES-I, including a recently mounted pair of 6 degree of freedom arms, full duplex communication between the multiprocessor system on board HERMIES-1 and a user host computer (e.g., LISP machine, IBM PC/XT, etc.), and current documentation. We discuss our initial navigation experiment simulating an unstructured world with moving obstacles. The "world" seen by HERMIES-I is displayed on an auxiliary graphic simulator on the host LISP machine, where high-level reasoning is performed when necessary and instructions are transmitted back to HERMIES-I for execution. Difficulties introduced through inadequate resolution of sensors call for a methodology for decision-making under uncertainty. An integration and extension of Dempster-Shafer Theory and the Theory of Fuzzy Sets is proposed. Concepts of associative memory are also being explored for the integration of information from two different sensors.

Finally, we are examining the LMI PICON system structure to invertigate potential hierarchical task decomposition between high level reasoning and numerical computation. PICON directs data acquisition and processing by a Motorola 68010 from inference performed in parallel using a LISP processor which can recognize important conditions and perform diagnosis.

Emphasis on real-time operation imposes a heavy computational burden which we are now addressing through procurement of a powerful very large scale integration (VLSI) concurrent computation machine with hypercube topology. The capacity and portability of the machine makes it particularly attractive for mobile applications. Implementation of virtual time concepts is being studied as the framework for a real-time robot operating system.

- Research sponsored by U.S. DOE Office of Basic Energy Sciences.

$t_{\text {Instrumentation and Controls Division. }}$

${ }^{t}$ Computing and Telecommunications Division.

4.2

\section{CENTER FOR ENGINEERING SYSTEMS ADVANCED RESEARCH PROGRAM*}

\section{R. Weisbin $\quad$ K. McKeebnat}

(Abatract of Brochure, February 1985)

This brochure provides an overview of the Center for Engineering Systems Advanced Research (CESAR). It describes the CESAR char er, research objectives, staffing, facilities, advisory committee, etc., as of February 1985. The brochure has been distributed within the machine intelligence and advanced control communities nationwide.

- Research sponeored by U.S. DOE Ofirce of Basic Enersy Sriences.

Information Resourcea Division. 
4.3

\section{MINIMAL CUT-SET METHODOLOGY FOR ARTIFICIAL INTTLUIGENCE APPLICATIONS*}

\author{
C. R. Weisher \\ G. de Sansoure \\ J. Bartea \\ E. M. ONow \\ J. C. White't
}

IAbstract of paper presented at First Conference on Artificial Intelligence Applications, Denver, CO, December 5-7. i984; Proc. p. 465 (1985)]

This paper reviews minimal cut-set theory and illustrates its application with an example. The minimal cut-set approach uses disjunctive normal form in Boolean algebra and various Boolean operators to simplify very complicated tree structures composed of AND/OR gates. The simplification process is automated and performed off-line using existing computer codes to implement the Boolean reduction on the finite but large tree structure. With this approach, on-line expert diagnostic systems, whose response times are critical, could determine directly whether a goal is achievable by comparing the actual system state to a concisely stored set of preprocessed critical state elements.

- Research sponsored by U.S. DOE Office of Basic Energy Sciences.

${ }^{t}$ Birmingham-Southern College, Birmingham, AL.

4.4

\section{AUTOMATED PLANNING FOR INTELLIGENT MACHINES IN ENERGY-RELATED APPLICATIONS*}

\section{R. Weitabir G. de Samaure}

\section{J. Bartea}

(Abaract of paper presented at the 1984 Confecence on Intelligent Systems and Machines, Oakland Univeni'y, Rocheater, MI, April 24-25, 1984)

This paper discusses the current activities of the Center for Engineering Systems Advanced Research (CESAR) program relatal to plan generation and execution by an intelligent machine.
The system architecture for the CESAR mobile robot (named HERMIES-I) is described. The minimal cut-set approach is developed to reduce the tree search time of conventional backward chaining planning techniques. Finally, a realtime concept of an Intelligent Machine Operating System is presented in which planning and reasoning is embedded in a system for resource allocation and process management.

-Research sponsored by U.S. DOE OTIxe of Basic Energy Sciences.

\section{5}

\section{REAL-TIME PLANNING BY AN INTELLIGENT ROBOT*}

\author{
J. Bartien C. R. Weishin \\ G. de Samaire
}

(Abstract of paper preaented at ASME Conference on Contputers in Engineering. Las Vegas, NV, Auguat 1984)

The development of automated decision making capabilities for an autonomous intelligent machine operatitig in dynamic real-world environments is one of the key research activities currently carried out within the Center for Engineering Systems Advanced Research at the Oak Ridge National Laboratory. The conceptual design of a Robot Operating System is presented as a basis for further research in the area of real-time planning. The Robot Operating System controls and directs the robot's planning and reasoning capabilities by embedding them, as asynchronous parallel algorithms, within a multiprocessor operating system built for resource allocation, process (e.g., sensors, arms, ...) manage ment, etc. The Center for Engineering Systems Advanced Research HERMIES-type robots provide the experimental focus for this activity.

- Recearch sponsored by U.S. DOE OfTice of Bacic Enerty Sciences. 
4.6

\section{MACHINE INTELLIGENCE FOR ROBOTICS APPLICATIONS*}

\author{
C. R. Weistic J. Bartea \\ G. de Sawsere W. R. Haned \\ C. C. Jorgensea E. M. Oston \\ R. E. Ricks
}

(Abarace of paper presented at the 1985 Conference on Intelligent Systems and Machines. Oakland University, Rocheater. MI, April 22-24, 1985)

The purpose of this paper is to review research in machine intelligence ongoing at the Center for Engineering Systems Advanced Kesearch (CESAR). As a result of initial experimentation with our HERMIES-I mobile robot, hardware and software upgrades were implemented which enable fully asynchronous sonar operation, improved stepper motor contro' for the sensory platform, and more reliable wheel drive control. Section II discusses the current system, dinignatrd as HERMIES-11. Section III reports successful demonstration of deadreckoning navigation and the development of a sensor-based exploration and discovery algorithm which can now handle typical maze problems. The development of HERMIES" "brain" as a hypercube ensemble machine with concurrent computation and associated message passing is described in Section IV. Algorithms for mapping precedence-constrained task graphs onto a hypercube yield results with high efficiency and proper load balance. Finaily, Section $V$ describes a framework for a hybrid uncertainty analysis theory for decision makıng.

-Research sponsored by U.S. DOE OTIice of Busic Energy Sciences.

IInstrumentation and Controls Division.
4.7

\section{HERMIISS-I: A MOBILE ROBOT FOR NAVIGATION AND MANIPULATION EXPERIMENTS*}

\author{
C. R. Weistiv \\ J. Bartwea \\ G. de Sansarere \\ C. Jorgensea \\ W. R. Hanel ${ }^{t}$ \\ E. M. Ostow \\ J. L. Laciest \\ T. Swift
}

(Abstract of paper presented at Robots 9 Conference, Detroit, MI, June 2-6, 1985)

The purpose of this paper is to report the current status of investigations ongoing at the Center for Engineering Systems Advanced Research (CESAR) in the areas of navigation and manipulation in unst ructured environments. Section II discusses the HERMIES-I mobile robot, a prototype of a series which contains many of the major features needed for remote work in hazardous environments. Initial experimental work at CESAR has begun in the area of navigation. Section III briefly reviews some of the ongoing research in autonomous navigation and describes initial research with HERMIES-I and associated graphic simulation. Since the HERMIES robots will generally bc composed of a variety of asynchronously controlled hardware components (such as manipulator arms, digital image sensnrs, sonars, etc.), it seems appropriate to consider future development of the HERMIES "brain" as a hypercube ensemble machine with concurrent computation and associated message passing. The basic properties of such a hypercube architecture is presented in Section IV. Decision-making under uncertainty eventually permeates all of our work. Following a survey of existing analytical approaches, it was decided that a stronger theoretical basis is required. As such, Section $V$ presents the framework for a recently developed hybrid uncertainty theory.

-Revearch sponeored by U.S. DOE Office of Basic Energy Sciences.

Instrumentation and Controls Division.

tComputing and Telocommunications Division. 
4.8

\section{HYPERCUBE CONCURRENT COMPUTATION AND VIRTUAL TIME FOR ROBOTIC APPLICATION*}

\section{J. Bartea}

(Abatract of paper presented at the IEEE Wortabop on Special Compriter Architectures for Robotica and Automation, International Conference on Roioxic Research, St. Louis, MO, March 25, 1985)

The programmatic activities of CESAR, the Center ior Engineering Systems Advanced Research, are briefly reviewed. In the concurrent computation area, critical issues relatzd to the control of intelligent machines using hypercube ensembles are outlined. The mapping of task graphs exhibiting structural irregularity and nonlocal communication patterns is discussed and the results of an application to the solution of robot inverse dynamics are given. Finally, the design of a Virtual Time Intelligent Machine Operating System (IMOS/VT) for the ORNL/NCube ensemble computer is presented.

-Research sponsored by U.S. DOE Office of Basic Energy Sciences.

\section{9}

\section{ROBOT INVERSE DYNAMICS ON A CONCURRENT COMPUTATION ENSEMBLE*}

\section{J. Barben}

(Abatract of nar. presented at lis 1985 ASME In'ernational Conference a Computers in Enginoering. Baston, MA, August 4 6, 1985)

Advances in VLSI technology provide a strong incentive to configure the "brain" of future intelligent robots as concurrent computation ensembles. The design of real-time operating systems for such machines is an important objective of the CESAR program. This paper addresses the development of ROSES, a prototype software system intended to provide expert scheduling capabilities in mapping precedence-constrained task graphs onto a computation ensemble. This problem being strongly NP-complete, ROSES attempts to achieve near-optimal solutions by combining heuristic techniques (to handle time complexity) with special instances of abstract data structures (to handle space complexity). The technique is applied to the solution of the inverse dynamics equations of a robot arm and arhieves high efficiency and proper load balance. Criscal concepts in the longer-term development of a Virtual Time Intelligent Machine Operating System (IMOS/VT) are also discussed.

-Research sponsored by U.S. DOE Office of Basic Energy Sciences.

4.10

\section{CONTROL OF A SINGLE LINK, TWO- DEGREE-OF-FREEDOM MANIPUI ATOR WITH JOINT COMPLLNCE AND ACTUATOR DYNAMICS*}
M. G. Forrest-Bartact
S. M. Babcoett
H. Singhs
M. J. Ralines

(Abatract of paper presented at the 1985 ASME Isternational Conference on Computers in Engineering, Bodon, MA, Auguat (-8, 1985)

One problem which limits the cycle time of typical industrial manipulators is the vibration which results if the modes of vibration of a manipulator with significant joint compliance are excited. In this paper the effects of joint compliance in a two-degree-of-freedom manipulator are modelled and a study of several possible controller structures is conducted for the problem of end effector position control. Controller structures considered include independent joint control neglecing compliance, independent joint control including actuator dynamics and joint compliance, inverse dynamics, and a deccupling con. troller for a model linearized about an arbitrary trajectory. Control law synthesis is performed utilizing REDUCE, a LISP-based program for symbolic aigebra. Simulation of various controllers is performed utilizing Advanced Continuous Simulation Language (ACSL). It is shown that 
the closed-loop-frequency response of the manipulator can be increased beyond the typical industrial limit of $50 \%$ of the manipulator's natural frequency while maintaining optimal response characteristics by considering the joint compliance in the design and providing suitable feedback measurements.

-Research sponsored by U.S. DOE OfTree of Baxic Enerty Sciences.

'Ph.D. Sudenth Wayoe State Univerrity, and Summar

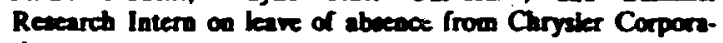
tion.

I Imatrumentation and Controls Division.

fWayne State University, Detroit. MI.
4.11

\section{A HYBRID UNCTRTAINTY THEORY*}

\author{
E. M. OAlon
}

(Abatrect of paper preseatod of the Intersational Wortubop oe Expen Systems and Their Appications - Palnis de Congres - Avigen, France, May 13-15, 1985)

A hybrid uncertainty theory for artificial intelligence problems combining the strengths of fuzzy-set theory and Dempster/Shafer theory is presented. The basic operations for combining uncertain information are given with an indication of their applicability in expert systems and robot planning problems.

-Research sposwore' by U.S. DOE Ofifice of Basic Enerty Sciencea

\section{Development and Application of LWR Pressure Vessel Surveiliance Dosimetry Analysis Methods}

\subsection{2}

\section{ACTIVITY AND FLUENCE CALCULATIONS FOR THE STARTUP AND TWQ-YEAR IRRADIATION EXPERIMENTS PERFORMED AT THE POOLSIDE FACTLTYY}

\section{$\begin{array}{ll}\text { R. E. Maerter } & \text { B. A. Worky }\end{array}$}

[Abstract of ORNL/TM-9265 (NUREG/CR-3886), October 1984]

Fluence rate, fluence, and activity calculations are presented for each of three exposures (two surveillance capsules and a pressure vessel capsule) performed during the two-year metallurgical blind test experiment at the ORR-Poolside Facility in Oak Ridge. This experiment is intended to serve as an international metallurgical benchmark, and the spectral fluence calculations described in this report have been made available to the international community in pressure vessel dosimetry to be used in their damage assessment studies and/or adjustment procedurus.

Following the same simplified calculational methods introduced and described in a reanalysis of the startup experiment, fission source distributions were obtained from three-dimensional diffu- sion theory for most of the 52 cycles active during the course of the complete experiment, combined in small groups, and the resultant ex-core group fluxes calculated by two-dimensional discrete ordinate transport theory. Dosimeter activities at the cnd of each of the three irradiations were calculated by decaying the saturated activities obtained for each group of cycles and summing over the pertinent cycles. Calculations indicated that cycle-to-cycle variations of the saturated activities could be as much as $40 \%$.

Comparisons of the dosimeter end-ofirradiation activities with HEDL measurements indicate agreement generally within $15 \%$ for the first surveillance capsule, $5 \%$ for the second, and $10 \%$ for three locations in the pressure vessel capsule, which are as good as if not somewhat better than comparisons in the startup experiment. The calculations thus validate the trend of the measurements in both the startup and the two-year experim.ents, and confirm the presence of a significant cycle-to-cycle variation in the core leakage. The tape containing the unadjusted spectral fluences for each of the three exposures that can be used in the metallurgical analysis is thus considered to be accurate to within about $10 \%$.

- Research sponeored by U.S. Nuctear Regulatory Commisaio.. 


\section{CALCULATTD SPECTRAL FLUENCTS AND DOSIMITTER ACTIVITIES FOR THE METALLURGICAL BLIND TEST IRRADIATIONS AT THE ORR-PSF*}

\author{
R. E. Maerter $\quad$ B. A. Worky
}

[Abstrace of peper preserted at the Fifth ASTM-Euratom Sympsium on Reactor Dosimetry, Geasthacht, Weat Germany. September 24-28, 1984; Proc. Vol. 1, p. 187, Dordrecht, Netherlands (1985)]

Fiuence rate, fluence, and activity calculations were performed for each of the three exposures (two surveillance capsules and a pressure vecesel capsule) performed during the two-year metallurgical blind test experiment at the ORRPoolsice Facility in Oak Ridge. Motivation for these calculations was prompted by differences of up to :5\% between dosimetry measurements performed in the earlier startup scoping experiment and the two-year experiment.

Comparisons of the dosimeter end-ofirradiation activities with HEDL measurements indicate agreement generally within $15 \%$ for the first surveillance capsule, 5\% for the second, and $10 \%$ for three locations in the pressure vessel capsule, which are as good as (if not somewhat better than) comparisons in the startup experiment. The ..lculations thus validate the trend of the mea srements in both the startup and the two-year experiments and confirm the presence of a significant cycle-to-cycle variation in the core leakage. The tape containing the unadjusted spectral fluences for each of the three exposures that can be used in the metallurgical analysis is thus considered to be accurate to within about $10 \%$.

\footnotetext{
- Research sponeored by the Nuclear Regulatory Commiation and U.S. DOE Surveillance Docimetry Improvement Program.
}

\section{NEUTRON CALCULATIONS OF THE 1WO- YEAR IRRADIATION EXPERIMENT AT THE ORR-PSF AND COMPARISON WITH MEASUREMENTS*}

\author{
R. E. Meerter $\quad$ B. A. Worky
}

[Abatrece of Trass. Am. Nuch. Soc 4634 (1984)]

Flux, fluence, and dosimeter activities were calculated for the 2-yr metallurgical blind test irradiation experiment performed at the Oak Ridge Reactor-Poolside Facility (PSF) during the period from April 1980 to June 1982. This experiment is intended to serve as an international benchmark to evaluate the accuracy of damage predictions in pressurized water reactor pressure vessels.

Early in the calculations it became apparent that significant cycle-to-cycle variations could exist in the core leakages among the 52 cycles active in the irradiation, so that in order to compare the dosimetry calculations with measurements, few shortcuts could be employed and nothing short of a complete analysis taking into consideration the source distribution of each of the 52 cycles as well as their leakages would suffice if an accurate comparison were desired. The calculations involved use of the three-dimensional difusion code VENTURE for the criticality and source distribution calculations, the two dimensional discrete ordinates transport code DOT4, and the one-dimensional discrete ordinates transport code ANISN for the flux calculations, and several other special purpose codes written to manipulate and combine the calculated data. The end result of these calculations was the generation of a tape that contains spectral fluence information for all the locations in the two simulated surveillance capsules, the pressure vessel 
capsule, and the void box capsule at which the metallurgical specimens were irradiated. Along the way, comparisons were made with dosimetry measurements performed throughout the experiment by the Hanford Engincering Development Laboratory (HEDi).

From all these dosimetry comparisons, it is expected that the calculated spoctral fluences on which the metallurgical analyses will be based should be accurate to within $\sim 10 \%$.

- Research sponsored by U.S. Nuclear Regulatory Commiscion Office of Nuctear Regulatory Research.

\subsection{5}

\section{GAMMA-RAY CHARACTERIZATION OF THE TWO-YEAR IRRADIATION EXPERIMENT PERFORMED AT THE POOLSIDE FACILITY*}

\section{R. E. Maerker}

[Ab-tract of ORNL/TM-9440 (NUREG/CR-4039), January 1985]

Average gamma-ray group fluence rates are calculated for each of the three exposures in the two-year metallurgical blind test experiment at the ORR-Poolside Facility in Oak Ridge, thus completing the characterization of the radiation field for this experiment, which is intended to serve as an international metallurgical benchmark. Heating rates in the steel derived from th.ese calculations varied from about 0.23 watts/gram in the simulated surveillance capsule to 1.4 milliwatts/gram at the three-quarters depth location in the simulated pressure vessel capsule, with secondaries arising from non-fission reactions in the core and ex-core steel contributing between 77 and 93 percent of the total. Contributions from photofission to fission toil activities are estimated to be less than five percent of those previously calculated arising from neutroninduced fission.

- Recearch sposusored by U.S. Nuclear Regulatory Commission Division of Engineering Technology.
4.16

\section{VALIDATION OF NEUTRON TRANSPORT CALCULATIONS IN BENCHMARK FACILITIES FOR IMPROVED DAMAGE FLUENCE PREDICTIONS*}

\author{
M. L. Willians ${ }^{t}$ \\ F. W. Stallmant \\ R. E. Maerter \\ F. B. K. Kant
}

[Abstract of Nuel. Eng. Design 86, 87 (1985)]

Neutron transport calcrlations play a vital role in the determination of the amount of radiation damage accumulated by reactor pressure vessels, and hence are an important factor in establishing reactor lifetime. Benchmark experiments are required to evaluate the validity and limitations of transport calculations. A comprehensive benchmark program is currently supported by the US NRC and several European organizations to test various aspects of the overall fluence calculational procedure. This paper summarizes the needs and - fuirements of the overall benchmark program. Results and conclusions of some current benchmark experiments are reviewed. Finally, some approximations in transport calculations which need validation, but which are not addressed in current benchmark efforts, are discussed.

- Rescarch sponsored by the U.S. Nuclear Regulatory Commission. Office of Nuclear Regulatory Research.

'Louisiana State University, Baton Rouge, LA.

Operations Division.

4.17

THEORY OF A NEW UNFOLDING PROCEDURE IN PWR PRESSURE VESSEL DOSIMETRY AND DEVELOPMENT OF AN ASSOCIATED BENCHMARK DATABASE*

\section{$\begin{array}{ll}\text { R. E. Maerker } & \text { B. L. Broadbead }\end{array}$ J. J. Wagachaft}

[Abstract of Nucl. Sci. Eng. 91, 369 (1985)]

The theory of a new methodology for quantifying and then reducing the uncertainties in the pressure vessel fluences (or fluxes) of a PWR is 
described. The theory involves combining the results of calculated and measured dosimetry integral experiments along with differential data used in the calculations, together with covariances, into a generalized linear least-squares adjustment code named LEPRICON. The procedure solves the translation problem necessitated by the use of ex-situ PWR dosimetry, and its covariance reducing potential is further enhanced by simultancously combining the PWR data with a database consisting of the results of analysis of simpler benchmark experiments. Development of this database and a demonstration of the uncertainty reduction with application to one of the benchmark eiperiments are also described. For the example chosen, covariances of the calculated fluxes were reduced by factors of between four and eight.

-Reacarcb sponsored by the Electric Power Researeb Institute. Palo Alto, CA.

${ }^{t}$ Computing and Telecommunications Livision.

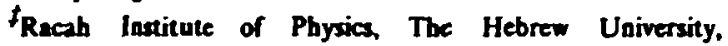
Jerusalem, lsraed.

4.18

\section{REVISION AND EXPANSION \\ OF THE DATA BASE IN THE LEPRICON DOSIMETRY METHODOLOGY *}

\section{R. E. Maerter M. L. Willians' \\ B. L. Broadleadt J. J. Wapactats}

C. Y. Fu

(Abstract of EPRI NP-3841, January 1985)

The objective of this ongoing study is to develop and use an advanced methodology for reducing the uncertainties in estimates of the spectrum and total fluence in a pressure vessel of an LWR from analysis of dosimetry measurements performed at a surveillance position. Ultimately, the success of the study will be measured in terms of increased lifetimes of existing reactors which are limited by embrittlement of the pressure vessel steel.

The methodology involves combining differential and integral dosimetry data, both measured and calculated, in a consistent fashion from various benchmark fields of increasing complexity, eventually including fields of operating power reactors. Covariances of all the data as well as sensitivities of the integral data to the differential data are required as a precondition to a meaningful combining process.

This new methodology (code-named LEPRICUN) has the power to supplant existing methods of spectral unfolding because it can be used to obtain not only better estimates of the fluence and spectrum in the pressure vessel but also of the uncertainties in these quantities, and these uncertainties are always less than those using existing metinods.

This report is the second in a series of interim reports demonstrating such an uncertainty reduction. It descrits the completion of the analysis of thirty-seven measurements performed in four fields of high diversity whose purpose is to serve as dosimetry benchmarks. It is intended that LWR surveillance data will be simultaneously adjusted with thece benchmark measurements to yield unfolded spectral fluences of high accuracy in the pressure vessel.

- Research sponsored by Electric Power Recarch Institula

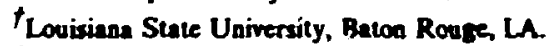

tComputing and Telecommunications Division.

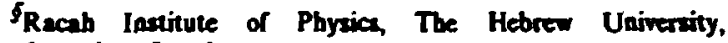
Jerusalen, Israel.

\subsection{9}

\section{THE ELXSIR CROSSSECTION LIBRARY FOR LWR PRESSURE VESSEL IRRADIATION STUDIES: PART OF THE LEPRICON COMPUTER CODE SYSTEM*}
R. E. Maerter
W. E. Forl, III ${ }^{t}$
C. C. Wobuter'
M. L. Willinent

(Abatract of EPRI NP-3654, September 1984)

A multigroup cross-section library named ELXSIR has been developed for use by utilitiea in computing the fluence to pressure veasels of light water reactors. The library is applicable to 
transport calculations performed with the DOTIV code, which is used as part of the LEPRICON code system developed through EPRI. Characteristics of the library, as well as the procedures employed for its generation, are described in this report.

- Research sponsored by Electric Forrer Research Institute.

'Compating and Telecommunications Division.

ILovisiana State University, Batoo Rouge, LA.

\subsection{0}

\section{UNFOLDED ANO-1 FLUXES USING THE LEPRICON METHODOLOGY*}

\section{J. J. Wasschalt \\ R. E. Meerter \\ B. L. Brondheadt \\ M. L. Wilinings}

[Abatract of payer presented at the Fifth ASTM-Euratom Symposium on Reactor Dosimetry, Geesthacht. West Germany, September 24-28, 1984; Proc. Vol. I, p. 1827, Dordrecht, Netherlands (1985)]

Calculated fluxes at a one-quarter depth midplane location in the pressure vessel of the ANOI reactor were adjusted using the LEPRICON methodolog;'. The adjusiment was based on the LEPRICON benchmark database in combination with the ANO-1 cavity activation measuremenis. The LEPRICON database has been expanded and now also includes the PSF field, of which the analysis has been recently completed. The measured, calculated, and adjusted ANO-1 activations are presented together with their corresponding uncertainties. However, the main purpose of this work is to obtain more credible flux values in the pressure vessel. The pressure-vessel flux adjustments and the reduction in the pressure vessel flux uncertainties are elaborated on in the concluding section.

\footnotetext{
- Recarch sponsorod by Electric Power Research Institute.

Racah Institute of Physica. The Hebrew University, Jerusalem, Israel.

tComputing and Telecommunications Division.

Lovisiana State University, Baton Rouge, LA
}

4.21

\section{LEPRICON - A SYSTEMATIC APPROACH TO LWR PRESSURE VESSEL DOSIMETRY*}

\author{
O. Ozert \\ R. E. Maerter \\ M. L. Willianst \\ B. L. Broadread \\ J. J. Wagectur \\ C. Y. F
}

[Abstract of paper presented at the ANS Topical Conference on Reactor Physics and Shielding, Chicago, Illinois, September 17-19, 1984; Proc. Vol. II, p. 674 (1984)]

A pressure vessel dosimetiy methodology based on a generalized least squares approach for adjusting calculated neutron fluxes and reaction rates on the basis of information from foil activation measurements is described. This methodology. incorporated into a computer code system called LEPRICON, permits adjustments to be carried out simultaneously for a series of benchmark fields as well as a reactor field of interest, thus insuring the consistency of the adjusted reactor fluxes with benchmark data. Development of a benchmark experiment data base for use in the analysis is described. Results obtained in the Arkansas Nuclear One-Unit 1 reactor are discussed.

\footnotetext{
- Revearch sponeored by Eleceric Power Research Institule.

IElectric Power Revearch Institute.

Louisiana State University, Baton Rouge, LA

${ }^{5}$ Computing and Telocommunications Division.

Racah Institute of Physics. The Hebrew University. Jerusalem, Israel.
} 
COMBINING INTEGRAL AND DIFFERENTIAL DOSIMETRY DATA IN AN UNFOLDING PROCEDURE WTTH APPLICATION TO THE ARKANSAS NUCIEAR ONE-UNIT 1 REACTCR*

\author{
R. E. Maerter \\ C. Y. Fu \\ B. L. Bromiluead \\ J. J. Wagachatt \\ J. Wintans M. L. Writhord
}

(Abatract of paper presented at the Interatioal Confercuoce on Nuctear Dall for Basic and Applied Sciences, Santa Fe, NM, May 13-17, 1985)

The LEPRICON adjustment procedure involves combining both differential and integral data, including covariances and sensitivities, in such a way that calculated spectral fluences at important locations within a pressure vessel of an operating PWR can be adjusted with significantly reduced uncertainties. The procedure allows simultaneous combination of integral dosimetry measurements performed at a reactor surveillance location vith measurements performed in geometrically simpler benchnark facilities. An application of this technique is given to an existing PWR, and the results o.nsistently indicate a need for significant ( -8\%) adjustments in the total inelastic cross section of iron in the region between 3 and $8 \mathrm{MeV}$ using cross sections and covariances from ENDF/B-V. Dosimetry cross sections were taken mainly fr $\mathrm{m}$ a revised version of ENDF/B-V and were found to require relatively small adjustments. Covariances of the spectral fluences are reduced by factors lying between two and four.

- Recarch sponsored by the Electric I'ower Research Institute.

'Computing and Telocommunications Division.

'Racah Institute of Physics. The Hebrew University. Jerusalem. Israel.

SUniversity of Arkanses, Fayetteville, AK.

Pouisiana State University. Baton R.wuge, LA.

\section{APPLCATION OF LEPRICON METHODOLOGY TO THE UNFOLDNG OF NEUTRON FLUXISS IN THE ANO-1 REACTOR*}

\author{
$\begin{array}{ll}\text { R. E. Merter } & \text { B. L. Brenineadt }\end{array}$ \\ M. L. Wringt
}

[Abatract of Trans. Am Nucl. Soc. 4, 425 (1985)]

The latest development of the LEPRICON methodology is the completion of the dosimetry benchmark data base with a subsequent application of the entire system to a analysis of measurements in an existing power reactor to gange its efficacy in a iealistic situation.

The result of this application to the ANO-I reactor was a reduction in the covariance of the accumulated pressure vessel fluence by about a factor of 2.5. This corresponds to a decrease in the standard deviation of the lifetime, based on a limiting fluence above $1 \mathrm{MeV}$ of $2 \times 10^{19} \mathrm{n} / \mathrm{cm}^{2}$, from 22 to 11 effective full-power years. The estimated lifetime, based on the same criterion, has been increased from 92 to 129 effertive fullpower years.

- Research sponsored by Electric Power Remearch Inatitute

'Computing and Telocommunications Division.

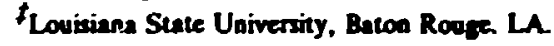


4.24

\section{APPLICATION OF THE LEPRICON UNFOLDING PROCEDURE TO THE ARKANSAS NUCLEAR ONE-UNIT 1 REACTOR*}

\section{R. E. Merker \\ B. A. Wortey \\ B. L. Broedlleadt \\ M. L. Wilinanst \\ J. J. Wagachaf}

[Abstract of Nucl. Sci Eng. (in press)]

This is the second of two papers describing the develonment and demonstration of a new unfolding procedure involving pressure vessel surveillance dosimetry in PWRs. The complete methodology is contained in the LEPRICON code system and provides techniques for calculating pressure vessel fluences and then adjusting them, with reduced uncertainties, on the basis of surveillance dosimetry measurements and a benchmark data base. The present paper presents an application of these techniques to an existing on-line commercial reactor. Results indicate that the best estimate of the pressure vessel lifetime based on a limiting fluence above $1 \mathrm{MeV}$ of $2 \times$ $10^{19}$ neutrons $/ \mathrm{cm}^{2}$ is about $129 \pm 11$ effective full-power years, whereas the unadjusted estimate has an uncertainty twice as large.

- Reacarch sponsored by Electric Power Research Inssip ute.

Computing and Telecommunications Division.

Louisiana State University, Baton Rouge, LA.

Sacah Institute of Physicathe Hebrew University. Jerusalem, Israel.

\subsection{5}

\section{RECENT PROGRESS AND DEVELOPMENTS IN LWR-PV CALCULATIONAL METHODOLOGY*}

\section{R. E. Mrester $\quad$ B. L. Brondread $t$ M. L. Williant}

IAbstract of paper presented at the Fifth ASTM-Euratom Symposium on Reactor Dosimetry, Geesthacht, West Germany, September 24 28. 1984: Proc. Vol. I, p. 1639. Dordrecht, Netheriands (1985)]

New and improved techniques for calculating beltline surveillance activities and pressure vessel fluences with reduced uncertainties have recently been developed. These tochniques involve the combining of monitored in-core power data with diffusion theory calculated pin-by-pin data to yield absolute source distributions in $R-\theta$ and $R-Z$ geometries suitable for discrete ordinate transport calculations. Effects of finite core height, whenever necessary, can be considered by the use of a three-dimensional fluence rate synthesis procedure. The effects of a time-dependent spatial source distribution may be readily evaluated by applying the concept of the adjoint function and simplifying the procedure to such a degree that only one forward and one acjoint calculation are required to yield all the dosimeter activities for all beltline surveiliance locations at once. The addition of several more adjoint calculations using various group fluence rates as responses is all that is needed to determine all the pressure vessel group fluences for all beltline locations for any arbitrary source distribution.

- Research sponsored by the Ejectric Power Research Institute and U.S. Department of Energy.

'Computing and Telecommunications Division.

f Louisiana State University, Baton Rouge, LA.

\subsection{6}

\section{SENSITIVITIES OF THE FLUX SPECTRUM IN THE CAVITY OF A PWR TO VARIATIONS IN THE CORE SOURCE DISTRIBUTION*}

\section{B. L. Broadlead ${ }^{t}$ \\ R. L. Mherker}

[Abstract of Trons. Am. Nucl. Soc. 46, 659 (1984)]

As part of an on-going Electric Power Research Institute-sponsored project whose aim is the quantification and reduction of fluence uncertainties in the pressure vessel of operating pressurized water reactors (PWRs), calculations have been made of sensitivities necessary for the propagation of PWR core source distribution uncertainties to ti.: flux spectrum at locations of interest (e.g., the cavity or $T / 4$ pressure vessel locations) in the ANOI reactor. 
This study shows that due to the deep penetration of core source neutrons through the reactor internals, water gap and pressure vessel, only the top few source groups contribute appreciably to the detuctor responses in the pressure vessel and cavity locations. A procedure developed to take advantage of this property can yield sensitivities of all the detector group fluxes to the core source distribution in space to within 5\% with only five two-dimensional adjoint calculations.

- Recarch spoasored by US. DOE Ofiroe of Envizonmentes Recearch and Developmeat.

'Compating and Telecommunications Divisioe.

\subsection{7}

CORRELATIONS BETWEEN CALCULATED SURVEILLANCE DOSIMETER ACTTVTTIES AND PRESSURE VESSEL FLUXXS IN THE ARKANSAS NUCLEAR ONE-UNIT 1 REACTOR*

\section{R. E. Meerter $\quad$ B. L. Broadread}

[Abstract of Trans. Aen. Nucl. Soc. S9. 436 (1985)]

An illustration of the magnitudes of the cross-correlations between cavity dosimetry and fluxes at an important location in the presure vessel is given for the case of the ANO-1 reactor and provides a correspondence with previously reported flux covariance reduction factors. These correlations are seen to extend over energy regions far wider than would be inturitively expected because of the highly correlated nature of the calculated pressure vessel fluxes themselves, thus enhancing the range of pressure vessed flux informacion that is provided by the dosimetry. The high degree of correlation between the cavity dosimeters and pressure vesed nuxes shows that ex-vessel dosimetry can be successfully applied to reactor pressure vescel damage surveillance programs.

"Research sponsored by Electrix Power Rewarch Inatitute. 'Comperting and Telecommupications Divition.

\section{Reactor Physics Methods}

4.28

\section{ANALYSIS OF THERMAL REACTOR BENCHMARKS WITH DESIGN CODES BASED ON ENDF/B-V DATA*}

\author{
M. L. Williamst \\ R. Q. Wright \\ B. A. Worky \\ 0. Ozers \\ W. J. Eichs
}

[Abstract of Nuel. Tech. (in preas)]

Thermal reactor benchmark calculations have been performed with the "design codes" EPRICELL and PDQ using ENDF/B-V cross section data. The purpose of these calculations is to determine the quality of ENDF/B-V data for predicting reactor parameters when used with methods typically employed for power reactor analysis. This verification is essential if ENDF/B-V cross sections are to be used widely by the nuclear industry for reactor design, core reload, and core-follow studies.

The accuracy of the approximations in the design codes is demonstrated by comparison with rigorous bexchmark methods which use the same basic cross sections. It is shown that the deaign codes produce excellent results for several "numerical benchmarks," which were analyzed with Monte Carlo and discrete ordinates transport theory. After validating the methods approximations, the design codes are then used to calculate reactor parameters for a number of 
thermal systems. Many experimental configurations riot previously analyzed with ENDF/B-V data are included: $\mathbf{8}$ isolated rod resonance integral measurements, 21 different $\mathrm{UO}_{2}$ critical lattices and 6 mixed oxide critical lattices. Critical eigenvalues, and in some cases reaction rate ratios, are compared to experimental values for the benchmark lattices. In addition the timedependent isotopic composition of fuel pins in several power reactors is calculated using ENDF/B-V cross sections, and results are compared with experimental measurements.

It is found that ENDF/B-V, when used in typical reactor design codes, is an accurate data set for light water reactor analysis. Resonance integrals and reaction ratios for ${ }^{238} \mathrm{U}$ seem to be computed slightly high, but are within the uncertainty. The average $k_{\text {eff }}$ obtained for a wide variety of critical configurations is $1.002 \pm 0.002$. Critical $\mathrm{UO}_{2}$ eigenvalues are consistently overestimated slightly. The average eigenvalue obtained for the mixed oxide lattices is 1.0007 with a standard deviation of 0.0023 . Isotopic ratios generally show gcod agreement with measured values.

\footnotetext{
- Research sponsored by Electric Power Research Institute.

'Louisiana State University, Baton Rouge, LA.

FCoraputing and Telecommunication Division.

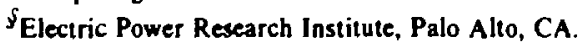

4.29

\section{IMPROVEMENTS IN CALCULATING NEITRON TRANSMISSION PROBABILITIES IN UNIT-CELL INTERFACE-CURRENT CODES*}

\author{
B. A. Worley
}

[Abstract of Nucl. Sci. Eng. 91. 293 (1985)]

A standard assumption used in unit-cell interface-current codes is that neutrons enter each spatial region with an isotropic angular distribution. This paper discusses the physical interpretation of this assumption and shows the magnitude oi the error introduced by it for a range of practical unit-cell geometries. An improvement upon the calculation of first-flight transmission probabilities for one-dimensional unit cells, based upon limiting the neutron source angular distribution to physically possible neutron flight directions, is then presented. For three-region problems, one additional calculation of a revised outer-region transmission probability is sufficient for determining all the revised transmission probabilities of interest. Calculation of the revised transmission probabilities requires only minor coding changes and eliminates the improper angular redistribution of neutrons at the region boundaries.

-Rescarch sponsored by U.S. DOE Office of Basic Energy Sciences.

\section{Performance Assessment of Waste Isolation Systems}

\subsection{0}

\section{RADIONUCLIDE MIGRATION PATHWAYS ANALYSIS FOR THE OAK RHDGE CENTRAL WASTE DISPOSAL FACILITY ON THE WEST CHESTNUT RIDGE SITE*}

\section{F. G. Pin J. P. Witherspoon ${ }^{\dagger}$ \\ D. W. Leet $\quad$ J. B. Cannont}

R. H. Ketelle ${ }^{t}$

(Abstract of ORNL/TM-9231, October 1984)

A dose-to-man pathways analysis is performed for disposal of low-level radioactive waste at the
Central Waste Disposal Facility on the West Chestnut Ridge Site. Both shallow land burial (trench) and aboveground (tumulus) disposal methods are considered. The waste volumes, characteristics, and radionuclide concentrations are those of waste streams anticipated from the Oak Ridge National Laboratory, the Y-12 Plant, and the C.دk Ridge Gaseous Diffusion Plant. The site capacity for the waste streams is determined on the basis of the pathways analysis.

The exposure pathways examined include (1) migration and transport of leachate from the waste disposal units to the Clinch River (via 
the groundwater medium for trench disposal and Ish Creek for tumulus disposal), and (2) those potentially associated with inadvertent intrusion following a 100-year period of institutional control: an individual resides on the site, inhales suspended particles of contaminated dust, ingests vegetables grown on the plot, consumes contaminated water from either an on-site well or from a nearby surface stream, and receives direct exposure from the contaminated soil.

It is fou id that either disposal method would provide effective containment and isolation for the anticipated waste inventory. However, the proposed trench disposal method would provide more effective containment than tumuli because of sorption of some radionuclides in the soil. Persons outside the site boundary would receive radiation doses well below regulatory limits if they were to ingest water from the Clinch River. An inadvertent intruder could receive doses that approach regulatory limits; however, the likelihood of such intrusions and subsequent exposure is remote.

* Research sponsored by U.S. Department of Energy.

${ }^{\dagger}$ Health and Safety Rese: $h$ Division.

Enerzy Division.

4.31

\section{APPLICATION OF PATHWAYS ANALYSES FOR SITE PERFORMANCE PREDICTION FOR THE GAS CENTRIFUGE ENRICHMENT PLANT AND OAK RIDGE CENTRAL WASTE DISPOSAL FACILITY*}

\author{
F. G. Pia $\quad$ E. M. Obion
}

(Abstract of paper presented at the Sixth Annual DOE. LLWMP Participants' Information Meeting, Denver, CO, September 11-13, 1984)

The suitability of the Gas Centrifuge Enrichment Plant and the Oak Ridge Central Waste Disposal Facility for shallow land burial of low. level radioactive waste is evaluated using pathways analyses. The analyses rely on conservative scenarios to describe the generation and migration of contamination and the potential human exposure to the waste. Conceptual and numerical models are developed using data from comprehensive laboratory and field investigations and are used to simulate the long-term transport of contamination to man. Conservatism is built into the analyses when assumptions concerning future events have to be made or when uncertainties concerning site or waste characteristics exist. Maximum potential doses to man are calculated and compared to the appropriate standards. The sites are found to provide adequate buffer to persons outside the DOE reservations. Conclusions concerning site sapacity and site acceptability are drawn. In reach: $=g$ these conclusions, some consideration is given to the uncertainties and conservatisms involved in the analyses. Analytical methods to quantitatively assess the sensitivity of the results to data uncertainty may prove useful in relaxing some of the conservatism built into the analyses. The applicability of such methods to pathways analyses is briefly discussed.

*Research sponsored by ONWI with the U.S. DOE Department of Nuclear Waste.

\section{PATHWAYS ANALYSES AND THEIR ROLE IN THE DECISION MAKING PROCESS FOR SELECTION OF LOW-LEVEL WASTE DISPOSAL SITES*}

\section{F. G. Pin $\quad$ E. M. Oblon}

(Abstract of paper presented at the Seventh Annual Symposium on Management of Uranium Mill Tailinga, Low-Level Waste and Hazardous Waste, Colorado State University, Fon Collins, CO, February $6-8,1985$ )

Pathways analyses have been extensively used to evaluate the suitability of proposed sites for disposal of low-level radioactive waste. The analyses rely on conservative scenarios to describe potential human exposure to the waste. Conceptual and numerical models are used to simulate the long-term transport of contamination to man, and additional conservatism generally is built into the analysis when assumptions concerning future events have to be made or when uncertainties concerning site or waste characteristics exist. This conservatism is useful in ascertaining 
whether the site provides an adequate buffer to persons outside the site boundary. In reaching conclusions concerning site capacity and site acceptability, however, considerations must be given to the uncertainties involved in the analysis. Analytical methods to quantitatively assess the sensitivity of the resul's to data uncertainties may piove uscful in the decision making process for site suitability.

- Kesearch sponsored by U.S. DOE Office of Nuclear Waste Fund.

4.33

\section{AUTOMATED SENSITTVITY ANALYSIS OF THE RADIONUCLIDE MIGRATION CODE UCR.NE-10.2*}

\author{
F. G. Pin \\ B. A. Worley \\ E. M. Otlow \\ R. Q. Wright ${ }^{\prime}$ \\ W. V. Harpert
}

(Abstract of paper to be presented at the International Topical Meeting on High-Level Waste Disposal Enginecring and Technotogy, Pasco, WA, September 24-26, 1985)

The Salt Repository Project (SRP) of the U.S. Department of Energy is performing ongoing performance assessment analyses for the eventual licensing of an undergiound high-level nuclear waste repository in salt. As part of these studies, sensitivity and uncertainty analyses play a major role in the identification of important parameters, and in the identification of specific data needs for site characterization. Oak Ridge National Laboratory has supported the SRP in this effort resulting in the development of an automated procedure for performing large-scale sensitivity analysis using computer calculus. GRESS, GRadient Enhanced Sof tware System, is a pre-compiler that can process FORTRAN computer codes and add derivative taking capabilities to the normal calculated results. The GRESS code is described and applied to the code UCBNE-10.2 which simulates the migration through an adsorptive medium of the radionuclide members of a decay chain. Conclusions are

drawn relative to the applicability of GRESS for more general large-scale modeling sensitivity studies, and the role of such techniques in the overall SRP sensitivity/uncertainty program is detailed.

- Recarch sponsored by U.S. DOE Oftice of Defense Waste and By-Products Management.

${ }^{t}$ Computing and Telocommunications Division.

FOfTre of Nuckear Waste Isolation, Columbus, $\mathrm{OH}$.

4.34

\section{GRESS - GRADIENT-ENHANCED \\ SOFTWARE SYSTEM - VERSION \\ D USER'S GUIDE*}

\section{E. M. OWlow}

(Abstract of ORNL/TM-9658, July 1985)

An automated procedure for performing sensitivity analyses has been developed. The procedure uses a new FORTRAN pre-compiler with computer calculus capabilities to generate the derivatives needed to set up sensitivity eruations. The new pre-compiler is called GRESS - Gradient Enhanced Software System.

GRESS enhances conventional FORTRAN programs with analytic differentiation of arithmetic statements. It handles any standard FORTRAN code which contains arithmetic text material as part of the source program. GRESS reads the FORTRAN source code text, redefines the variables and their storage locations, searches for arithmetic statements, translates the latter for gradient calculations, and then generates a new source program which now includes gradient capabilities in all arithmetic statements. GRESS, therefore, allows any standard FORTRAN sode to be upgraded to calculate any derivatives required, whether they be for internal use in a calculation (e.g. for iteration) or for external use (e.g. sensitivity studies).

-Research sponsored by U.S. DOE Office of Nuclear Waste Isolation. 


\section{Analysis of $\mathrm{CO}_{2}$ Impact on Climate}

4.35

\section{CARBON DIOXIDE BUILDUP AND CLIMATE: POTENTIAL IMPACTS*}

\author{
M. C. G. Hall
}

(Abstract of paper presented at the 1985 WATTec Conference, Knoxville, TN, February 12, 1985)

Many scientists are now concerned that a buildup of $\mathrm{CO}_{2}$ in the atmosphere could have a big impact on the earth's climate. The reason? When we us coal or oil to produce energy we also produce $\mathrm{CO}_{2}$. About half this $\mathrm{CO}_{2}$ lingers in the atmosphere, probably for centuries. While transparent to sunlight, this $\mathrm{CO}_{2}$ tends to trap the radiation coming up from the ground. The result is higher temperatures - the so-called greenhouse effect. Most estimates suggest that a doubling of $\mathrm{CO}_{2}$ in the atmosphere (expected some time next century) will raise average temperatures by about 1 to $4^{\circ} \mathrm{C}$. That may not sound like much of a threat, but the associated changes in regional climate would be. For example, a shift in seasonal weather patterns could wipe out the mid-West's grain harvest.

To plan effectively for its consequences, we need hard evidence that the $\mathrm{CO}_{2}$-induced climate change really will happen, and we need to know when and where it will hit hardest. Two sources of evidence are available to us: observations of climate change, and theoretical predictions of climate models. So far, the observational evidence is unconvinciag. Atmospheric $\mathrm{CO}_{2}$ has risen by $14 \%$ since 1880 , and temperatues have risen by $0.5^{\circ} \mathrm{C}$. This is about the expected greenhouse effect, but there's a snag. A warming of $0.5^{\circ} \mathrm{C}$ is easily attributable to the climate's natural variability. So at least for the time being we must rely on our other source of evidence - climate models.

The best climate models calculate preseures, winds, temperatures and humidities over the entire planet (stretching computers to their limits in the process). But do these models represent important physical processes well enough? It's hard to say until we understand the physical approximations that have been made and know how much uncertainty they introduce. This talk explains the physical reasoning behind climate models and describes the importance of recent steps to help understand and improve them.

-Research sponsored by U.S. DOE Carbon Diaxide Research Division of the OfTice of Basic Enerzy Sciences.

\subsection{6 \\ ESTIMATING THE RELIABILTY \\ OF CLIMATE MODEL PROJECTIONS - STEPS TOWARD A SOLUTION*}

\author{
M. C. G. Hall
}

(Abstract of chapter in Carbon Dioxide Stule-of-the-Art Report No. 3, The Polential Climatic Effects of Increasing Carbon Dioxide. M. C. MacCracken and F. M. Luther, Eds (in press)]

This article is part of the U.S. Department of Energy's State-of-the-Art report on the Potential Climatic Effects of Increasing Carbon Dioxide. It argues that the reliability of climate model projections is an issue of overriding importance in the $\mathrm{CO}_{2}$ problem. Estimating reliabili'y is difficult, though. There are serious shortconings to the conventional approaches of both modei intercomparison and validation against observation. The main conclusion is that the most promising (and perhaps the only) way to make meaningful steps towards a solution is to embark on a theoretical analysis of uncertainty, supported by a comprehensive sensitivity analysis.

The article starts by defining the terms used in uncertainty analysis and reviews existing methods of model validation in the context of $\mathrm{CO}_{2}$-climate modeling. Then methods of sensitivity analysis are reviewed. The adjoint method emerges as the only method currently available for general circulation models that is capable of giving the sensitivity information necessary for a comprehensive uncertainty analysis. Some preliminary results of this method are presented for the Oregon State University atmospheric general 
101 jit:

circulation model. The article concludes by recommending a systematic procedure to understand the effect of uncertainties in climate models.

"Research sponsored by U.S. DOE Carbon Diaxide Research Division.

4.37

\section{FEEDBACX IN THE CLMATE: ACCURACY OF FIRST-ORDER ESTIMATTS*}

\section{C. G. Hal}

[Abatract of toumal of the Atmospheric Sciences (in preasi)]

Climate feedbacks are extremely important in climate change. This note shows that the feedbacks in a climate model can be well understood in terms of first-order sensitivity theory. The first-order "sensitivity to feedback" is interpreted as the effect of one circuit around a feedback loop, and the total effect of feedback is accurately estimated by summing a geometrical progression. This method of estimating the effect of feedback is important because it can be applied with great efficiency to general circulation models, where the conventional approach of removing feedbacks and recalculating is prohibitively expensive.

-Research sponsorod by U.S. DOE Carbon Dioxide Research Division.
4.38

\section{EFFICIENT ESTMMATION OF FEEDBACX EFFECTS WITH APPLICATION TO CUMATIC MODELS*}

\author{
D. G. Cacaci M. C. G. Hall
}

[Abstract of J. Atmas. Sci. 41(13), 2063 (1984)]

This work presents an efficient method for calculating the sensitivity of a mathematical model's result to feedback. Feedback is defined in terms of an operator acting on the model's dependent variables. The sensitivity to feedback is defined as a functional derivative, and a method is presented to evaluate this derivative using adjoint functions. Typically, this method allows the individual effect of many different feedbacks to be estimated with a total additional computing time comparable to only one recalculation. The effects on a $\mathrm{CO}_{2}$-doubling experiment of actually incorporating surface albedo and water vapor feedbacks in a radiative-convective model are compared with sensitivities calculated using adjoint functions. These sensitivities predict the actual effects of feedback with at least the correct sign and order of magnitude. It is anticipated that this method of estimating the effect of feedback will be useful for more complex models where extensive recalculations for each of a variety of different feedbacks is impractical.

"Reacarch sponsored by U.S. DOE Division of Carbon Dioxide Research and OTtioe of Basic Energy Sciences. 
Section 5

RELIABILITY AND HUMAN FACTORS RESEARCH

$\frac{103}{104}$ 


\title{
5.0. INTRODUCTION
}

\author{
P. M. Haas
}

For the research program in the Reliability and Human Factors area, which was "born" in the turbulent "post-TMI era," the period of this report might best be described as a period of adolescence. There have been many indications of maturity and signs of great potential, along with occasional regression to early-development behavior. There has been visible growth (in both the qualitative and quantitative sense), some area; of developing stability, and definite trauma. Certainly most traumatic was the announcement in early CY 1985 by the USNRC Office of Nuclear Regulatory Research (the funding agency for virtually all of the human factors research) that they would no longer have a human factors research program after FY 1985. The program has survived this funding crisis and is emerging stronger, though smaller, with a much better developed sense of identity and more purposeful, thoughtful long-range planning.

The work reported here, most of it funded by the NRC, is grouped into four general areas: system reliability, human performance measurement, personnel qualifications and training, and program management. The program management activity is called out separately to highlight the fact that management of the ORNL-NRC program in this area was of itself a significant task, and, more importantly, that the program as a whole, over and above its individual technical projects, attained a measure of success and recognition in the field. Membership in and the presentation to the International Evaluation of Operational Practices (IEOP) Working Group (Abstract 5.28) is representative of the peer recognition of the program. The other three technical areas, while admittedly somewhat arbitrarily defined, are ones for which some considerable strength has developed and which suggest future areas of emphasis.

The system reliability area includes data for and analysis of reliability of overall systems or of system elements-hardware, software, humans, or facilities. (Development of a human reliability model of maintenance personnel is highlighted in a separate subtopic.) The central effort in this area, and by far the largest program, is the continued development and operation of the Centralized Reliability Data Organization (CREDO) for the DOE Liquid Metal Reactor (LMR) Program. A major accomplishment during this period for the program and, in fact, for the Laboratory and the DOE LMR program as well, was the initiation of a cooperative effort between DOE and Power Reactor and Nuclear Fuel Development Corporation (PNC) of Japan for joint operation of CREDO. In addition to the financial benefits to DOE and CREDO, the mutual exchange of data and cooperative development established in the agreement greatly enhance the programs of both countries and clearly establish CREDO as the leading international center for LMR reliability data.

The R\&D in human performance ineasurement ieported on here was all supported by NRC and was related to a program of simulator experiments initiated during the last reporting period. The selection of experimental issues was strongly driven by the short-term needs of the regulatory side of NRC (rather than the research side), and perhaps could have been improved with more thoughtful consideration of the underlying longer term research issues. However, the methodology 
developed for collecting and analyzing human performance data ising full-scope training simulators during routine training exercises represents a significant contribution to the industry. It involves automatic data collection from the simulator software, videotaping, observer checklists, and subjective ratings by operators involved and training supervisors focused on performance measures derived from task analysis. This approach to human performance is perhaps the most comprehensive used $w$ date in the nuclear industry and has the potential for practical applications in the industry as well as for research.

The research in the personnel qualifications and training area, also sponsored by NRC, focused on development of methodology and tools for use in evaluation of qualification and training programs/systems and in further research. Two automated tools (TSORT and TAPS) were produced which represent state-of-the-art developments. One in particular, TAPS, applies concepts from artificial intelligence (natural language) research to automaie the extraction of implicit job knowledge/skill requirements from descriptive task-analysis data. If fully developed, this tool could lead to complete automation of the "front-end" of the training system development process, a major problem for all training system developers.

As indicated above, the research program involving development of a computer model (MAPPS) to estimate the reliability of nuclear plant maintenance personnel is highlighted as a separate subtopic. This was a substantial effort involving evaluation of the model (including field testing) and technology transfer via a formal course, as well as model development The model development was reported in the last progress report. Efforts during this period included successful evaluation of the model and transfer to the user (NRC). This program will be complete with the final publication of the users' manual for the computer model. The model represents not only an excellent tool for use by the NRC in probabilistic risk assessment and other research and regulatory programs, but a significant contribution to the field of human reliability modeling. 


\section{System Reliability}

5.1

\section{CREDO: THE CENTRALIZED RELIABILITY DATA ORGANIZATION - A DATA BASE AND DATA ANALYSIS FOR ADVANCED REACTORS*}

\author{
H. E. Knee \\ P. M. Hans \\ S. L. Painter \\ M. D. Anderson
}

[Absernct of Nuclear Sofety 243), 300 (1985)]

The Centralized Reliability Data Organization (CREDO) is a data base and data analysis center that focuses on reliability, availability, and maintainability data from advanced reactors. Sponsored by the U.S. Department of Energy's (DOE's) Office of Breeder Technology Projects, CREDO is located at the Oak Ridge National Laboratory (ORNL).

The primary functions of CREDO are collection, screening, cataloging, storage, retrieval, analysis, and dissemination of reliability, availability, and maintainability data for advanced reactors. CREDO's two primary data sources are the Fast Flux Test Facility in Richland, WA, and the Experimental Breeder Reactor-II (EBR-II) in Idaho Falls, ID. In addition, CREDO collects Jata at a number of U.S. test facilities. Specifically, these are the test loops of the Energy Technology Engineering Center (ETEC), the General Electric Advanced Reactor System Department (GEARSD), the Hanford Engineering Development Laboratory (HEDL), and the Westinghouse Advanced Energy Systems Division (WAESD).

- Reacarch sponsored by U.S. DOE OfTice Breeder Technology Projects.

5.2

\section{HUMAN FACTORS REVIEW FOR NUCLEAR POWER PLANT SEVERE ACCIDENT SEQUENCE ANALYSIS*}

\author{
P. A. Krois $\quad$ P. M. Hans
}

(Abstract of paper to be presented at the 29th Human Factors Society Meeting. Baltimore, MD. September 29 - October 3. 1985)

This paper discusses work conducted to: (1) support the severe accident sequence analysis of a nuclear power plant transient based on an assessment of operator actions, and (2) develop a descriptive model of operator severe accident management. Operator actions during the transient are assessed using qualitative and quantitative methods. A function oriented accident management model provides a structure for developing techni al operator guidance on mitigating core damage and preventing radiological release.

- Research sponsored by U.S. Nuclear Regulatory Commission Ofice of Nuclear Regulatory Research. 
5.3

\section{HUMAN FACTORS REVIEW FOR SEVERE ACCIDENT SEQUENCE ANALYSIS (SASA)*}

\author{
P. A. Krois \\ P. M. Hans \\ J. J. Maming \\ C. R. Borel't
}

(Abstract of paper prosented at the Twelith Water Reactor Safety Information Meeting. National Burean of Sundards, Washington, DC, October 22-26, 1984)

The paper will discuss work being conducted during this buman factors review including: (1) support of the Severe Accident Sequence Analysis (SASA) Program based on an assessment of operator actions, and (2) development of a descriptive model of operator severe accident management. Research by SASA analysts on the Browns Ferry Unit One (BFI) anticipated transient without scram (ATWS) was supported through a concurrent assessment of operator performance to demonstrate contributions to SASA analyses from human factors data and methods. A descriptive model was developed called the Function Oriented Accident Management (FOAM) model, which serves as a structure for bridging human factors, operations, and engineering expertise and which is useful for identifying needs/deficiencies in the area of accident management. Research needs in accident management and potential uses of the FOAM model are described.

- Research sponsored by U.S. Nuclear Regulatory Commission Office of Nuclear Regulatory Research.

${ }^{\dagger}$ General Physica Corporation, Chattanooga, TN

5.4

\section{A REVIEW OF THE TREAT UPGRADE REACTOR SCRAM SYSTEM RELIABILITY ANALYSIS*}
D. F. Montagere
J. B. Fwaed ${ }^{t}$
P. A. Krois
T. C. Morelock ${ }^{t}$
H. E. Knee
J. J. Maming
P. M. Hans
K. W. Weat

(Abstract of ORNL/TM-9367, October 1984)

A triply redundant reactor scram systcm (RSS) has been designed for the transient reactor test facility (TREAT) reactor operated by Argonne National Laboratory. Because of the safety importance of the RSS, the Department of Energy contracted Oak Ridge National Laboratory to conduct an independent review of the RSS reliability analysis. The scope of the review included the human reliability analysis, failure modes and effects analysis, independent failures analysis, common cause failure analysis, and analysis of a dedicated microprocessor tester (DMT). Important findings from the review were that the component failure data were conservative, that inclusion of omitted failures would result in a larger estimated probability of an inadvertent trip, that the qualitative common cause failure analysis should consider contamination of air and hydraulic systems, and that the human reliability analysis underestimated the potential for human error. It was concluded that the reliability of the RSS is as high as can be reasonably achieved, and recommendations were made regarding the analytic approaches used to estimate the frequency of accidents at the TREAT facility and the human factors practices affecting use of the DMT.

- Research sponsored by U.S. DOE Office of Breader Technotogy Projects.

JBF Asrociater, Knoxville. TN.

Instrumentation and Controls Division.

\section{5}

\author{
CREDO DATA ANALYSIS: A \\ COMPARISON OF SODIUM AND \\ WATER VALVE RELIABILITIES*
}

\author{
S. L. Painter M. D. Andersou \\ H. E. Kee
}

(Abstract of paper to be presented at the International Conference on Nuclear Power Plant Aging, Availability Fac. tor and Reliability Analysis, San Diego, CA, July 8-12, 1985)

The Centralized Reliability Data Organization (CREDO) is a DOE-sponsored liquid metal reactor (LMR) component reliability data base and data analysis center located at Oak Ridge National Laboratory (ORNL). Since 1979, CREDO has been collecting data from experimental sodium reactors and various U.S. sodium 
test loops with the main emphasis being on those components which are unique to LMRs and which may have a significant impact on safety. Of the safety-related LMR components, valves are the most abundant, and CREDO has collected sufficient sodium valve operating data (over $\mathbf{2 0}$ million operating hours) to provide reliable estimates of sodium valve failure rates.
For this paper, sodium valves were characterized by size, seat design. actuator type, and seal type. Failure rates were calculated for each characterization using in-house statistical techniques. Finally, the failure rates obtained were compared to those of similar light-water reactor valves.

-Research sponsored by U.S. DOE OITice of Breader Technology Projects

\section{Human Performance Measurement}

\section{6}

THE EFFECTS OF SUPERVISOR
EXPERIENCE AND ASSISTANCE OF A
SHIFT TECHNICAL ADVISOR (STA)
ON CREW PERFORMANCE IN
CONTROL ROOM SIMULATORS

A. N. Beare ${ }^{t}$

D. L. Lassiter't

M. D. Doworan'

L. H. Gray

(Abstract of NUREG/CR-4280. ORNL/TM-9660, September [985]

This report describes the second experiment in a Nuclear Regulatory Commission-sponsored program of training simulator experiments to evaluate the effects of selected performance shaping factors on the performance of nuclear power plant control room operators. The factors investigated were the experience level of the Senior Reactor Operator (SRO) in the supervisor's role and the presence of a Shift Technical Advisor (STA) to ass.st the operating crew.

The experiment was conducted in the plantreferenced training simulator for a $1100-\mathrm{MWe}$ pressurized water reactor (PWR) plant. Data were collected from 20 inree-man crews of licensed operators. The crews were split into "high" and "low" experience groups on the basis of the supervisor's years of experience as an SRO. One half of the high- and low-SRO experience groups were assisted by an STA. The crews performed four exercises, a turbine tie-on and three plant casualties which ranged in severity from an uncomplicated steam generator iube rupture to a major steam line break with loss of coolant and primary makeup water.

\footnotetext{
- Recarch spossored by US. Nuclear Resulatory Commission Oftrce of Nuclear Regulatory Research.

'General Physics Corporation, Allants, GA.
}

\section{7}

\section{SIMULATOR EXPERIMENTS: EFTECTS OF EXPERIENCE OF SENIOR REACTOR OPERATORS AND OF PRESENCE OF A SHIFT TECHNICAL ADVISOR ON PERFORMANCE IN A BOILING WATER REACTOR CONTROL ROOM*}
A. N. Bearet
R. E. Dorris ${ }^{t}$

L. H. Gray

(Abstract of ORNL/TM-9327, October 1984)

This report describes the first experiment in a Nuclear Regulatory Commission-spor-ored program of training simulator experiments and field data collection to evaluate the effects of selected performance shaping factors on the performance of nuclear power flant control room operators. The factors investigated were the experience level of the Senior Reactor Operator (SRO) and the presence of a Shift Technical Advisor (STA).

Data were collected from 16 two-man crews of licensed operators (one SRO and one RO). The crews were split into "high" and "low" SROexperience groups on the basis of the years of experience of the SROs as SROs. One half (4 of the 8 crews in each group) of the high- and lowSRO experience groups were assisted by an STA 
or an SRO acting as an STA. The crews responded to four simulated plant casualties which ranged in se.erity fro:n an unoomplicated turbine trip to an anticipated transient without scram (ATWS).

Two sets of content-referenced performance measures were derived from task analyses of the procedurally correct response; to the four casualties. One set of measures was focused on task performance, e.8., counts $0^{*}$ : subtasks initiated, task elements performed correctly, and the time taken to execute the required sequences. The second set of measures was based on control of system parameters, i.e., time-out-of-band and root-mean-square (RMS) etror for reactor water level and pressure. System parameters and control manipulations were recorded by the computer controlling the simulator. Data on communications and procedure use were obtained from checklists completed during review of videotapes of the exercises. Questionnaires were used to collect subject biographical information and data on the participants' subjective workload during each simulated casualty.

No significant differences in overall performance were found between groups led by high" (25-114 months licensed as an SRO) and Tow" ( $1-17$ months as an SRC) experience SROs. However, crews led by "low" experience SROs tended to have shorter tasc performance times than crews led by 'high' experience SROs. Although a tendency for the STA-assisted groups to score higher on four of the five measures was observed, the presence of the STA had no statistically significant effect on overall team performance. The correlation between individual performance, as measured by four of the task performance measures, and experience, measured by months as a licensed opera.tor, was not statistically significant, nor was the correlation between task performance and recency of simulator training.

There were no differences in the perceived workloads of SROs assisted by STAs and those who were not. The reported workload of STAs was significantly lower than the workload of ROs and SROs for all sequences. There were significant differences in perceived workload for the four operating sequences. ATWS was highest, followed by Safety/Relief Valve Fails Open, followed by Loss of Feedwater and Turbine Trip. which did not differ significantly.

The results of the experiment suggested that, within the range of SRO experience examined, years of control room experience may not be an important predictor of performance.

- Research spansored by U.S. Nectear Regubtory Canmisuiga Oftro of Nuclear Regulatory Research.

'General Physios Corporation, Allata, GA.

58

\section{SMULATOR EXPERMIENTS: EPTCTS OF NPP OPERATOR EXPDIIZNCE ON PERPONMNCZ*}

\section{A. N. Beare $\quad$ L. H. Graj}

iAbstract of paper preated at the Treffl Wetar Reactor Safety Information Meetieg. Nationsl Buran of Standarts, Washiagloa, DC, October 22-26, 1944; Proc NUREG/CP-Coss, Vol. 6, p. 17 (1984)]

Experiments are being conducted on Nuclear Power Plant (NPP) Control Room training simulators by the Oak Ridge National Laboratory, its subcontractor General Physics Corporation, and participating utilities. The experiments are sponsored by the Nuclear Regulatory Commission's (NRC) Human Factors and Safeguards Branch, Division of Risk Analysis and OFerations, and are a continuation of prior research using simulators, supported by field data collection, to provide a technical basis for NRC human factors regulatory issues concerned with the operational safety of nuclear power plants.

During the FY83 research, a simulator experiment was conducted at the control room simulator for a GE Boiling Water Reactor (BWR) NPP. The research subjects were licenwed operators undergoing requalification training and shift technical advisors (STAs). This experiment was designed to investigate the effects of (a) senior reactor operator (SRO) experience, (b) operating crew augmentation with an STA, and (c) practice, as a crew, upon crew and individual operator performance, in response to anticipated plant transients. 
Sixtoen two-man crews of licensed operators were employed in a $2 \times 2$ factorial design. The SRDs leading the crews were split into high" and Tow" experience groups on the basis of their years of experience as an SRO. One half of the high- and low-SRO experience groups were assisted by an STA. The crews responded to four simulated plant casualties. A five-variable set of content-referenced performance measures was derived from task analyses of the procedurally correct responses to the four casualties. System parametes and control manipulations were recordeal oy the computer controlling the simulator. Data on communications and procedure use were obtained from analysis of videotapes of the exercises. Questionnaires were used to collect subject biographical information and data on subjective workload during each simulated casualty.

For four of the five performance measures, no significant differences were found between groups led by "high" (25-114 months) and 7ow' (1-17 months) experience SROs. However, crews led by "low" experience SROs tended to have significantly shorter task performance times than crews led by high" experience SROs. The presence of the STA had no significant effect on overall team performance in responding to the four simulated casualties.

The FY84 experiments are a partial replication and extension of the FY83 experiment, but with PWR operators and simulator. Twentyseven crews (24 three-man crews of licensed operators and three groups of pre-license trainees, plus STAs augmenting 12 of the crews) will respond to five operating sequences representing a range of difficulty from routine to very severe accidents. Results of the FY84 experiments will be available by March 1985.

- Research sponsored by U.S. Nuclear Regulatory Commiscion Ortice of Nuclear Reculatory Research.

'General Physica Corporation. Chattanooza. TN.
5.9

\section{CONTROL ROOM OPERATOR PERFORMANCE RESEARCH*}

\author{
L. H. Gray \\ P. M. Hans \\ J. J. Manine J. C. Lowry ${ }^{\dagger}$
}

[Abstrect of Trause Ane Nuct. Soc. 4h, 309 (1984)]

Oak Ridge National Laboratory, under the sponsorship of the U.S. Nuclear Regulatory Commission (NRC) Office of Nuclear Regulatory Research, has for the past several years been conducting control room operator performance research using commercial nuclear power plant (NPP) training simulators. The effort has been performed under two programs: the SafetyRelated Operator Actions (SROA) program, and the Training Simulator Experiments (TSE) program.

The SROA program's primary effort involved collection and assessment of data from simulator training exercises and from historical records of abnormal/emergency events that occurred in operating plants (field data). These data can be used to develop criteria for acceptability of the use of manual operator action for safety-related functions. The program also included studies of training simulator capabilities, of procedures and data for specifying and verifying simulator performance, and of methods and applications of task analysis.

In 1983 the SROA program was completed, with the development of an improved process for field data collection and of a proposed structure for design evaluation using a systems model. A simplified and preliminary systems model was developed and tested.

The TSE program, initiated in 1983, has examined effects on performance caused by variations of a basic crew consisting of a reactor 
operator (RO) and a senior reactor operator (SRO), sometimes augmented by a shift technical advisor (STA).

The methodologies and procedures developed under the SROA program were used in a series of controlled experiments conducted at an operating BWR plant's training simulator. Plant operating crews' performances during four standardized operating sequences were recorded and analyzed. None of the variables of education, training, and experience showed signuficant correlation with overall performance.

- Rescarch sponocred by U.S. Nuclear Regalatory Commincioa Office of Nectar Regulatory Rexearch.

'Nuclear Regulatory Commixcion, Washington, DC.

5.10

\section{NUCLEAR POWER PLANT CONTROL ROOM OPERATORS PERFORMANCE RESEARCH*}

\section{H. Gray P. M. Hase}

(Abarant of paper presented at the Human Factors Society 28th Annual Meeting on New Frontiers for Science and Technology. Sir Antonio, TX, October 22-26, (984)

A research program is being conducted by the Oak Ridge National Laboratory for the U.S. Nuclear Regulatory Commission to provide information on the performance of nuclear power plant control room operators when responding to abnormal/emergency events in the plants and in full-scope training simulators. The initial impetus for this program was the need for data to assess proposed design criteria for the choice of manual versus automatic action for accomplishing safetyrelated functions during design basis accidents. The program also included studies of training simulator capabilities, of procedures and data for specifying and verifying simulator performance, and of methods and applications of task analysis.

-Research sponsored by U.S. Neclear Regulatory Comminsion Otice of Nuclear Regulatory Research.

\subsection{1}

\section{COLISCTION AND ANALYSISL TRAIING SIMULATOR DATA*

\author{
P. A. Krois $\quad$ P. M. Hans
}

(Abstract of paper preaented at the 1985 IEEE Third Coaference on Humas Fuctors and Power Plants, Mantercy. CA June '3-27, 1985)

The purposes of this paper are: (1) to review the objectives, approach, and results of a series of research experiments performed on nuclear power plant training simulators in support of regulatory and research programs of the U.S. Nuclear Regulatory Commission (NRC), and (2) to identify general research issues that may lead to an improved research methodology using the training simulator as a field setting. Research products consist of a refined field research methodology, a data store on operator performance and 'specific results pertinent to NRC regulatory positions. Issues and potential arvances in operator performance measurement are discussed.

- Research sponsored by U.S. Nuclear Requlatory Commir sion Office of Nuclear Regulatory Revearch. 


\section{Personnel Qualifications and Training}

5.12

\section{A CLASSROOM TRAINING REVISION METHODOLOGY *}

\section{R. J. Carter}

(Abstract of paper presented at the Human Factors Society 28th Annual Meeting on New Frontiers for Science and Technology, San Antonio. TX, October 22-26, 1984)

A methodology was described to revise program deficiencies in Army classroom training identified during training effectiveness evaluations. The baseline for its design approach was discussed. The revision methodology's content and structure were presented in detail.

-Research sponsored by U.S. Army Research Institute for the Behavioral and Social Sciences, Fort Bliss, Field Unit, El Paso, TX.

\subsection{3}

\section{NUCLEAR POWER PLANT TRAINING SIMULATOR FIDELITY ASSESSMENT*}

\section{R. J. Carter K. R. Laughery ${ }^{-}$}

(Abstract of paper to be presented at the 29th Fiuman Factors Society Mecting, Baltimore. MD, September 29 -. October 3 , 1985)

The fidelity assessment portion of a methodology for evaluating nuclear power plant simulation facilities in regard to their appropriateness for conducting the Nuclear Regulatory Commission's operating test was described. The need for fidelity assessment, data sources, and fidelity data to be collected is addressed. Fidelity data recording, collection, and analysis is discussed. The processes for drawing conclusions from the fidel- ity assessment and evaluating the adequacy of the simulator control-room layout were presented.

- Research sponsored by J.S. Nuclear Regulatory Commis-
sion Office of Nuclear Regulatory Research.
+Micro Analysis and Design, Boulder, CO.

5.14

\section{A METHODOLOGY FOR THE EVALUATION OF NUCLEAR POWER PLANT SIMULATION FACLLTIES*}

\section{R. J. Carter K. R. Laughery ${ }^{\dagger}$}

(Abstract of paper presented at the 1985 Eastern Simulation Conference, Norfolk, VA, March 3-8, 1985)

A methodology designed to evaluate the acceptability of nuclear power plant simulation facilities for use in the simulator-based portion of the Nuclear Regulatory Commission's operator licensing examination is described. It is to be utilized during two phases of the life-cycle, initial simulator evaluation and recurrent evaluation. Initial evaluation is to be performed either when a simulator is acquired or as soon as is practical for existing simulators, and is aimed at ensuring that the simulator provides an accurate representation of the reference plant. There are two components of initial evaluation: fidelity assessment and a direct determination of the simulator's adequacy for operator testing. Recurrent evaluation is aimed at ensuring that the simulator continues to accurately represent the reference plant throughout the life of the simulator. It involves three components: monitoring reference plant changes, monitoring the simulator's hardware, and examining the data from actual plant transients as they occur.

- Rescarch sponsored by U.S. Nuclear Regulatory Commission Office of Nuclear Regulatory Research.

'Micro Analysis and Design, Boulder, CO. 
5.15

\section{TRAINING RELATED RESEARCH AND DEVELOPMENT CONDUCTED AT OAK RIDGE NATIONAL LABORATORY FOR THE U.S. NUCLEAR REGULATORY COMMISSION*}

\section{P. M. Haas}

(Abstract of paper presented at the 1985 IEEE Third Conference on Human Factors and Power Plants, Monterey, CA, June 23-27, 1985)

For a number of years Oak Ridge National Laboratory (ORNL) has conducted a sizeable program of human factors research and development in support of the Office of Nuclear Regulatory R-search of the U.S. Nuclear Regulatory Commission (NRC). The history of this effort has $\mathrm{i}:$ : many ways paralleled the growth of human factors R\&D throughout the nuclear industry and the program has contributed to advances in the industry as well as to NRC regulatory and research programs. This paper reviews the majo: projects and products of the program relevant ts training and concludes with an identification of future R \&D needs.

- Research sponsored by U.S. Nuclear Regulatory Commission Office of Nuclear Regulatory Research.

\subsection{6}

\section{NUCLEAR POWER PLANT SIMULATION FACILITY EVALUATION METHODOLOGY*}

\section{P. M. Haas $\quad$ R. J. Carter K. R. Laughery ${ }^{\dagger}$}

(Abatract of paper presented at the 1985 IEEE Third Conference on Human Factors and Power Plants, Monterey, CA, June 23-27, 1985)

A methodology for evaluation of nuclear power plant simulation facilities with regard to their acceptability for use in the U.S. Nuclear Regulatory Commission (NRC) operator licensing exam is described. The evaluation is based primarily on simulator fidelity, hut incorporates some aspects of direct operator/trainee performance measurcment. The panel presentation and paper discuss data requirements, data collection, data analysis and criteria for conclusions regarding the fidelity evaluation, and summarize the proposed use of direct performance measurement. While field testing and refinemer. $l$ of the methodology are recommended, this initial effort provides a firm basis for NRC to fully develop the necessary methodology.

-Research sponsored by U.S. Nuclear Regulatory Commission Office of Nuctear Regulatory Research.

'Micro Analysis and Design, Boulder, $C O$.

\subsection{7}

\section{METHODS FOR EVALUATTON OF NUCLEAR POWER PLANT PERSONNEL TRAINING AND ENTRY LEVEL QUALIFICATIONS*}
P. M. Hans
C. C. Jorgeasea
D. L. Selby
J. C. Lowry ${ }^{\prime}$

[Abstract of Trans. Am. Nucl. Soc. 46, 307 (1984)]

The U.S. nuclear power industry, led to a great extent by the Institute of Nuclear Power Operations and the U.S. Nuclear Regulatory Commission (NRC), has extensive efforts under way to assess, improve, and systematize the process of training of nuclear power plant (NPP) personnel. This paper summarizes early results and the current status of an NRC-funded research program at the Oak Ridge National Laboratory (ORNL) to provide the methods and technical basis for NRC to implement the systems approach to training programs and entry level qualifications of NPP control room personnel.

The first year's effort (March 1982 to August 1983) focused on an adaptation of the Systems Approach to Training (SAT) process to NRC needs. The existing literature on instructional systems development and SAT was reexamined with special emphasis on evaluation of training program effectiveness rather than design and development. A high-level "model" of the SAT for use in the nuclear industry was outlined to 
serve as a framework for NRC evaluation. Twelve checklists were proposed with rating scales for NRC to use to evaluate each of the major elements of the SAT process outlined in the model. Field testing by end users for practicality, acceptability, or utility prior to full implementation was strongly recommended.

A technique for ranking possible plant malfunctions as to their importance to operator training was developed. The ranking scheme is compatible with a fully implemented SAT-based training system, but is designed with subjective scales so that it can be used in the interim before complete and comprehensive data exist. Potential plant malfunctions are rated along three dimensions: consequence, frequency of occurrence, and difficulty of operator response.

Two other resuits produced during the course of development of the model and checklists were (a) preliminary identification of key variables (performance shaping fastors) to be considered in NPP control room personnel qualifications and training and (b) an illustrative media selection model that might be used in the development of an instructional delivery system for NPP control room operators.

The current phase of the research (August 1983 to September 1984) is extending the initial work to adapt, develop, and field test methods for NRC to evaluate training and entry level qualifications.

An automated methodology for selection of tasks into various training categories has been developed. The program, currently on an IBM personal computer, is fully interactive for both data entry and analysis and generates recommended allocation categories for each task, rankordered preferences for task allocation between categories, and rank-ordered preferences for tasks within categories.

A new method for identification of entry level skill aptitudes and knowledge requirements has been developed. It links a behavioral or in-plant task description to a taxonomic or psychological task description. This linkage permits the rapid identification of both personal aptitude requirements and potential for measurement tests.
As the result of efforts by both industry and NRC to assess and improve operator training and qualifications, the U.S. nuclear industry is moving to adaptation of the SAT, and the NRC is in parallel moving to adapt SAT methodology in their evaluation of training and qualifications. The research program summarized in this paper is intended to provide the methodology and technical basis for NRC's evaluation. A framework for an SAT-based evaluation process has been developed and current efforts will develop the specific methods and tools to implement the process.

- Research sponsored by U.S. Nuclear Regulatory Commission Office of Nuclear Regulatory Research.

'Nuclear Regulatory Commission, Washington, DC.

5.18

\section{NUCLEAR POWER PLANT PERSONNEL QUALIFICATIONS AND TRAINING: TSORT - AN AUTOMATED TECHNIQUE TO ASSIGN TASKS TO TRAINING STRATEGIES*}

\section{C. Jorgensea}

[Abstract of ORNL/TM-9308/VI (NUREG/CR-3481, Vol. 1), October 1984]

This report discusses TSORT, a technique to assist the Nuclear Regulatory Commission (NRC) in two areas: the first is to provide a standardized method to select tasks for use in NRC-sponsored training research and the second is to evaluate whether training program developers have allocated nuclear power plant tasks to appropriate training strategies. The TSORT structure is presented, including training categories selected, dimensions of task information considered, measurement metrics used, and a guide to application. TSORT is implemented as an automated software tool for an IBM-PC. It uses full-color graphics and interactive menu selection to provide NRC with a variety of evaluation options including: rank ordering of training strategies reasonable for each task, rank ordering of tasks within strategies, and a variety 
of special analyses. The program code is also piesented along with a comprehensive example of 20 realistic tasks illustrating each of 17 options available.

- Research sponsored by U.S. Nuclear Regulatory Comminsion Office of Nuctear Regulatory Rewearch.

\subsection{9}

\section{INITAL DEVELOPMENT OF AN AUTOMATED TASX ANALYSIS PROFILING SYSTEM (TAPS)*}

\section{C. Jargenea}

(Abstract of paper presented at the Human Factors Society 28th Annual Meeting on New Frontiers for Science and Tectbology, San Aptonio, TX, Octobet 22-26, 1984)

A program for automated task analysis is described. Called TAPS (task analysis profiling system), the program accepts normal English prose and outputs skills, knowledges, attitudes, and abilities (SKAAs) along with specific guidance and recommended ability measurement tests for nuclear power plant operators. A new method for defining SKAAs is presented along with a sample program output.

* Research sponsored by U.S. Nuctear Regulatory Commission OfTice of Nuclear Regulatory Research.

5.20

\section{NUCLEAR POWER PLANT PERSONNEL QUALIFICATIONS AND TRANING: TAPS - THE TASK ANALYSIS PROFILING SYSTEM*}

\section{C. Jorgenem}

[Abatract of ORNL/TM-9308/V2 (NUREG/CR-3481, Vol. 2), June [985]

This report discusses an automated task analysis profiling system (TAPS) designed to provide a linking tool between the behaviors of nuclear fower plant operators in performing their tasks and the measurement tools nea sary to evaluate their in-plant performance. TAPS assists in the identification of the entry-level still, knowledge, ability and attitude (SKAA) requirements for the various tasks and rapidly associates them with measurement tests and human factors principles. This report describes the development of TAPS and presents its first demonstration. It begins with characteristics of skilled human performance and proceeds to postulate a cognitive model to formally describe these characteristics. A method is derived for linking SKAA characteristics to measurement tests. The entire process is then automated in the form of a task analysis computer program. The development of the program is detailed and a user guide with annotated code listings and supporting test information is provided.

- Research sponsored by U.S. Noctear Regulatory Comminion Orfice of Nuclear Regulatory Research.

5.21

\section{DEVELOPMENT OF METHODS FOR NUCLEAR POWER PLANT PERSONNEL QUALIFICATIONS AND TRAINING*}
C. C. Jorgensea
R. J. Carter

[Abatract of paper presented at the Twelfth Water Reactor Safety Information Mecting. National Bureau of Standside, Washington, DC, October 22-26, 1984; Proc. NUREG/CP-0058, Vol. 6, p. 28 (1984)]

The Nuclear Regulatory Commission (NRC) has proposed that additions and revisions should be made to Title 10 of the Code of Federal Regulations, Parts 50 and 55, and to Regulatory Guides 1.8 and 1.149. Oak Ridge National Laboratory (ORNL) is developing methods and some aspects of the technical basis for the implementation and assessment of training programs, personnel qualifications, and simulation facilities to be designed in accordance with the proposed rule changes. The paper describes the three methodologies which were developed during the FY-1984 research. The three methodologies are: (1) a task sort procedure (TSORT); (2) a simulation facility evaluation methodology: and (3) a task analysis profiling system (TAPS). 
The task sort procedure was developed to determine the training strategy which should be applied to a given task. It acomplishes this by sorting tasks into nine categories, each of which is defined along ten dimensions. TSORT provides rank-ordered preferences for task allocation between and within categories and has the ability to estimate a dollar loss incurred through failure to train on a task. The simulation facility evaluation methodology is being developed to certify simulation facilities for use in the simulator-based portion of the licensing examination. It is to be utilized during two phases of the life-cycle, initial simulator testing and recurrent evaluation. The initial testing phase is aimed at ensuring that the simulator provides an accurate representation of the reference plant, while recurrent evaluation is aimed at ensuring that the simulator continues to accurately represent the reference plant throughout the life of the simulator. The task analysis profiling system has been designed to support training research. It draws on artificial intelligence concepts of pattern matching to provide an automated task analysis of normal English descriptions of job hehaviors. TAPS development consisted of creating a precise method for the definition of skills, knowledge, abilities, and attitudes (SKAA), and generating SKAA taxonomic elements. It systematically outputs skills, knowledge, attitudes, and abilities, and information associated with them.

-Research sponsored by U.S. Nuclear Regul' tury Cornmission Office of Nuctear Regulatory Research.

5.22

\section{A SELECT REVIEW OF THE RECENT (1979-1983) BEHAVIORAL RESEARCH LTTERATURE ON TRAINING SIMULATORS*}

\author{
K. R. Laxghery ${ }^{\dagger}$
}

[Abstract of ORNL/TM-9445 (NUREG/CR-4206), May [985]

This report summarizes some selected reports of behavioral research performed in the years 1979-1983 on training simulator application technology and discusses findings related to nuclear power plant operators' simulator training. Findings are organized as related to the design, testing, and use of training simulators. Topics include Simulator Fidelity in Training Effectiveness, Operator Performance Measurement, Measuring Simulator Effectiveness, and Simulator Utilization Practices.

- Research sponsored by U.S. Nuclear Regulatory Commission Office of Nuclear Regulatory Reacarcb.

'Micto Analysis and Design, Boulder, $\mathrm{CO}$.

\section{Human Reliability Assessment}

\subsection{3}

\section{THE MAINTENANCE PERSONNEL PERFORMANCE SIMULATION (MAPPS) MODEL: A HUMAN RELIABILITY ANALYSIS TOOL*}

\section{H. E. Knee}

(Abstract of paper to be presented at the International Conference on Nuclear Power Plant Aging. Availability Factor and Reliability Analyzis, San Diego, CA, July 8-12, 1985)

The development of the Maintenance Personnel Performance Simulation (MAPPS) Model has been sponsored by the U.S. Nuclear Regulatory
Commission (NRC) and was accomplished by Oak Ridge National Laboratory (ORNL) in conjunction with Applied Psychological Services, Inc. (APS).

MAPPS is a task-oriented simulation model that addresses person-machine, person-environment, and person-person relationships in the accomplishment of maintenance activities. It accounts for the interdependencies between various subelements of a maintenance task and includes a number of important performance shaping factore.

Although the model has been specifically developed to provide maintainer reliability data 
for input into probabilistic risk assessment (PRA) studies, it also has the ability to provide input for analyzing, designing, and optimizing the maintenance structure.

In order to ensure that MAPPS meets the goals for which it was designed, it was subjected to a comprehensive evaluation. These efforts, w lich have just recently been completed, addressed a number of issues related to its validity, practicality, acceptability, and usefulness and utilized data obtained from direct observation of maintenance in the field, expert consensus, and a case study approach. One of the validity issues addressed was the ability of MAPPS to predict overall task performance time. Field data collection for a source range channel calibration task and a reactor pressurizer wide-range level calibration task resulted in observed times of $\mathbf{7 3 . 6}$ minutes and 119.8 minutes, respectively. MAPPS' estimates for these tasks were $\mathbf{7 8 . 0}$ minutes and 117.0 minutes, respectively. The overall evaluation efforts led to positive confirmation on all issues addressed.

The purpose of this paper is to provide a description of the model and the results of the evaluation efforts. It will also present potential applications of the MAPPS model.

- Research sponsored by U.S. Nuclear Regulatory Commission Orfice of Nuclear Regulatory Research.

5.24

\section{MAINTENANCE PERSONNEL PERFORMANCE SIMULATION (NAPPS) MODEL: OVERVIEW AND EVALUATION EFFORTS*}

\author{
H. E. Knee P. M. Hans \\ T. G. Ryan' A. I. Sieget \\ W. D. Barttert J. J. Wout
}

[Abstract of paper presented at the Twelrth Water Reactor Safety Information Meeting. National Bureau of Standards, Washington, DC, Octobet 22-26, 1984; Proc. NUREG/CP.0058, Vol. 6. p. 61 (1984)]

The development of the MAPPS model has been completed and the model is currently undergoing evaluation. These efforts are addressing a number of identified issues concerning practicality, acceptability, usefulness, and validity. Preliminary analysis of the eve'uation data that has been collected indicates that MAPPS will provide comprehensive and reliable data for PRA purposes and for a number of other applications.

The MAPPS computer simulation model provides the user with a sophisticated tool for gaining insights into tasks performed by NPP mainte.jance personnel. Its wide variety of input parameters and output data makes it extremely flexible for application to a number of diverse applications. With the demonstration of favorable model evaluation results, the MAPPS model will represent a valuable source of NPP maintainer reliability data and provide PRA studies with a source of data on maintainers that has previously not existed.

- Research spoasored by U.S. Nuclear Regulatory Commiesion OrTice of Nuclear Regulatory Research.

${ }^{\prime}$ Nuclear Regulatory Commission, Washington, Dr

FApplied Psychological Services, Inc., Waype, PA.

\subsection{5}

\section{MAINTENANCE PERSONNEL PERFORMANCE SIMULATION (MAPPS) MODEL: DESCRIPTION OF MODEJ . CONTENT, STRUCTURE, AND SENSITTVITY TESTING•}
A. I. Sieget ${ }^{t}$
W. D. Bartter ${ }^{t}$
J. J. Woif
H. E. Knee

(Abstract of NUREG/CR-3626, Vod. 2 (ORNL/TM-9041/V2), December [984]

The MAintenance Personnel Performance Simulation (MAPPS) model is a computer representation of important aspects of behavior with regard to the performance reliability of nuclear power plant maintenance personnel (referred to throughout this report as "maintainers"). The primary purpose of the model is to provide information conserning maintenance effectiveness for input into probabilistic risk assessment studies. The model's generality, coupled with its generous selection of input parameters, allows it to be applied in a number of other 
contexts requiring maintainer performance information. A summary description of the functional characteristics of the MAPPS model, along with a brief review of its development and anticipated uses, was presented in Volume 1 of this series. This volume (Volume 2) presents supporting technical material. It specifies major aspects of the model, including structural detail, limitations, assumptions, programming structure, and the rationale for the model's contents. Since different readers may wish to concentrate on different sections, the report is written so that a degree of selective reading will be possible. However, the document $i$ organized as a unified entity, and the vario'is sections complement and supplement each other.

- Research sponsored by US. Nuclear Regulatory Commission Office of Nuclear Regulatory Research.

${ }^{\dagger}$ Applied Psychological Services, Inc., Wayne, PA.

\subsection{6}

\section{THE MAINTENANCE PERSONNEL PERFORMANCE SIMULATION (MAPPS) MODEL*}

\section{A. I. Siegel $t^{\dagger}$ W. D. Bartter ${ }^{\dagger}$ J. J. Woirt H. E. Knee \\ P. M. Hans}

(Abstract of Trans. Am. Nucl. Soc. 46, 298 (1984); also paper presented at the Human Factors Society 28th Annual Meeting on New Frontiers for Science and Technology, San Antonio. TX, October 22-26, 1984]

The maintenance personnel performance simulation (MAIPS) model was developed to provide a tool for developing required insights relative to nuclear power plant inaintenance to provide maintenance-oriented human performance reliability data for probabilistic risk assessment purposes.

MAPPS is a task-oriented, computer-based model for simulating nuclear power plant maintenance activities. It includes environmental, motivational, task, and organizational variables which influence personnel performance reliability and yields information such as predicted errors, personnel requirements, areas of maintainer stress and fatigue, performance time, and required maintainer ability levels for any corrective or preventive maintenance action in nuclear power plants.

The model sequentially simulates the performance of each subtask involved in total task completion. Within the logic, a number of important concepts are included such as various types of stress, environmental effects, quality of work situations, and inherent maintainer characteristics. These interact within the MAPPS model in accordance with the flow logic which includes stochastic features to account for intra- and interindividual situational and contextual differences.

The MAPPS model generates a large spectrum of output data that can be utilized by an analyst to gain a detailed insight into the task being simulated. These data can be generated on four levels: task leve!, subtask level, work shift level, and iteration level.

The mode! was subjected to sensitivity testing wherein a number of computer simulations were run to evaluate the reasonableness of its output and the output trends when the input daca are systematically varied.

The model's generality, flexibility, and richness of input and output parameters makes it useful for a number of different types of applications.

- Research sponsored by U.S. Nuclear Regulatory Commission Orfice of Nuclear Regulatory Research.

${ }^{\prime}$ Applied Psychological Services, Inc., Wayne, PA.

\subsection{7}

\section{MAINTENANCE PERSONNEL PERFORMANCE SIMULATION (MAPPS) MODEL: SUMMARY DESCRIPTION*}

$$
\begin{aligned}
& \text { A. I. Sieget }{ }^{t} \text { W. D. Bartter }{ }^{t} \\
& \text { J. J. Wolf H. E. Knee } \\
& \text { P. M. Hass }
\end{aligned}
$$

[Abstract of ORNL/TM-904]/VI (NUREG/CR-3626, Vol. 1), May 1984]

A summary description is presented of the rationale for and the content and structure of the 
MAintenance Personnel Performance Simulation (MAPPS) model. The MAPPS model is a generalized stochastic computer simulation model developed to simulate the performance of maintenance personnel in nuclear power plants. The MAPPS model considers work place, maintenance technician, motivation, human factors, and task-oriented variables to yield predictive information about the effects of these variables on successful maintenance task performance. MAPPS provides human performance reliability information pertinent to probabilistic risk asse sment, regulatory decisions, and maintenance personnel requirements. The model, which is drawn from a firm research analytic base, was examined for disqualifying defects from a number of viewpoints and its sensitivity was extensively tested. The MAPPS model is believed to be ready for initial and controlled applications which are in conformity with its purposes.

\footnotetext{
- Research sponsorad by U.S. Nuclear Regulatory Commiscion Otice of Nuclear Regulatory Reseanth.

'Applied Psychological Services, Inc, Wayne, PA.
}

\section{Human Factors Program Management}

\subsection{8}

\section{HUMAN FACTORS RESEARCH AT OAK RIDGE NATIONAL LABOYATORY*}

\section{P. M. Hans}

[Abstract of paper presented at the International Evaluation of Operational Practices (IEOP) Group Meeting. Paris, France, November 8-9, 1984]

A summary of the objectives, approaches, results, and underlying research issues of a series of research and development programs conducted by ORNL for the U.S. Nuclear Regulatory Commission is presented to the International Evaluation of Operating Practice (IEOP) group in Paris, France. The IEOP group was formed by EPRI in 1980 to facilitate international exchange of technical information concerned with human performance research in nuclear power plant operation.

The major emphasis of the ORNL program of direct concern to the IEOP group is the methodology that has evolved for control room operator performance measurement using nuclear plant simulators. The methodology includes verified task analysis, automated and manual performance measurement, and quasi-experimental design for experiments in a training environment. Other programs in operator training, human reliability and operator aids are also summarized. -Research sponsored by U.S. Nuclear Regulatory Commis-
sion Office of Nuclear Regulatory Research. 
Section 6

APPLIED RISK AND DECISION ANALYSIS

$\frac{121}{122}$ 


\title{
6.0. INTRODUCTION
}

\author{
G. F. Flanagan
}

The Nuclear Regulatory Commission (NRC) sponsors several risk analysis programs within the division, but because of a lack of sufficient staffing, most of the work is subcontracted to other organizations, with ORNL maintaining a managerial role. One recent major program principally performed at ORNL has been the Pressurized Thermal Shock (PTS) project, in which assessments were made of the vulnerability of three commercial reactor vessels to "through-the-wall" cracking during transients involving rapid cooldown and repressurization. Paper 6.4 below summarizes the objectives of the project, which has now been completed. Documentation of the project is now under way.

A second major project, still in progress, is the development by subcontractor JBF Associates, Inc. of a computer program proposed for use by the NRC Inspection and Enforcement Office. This program (described in Paper 6.1) provides a display of risk information in a rapidly retrievable and organized mode for site and/or regional inspection.

Paper 6.3 is the latest in a series of papers on fire risk models developed for NRC, and Paper 6.2 describes a methodology for estimating health risks created by electricity generation. (The latter work was performed during an earlier reponting period, but the paper was recently given at a conference on the subject.)

Finally, Paper 6.5 deals with a new program for DOE in which the Analytic Hierarchy Process (AHP) is used to prioritize DOE's Liquid Metal Reactor programs.

Other programs that the division oversees but for which no papers were prepared during the reporting period are the Accident Sequence Precursor program, studies of the risks of accidents at $\mathrm{UF}_{6}$ facilities, studies of the risks of recycling contaminated materials, common cause risk analysis, and the In-Plant Reliability Data program, all sponsored by the NRC Office of Research. 
6.1

\section{PRISIM: A PC PROGRAM TO DISPLAY RISK STATUS INFORMATION*}
D. J. Campheil
B. C. Ellisoa'
J. C. Glywat
G. F. Flanagan

(Abstract of paper presented at the American Nuctear Society Topical Meeting on Computer Applications for Nuclear Power Plant Operation and Control, Pasco, WA. September 8-12, 1985)

This paper describes a computer program designed to quickly present important probabilistic risk assessment information to nuclear power industry personnel. The Plant Risk Status Information Management (PRISIM) Program is the first generation of automated information management systems that will allow rapid access to plant-specific risk data. The PRISIM program incorporates three features that make it especially useful to industry personnel:

1. Program software is decision-driven so the user can access only desired information.

2. User-friendly routines are incorporated to allow access or escape to and from any point in the program.

3. Information is presented clearly and concisely using terminology that is familiar to industry personnel.

With the PRISIM system, nuclear industry personnel can use PRA failure models and results to explore safery system, support system, and component contributions to plant risk. The program prescints the major contributions to plant risk completely and quickly and does not require familiarity with probabilistic risk assessment techniques.

- Rescarch sponsored by U.S. Nuclear Regulatory Commission. Division of Risk Analysis and Operations.

$\dagger_{\text {JBF Associates, Inc., Knoxville, TN. }}$

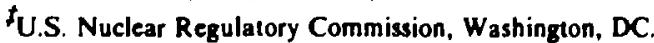

6.2

\section{A METHODOLOGY FOR COMPARING THE HEALTH EFFECTS OF ELECTRICTTY GENERATION FROM LRANIUM AND COAL FUELS*}

\author{
W. R. Rhyne ${ }^{\dagger}$ A. A. El-Bassionit
}

(Abstract of poper presented at EPRI/BNL Workshop on Methods of Assessing Health Impacts, Upton, NY, December 7-8, 1981)

A methodology was developed for comparing the health risks of electricity generation from uranium and coal fuels. The health effects attributable to the construction, operation, and decommissioning of each facility in the two fuel cycles were considered. The methodology is based on defining (1) requirement variables for the materials, energy, etc., (2) effluent variables associated with the requirement variables as well as with the fuel cycle facility operation, and (3) health impact variables for effluents and accidents. The materials, energy, etc., required for construction, operation, and decommissioning of each fuel cycle facility are defined as primary requirement variables. The materials, energy, etc., needed to produce the primary variable are defined as secondary requirement variables. Each requirement variable (primary, secondary, etc.) has associated effluent variables and health impact variables. A diverging chain or tree is formed for each primary variable. Fortunately, most elements reoccur frequently to reduce the level of analysis complexity.

- Research sponsored by the U. S. Nuclear Regulatury Commission, Division of Risk Analysis and Operations.

'H\&R Technical Associates, Inc., Oak Ridge, TN.

${ }^{\prime}$ Division of Risk Analysis and Operations, U. S. Nuclear Regulatory Commission, Washington, DC. 
6.3

\section{MODELING THE DETICTION RATES OF FIRES IN NUCLEAR IOWER PLANTS*}
N. Sis'
G. Ayostolakis'

[Abotract of Trans. Am. Nucl. Sac. 46. 537 (1984)]

A model is developed for detection times for fires in nuclear plants based on the detection mode. Three modes are distinguished in the model: automatic, local, ani remote. The frequency distributions for the inodes are developed using Bayes' Theorem.

-Research sponsored by U.S. Nuclear Regulatory Commission, Office of Probabilistic Fire Risk Analysis.

tUCLA, los Angeles, CA.

6.4

\section{INTEGRATION OF PTS STUDIES TO CALCULATE THROUGH-THE-WALL CRACK PROBABILITIES*}

\section{L. Selby J. D. White'}

(Abstract of paper presented at the Twelfth Water Reactor Safety Information Meeting, National Bureau of Standards, Washington, DC, October 22-26, 1984; Proc. NUREG/CP-C058, Vol. 2, p. 1 (1984)]

This paper describes a NRC-sponsored research project formed to help confirm the technical basis for the proposed Pressurized Thermal Shock (PTS) rule, to aid in the development of guidance for licensee plani-specific PTS analyses, and to examine the effects of proposed corrective measures. The over all objectives of the PTS studies at ORNL are: (1) to provide for each of the three plants consid sred an estimate of the frequency of a PTS-induced through-the-wall crack (TWC); (2) to determine the dominant overcooling sequences, plant fuatures, and operator and control actions, as wel as the important uncertainties, in the PTS risk; and (3) to evaluate the effectiveness of potential corrective measures for reducing the TWC frequencies. ORNL is also to determine what parts of th.e studies might have generic applicability. Thousands of hypothetical overcooling sequences were constructed, full-scale thermal-hydraulics analyses were performed, and probabilistic fracturemechanics calculations were completed.

-Research sponsored by U.S. Nuclear Regulatory Commission, Office of Nectear Regulatory Research.

'Instrumentation and Controls Division.

6.5

\section{PRIORITIZATION OF A LARGE NUMBER OF R \& D PROGRAMS*}

\section{G. Flangan $\quad$ V. R. R. Uppolderi}

(Abstract of paper presented at the Fifth International Conference on Mathematical Modeling, University of California, Berteley, CA, July 29, 1985)

In a research project of interest to DOE, a smali number of characteristics dealing with cost, safety and risks were considered to be important. For each characteristic, a certain number of program elements were compared pairwise, and the relative importance was recorded. Then the Analytic Hierarchy Process (AHP) methods were used to determine the importancr of the program elements.

The main problem is to rank more than 100 programs that fall under each of the characteristics of the project. Since there are too many paired comparisons among the programs, two different approaches were suggested to sbtain a final ranking of the programs. The results of this application will be presented.

"Research sponsored by U.S. Department of Energy. 
Section 7

\section{INFORMATION ANALYSIS AND DATA MANAGEMENT}




\title{
7.0. INTRODUCTION
}

\author{
Betty F. Maskewitz
}

Information analysis and data management, important activities of the division since its inception, comprise a significant fraction of the division's program. Unde: the management umbrella of EPIC (Engineering Physics Information Centers), we currently operate two specialized centers: RSIC (Radiation Shielding Information Center), founded in FY 1963, and TDMC (Technical Data Management Center), founded in 1976. Throughout the years (22), RSIC has served the international scientific community as a technical institute for shielding design and analysis. TDMC performs various tasks in support of NRC (Nuclear Regulatory Commission) programs in technical data management and computer code development, standardization and maintenance.

The Carbon Dioxide Information Center (CDIC), sponsored by the Carbon Dioxide Research Division of DOE's Office of Basic Energy Sciences, was moved out of EPIC facilities and became fully independent of EPIC in FY 1984.

The EPI Centers interact daily with contributors and users through various means of communication. We continuall; seek to improve this interaction through research and experimentation. Some detail follows.

\section{Communications}

Communication is an important element in the functioning of an information center, and new means are being explored to take advantage of new technoiogies. An information center is in daily communication with scientists and engineers who generate information, frequently in foreign countries. It also communicates with users who inquire about information which may help solve their problems or who request specific packages of information. These packages generally consist of computer code systems and/or computer-readable data libraries.

Electronic mail is beginning to take its place alongside the telephone and the conventional mail system. It has the speed advantage of the telephone combined with the noninterruption advantage of mail. EPIC is using and evaluating the two major commercial providers of electronic mail: Western Union EasyLink and MCl Mail. These services also provide access to Telex, which is the main electronic service to foreign countries.

An RSIC account has also been obtained on the Lawrence Livermore National Laboratory Technical Information System (TIS) which provides electronic mail and data base services. In addition 10 TIS user communication, this allows communication on the ARPA network (DARPA/MII,NET) and on BITNET, which links most major universities in the U.S. and in Europe. EPIC staff member accounts on the ORNL-MSR computer (located in the Mathematical Sciences Research Section of the EPM Division) also give access to MILNET and BITNET. 
An EPIC staff member is also leading an effort in the American Nuclear Society (ANS) to establish a communication network in the nuclear industry. In addition to communications, other services may become available, such as computer conferencing, paper review, ordering of publications, industry news, and access to various data bases. A prototype network, called ANS Information Resources Service (ANSIRS), has begun operation.

\section{EPIC and Computer Usage}

Since RSIC began operations in FY 1963, computer codes have normally b/en distributed on reels of magnetic tape. The codes generally were developed for mainframe computers, but recently many are being implemented on smaller systems such as Digital Equipment Corpmation's VAX and personal computers. With the increasing power (greater memory, faster processors, and larger hard disks), personal computers are capable of running many computer codes in the RSIC collection. A iumber of requesters have asked for codes on flexible (floppy) disks with the intention of making them operable on personal computers. In addition, we have obtained several codes written for PCs, and we regularly make these available on floppy disks.

A computer usage survey of readers of the RSIC Newsletter was undertaken and 161 forms were returned. Of these, only three reported no use of PCs. More than half were using IBM PCs or compatibles. We also have standardized on IBM and distribute most diskettes in PC DOS format, although we can also write diskettes in $\mathrm{CP} / \mathrm{M}$ format. It is clear that many useful programs are in use on thesc small computers, and we anticipate acquiring a larg: number of them.

A Data General Eclipse S/130 minicomputer continues to serve the needs of EPIC in cutting time-sharing costs on the central computers and improving data-processing functions. There are mainly two data base management systems on this computer: ADES and STATUS. STATUS handles information on codes acquisition and processing and ADES (Automated Data Entry System) manages requests from institutions or individuals for packaged codes, data and other inf:rmation. Through a remote job entry program, users on the Data General Eclipse can submit jobs to the central network computers (e.g., IBM 3033 and CRAY).

The increased workload for packaging and testing TDMC codes has made it necessary to upgrade the S/130 computer to a Data General MV/4000. This work will be done in FY 1986. The upgrade will allow inore computer memory and disk storage, so the testing of codes and packages for both RSIC and TDMC will be more efficiently done. With the MV/4000, codes written in FORTRAN 77 can be tested. The data bases mentioned above can also run more efficiently without degrading system performance.

RSIC and TDMC are described in greater detail as follows.

\section{Radiation Shielding Information Center (RSIC)}

During this reporting period, RSIC observed its 22 nd year in operation. It continues to be our largest and most widely used information center. Established to promote the exchange of shielding technology, it acts as a resource base for both government and civilian agencies in the United States and in foreign countries and performs such diverse functions as providing bibliographic information; testing, assembling, and distributing computer codes; preparing, testing, and distributing multigroup cross-section libraries, both fine-group and broad-group; collecting and distriburing other types of data bases; holding seminars to educate the shielding community on particular techniques, especially computer-based techniques for solving radiation transport or nuclear data problems; helping to establish shielding benchmark problems and snielding standards; and generally providing problem assistance to requesters. 
The RSIC scope includes the physics of interaction of radiation with matter; radiation production, protection, and transport; radiation detectors and measurements; engineering design techniques; shielding materials properties; computer codes useful in research and design; and nuclear data compilations. The goals of RSIC are to function as a technical institute to provide information (bibliographic and other data, computer codes, technicai advice) upon request; collect, evaluate, enrich, distill, and repackage information to exiend the state of the art, bringing into the public domain technology more usable and more valuable than the sum of the input; and to initiate and effect research and development in appropriate areas of need.

During this reporting period work has continued in the development of fine- and broad-group cross-section data libraries. The 174 neutron, 38 gamma-ray group VITAMIN-E general-purpose cross-section library based on ENDF/B-V now co:! ains 90 materials. It has been used with success for fusion neutronics, fast reactor core and shielding applications, and various other radiation transport calculations within the division. The generation of multigroup data for some of ENDF/B-V evaluations, Revision 2, has also been completed and the resulting data will be released as part of VITAMIN-E.

RSIC has conunued to participate in the work of the Cross Section Evaluation Working Group (CSEWG), with the Shielding Subcommittee presently chaired by the RSIC director. Other staff members are actively involved with the ANS in developing radiation protection and shielding standards and standards for scientific computer programming and its documentation.

In the area of computing technology, RSIC now makes available 685 computer code packages and 115 data packages. The RSIC Newsletter is currently mailed monthly to 1865 people. During FY 1984, more than 3408 letters/telephone calls of requests were processed by RSIC, resulting in 9423 separate activities required to satisfy the requests. A total of 74 persolus came for an orientation visit and/or to use the center's facilities.

It is significant, in terms of reciprocity in international information exchange, that during the 18 months from October 1,1983 to March 31, 1985, 51\% of all incoming computing technology (codes and data) came from foreign coniributors, with Japan, France, and West Germany in the lead. In FY 1984 the contribution of newly developed codes and data compilations by foreign nationals accounted for $70 \%$ of the total. Much of the new technology is of interest to domestic requesters.

The RSIC computer-based information system (SARIS), containing more than 19,900 literature references, is accessible on-line nationally through the DOE RECON syst.m. The seventh indexed bibliography was printed from the data files using computer-based typesetting programs.

The statistical record system (ADES), operable on the EPIC minicomputer (ECLIPSE) and used to compile information about the profile of the RSIC user community, was maintained and modified for more versatility.

\section{Technical Data Management Center (TDMC)}

TDMC was established in July 1976 to serve NRC programs with goals and priorities determined by an advisory committee of NRC technical staff members chaired by the contract monitor within the Division of Technical Information and Document Control of the NRC Office of Administration. Its primary purpose is to support NRC programs by assisting in verification, validation, documentation, and standardization of somputing technology (including codes and other technical data) used in the licensing and regulatnry processes. 
Current work includes the following subtasks: (A) technical data management in the "open code/data package" concept; (B) program enhancement, development of new options and extensions, standardization, and documentation of selected computing technology; $(\sigma)$ technical data generation, validation, and documentation; and (D) publications in support of TDMC tasks and other NRC Technical Data Management Programs.

\section{Subtask A}

Subtask A treats nonshielding methods in several technical areas, including the SCALE (Standardized Computer Analysis for Licensing Evaluation) system. Work was performed in three areas: (1) testing, packaging, dissemination with a mechanism for feedback, and maintenance of stand-alone computer codes associated with SCALE and other code systems; (2) the processing of Release 3 of the SCALE system; and (3) providing information resulting from applications using TDMC-treated codes and data for NRC technical monitors.

Also treated during the reporting period were several code systems (including DEIS and DISPERS) for studies of atmospheric dispersion, meteorological evaluation, and data processing tasks related to routine effluent releases at nuclear power stations. The finished packages are disseminated via RSIC.

\section{Subtesk B}

Subtask B continues to focus on computing technology in the areas of radiclogical assessment, meteorological (atmospheric) and other environmental transport. In addition, the subtask was extended with a major effort devoted to specific code systems of the NRC/NRR/DSI Accident Evaluation Branch. CRAC2 was selected for implementation, verification, documentation, and maintenance as required by AEB staff. A central activity has been the implementation of CRAC2, which is a large-scale nuclear code system, on the NRC Data General Eclipse MV/8000 minicomputer for staff use. As a companion effort, work was done to extend and incorporate an interactive preprocessor for generating CRAC2 input data on an IBM PC. The CRAC2 experience on the MV/8000 has clearly demonstrated the cost-effectiveness of a minicomputer for regulatory applications.

The directory of new codes implemented, tested, and packaged on the MV/8000 with a oneto-one correspondence between those in the RSIC collection anc those available on the NRC in-house system include METD, CRAC2, and XOQDOQ.

Subtasks A and B will again be enlarged in FY 1986 to include acquisition and maintenance of the computing technology designated as reference codes for source term calculations (recommended analytical tools for NRC analyses of accidents severe enough to result in complete core melting).

\section{Subtask C}

Subtask $C$ includes calculations to generate data for the proposed standard on Gamima-Ray Attenuation Coefficients and Buildup Factors for Engineering Materials produced in collaboration with the ANS-6 Standards Subcommittee. Work continues on compiling and analyzing gamma-ray transport data for the proposed ANS-6.4.3 standard. Attention has been concentrated on revising questionable values in the moments method data base and obtaining coefficients for the G-P fitting function which can be used to fit the entire range of energy and atomic number. No other wellknown function can do this. The use of the G-P analysis has demonstrated a new and valuable method for gamma-ray transport data evaluation. The work is being reported in a series of papers 
$133 \mid 13 i$

in Nuclear Science and Engineering. New data for lead and uranium from Japan are being analyzed for possible adoption. Preliminary values for tungsten and tin have been obtained from India. The present version of the text is undergoing revision in preparation for the meeting of the ANS-6.4.3 Working Group in November 1985. Work on this project is proceeding cooperatively in the U.S. at Oak Ridge, Los Alamos, National Bureau of Standards, Bettis Atomic Power Laboratory, GA Technologies, and in India and Japan.

\section{Subtask D}

A draft report entitled "User's Guide for the NRC Staff Version of CRAC2* was prepared and placed in the review process.

An exhibit on TDMC was prepared and manned during the 12th Water Reactor Safety Research Meeting in November 1984.

Assistance was given to regulatory staff in making calculations needed during the preparation of Chapter 6 of NUREG 0956, Reassessment of the Technical Bases for Estimating Source Terms" (draft, July 1985).

Effor is are under way to formally establish TDMC as the official ripository for all NRC computing technology. 
7.1

THE RADIATION SHIELDING INFORMATION CENTER: A SOURCE OF COMPUTER CODES AND DATA FOR DOSIMETRY APPLICATIONS*

\section{$\begin{array}{ll}\text { D. K. Trabey } & \text { B. F. Mastewitz }\end{array}$}

R. W. Ronsiai

[Abstract of Heallh Phys. 47, 198 (1984)]

The Radiation Shielding Information Center (RSIC), established in 1962 to collect, package, analyze, and disseminate information in areas of radiation protection and shielding, has a number of codes and data packages of value for dosimetry. The major activities of the center include: (1) answering technical inquiries on radiatior transport problems, (2) collecting, packaging, testing, and disseminating computing technology ard data libraries, and (3) reviewing literature and operating a computer-based information system containing material pertinent to radiation transport and energy deposition.

The computer codes emphasize methods for solving the Boltzmann transport equation to compute space-dependent fluences, energy spectra, energy depositior., and biological or physical damage. In addition, there are codes which unfold experimental data to give spectra, perform sensitivity analyses, process nuclear cross sections, analyze or predict atmospheric dispersion of radionuclides, perform radiation assessment, and carry out other computations of interest in dosimetry.

The data libraries, available on magnetic tape, include nuclear data, such as radioactive decay information, multigroup cross sections, dose and kerma factors, and damage and neutron activation cross sections. The data libraries are frequently in a format appropriate to the more popular codes such as SAND II and ANISN.

This paper reviews the available RSIC resources with reference to specific applications.

\footnotetext{
- Research sponeored by U.S. Nuclear Regulatory Commie
} sion, Office of Nuclear Regulatory Research.
7.2

\section{THE RADIATION SHIELDING INPORMATION CENTER'S FOREIGN TECHNOLOGY ASSESSMENT - AN AMPORTANT N/. TIONAL ASSET}

\section{B. F. Maskewitz}

(Abstract of presentation given to DOE International Affuirs, Washington, DC, May 24, 1985; two given to DOE/OSTI/TIC, Oak Ridge, TN, Sepeember I2, 1984)

Public Law 94-282 declares Federal Government responsibility to promote systimatic transfer of technology, and special agencies have been established to facilitate this process. The Radiation Shielding Information Center (RSIC) serves this function for its DOE, DNA, and NRC sponsors domestica'ly and internationally in support of the sponsors bilateral and interagency agreements. Through a diligent acquisition effort by staff members, foreign tochnology in areas of radiation production, protection, transport and shielding is well represented in the RSIC collection of isshnical information products. RSIC has promoted international cooperation and reciprocal exchange for more than 20 years. The results of this promotion in FY 1985 are encouraging in that in the first 6 months 10 of the 35 new computer programs and data libraries came from foreign contributors (5 from Japan, 2 from Bulgaria, and I each from Finland, The Netherlands, and France). The technology is evaluated through testing, packaged for transportability and placed in the USA public domain for further assessment and use. Over an 18-month period (FY 1984-1985), 60 separate transmissions of computing technology in RSIC's subject areas were received from foreign nations (Japan 13, France 9, West Germany 7, Bulgaria S, Switzerland 4, Brazil 3, India 3, England 2, Finland 2, Hungary 2, Saudia Arabia 2, and 1 each from Austria, Euratom, East Germany, Italy, The Netherlands, Spain, Taiwan, and USSR). The opportunity to assess the state of the art iuternationally and to have access to the use of interesting foreign methodology is of considerable value 
to the national programmatic interests of RSIC's sponsors.

-Research sponsored by US. Department of Energy.

7.3

\section{ABSTRACTS OF DIGITAL COMPUTER CODE PACKAGES ASSEMBLED BY THE RADIATION SHIELDING INFORMATION CENTER*}

\section{$\begin{array}{ll}\text { B. J. Cartert } & \text { B. F. Mastewitz }\end{array}$}

(Abstract of ORNL/RSIC-13, Vols. I-III/R, April 1985)

This publication, ORNL/RSIC-13, Volumes I-III Revised, has resulted from an internal audit of the first 168 packages of computing technology in the Computer Codes Collection (CCC) of the Radiation Shielding Information Center (RSIC). It replaces the earlier three documents published as single volumes between 1966 and 1972. A significant number of the early code packages were considered to be obsolete and were removed from the collection in the audit process, and the CCC numbers were not reassigned. Others not currently being used by the nuclear R\&D community were retained in the collection to preserve technology not replaced by newer methods or were considered of potential value for reference purposes. Much of the early technology, however, has improved through developer/RSIC/user interaction and continues at the forefront of the advancing state of the art.

- Research sponsorud by U.S. DOE Office of Nuclear Energy. Energy Research, and Defense Programs.

'Franklin Research Center, Philadelphia, PA.
7.4

\section{OVERVIEW OF THE ROLE OF DATA FOR ENERGY SYSTEMS*}

\author{
B. F. Maskewitz \\ J. A. Watts ${ }^{t}$
}

[Abstract of paper presented at 9th International CODATA Conference, Jerusalem, Isreel, June 24-28, 1984; Proe. The Role of Date in Scientific Protress, P. S. Gleeser (ed), p. 279 (1985)]

Growing worldwide societal concerns about critical issues such as the environment and energy sources and supply have created new demands for systematic, authoritative information as a basis for decision making and regulation. Therefore, consideration given to numerical data for energy systems must include data related to the consequences of providing an adequate supply of energy for the societal good. This paper briefly mentions some major data systems and plans for national and international data networking. In addition, other data activities are introduced that are concerned with issues related to environmental hazards due to burning of fossil fuels and to nuclear safety.

-Research sponsorod jointly by U.S. Departmeat of Enerzy and U.S. Nuclear Regulatory Commircion.

'Environmental Sciences Division.

7.5

\section{VITAMIN-E 174n,38g GENERAL-PURPOSE CROSS-SECTION LIBRARY*}

\section{R. W. Roussin J. E. White}

\section{J. L. Bartley}

(Abetract of paper presented at the Inte. tional Conference on Nuclear Data for Basic and Applied Sciences, Santa Fe, NM, May 13-17, 1985)

The "VITAMIN" concept is a somewhat different approach to obtaining appropriate multigroup cross sections for radiation transpon analysis. It was successfully used for VITAMIN-C, which was based on ENDF/B-IV. The ENDF/B-V-based version is called VITAMIN-E. The concept extends the table look-up approach to temperature and resonance 
self-shielding corrections to a wider range of applications. This is done by using many energy groups (174 for neutrons, 38 for photons) and by accommodating photon production and transport. The MINX 15 system was used for neutron processing and LAPHNGAS and SMUG (from the AMPX system) for photon production and transport processing. Applications to which VITAMIN-E have been applied include CSEWG data testing, NEACRP reactor benchmarks, fusion integral experiment analysis and data needs assessment, fusion/fission and accelerator breeder studies, fusion blanket studies, I'WR dosimetry, and air transport.

- Research sponsored jointly by the Defense Nuclear Agency and U.S. DOE OTfice of Fusion Energy.

7.6

\section{VITAMIN-E 174-NEUTRON, 38-GAMMA CROSSSECTION LIBRARY FOR FUSION NEUTRONICS CALCULATIONS*}

\author{
R. W. Roussin J. E. White
}

\section{J. L. Bartley}

[Abstract of Trans. Am. Nucl. Soc. 46, 272 (1984)]

The successful application of VITAMIN-C to fusion neutronics problems led to the development of specifications for an ENDF/B-V-based crosssection library denoted as VITAMIN-E. The early phases of this new cross-section library were focused on materials for fast eactor applications and were applied to benchmark testing of ENDF/B-V.

More recently, requests have been made for additional materials to be added to the basic library for fusion applications. As a consequence, VITAMIN-E now contains 70 materials. As with its predecessor, VITAMIN-E allows great Mexibility to the user to derive problem-dependent sets that can account for resonance shielding and temperature effects. The AMPX system is again employed to perform data manipulations required to create working cross-section dati files.
The energy structure of VITAMIN-E contains 174 neutron and 38 gamma-ray groups and includes the 171 neutron and 36 gamma-ray groups of VITAMIN-C as a subset. The ENDF/B-V Special Purpose Dosimetry, Activation, and Gas Production Files have also been processed into the VITAMIN-E neutron group structure.

The initial release of the VITAMIN-E crosssestion library will be in AMPX format. Versions in CCCC and MATXS formats will be made available at a later time. VITAMIN-E and the supporting AMPX modules can be obtained from the Radiation Shielding Information Center at Oak Ridge National Laboratory. The library is also available on the National Magnetic Fusion Energy Computing Center at Lawrence Livermore National Laboratory.

-Research sporsored by the Defense Nucleas Ageacy and U.S. DOF Sffice of Fusion Energy and Office of Reacior Research and Techoology.

7.7

\section{BEGINNER'S GUIDE TO BLOCKED RECORDS ON MAGNETIC TAPE*}

\section{B. L. Kirt}

(Abstract of ORNL/TM-9250, September 1984)

The subject of this report is the usage of magnetic tape for data storage. The appendix listings are intended to guide users in choosing the best possible parameters when blocking data of record lengths 80 or 133 bytes. Only fixed block records are of interest here. The formulas listed in the report are also applicable to records having varied lengths. The use of 80 and 133 bytes is dictated by their popularity.

-Research sponeored by U.S. Depart ment of Energy 
7.0

\section{SYSTEMS GUIDE TO MCNP (MONTE CARLO NEUTRON AND PHOTON TRANSPORT CODE)*}

\section{B. L. Kirt J. T. Weat ${ }^{t}$}

(Abstract of ORNL/TM-9123, June 1984)

The subject of this report is the implementation of the Los Alamos National Laboratory Monte Carlo Neutron and Photon Transport Code - Version 3 (MCNP) on the different types of computer systems, especially the IBM MVS system. The report supplements the documentation of the RSIC computer code package CCC-20r, MCNP. Details of the procedure to follow in executing MCNP on the IBM computers, either in batch mode or interactive mode, are provided.

- Research sponsored by U.S. DOE OFTrce of Fusion Exergy, U.S. DOE Office of Military Applications and Defense Nuclear Agency.

flos Alamos National Laboratory, Los Alamos, NM.

7.9

\section{NRC TECHNICAL DATA MANAGEMENT CENTER (TDMC)*}

\section{J. E. White R. W. Ronsain}

\section{B. F. Maskewitz}

(Abatract of poster presentation at the Twelfth Water Reactor Safety Informaticn Meeting, National Bureau of Standarda, Washington, DC, October 22-26, 1984)

TDMC is sponsored by the Nuclear Regulatory Commission (NRC) to provide technical information and technology support to assure proper documentation and modification of the analytical work of the NRC staff. Established in 1976 at the Oak Ridge National Laboratory (ORNL), TDMC is monitored by the Technical Information and Documentation Control (TIDC) Division of the Office of Admiristration (ADM). Services available to all NRC technical divisions: technical data management in the "open code/data package concept"; computer program enhancement, standardization, verification and documentation; data generation, validation, and documentation; installation and maintenance of computing technology on the NRC Data General MV/8000 super minicomputer and other selected computers; and pablication support of TDMC tasks and other NRC technical data management programs. TDMC collaborates with the NRC contract monitor and technical staff members to select pertinent computing technology for treatment. Recent examples include LADTAP-II, XOQDOQ, LPGS, and TACT5. Current processing includes CRAC2, MESODIF-II, and GENDOS. Dissemination and collection of feedback is provided. A prime example of this concept is the SCALE (Standardized Computer Analyses for Licensing Evaluation) system of the Office of Nuclear Material Safety and Safeguards (NMSS). The latest. version, SCALE-3, will soon be submitted to TDMC by the ORNL developers.

- Research spopsored by U.S. Nuclear Regulatory Commistion Ortice of Administration.

7.10

\section{USER'S GUIDE FOR THE NRC STAFF VERSION OF CRAC2.}

\section{J. E. White R. W. Romatin}

(Abstract of peper presented at a Workshop on the Application of a Minicomputet for Large-Scale Nuclear Computation, NRC, Washington, DC, February 7-8, 1985)

The CRAC2 computer code has been implemented for use on the Nuclear Regulatory Commission Data General MV/8000 computer system. Input preparation is facilitated through the use of an interactive computer program which operates on an IBM personal computer. The resulting CRAC2 input deck is transmitted to the MV/ 8000 by using an error-free file transfer mechanism. To facilitate the use of CRAC2 at NRC, relevant background material on input requirements and model descriptions was extracted from three reports - "Calculations of Reactor Accident Consequences," Version 2, NUREG/CR-2326 (SANDB1-1994), "CRAC2 
Model Descriptions," NUREG/CR-2552 (SAND82-0342), and "CRAC Calculations for Accident Sections of Environmental Statements," NUREG/CR-2901 (SAND82-1693). When this background material is combined with instructions on the input processor, a self-contained guide for preparing CRAC2 input data with an orientation towards applications on the MV/8000 is provided.

Availability of CRAC2 on the MV/8000 gives the Accident Evaluation Branch an inexpensive alternative to the costly calculations performed on a mainframe in support of environmental statements.

- Research sponsored by U.S. Nuclear Regulatory Commission Office of Administration.

7.11

\section{EFFECT OF FLUORESCENCE, BREMSSTRAHLUNG, AND ANNIHILATION \\ RADIATION ON THE SPECTRA AND ENERGY DEPOSITION OF GAMMA RAYS IN BULK MEDIA*}
K. V. Subbaiah ${ }^{\dagger}$
D. V. Gopinath ${ }^{\dagger}$
A. Natarajan ${ }^{t}$
D. K. Trubey

[Abstract of Nucl. Sci. Eng. 81, 172 (1982)]

The contribution of bremsstrahlung, annihilation, and fluorescence radiation to the spectra of scattered gamma radiation and to the dose buildup factors has been investigated as a function of source energy, atomic number, and sample thickness over an energy range of 0.1 to $8 \mathrm{MeV}$. The computations were performed with the onedimensional transport code ASFIT modified to take into account all the secondary radiations. The required mathematical formulations, along with representative results obtained for uranium, lead, iron, and water, typifying materials of very high, high, medium, and low atomic number are respectively presented and discussed.

A noticeable effect of including bremsstrahlung sources is the general softening of the scattered radiation spectra inside the medium and at the exit. This effect is more pronounced in materials of high atomic number. The bremsstrahlung contribution is seen most proininently in the reflection spectra above 0.511

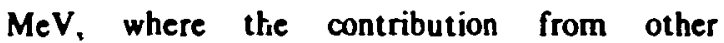
processes is insignificant. The effect of annihilation radiation is significant in the region between $0.511 \mathrm{MeV}$ and the $K$ edge, below which the effects of fluorescence radiation overshadow all others. Peaks and discontinuities characteristic of single scatterings of these radiations are seen in the reflection spectra, gradually disappearing with depth in the medium.

The effect of fluorescence on the dose buildup factor is spectacular for source energies close to the $K$ edge and falls off rapidly thereafter. The impact of bremsstrahlung, on the other hand, steadily rises with source energy. The influence of annihilation radiation is comparatively modest and is significant only for systems of intermediate atomic numbers.

- Research sponsored by U.S. Nuclear Regulatory Commission Orfice of Administration.

'Reactor Research Centre, Safety Research Laboratory, Kalpakkam, India.

7.12

\section{CARBON DIOXIDE INFORMATION CENTER (CDIC): A PROGRESS REPORT FOR THE PERIOD JANUARY 1982 - SEPTEMBER 1983*}
B. F. Maskewitz
J. A. Watts ${ }^{t}$
C. R. Weisbin
L. J. Allisont
B. F. Jacobst
H. A. Pfuderert

(Abstract of ORNL/TM-9350, October 1984)

The Carbon Dioxide Research Division (CDRD) of the Depariment of Energy's (DOE) Orfice of Basic Energy Sciences established a Carbon Dioxide Information Center (CDIC) at the Oak Ridge National Laboratory (ORNL) in January 1982 to support the nation's carbon dioxide-climate research effort by providing a focal point for the compilation and distribution of $\mathrm{CO}_{2}$-related information under systematic quality control. This informal report documents the establishment and experience of the center duriag 
the period January 1, 1982 - September 30, 1983. Progress on the activities and accomplishments by the center are discussed using specific examples to illustrate the concept and develop- ment of a specialized informatiun analysis center.

- Research sponsored by U.S. DOE Office of Busic Energy Sciences.

Environmental Sciences Division.

Information Division. 
Section 8

MATHEMATICAL SCIENCES 


\title{
8.0. INTRODUCTION
}

\author{
R. C. Ward
}

The Mathematical Sciences Section joined the division early in 1984, prompting the change in name from the Engineering Physics Division to the Engineering Physics and Mathematics Division. The section itself i; not new to the Laboratory, however, since this is the first time the section's work is included in the progress report, it seems appropriate to review some of its history.

Mathematical sciences research at ORNL had its beginnings in the Mathematics Panel organized by Professor Alston S. Householder in 1948. Dr. Householder attracted a number of mathematicians and statisticians to his staff, and by 1956 he had formed mathematics and statistics sections, which together with an expanding group of computer scientists became the Mathematics Division of the Laboratory in 1962. Dr. Householder led the Mathematics Division until he retired in 1969. Dr. Harvey P. Carter then became Director of the Mathematics Division, and in 1973 organized the Computer Sciences Division, responsible for a broad spectrum of duties ranging from fundamental research in the mathematical sciences to the maintenance of the extensive computer facilities at the Oak Ridge DOE installation. The research arm of the division was located in the Mathematics and Statistics Research Department of the Computer Sciences Division until February, 1984, when it became a section of the new Engineering Physics and Mathematics Division of Oak Ridge National Laboratory.

The Mathematical Sciences Section has three main objectives:

1. To perform. research in applied mathematics, statistics, and computer science which is germane to the solution of the nation's energy problems,

2. To consult and collaborate with other scientists workin $z$ on these problems, and

3. To provide for technical development and growth of its personnel in advanced and leading- - dge mathematical, statistical and computer science technology.

Typically, ezch section staff member engages in both research and consulting; however, the percentages of tirne staff niembers devote to those activities may vary with time and individuals. Almost all of th: researchers are actively involved in providing high-level consulting services to scientists and ençineers at ORNL. This consulting activity provides motivation for research ideas and a source of e::cellent test cases for evaluating research results.

During this reporting period, approximately forty-five percent of the staff support came from basic research proposals generated by the staff; the remaining fifty-five per cent came from consulting activities. Sronsors of our basic research were the DOE Applied Mathematical Sciences Program, the DOE Office of Health and Environmental Research, the AFOSR Computational Mathematics Program, the NASA Marshall Space Flight Center's Materials Processing Program. and the Oak Ridge National Laboratory. ORNL divisions, in addition to our own, which utilized 
our expertise at a significant level were the Biology, Energy, Engineering Technology, Environmental Sciences, Health and Safety Research, and Solid State Divisions. Other Martin Marietta Energy Systems organizations which relied heavily on our knowledge in the mathematical sciences were the Computing and Telecommunications Division, the Y-12 Plant's Quality Division, and the Separation Systems Division.

The section underwent several organizational changes during this reporting period. The two statistics groups were combined, and the mathematics group was split into a mathematics group and a computer science group. This latter change was in response to our recent emphasis on research in parallel computing.

In support of its computational and research needs, the section has utilized a variety of computers. It has continued to use its Research Computing Facility consisting of a VAX 11/780 and a newly acquired Intel iPSC/d6 hypercube parallel computer. The VAX computer has served inhouse computing and communications needs and is also the ORNL host for the Defense Data Network (ARPANET/MILNET). The hypercube is a state-of-the-art parallel computer having 64 asynchronous processors; it serves as a research tool in the effort to explore computational algorithms for parallel computers. Network access to the Argonne National Laboratory was utilized to access the HEP parallel computer manufactured by the Denelcor Corporation. To meet its substantial computing needs, the section has accessed via networks CRAY and CYBER computers located at various sites around the nation.

\section{Applied Mathematics}

Research and consulting in moving-boundary and phase-change problems continued to be the dominant area of activity of the Mathematics Group. Significant results were obtained in the course of developing models for the solidification of the alloy system mercury-cadmium-telluride in work sponsored both by the DOE Applied Mathematical Sciences program and by NASA. The results were in both theoretical and numerical aspects of the extremely nonlinear system of equations arising from the solidification process, and in the additional physical realities present in it. These included strong constitutional supercooling of the liquid phase and subsequent heterogeneous nucleation which results in buoyant, growing solid bodies. The buoyancy of the resulting solids is due to the low mercury content of the solid phase when in equilibrium with the liquid phase. In our efforts to better understand the process being simulated, collaboration was established with Max Morris of the Statistics Group, who applied a technique of Bayesian analysis in order to obtain quantitative relations between system variables.

In FY84 our group initiated a study of phase-change processes under hyperbolic heat transfer in response to the infinite speed of heat propagation as predicted by the parabolic model. This model of heat transfer appears to be required for rapid and high heat transfer processes due, for instance, to rapid pulsed laser-driven heat injection. Substantial progress in this area has resulted from the observation by Dr. James Greenberg of the DOE Applied Mathematical Sciences Program that at the phase-change front one might 6 ..ve discontinuous temperatures. The possibility of physically seeing such discontinuities has b $\mathrm{c..}$ raised in subsequent discussions with physicists working in this area. Progress has been made in both mathematical theory (well-posedneas, maximum principles, etc.) and numerical simulation of the process.

Additional progress in phase-change modeling was manifested by the development of a new numerical approach to the Stefan problem, based on the Keller Box Scheme, the derivation of a number of tools of vectorization for phase-change p:ocesses, and the development and study of solidification of supercooled liquids with the incorporation of the Gibbs-Thompeon relation at the front. 
Progress in other areas represented by the Mathematics Group took place in our research on disordered systems and in electrochemical machining, which is being done jointly with staff members of the Y-12 plant.

Our group was considerably strengthened by the addition of Dr. Skip Thompson, who assumed leadership of the Scientific Software Consulting Project in January, 1985. Advances in this project were made in the areas of delivering requested software to users via networks and in a number of studies on ordinary differential equation solvers.

\section{Compater Science}

The traditional research emphasis of the Computer Science Group has been the development of numerical algorithms for matrix computations, particularly those involving large sparse matrices. Although some work in this area has continued, we have also begun a major new initiative in the area of parallel computing, and this was the primary emphasis of research in Computer Science during the reporting period. Major accomplishments included:

1. Acquisition of an Intel iPSC/d6 hypercube parallel computer having 64 processors and 32 megabytes of memory. This machine was among the first such equipment produced and serves as our primary experimental test-bed for our parallel computing research.

2. Development of a parallel processor simulator for our $\operatorname{Vax} 11 / 780$. This simulator enables algorithm development and debugging for various message-passing, local-memory multiprocessor architectures (particularly the hypercube) and allows the tuning of critical parameters to model specific machines more accurately.

3. Development of parallel algorithms for various important problems in numerical linear algebra (for both dense and sparse matrices), including the Cholesky, LU, and QR factorizations. Some of these algorithms are specifically tailured to the hypercube, while others are more broadly applicable to a variety of both local-memory and shared-memory multiprocessors.

4. Investigation into the feasibility of both physical and logical interconnection of various computer networks, including hardware compatibility and integration of diverse network protocols.

These activities have resulted in a greatly increased external visibility for our research program in parallel computing. We are now nationally and internationally recognized as leaders in this emerging new area of scientific computation.

Based on our initial research in parallel computing. we have made a number of important observations. We have shown that for direct methods of matrix factorization fairly high processor utilization $(\sim 85 \%)$ can be attained by reasonably straightforward adaptation of serial algorithms and that even higher processor utilization $(\sim 95 \%)$ can be attained through careful tuning of the code. We have observed that the ratio of communication speed to computation speed is an important factor in determining the effectiveness of any distributed system and that a value near $I$ is most appropriate. We have also observed that attaining good parallel efficiency depends on the problem size growing as the number of processors employed grows. We have studied a num 'er of ways of mapping matrices onto processor arrays and have shown that the block mapping used in previous algorithms is inferior to wrap and reflection mappings.

Progress was also made in the more traditional areas of computational wothematics during the reporting period. These included the development of numerical algorithms for computing the singular value decomposition of a product of matrices, computing a sparse basis for the null space of a 
sparse matrix, implementing the force method for structural analysis, and solving various problems regarding $\mathbf{M}$-matrices arising in Markov chains and queuing networks.

\section{Statistics}

Research in the Statistics Group has been concentrated in the areas of biostatistici and statistical methods. The objective of the biostatistics research is to upgrade the statistical methodology for designing experiments and analyzing data related to health effects (including mutagenicity and carcinogenicity) of chemicals and irradiation. The research is concentrated in the following areas: design and analysis of survival/sacrifice experiments for assessing effects of chemical or radioactive insulis to animals; and the analysis of bioassays for the determination of dose-effect curves.

Significant accomplishments included work on:

1. The mathematical characterization of the structure of estimation problems associated with sigmoid dose-response curves, which are used on applications such as assessing the toxic or carcinogenic nature of low-dose exposure to potentic 'ly hazardous substances;

2. The design of optimal sacrifice strategies in experi zents to study disease incidence and progression in animals exposed to radiation or chemicals; and

3. The development of regression methods specifically for discrete response variables, as opposed to continuous variables.

The statistical methods research effort is divided between computational statistics (CS) and analysis of !arge data sets (ALDS) research projects. The CS project includes work in computeraided experimental design, algorithms for computing distributional properties of estimators and est statistics, iteratively reweighted least squares algorithms, computational aspects of large-scale regression problems requiring sparse matrix technology, and algorithms for computing Bayesian posterior distributions. The ALDS project focuses on the evaluation of the effect of data transformation on misclassification probabilities in multivariate discriminant analysis; development of Bayesian variable selection methods; and development of methods to analyze data arising from imperfect sampling frames.

Major accomplishments include:

1. Construction of experimental designs that are good with respect to multiple criteria, i.e., precise estimation of the model parameters as well as the ability to detect model inadequacy;

2. Construction of supersaturated experimental designs for linear statistical models, a class of designs of interest when there are a large number of controllable variables;

3. Development of computational methods for obtaining distributional properties of statistical estimators for the Gamma distribution used frequently in reliability studies; and

4. Development of computer codes to calculate posterior distributions of interest in Bayesian variable selection.

The group has collaborated with research and development personnel in most of the divisions of the Oak Ridge National Laboratory and many of the divisions of the Y-12 Plant, Oak Ridge Gaseous Diffusion Plant, and the Oak Ridge Associated Universities in order to assist in the statistical design and interpretation of experiments and investigations during the reporting period.

Areas of application of statistical methods during the reporting period have included energy modeling, investigation of properti:s of materials, safeguard studies, reactor safety, validation of 


\section{$147 \mid 145$}

computer models, reliability, planning investigations which require statistical sampling, quality assurance and process control, epidemiological studies, tests on potential carcinogens and mutagens, effects of energy-related byproducts on the environment, risk analysis, bioassay, energy consumption estimation, survival/sacrifice experiments, and estimation of disease incidenre in small animals. In addition, the group has operated the Statistical Computing Office, which provides general and computational assistance on the use of SAS and other software for statistical computations. 


\section{Applied Mathematics}

8.1

\section{A NEW NUMERICAL METHOD FOR PHASE CHANGE PROBLEMS*}

\section{Alexiades}

(Abstract of paper presented at the 2Ist Annual Meeting of the Society for Engineering Science, Blacksburg, VA, October 17. 1984)

Melting and solidification processes give rise to nonlinear problems for parabolic PDEs with unknown (moving) boundaries.

After an overview of existing numerical approaches and their shortcomings, a new scheme will be presented, designed to overcome these shortcomings. The new approach is based on an implicit in time finite-difference discretization of the enthalpy (weak solution) formulation of the problem. It allows the tracking of even multiple fronts and mushy regions in a self-consistert manner, based entirely on energy conservation (without relying on Stefan type interlace conditions).

Numerical examples will be presented.

- Research sponsored by the Rpplied Mathematical Sciences Research Program, U.S. DOE Office of Energy Research.

\section{2}

\section{ALLOY SOLIDIFICATION WITH CONSTITUTIONAL SUPERCOOLING*}

\section{Alexizdes}

(Abstract of paper presented at the University of Thessaloniki, Greece, May 16 28, 1985)

The solidification of a binary alloy is governed by simultaneous conduction of heat and diffusion of solute. In addition, the phase-change temperature depends on the concentration as dictated by the phase diagram of the alloy. As a result, con- stitutional supercooling arises when the local state of the material lies between the liquidus and solidus curves of the phase diagram. We describe a general macroscopic model of (transient) solidification based directly on fundamental thermodynamic principles.

The model is globally formulated as a pair of conservation laws valid over the whole region occupied by the alloy in a weak (distributional) sense and it is general enough to allow thermal-diffusive cross effects as well as anisotropic thermodunamic and transport coefficients, which may depend on both concentration and temperature.

A numerical implementation and results for a mercury-cadmium-telluride alloy will also be discussed.

- Research sponsored by the Applied Mathematical Sciences Research Program, U.S. DOE Office of Energy Research.

8.3

\section{REMARKS AND QUESTIONS ABOUT HYPERBOLIC STEFAN PROBLEMS*}

\section{Alexiades}

(Abstract of paper presented at the Unjversity of Torino, Italy. May 1985)

The study of phase change processes governed by hyperbolic heat transfer is at an embryonic stage. We raise some of the relevant open questions and make some remarks on the formulation and qualitative behavior of hyperbolic Stefan problems. In particular, we present a simple one-phase problem with explicit solution and study its behavior. We also describe a weak (enthalpy) formulation for two-phase problems and some numerical experiments based on it.

- Research sponsored by the Applied Mathematical Sciences Research Program, U.S. DOE Office of Energy Research. 
8.4

\section{THE ORNL BINARY ALLOY SOLIDIFICATION MODEL.}

\section{Alexiades}

(Abstract of paper presented at a Workshop on Alloy Solidification, Floreace, Italy, May 1985)

The solidification of a binary alloy is governed hy simultaneous conduction of heat and diffusion of solute. In addition, the phase-change temperature depends on the concentration as dictated by the phase diagram of the alloy. As a result, con stitutional supercooling arises when the local state of the material lies between the liquidus and solidus curves of the phase diagram. We describe a general macroscopic model of (transient) solidification based directly on fundamental thermodynamic principles.

The model is globally formulated as a pair of conservation laws valid over the whole region occupied by the alloy in a weak (distributional) sense and it is general enough to allow thermaldiffusive cross effects as well as anisotropic thermodynamic and transport coefficients, which may depend on both concentration and temperature.

A numerical implementation and results for a mercury-cadmium-telluride alloy will also be discussed.

- Research sponsored by the Applied Mathematical Sciences Research Program, U.S. DOE Office of Energy Research.

8.5

\section{MINIMIZERS OF THE FREE ENERGY IN THE THERMODYNAMIC THEORY OF FLUID INTERFACES*}

\section{Alexiades}

(Abstract of paper presented at the University of Torino. Italy, and the University of Crete, Greece. May 1985)

In the classical variational Van der WaalsCahn-Hilliard theory of fluid interfaces, the stable density profiles are minimizers of the free energy which is allowed to depend not only on the density but also on the density gradient. For a one-dimensional fluid interface we prove that only the monctone profiles are minimizers (this is a problem in the Calculus of Variations over infinite intervals). Thus we make rigorous certain assertions existing in the physical literature and establish the inability of the variational theory to explain observed periodic structures (as in spinodal decomposition). Finally, we outline a generalized continuum thermodynamic theory of E. C. Aifantis, which contains the variational theory as a special case and does not necessarily suffer from such limitations.

- Psea $h$ sponsored by the University of Tennessec and Oak Ridge . vational Laboratory.

\section{6}

\section{A GENERAL MODEL OF BINARY ALLOY SOLIDIFICATION*}

\author{
V. Alexiades D. G. Wilson
}

\section{A. D. Solowon}

(Abstract of paper presented at a Colloquium, NASA, Marshall Space Flight Center, Huntsville, AL, May 1984)

A macroscopic mathematical model is constructed describing the evolution of the phases of a binary lloy or mi-ture undergoing solidification under the action of simultaneous conduction of heat and diffusion of solute. The formulation is global, in the form of a pair of conservation laws valid over the whole region occupied by the alloy in a weak (distributional) sense. Thus it is especially convenient for numerical soluticn since it does not require tracking of the interface, which, in fact, may develop into a "mushy zone."

- Research sponsored by the Applied Mathematical Sciences Research Program, U.S. DOE Office of Energy Research. 
8.7

\section{ON THE THERMODYNAMIC THEORY OF FLUID INTERFACES: INFINITE INTERVALS, EQUILIBRIUM SOLUTIONS, AND MINIMIZERS*}

\author{
V. Alexiades $\quad$ E. C. Aifantis ${ }^{t}$
}

[Abstract of J. Colloidal and Interface Sci. (in press)]

We outline a thermodynamic theory for onedimensional fluid interfaces and compare our findings with the classical results of the variational Van der Waals-Cahn-Hilliard approach. After establishing necessary and sufficient conditions for their equivalence, we list all types of possible solutions giving the structure of the density profile in an infinite interval. Then we examine the stability of these solutions, strictly within a variational thermodynamic context and prove that transitions are minimizers, but reversals and oscillations are not. To the best of our knowledge, this is the first proof available for this old problem. It substantiates previous intuitive statements and makes rigorous certain mathematical assertions existing in the physical literature.

- Research sponsored by the University of Tennessee and Oak Ridge National Laboratory.

'Michigan Technological University, Houghton, MI.

\section{8}

\section{NUMERICAL SIMULATION OF A HgCdTe SOLIDIFICATION PROCESS*}

\author{
V. Alexiades G. A. Geist
}

\section{A. D. Solomon}

(Abstract of ORNL-6127. August 1985)

The solidification of a cylindrical ingot of mercury-cadnilum-telluride is modeled taking i.nto account both heat conduction and solute diffusion. Values of the relevant thermophysical parameters of the pseudo-binary $\mathrm{HgTe}-\mathrm{CdTe}$ are compiled. The model is implemented numerically by a finite-difference discretization and results of the sinulation of a freezing experiment are reported.

- Research sponsored by the Applied Mathernatical Sciences Research Program. U.S. DOE Office of Energy Research and by the Microgravity Science and Applications Division of the National Aeronautics and Space Administration.
8.9

\section{MACROSCOPIC GLOBAL MODELING OF BINARY ALLOY SOLIDIFICATION PROCESSES*}

\section{Alexiades D. G. Wilson}

\author{
A. D. Solomen
}

[Abstract of Q. Appl. Math 43(2), 143 (1985)]

A macroscopic mathematical model is constructed describing the evolution of the phases of a binary alloy or mixture undergoing solidification under the action of simultaneous conduction of heat and diffusion of solute. The formulation is global, in the form of a pair of conservation laws valid over the whole region occupied by the alloy in a weak (distributional) sense. Thus it is especially convenient for numerical solution since it does not require tracking of the interface, which, in fact, may develop into a "mushy zone."

-Research sponsored by the Applied Mathematical Sciences Reseasch Program, U.S. DOE Offiec of Energy Research.

8.10

\section{PCMSOLI - A DIRECT GAIN SOLAR SIMULATION CODE WITH PHASE CHANGE MATERIALS*}
G. A. Geist
A. D. Solomon
C. A. Serbin
J. Tomlinson'

(Abstract of paper presented at the Desert Research Institute, Israel, July 10, 1984)

In order to study the optimum properties, utilization and economics si phase change materials $(\mathrm{PCM})$ in a direct gain passive solar structure, a computer simulation. PCMSOLI, has been developed. This program, modeling a one-room home, permits the incorporation of PCM's and/or other high thermal mass materials in the walls. The theory and basic relations used in the code are given. Some sample runs are discussed.

\footnotetext{
- Research sponsored by Thermal Energy Storage Program. U.S. DOF Office of Energy Research.

${ }^{\prime}$ Engineering Technology Division.
} 
8.11

\section{SELF-CONSISTENT APPROXIMATIONS FOR RANDOM ALLOYS*}

\section{J. Gray}

(Abstrect of paper presented at ORAU Traveling Lecture. Williamsburg. VA, April 27, 1984)

The Coherent Potential Approximation (CPA) has long been recognized as the best single-sile approximation for calculating the average Green's function of a random alloy. Self-consistent methods intended to generalize the CPA so as to include either cluster scattering or off-diagonal disorder have been, until recently, plagued by serious problems (most notably, non-analytic behavior). This situation has been resolved by the Traveling Cluster Approximation (TCA) and Augmented Space methods. These developments will be described, and related work pertaining to amorphous systems will be mentioned.

-Research sponsored by Division of Material Sciences and the Applied Mathematical Sciences Research Program, U.S. DOE Office of Energy Research.

\subsection{2}

\section{A FRACTAL MODEL FOR CHARGE DIFFUSION ACROSS A ROUGH INTERFACE*}

\section{J. Gray S. H. Liv't T. Kaplan ${ }^{\prime}$}

(Abstract of paper presented at the NATO Advanced Study Institute on Scaling Phenomena in Disordered Syntems, Geilo, Norway, April 10-21, 1985)

A fractal model is proposed for a rough interface hetween two materials of very different conductivities, e.g., an electrode and an electrolyte. The fractal mndel is examined for two cases: one where the scaling and branching at each successive stage is fixed and the other where both the scaling and branching are treated as random variables. In both cases the mode has the property of the so-called constant-phase-angle element, i.e., a passive circuit element whose complex impedance has a power law singularity at low frequencies.
The exponent of the frequency dependence is a function of the disorder and is related to the fractal dimension.

- Research sponsored by the Applied Mathematical Sciences Research Program and by the Division of Material Sciences, U.S. DOE Ofince of Energy Research.

'Solid State Division.

\subsection{3}

\section{SIMULATION OF THE ELECTROCHEMICAL MACHINING PROCESS*}

\author{
L. J. Gray C. A. Sertio \\ W. C. Dietrich
}

(Abstract of ORNL-6054, November 1984)

An algorithm for simulating a twodimensional electrochemical machining process is presented. A major part of the calculation, the solution of Laplace's equation, is accomplished by a boundary element method based upon Green's formula. Polarization, in the form of the Tafel equation, is included in the model. The computer program is discussed in detail.

-Research sponsored by the U.S. Departmeat of Energy.

'Development Division, Y-12 Plant.

\subsection{4}

\section{SELF-CONSISTENT APPROXIMATION FOR MUFFIN-TIN MODELS OF RANDOM SUBSTITUTIONAL ALLOYS WITH ENVIRONMENTAL DISORDER*}

\section{T. Kaplan' $\quad$ L. J. Gray}

[Abstract of Phys. Rev. B 2916), 3684 (1984)]

The self-consistent approximation of Kaplan, Leath, Gray, and Diehl is applied to models for substitutional random alloys with muffin-tin potentials. The particular advantage of this approximation is that, in addition to including cluster scattering, the muffin-tin potentials in the 
alloy ean vepend on the occupation of the surrounding sites (i.e., environmental disorder is included).

- Research sponsored by the Division of Material Sciences. U.S. DOE Mfroc ef Energy Research.

'Solid State Division.

\section{ROLE OF MATHEMATICS AND STATISTICS RESEARCH SECTION OF OAK RIDGE NATIONAL LABORATORY*}

\section{A. Serbin}

(Abstract of paper presented at Society for Industrial and Applied Matbematic National Moeting. University of Washington, Seattke, WA, July 16-20, 1984)

Most of the mathematicians employed by the ORNL are found organizationally in the Mathematics and Statistics Research (MSR) Section of the Engineering Physics and Mathematics Division. MSR is responsible for conducting research in the areas of mathematics, statistics, and computer science; for providing consultation services for other researchers and investigators; and for providing training in mathematical, statistical, and numerical analysis. Current areas of research interest in MSR include computational statistics, numerical linear algebra, moving boundary problems, algorithms for parallel computers, and biometrics. Research results from each of these areas will be illustrated. Consultations typically involve analytical and numerical modeling and analysis with the problems coming from a wide range of application areas such as epidemiology, nuclear safeguards, control theory, and energy stolage. Examples of this mathematical collaboration will also be presented.

- Research sponsored by Oak Ridge National Laboratory.
8.16

\section{THE MATHEMATICS OF ARTIFICIAL INTELLIGENCE*}

\author{
A. D. Solomon
}

[Abstract of ORNL Review 17, 28 (1984)]

We present a survey of the main ideas of artificial intelligence, discussing the role of "expert" opinion, knowledge, intelligence, and logic. Examples are given of bow the techniques of artificial intelligence might be used in a variety of fields, including robotics and simulation.

- Research sponsored by Oak Ridge National Laboratory.

8.17

\section{APPROXIMATION METHODS FOR PHASE CHANGE PROBLEMS*}

\section{A. D. Solomon}

(Abstract of paper presented at Tel Aviv University. Tel Aviv, Israel. Junc 18, 1984)

We review the key numerical and analytical approximation methods for phase change problems. These include the front tracking and weak solution numerical methods, and the Megerlin and Goodman analytical approaches. An example is given, based on the outward solidification of a supercooled liquid drop.

-Research sponsored by the Applied Mathematical Sciences Research Program, U.S. DOE Office of Energy Research.

\subsection{8}

\section{MATHEMATICAL METHODS IN LATENT HEAT STORAGE*}

\section{A. D. Solowon}

(Abstract of paper presented at Technion-Israel Institule of Technology, Haifa, Israel, June 26, 1984)

We discuss the physical origins and approaches to fatent heat storage modeling. 
These include numerical and analytical approaches, as well as approaches to supercooling solidification, mixture solidification and melting. and layered structures of different phase change materials.

- Research sponsored by the Thermal Energy Storage Program, U.S. DOE Office of Energy Research.

\subsection{9}

\section{PITTALLS OF COMPUTER SIMULATION*}

\section{A. D. Solomon}

(Abstract of paper presented at Ben-Gurion University, Beersheva, Israel, July 2-3, 1984)

We describe a uumber of cases where problems have arisen in attempts to simulate simple heat transfer and phase change processes. Included are problems of well-posedness of the underlying mathematical questions (e.g., supercooling), convergence (e.g., of implicit solutions to enthalpy-based methods), and error control (e.g., how many nodes should we use?).

- Rescarch sponsored by Appliod Mathematical Sciences Research Program, U.S. DOE Office of Energy Research.

\subsection{0}

\section{NUMERICAL METHODS FOR SOLVING STEFAN-TYPE PROBLEMS*}

\section{A. D. Solomon}

[Abstract of Rev. Mechanica (in press)]

We discuss numerical methods for simulating phase change processes, focusing on enthalpy methods. We then look at the necessity of using enthalpy methods for certain phase change processes in materials science. We close with a brief list of some of the challenges facing us in this area.

\footnotetext{
- Research sponsored by Applied Mathematics Sciences Research Program, U.S. DOE Office of Energy Research.
}

8.21

\section{WHAT IS MATHEMATICS TO US?*}

\author{
A. D. Solomon
}

(Abstract of a talk presented at Oakland University. Roctrester, MI, May 22, 1985)

Mathematics and its tools form the basis for every activity associated with the current period of rapid technological change through which we are passing. This fact creates challenges which must be dealt with by those involved in research, development, and teaching of mathematics. Researchers must attempt to identify some of the long-term fundamental questions that the future holds, and developers of mathematical techniques and tools must focus on the utility of the products of their efforts. Teachers perhaps face the heaviest of burdens, since they must identify future needs and educate our youth so as to be able to effectively meet them. In this lecture we discuss the problems of each of these communities from the point of view of present needs and future perceived challenges. Questions will be drawn from such diverse fields as materials processing in space and domestic heating and will, in turn, lead to remarks concerning mathematical curricula and industrial research efforts. The remarks made will be at least partly aimed at encouraging discussion and interchange among the listeners.

-Rescarch sponsored by Oak Ridge National Laboratory.

\subsection{2}

\section{THE INITIAL SPEED OF AN EMERGING PHASE CHANGE FRONT*}

\section{A. D. Solomon V. Alexindes}

\section{G. Wilson}

(Abstract of paper presented at Tel Aviv University, Tel Aviv, Israel, June 18,1984 )

Consider the melting of an initially totally solid slab $0 \leqslant X \leqslant L$ due to an imposed flux $q(t)$ at $x=0$. Let $f(x)$ be the initial temperature distribution on $[0, c]$, with $f(0)=$ melt 
temperature. Then the initial speed $X(0)$ of the emerging phase change front obeys

$$
\rho H X(0)=q(0)+K_{5} S^{\prime}(0)
$$

where $\rho, H, K_{s}$ are the density, latent heat and solid thermal conductivity of the material.

- Research sponsored by Applied Mathematical Sciences Reseaich Program. U.S. DOE Office of Energy Research.

\subsection{3}

\section{THE INITIAL VELOCTTY OF THE EMERGING FREE BOUNDARY IN A TWO-PHASE STEFAN PROBLEM WITH IMPOSED FLUX*}

\author{
A. Solomon V. Alexiades \\ D. G. Wilson
}

(Abstract of ORNL-6073, November 1984)

Consider the one-dimensional, two-phase Stefan Problem with an imposed flux in which the phase change front originates at the material boundary. We prove that, subject to suitable assumptions on the initial temperature and imposed flux, the initial speed of the phase change front is equal to the jump between the surface and initial fluxes at the boundary. Similarly, we prove that the frent $X(t)$ is continuously differentiable in the closed interval $\left[0, t^{\circ}\right]$ for some $i^{\circ}>0$.

- Research sponsored by Applied Mathematical Sciences Research Program, U.S. DOE Office of Energy Research.

\subsection{4}

\section{NUMERICAL SIMULATION OF A BINARY ALLOY SOLIDIFICATION PROCESS*}

\section{A. Solomon \\ V. Alexiades}

\section{C. Wilson}

[Ahs:ract of SIAM S. Siri. Sias. Compus. (in press)]

We describe the numerical implementation of a mathematical model of a binary alloy solidification process introduced in a previous paper. The model takes into account the "mushy" zone of dendritic growth between liquid and solid phase regions.

- Research sponsored by Applied Mathematical Sciences Research Program. U.S. DOE Office of Energy Research.

\subsection{5}

\section{MOVING BOUNDARY PROBLEMS IN PHASE CHANGE MODELS: CURRENT RESEARCH QUESTIONS*}

\author{
A. D. Solomon V. Alexiades \\ D. G. Wilson
}

[Abstract of Signum Newsletter 2e(2), 8 (1985)]

We present a brief picture of current research questions and activities on moving boundary problems.

- Research sponsored by Applied Mathematical Sciences Research Program, U.S. DOE Office of Energy Research.

\subsection{6}

\section{THE FORMULATION OF A HYPERBOLIC STEFAN PROBLEM*}
A. D. Solomon
V. Alexiades
D. G. Wilson
J. Drake

(Abstract of ORNL-6065, October 1984; also Quarterly of Applied Mathematics XI.Jl)(3), 295 (1985)]

The study of phase change processes governed by hyperbolic heat transfer is at an embryonic stage. We raise here some of the relevant questions and make some remarks on the formulation and qualitative behavior of a hyperbolic Stefan problem. In particular, we correct an error in the interface condition appearing in the literature and present an explicit solution to a simple one-phase problem and study its behavior. Finally we describe an enthalpy (weak) formulation for a two-phase problem and report on a number of numerical experiments based on it.

- Research sponsored by Applied Mathematical Sciences Research Program, U.S. DOE. Office of Finergy Research. 


\section{HEAT TRANSFER CONSIDERATIONS IN PASSIVE SOLAR WTTH ENERGY STORAGE*}

\author{
A. D. Solonon G. A. Geist
}

C. A. Sertia

(Abstract of paper presented at Ben-Gurion University. Beersheva, Israel. July 2-3. 1984)

Passive solar with energy storage presents us with a number of numerical and theoretical challenges. These arise from such factors as the high temperature differences between illuminated and non-illuminated surfaces, high gradients, and great sensitivity to such factors as the surface absorptivity. We discuss ways of overcoming these challenges and their physical bases.

-Research sponsored by the Thermal Energy Storage Program, U.S. DOE Office of Energy Research.

8.28

\section{HEAT TRANSFER AND PHASE CHANGE SIMULATION IN PCMSOL-L, A MODEL OF A DIRECT GAIN PASSIVE SOLAR STRUCTURE WITH LATENT HEAT STORAGE*}
A. D. Solomon
J. J. Tomitisos't
G. A. Geist
C. A. Serbin

(Abstract of peper presented at 1984 ASME/AICHE National Heat Transfer Conference, Niagara Falls. NY August 1984)

We have written a computer simulation model, PCMSOL-I, of a direct gain passive solar structure with latent heat storage. Some of the factors entering in its preparation are discussed. These include program structure, heat transfer relations, and outputs available.

\footnotetext{
- Research sponsored by the Thermal Energy Storage Program, U.S. DOE Office of Energy Research.

Engineering Technology Division.
}

\section{THE QUASI-STATIONARY APPROXIMATION FOR THE STEFAN PROBLEM WTTH A CONVECTIVE BOUNDARY CONDTTON*}

\author{
A. D. Solomon D. G. Witson
}

V. Alexiades

[Abstract of Intem J. Malh and Math Sciences 73) 549 (1984)]

We prove that the solution to the Stefan problem for a one-phase one-dimensional case converges to the quasi-stationary solution as the specific heat of the material tends to zero.

-Researci sponsored by Applied Mathematical Scieace Research Program, U.S. DOE Office of Enerty Research.

8.30

A DEVICE WHICH ALLOWS SPECIAL EVENT DETECTION IN CONTINUOUS SIMULATION LANGUAGES WTHHOUT DISCRETE BLOCKS*

\section{S. Thompsoen}

(Abstract of paper to be presented at the Summer Conference of the Society for Computer Simulation. Chicaso, IL, July 22-24, 1985)

Several simulation languages contain provisions for detecting and processing special events using discrete blocks. In this note, a device is described which also allows the user to detect and process special events using any similar continuous simulation language without the use of discrete blocks. The device is also useful for complex models for which it is not desirable or feasible to modify the model to incorporate the discrete blocks. The device has been included in the Babcock and Wilcox STACSL integrator which has been incorporated in the Advanced Continuous Simulation Language (ACSL). A well-known example is used to illustrate each of the two approaches using STACSL.

- Research sponsored by the Babcock and Wilcox Company and Oak Ridge National Laboratory. 
8.31

\section{EVENT DETECTION IN CONTINUOUS SIMULATION*}

\author{
S. Trompson P. G. Tutte'
}

(Abstract of paper presented at the International Conference for Power Plant Simulation. Cuernaraca, Morebos, Mexico. November 19-21. 1984)

Continuous simulation models often contain special events which must be detected and processed correctly in order to obtain a satisfactory solution. Event detection techniques may be incorporated into continuous simulation programs via root-finding methods. This paper first briefly reviews the previous use of root-finding in this context. It next describes the manner in which event detection techniques are incorporated into the well known ACSL continuous simulation program. Examples are then given to illustrate the use of these techniques.

-Research sponsored by the Babcock and Wilcox Company.

'Babcock and Wilcox Company, Lynchburg. VA

8.32

\section{TIME DEPENDENT LINEAR TRANSPORT III - CONVERGENCE OF THE DISCRETE ORDINATES METHOD*}

\section{G. Wilowa}

[Abstract of Transp. Theory Stat. Phys. 1214), 369 (1984)]

In this paper the uniform pointwise convergence of the discrete ordinates method for weak and strong solutions of the time dependent, linear transport equation posed in a multidimensional, rectangular parallelepiped with partially reflecting walls is established. The first result is that a sequence of discrete ordinates solutions converges uniformly on the quadrature points to a solution of the continuous problcm provided that the corresponding sequence of truncation errors for the solution of the continuous problem converges to zero in the same manner. The second result is that continuity of the solution with respect to the velocity variables guarantees that the truncation errors in the quadrature formula go to zero and hence that the discrete ordinates approximations converge to the solution of the continuous problem as the discrete ordinates become dense. An existence theorem for strong solutions of the continuous problem follows as a result.

- Research sponsored by Applied Matbematical Sciences Research Program, US. DOE Offree of Enerisy Research.

\subsection{3}

\section{VECTORIZATION OF MULTIDIMENSIONAL FINTTE DIFFERENCE SCHEMES WITH APPLICATION TO STEFAN-TYPE PROBLEMS*}

\author{
D. G. Wikson
}

(Abstract of paper to be presented at the Secood Society for Industrial and Applied Mathematics Conference on Paralkel Processing for Scienti.ic Computing. Noriolk, VA, November 20. 1985)

Vectorization tactics for multidimensional finite difference equations and the result of their application to an explicit scheme for an enthalpy formulation of three-dimensional Stefan-type problems are presented. The vectorization tactics consist of: (1) unfolding a three-dimensionat array of node points into one long vector, (2) factoring multidimensional calculations into several one-dimensional updates that can be performed simultaneously, and (3) using Boolean variables as multipliers in place of logical tests within loops. A special feature of Stefan-type problems, which is easily accommodated, is a discontinuous change of material properties as the phase change proceeds.

- Revearch sponsored by Applied Mathematical Sciences Research Program, U.S. DOE Office of Energy Research. 


\section{MATHEMATICAL MODELING - FUN AND GAMES WITH APPLICATIONS*}

\author{
D. G. Wison
}

(Abstract of paper presented at the University of Tennessee at Chattanooga. Chattanooga. TN, March 22 (985)

This is an elementary introduction to the application of mathematics. It will be emphasized that applied mathematics is interesting, enjoyable and useful. Ideas associated with formulating and analyzing mathematical models of physical processes will be discussed. A few example problems will be discussed beginning with balancing a checkbook and ending (hopefully) with a heat transfer problem involving a change of phase.

-Reacarch sponsored by lak Ridge Natioal Lebontory.

\subsection{5}

\section{MODELING BINARY ALLOY SOLIDIFICATION*}

\section{G. Wilson}

(Abstract of paper presented at the 21st Annual Meeting of the Society for Engineer ng Science, Blacksburg. VA, October 17. 1984)

A macroscopic mathematical model describing the idealized solidification of a mixture or binary alloy is presented. The formulation is global as a pair of conservation laws for mass and energy is valid over the entire region. This generalizes the idea of coupling the separate equations for the diffusion of temperature and material. The problem is formulated in a weak or distributional sense. It is not required to explicitly track the interfacial region which may develop into a mushy region. Both problem formulation and numerical results are discussed.

- Research sponsored by the Applied Mathematical Sciences kesearch Program, U.S. DOE Office of Energy Research.

\section{COMPUTER MODELING OF SOLIDIFICATION PROCESSE*}

\author{
D. G. Wilson
}

(Abstract of paper presented at Liniversity of Teanewee, Knoxville. TN. May 23. 1984; University of Minmi, Conl Gables. FL. April 5. 1985; Coacass Service Union AG. Zurich, Switzerland, May 14. 1985)

A survey is presented of the computer modeling techniques used to study various solidification processes in the Moving Boundary Problems research project at Oak Ridge National Laboratory. The processes under study include solidification of pure materials, binary alloy solidification, and very rapid melting and resolidification associated with laser annealing. The computer models represent a discrete, weak formulation of the corresponding mathematical models. Operationally what is implemented is an explicit finite difference scheme for something like the heat equation.

- Research sponsored by Applied Mathematical Sciences Research Program, U.S. DOE OTice of Energy Research.

\subsection{7}

\section{THE 1984 MODEL OF BINARY ALLOY SOLIDIFICATION*}

D. G. Wiken V. Akxiades

\author{
A. D. Solomon
}

(Abstract of paper presented at the International Colloquium on Free Boundary Problems, Maubuiscon France, June 7-15, 1984)

.. macroscopic mathematical model describing the idealized solidification of a mixture or binary alloy is presented. The formulation is globa: as a pair of conservation laws for mass and energy valid over the entire region. This generalizes the idea of coupling the separate equations for the diffusion of temperature and material. The problem is formulated in a weak or distributional sense. It is not required to explicitly track the interfacial region which may develop into a 
mushy region. Both problem formulation and numerical results are discussed.

- Research sponsored by Appliad Mathematial Sciences Research Program. U.S. LOE Office of Energy Research.

8.38

\section{A MODEL OF BINARY ALLOY SOLIDIFICATION*}

D. G. Wiken A. D. Solomon

V. Alexiades

[Abstract of Int. J. Num Meth Engr. 20, 1067 (1984); also ORNL/CSD-97. August 19821

A linear model for the solidification of a dilute binary alloy is presented. In this model the solidus and liquidus curves are linear. As a consequence, internal energy depends linearly upon temperature and concentration. The formulation is a generalization of the well-known enthalpy method to treat a phase change problem involving coupled heat and mass transfer. Both analytic and numerical formulations are given. Results from the lattet are presented and compared with an explicit solution of Rubinstein for a Stefan-like problem posed in a semi-infinite slab. Some remarks on the behavior of the explicit solution are given.

- Research sponsored by Appliod Mathematical Sciences Research Program, U.S. DOE Offoce of Energy Research.

\section{Computer Science}

8.39

\section{AN ALGORITHM TO COMPUTE A SPARSE BASIS OF THE NULL SPACE*}
M. W. Berry'
M. T. Heath
I. Kasetzot
M. Lawó
R. J. Plemmons ${ }^{\dagger}$
R. C. Ward

[Abstract of Numerische Mathemasik (in press)]

Let $A$ be a real $m \times n$ matrix with full row rank $m$. In many algorithms in engineering and science, such as the force method in structural analysis, the dual variable method for the Navier-Stokes equations or more generally null space methods in quadratic programming, it is necessary to compute a basis matrix $B$ for the null space of $A$. Here $B$ is $n \times r, r=$ $n-m$, of rank $r$, with $A B=0$. In many instances $A$ is large and sparse and often banded. The purpose of this paper is to describe and test a variation of a method originally suggested by Topeu and called the turnback algorithm for computing a banded basis matrix $B$. Two implementations of the algorithm are given, one using
Gaussian elimination and the other using orthogonal factorizeion by Givens rotations. The FORTRAN softwar: was executed on an IBM 308 I mainframe computer with an FPS-164 attached array processor at the Triangle Universities Computing Center near Raleigh, NC. Test results on a variety of structural analysis problems, including two and three-dimensional frames, plane stress, plate bending, and mixed finite element problems, are discussed. These results indicate that both implementations of the algorithm yielded a well-conditioned, banded, basis matrix $B$ when $A$ is well conditioned. However, the orthogonal implementation yielded a better conditioned $B$ for large, ill-conditioned problems.

\footnotetext{
-Research sponsored by the Applied Mathematical Sciences Research Program. U.S. DOE Office of Energy Research; by U.S. Air Force; and by National Science Foundation.

'Departments of Mathematics and Computer Science. North Carolina State University, Raleigh, NC.

tDepartment of Commerce. Hitotsubashi University, Kunitachi, Tokyo 186. Japan.

Department of Civil Engineering. Universitat Gesamtho chischule, Essen Postfach 103764, 4300 Essen I, West Germany.
} 
8.40

\section{ORTHOGONAL SCHEMES FOR STRUCTURAL OPTTMIZATION*}

\author{
M. W. Berry ${ }^{\prime}$ \\ M. T. Heath \\ R. J. Plemenos' \\ R. C. Ward
}

[Abstract of Trans. First Army Conference on Applied Markemarics and Compuring, p. 477, U.S. Army Research Offroe Report 84-1 (1984)]

Historically there are two principal methods of matrix structural analysis, the displacement (or stiffness) method and the force (or flexibility) method. In recent times the force method has been used relatively little because the displacement method has been deemed easier to implement on digital umputers, especially for large sparse systems. The force method has theoretical advantages, however, for multiple redesign problems or nonlinear elastic analysis because it allows the solution of modified problens without restarting the computation from the beginning. In this paper we give an implementation of the first phase of the force method which is numerically stable and preserves sparsity. A primary feature of our work is the development of an efficient algorithm for computing a banded basis for the null space by orthogonal decomposition. Numerical test comparisons for several practical structural analysis problems are provided.

- Research sponisred by Applied Mathematical Sciences Research Program. U.S. DOE Office of Energy Research.

'Department of Mathematica and Computer Science, North Carolina State University, Raleigh, NC.

8.41

\section{PROGRESS REPORT ON JAPANESE COMPUTER PROJECTS*}

\section{K. O. Bowman}

[Abstract of Scientific Bulletin I0, 91 (1985)]

This report discusses Japan's current research on logical computers (also identified as artificial intelligence research) and also the development work now being performed on parallel processors.
Finally it describes how supercomputers (vector computers). which are already being manufactured commercially in Japan, are being put to use and developed further.

- Recearch sponsored by Office of Naval Research, Far Easa Ciñce.

\subsection{2}

\section{THE HYPERCUBE ARCHTTECTURE AND SOME ASPECTS OF DATA FLOW FOR PARALLEL COMPUTATION OF MISSIZED MATRIX PROBLEMS*}
R. E. Fandertic
G. A. Geist

(Abstract of paper presented at the Parallel Compoting Seminar. Matberastios Depertment. North Carolina State University, Rakeigh. NC. April 8-12, 1985)

The Cholesky algorithm will be used as an example on a torus array of processors for discussing such properties as optimal number of time steps, speedup, processor utilization and synchronization. An $n$-dimensional hypercube is a computer with 2 to the $n$ processors, each processor being capable of passing a message directly to $n$ processors and indirectly to the others. Global organization and programming considerations will be given for a hypercube architecture. Indications will be given as to how to embed or simulate other arrays of processors in a hypercube.

- Rescarch sponsored by Applied Mathematical Sciences Research Program, U.S. DOE Office of Energy Research.

\subsection{3}

\section{TORUS DATA FLOW FOR PARALLEL COMPUTATION OF MISSIZED MATRIX PROBLEMS*}
R. E. Fundertic
G. A. Geiat

(Abatract of ORNL-6125, February 1985; also Limear Alsebra and Its Applications (in press); aleo paper presented at the Society for Industrial and Applied Mathematica Conference on Applied Linear Algebra, Raleigh, NC, April 29. May 2. 1985]

Large dense systems of equations can be solved efficiently on a processor array with only 
nearest neighbor communication using a dataf. w approach. Although this approach requires no global synchronizaiion, the development of our simulator provided additional understancing of the necessity of local synchronization of data movement when there are more computational nodes than processors (missized). New definitions for missized problems and parallel algorithm efficiency are given. For the Cholesky decomposition the lower triangle was mapped onto a torus configuration of processors. This implementation is compared to the thecretical peak speed. Various time step, efficiency, and processor utilization plots are given.

- Research sponsored by the Applied Mathematical Sciences Research Program. U.S. DOE Office of Energy Research.

\subsection{4}

\section{SENSITIVITY OF THE STATIONARY DISTRIBUTION VECTOR FOR AN EFGODIC MARKOV CHAIN*}
R. E. Funderlic
C. D. Meyer, Jr. ${ }^{\dagger}$

(Abstract of ORNL-6098 (January 1985); a'so Linear Alge. bra and lts. Applications (in press); also paper presented al the Society or Industrial and Applied Mathematics Confer ence on Afplied Linear Algebra, Raleigh. NC, April 29 May 2, 198 il

Stationary distribution vectors $p^{\infty}$ for Markov chains with associated transition matrices $T$ are important in the analysis of many models in the mathematical sciences, such as queuing networks, input-outp'st economic models, and compartmenta! tracer analysis models. The purpose of this paper is to provide insight into the sensitivity of $p^{x}$ to perturbations in the transition probabilities of $\dot{i}$ and to understand some of the difficulties in computing an accurate $p^{\infty}$. The group inverse $A^{\prime \prime}$ of $I-T$ is shown to be of fundanuental importance in understanding sensitivity or conditioning of $p^{x}$. The main result shows that if there, is a state that is accessible from every other state and the corresponding column of $T$ has no small offdiagonal elements, then $p^{\infty}$ cannot he sensitive to small perturbations in $T$. Ecological examples are given. A new stable algorithm for calculating $A^{\prime \prime}$ is described.
- Research sponsored by Applied Mathematical Sciences Research Program, U.S. DOE OfTice of Encrgy Research.

'Mathematics Department. North Carolina State University, Raleigh, NC.

8.45

\section{A COMBINED DIRECT-ITERATIVE METHOD FOR CERTAIN M-MATRIX LINEAR SYSTEMS*}

\author{
R. E. Funderlic $\quad$ R. J. Plemmons ${ }^{\dagger}$
}

[Abst:act of SIAM J. Alg. Disc. Meth. S(1), 33 (1984); also OKNL.CSD-112, [983]

Large, sparse, irreducible singular (column diagonal dominant) $M$-matrices $A$ occur in various applications including queueing networks, input-output analysis, and compartmental analysis. Our splitting $A=M-N$ with the matrix $M$ having symmetric zero structure is a regular splitting, and these split ings induce a combined direct-iterative solution :o $A x=0$. A sparse LU factorization of a symmetric permutation of $A$ can be obtained using a standard symmetric ordcring scheme such as minimum degree. No pivoting for stability is necessary. Splitting $s^{t}$ raiegies based on a tolerance factor are also discussed and some numerical experience is given.

-Research sponsored by Applied Mathematical Sciences Research Program. U.S. DOE Office of Energy Research.

${ }^{t}$ Department of Mathematics and Computer Science, North Carolina State University, Raleigh, NC.

8.46

\section{UPDATING LU FACTORIZATIONS FOR COMPUTING STATIONARY DISTRIBUTIONS*}

\section{R. E. Funderlic R. J. Plemmons ${ }^{t}$}

(Abstract of paper presented at the Society for Indus/ rial and Applied Mathematics National Meeting. University of Washington, Seattle, WA, July 16 20, 1984; also presented at the Gatlinburg 9 International Conference on Numerical Algebra, Waterloo, Ontario Canada, July (1, 1984)

The computation if stationary probability dis. tributions for Markov chains is important in the 
analysis of many models in the mathematical sciences, such as queueing network models, inputoutput economic models, and compartmental tracer analysis models. These computations often involve the solution of large-scale homogeneous linear equations by Gaussian elimination, where $A$ is a Q-matrix; i.e., $A=\left(a_{i j}\right)$ is irreduc ble, $a_{i j} \leqslant 0$ for all $i \neq j$ and has zero column sums. The stationary distibutions are the components of the unique solution vector $x$ of positive components whose sum is one. Stable direct methods for computing $x$ by triangular factorization $A=L U$ have received considerable aitention recently, and the purpose of this talk is to provide a stable method for updating the factor; $!$. and $U$ in $O\left(N^{2}\right)$ flops in :he case where a column of $A$ is modified. Rather than update $L$ and $U$, the ShermanMorrison formula can be used if only one column is modified. Transition rate changes in queueing networks cause several columns to change. Updating formulas are derived here using an approach similar to that for updating the Cholesky factor of a symmetric positive definite matrix after the addition of a rank one matrix. T'c algorithm is effective more generally for any matrix that has a stable $L U$ factorization and for which the updated matrix has a stable $L U$ factorization. An error analysis for the $L U$ update algorithm is outlined along the lines of that given for the Cholesky update by Fletcher and Powell. Modifications of the algorithm based on the error analysis and other considerations will be given.

- Research sponsored by Applied Mathematical Sciences Researci Program, U.S. DOE Office of Energy Research.

'Department of Mathematics an Computer Science, North Carolina State University, Raleigh. NC.

\subsection{7}

\section{FORTRAN PROGRAMMING ON AN IBM PC*}

\section{P. W. Gaffney}

(Abstract of paper presented at Numerical Computation and Mathematical Software for Microcomputers Conference, Boulder. (O, March 1921,1984$)$

The purpose of this talk is to answer the question: is it feasible to program an IBM personal computer in FORTRAN to do scientific computations?

In particular, the scientific computations that are addressed in this question are those that would normally uccur in a research and development establishment. In such a situation it is usual to do FORTRAN programming using a compiler rather than an interpreter. Consequently, the talk reviews the available compilers suitable for the IBM PC. By addressing the elementary issues that are felt to be relevant in such a review (for example, whether the compiler works, and what features from the FORTRAN language are present or missing in the compiler), it is possible to eliminate many of the existing compilers. One of the remaining compilers that may be suitable for the above envirenment is the new Microsoft FORTRAN compiler.

This talk gives the results of a detailed analysis of the properties and performance of this compiler when it is applied to typical good quality software. The particular features that make the Microsoft compiler attractive, in a scientific context, are identified and discussed. Furthermore, it is shown how to use the facilities of the IBM I'C's operating system in order to simplify the process of programming this micricomputer.

The important iss'te of creating object module libraries is discussed with a view to facilitating the use of packages such as EISPACK and LINPACK. The experiences gained in using some of this software on the IBM PC, together with the new NAG PC50 collection of codes, will be provided at the end of the talk.

- Research sponsored by the Computing and Telecommunications Division, Martin Marietta Energy Systems, Inc. 
8.48

\section{SOME ASPECTS OF A DATA FLOW ALGORTHM FOR CHOLESKY DECOMPOSITION*}

\section{G. A. Geist R. E. Funderlic \\ J. Meyering}

(Abstract of paper presented at DOE Numerical Analysis Special Interest Gr Jup Meeting. Argonne National Laboratory. Argonne. IL. September 18-19. 1984)

Solving dense systems of equations on an array processor with only nearest neighbor comreunication requires careful algorithm planning to produce peak performance. When the order of the matrix is large relative to the square root of the number of processors, the problem is said to be missized. Data flow algorithms for missized problems often require local synchronization schemes in addition to the flow of data to stably execute an algorithm. In this talk we discuss some experiences we have had with synchronizing a data flow algorithm for the Cholesky decomposition of missized problems.

-Research sponsored by Applied Mathematical Sciences Research Drogram, U.S. DOE Office of Energy Research.

\subsection{9}

\section{PARALLEL CHOLESKY FACTORIZATION ON A HYPERCUBE MULTIPROCESSOR*}

\author{
G. A. Geist M. T. Heath
}

(Abstract of ORNL-6190, August 1985)

Two types of message-passing parallel algorithms are developed for solving symmetric systems of linear equations on a hypercube multiprocessor. One type involves broadcast communication among processors, and the other involv $s$ communication along a ring of processo. Details are provided in the form of $C$ prograr.. that implement the algorithms on a hypercube simulator and which should run with little moditication on real hypercube hardware. Performance of the various algorithrns is demonstrated by means of processor utilization graphs and parallel speedup curves.

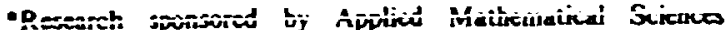
Research Program, U.S. DOE Office of Energy Research.

\subsection{0}

\section{PARALLEL CHOLESKY FACTORIZATION ON A MULTIPROCESSOR*}

\author{
A. George ${ }^{\dagger}$ M. T. Heath
}

\section{J. $\operatorname{Lin}^{t}$}

[Abstract of ORNL-6124. March 1985; also Linear Algebra and Its Applications (ith press)]

A parallel algorithm is developed for Cholesky factorization on a multiprocessor. The algorithm is based on self-scheduling of a pool of tasks. The subtasks in several variants of the basic elimination algorithm are analyzed for potential concurrency in terms of precedence relations, work profiles, and processor utilization. This analysis is supported by simulation results. The most promising variant, which we call columnCholesky, is identified and implemented for the Denelcor HEP multiprocessor. Experimental results are given for this machine.

\footnotetext{
- Research sponsored by Canadian Natural Sciences and Engineering Research Council; by Applied Mathematical Sciences Research Program, U.S. DOE Office of Energy Research; and by U.S. Air Force, Office of Scientific Research.

'Department of Computer Science. University of Waterloo, Waterloo. Ontario, Canada.

IDepartment of Computer Science, York University, Downsview, Ontario, Canada.
}

\subsection{1}

\section{SOLUTION OF SPARSE UNDERDETERMINED SYSTEMS OF LINEAR EQUATIONS*}
A. George ${ }^{+}$
M. T. Heath
E. Ng

[Abstract of SIAM J. Sici. Siat. Comput. S/4h 988 (1984); also Society for Industrial and Applied Mathematics Nationai Mecting. University of Washington. Seattle, WA, Suly $16 \cdot 20$, 1984: also ORNL/CSD-121, 1983]

The problem of computing the minimumnorm solution to a sparse, underdetermined system of linear equations is considered. An algorithm is developed which is based on orthogonal 
factorization. The algorithm is generalized to cope with systems which are rank deficient, inconsistent, or require column updating.

* Research spoosored by Applied Mathemntical Sciences Program, U.S. DOE OfTice of Energy Researta, and by Canadian Natural Sciences and Engincering Research Council.

'Department of Computer Science, University of Watertoo, Waterloo, Ontario, Canada.

\subsection{2}

\section{HOUSEHOLDER REFLECTIONS VERSUS GIVENS ROTATIONS IN SPARSE ORTHOGONAL DECOMPOSITION*}

\section{A. Georget J. Lid}

(Abstract of Technical Report, Computer Science Department. University of Waterloo, Nowember 1984)

It has been generally assumed that the use of Givens rotations provides significant advantages over the use of Householder transformations for the orthogonal decomposition of sparse matrices. It is also generally acknowledged that the opposite is true for dense matrices. In tinis paper, a way of applying Householder refloctions is described which is competitive or superior to the use of Givens rotations for sparse orthogonal deco:aposition. In other words, the advantage of Hcuseholder over Givens for dense matrices can carry over to the sparse case, provided that the implementation of the Householder scheme is done in a certain way. The approach relies heavily on the idea of general row merge trees developed hy Liu. Results of numerical experiments are provided which demonstrate the advantages of the new scheme. The method also appears to be attractive for use on vector computers.

-Resurch aponsored by U.S. Air Force, Orfice of Scientific Research and by Canadian Natural Sciences and Engineering Research Council.

'Department of Computer Science, University of Waterloo, Waterloo, Ontario, Canada.

tDepertment of Computer Science, York University, Downsview, Ontario, Canada.
ORTHOGONAL REDUCTION OF SPARSE MATRICES TO UPPER TRIANGULAR FORM USING HOUSEHOLDER TRANSFORMATIONS*
A. George
E. Ng

[Abseract of SIAM J. Sci Siar. Comput. (in preas), also Technical Report CS-84-05, Computer Science Department University of Waterioo, 1984]

In this paper we consider the problem of predicting the fill-in that occurs in the $Q R$ decomposition of sparse matrices using Householder transformations. We show that a static data structure can be used throughout the numerical computation, and that the Houscholder transformations can be saved explicitly in a compact format.

\footnotetext{
- Research sponsored by U.S. Air Force, Office of Scientific Research and by Canadian Natural Sciences and Engineering Research Council.

Department of Computer Science, University of Waterbo, Watertoo, Ontario, Canada.
}

\subsection{4}

\section{SYMBOLIC FACTORIZATION FOR SPARSE GAUSSIAN ELIMINATION WITH PARTIAL PIVOTING*}
A. George ${ }^{\prime}$
E. $\mathbf{N g}$

(Technical Report CS-84-43, Computer Science Department, University of Waterloo, 1984)

Let $A x=b$ be a large sparse nonsingular sys$\mathrm{em}$ of linear equations to be solved using Gaussian elimination with partial pivoting. The factorization obtained can be expressed in the form $A=P_{1} M_{1} P_{2} M_{2} \ldots P_{n-1} M_{n-1} C^{\prime}$, where $P_{k}$ is an elementary permutation matrix reflecting the row interchange that occurs at step $k$ during the factorization, $M_{k}$ is a unit lower triangular matrix whose $k$-th column contains the multipliers, and $U$ is an upper triangular matrix. 
Consider the $k$-th step of the elimination. Suppose we replace the structure of row $k$ of the parially reduced matrix by the union of the structures of those rows which are candioates for the pivot row and then perform symbolically Gaussian elimination without partial pivoting. Assume that this is done at each step $k$, and let $\bar{L}$ and $\bar{U}$ denote the resulting lower and upper triangular matrices respectively. Then the structures of $\bar{L}$ and $\bar{U}$ respectively contain the struc$n-1$

tures of $\sum_{k=1}^{n} M_{k}$ and $U$. This paper describes an algorithm which determines the structure of $\bar{L}$ and $\bar{U}$ and sets up an efficient data structure for them. Since the algorithm depends only on the structure of $A$, the data structure can be created in advance of the actual numerical computation, which can then be performed very efficiently using the fixed storage scheme. Although the data structure is more generous than it needs to be for any specific sequence $P_{1}, P_{2}, \ldots, P_{n-1}$, experiments indicate that the approach is competitive or superior to conventional methods. Another important point is that the storage scheme is large enough to accommodate the $\mathrm{QR}$ factorization of $A$, so it is also useful in the context of computing a sparse orthogonal decomposition of $A$. The algorithm is shown to execute in time bounded by $|\bar{L}|+|\bar{U}|$, where $|M|$ denotes the number of nonzeros in the matrix $M$.

- Research sponsored by U.S. Air Force, Office of Scientific Rescarch and by Canadian Nalural Sciences and Engineering Research Council.

'Department of Computer Science. University of Waterloo. Waterloo, Ontario, Canada.

\subsection{5}

\section{AN IMPLEMENTATION OF GAUSSIAN ELIMINATION WITH PARTIAL PIVOTING FOR SPARSE SYSTEMS*}
A. George'
E. Ng

[Ahstract of SIAM J. Sri. Stat. Comput. $6(2), 390$ (1985)]

In this paper, we consider the problem of solving a sparse nonsingular syst $=m$ of linear equations. We show that the structures of the triangular matrices obtained in the LUdecomposition of a sparse nonsingular matrix $A$ using Gaussian elimination with partial pivoting are contained in those of the Cholesky factors of $A^{T} A$, provided that the diagonal elements of $A$ are nonzero. Based on this result, a method for solving sparse linear systems is then described. The main advantage of this method is that the numerical computation can be carried out using a static data structure. Numerical experiments comparing this method with other implementations of Gaussian elimination for solving sparse linear systems are presented, and the results indicate that the method proposed in this paper is quite competitive with other approaches.

\footnotetext{
- Research sponsored by Applied Mathematical Sciences Research Program, U.S. DOE Office of Energy Research. and by Canadian Natural Sciences and Engineering Research Council.

'Department of Computer Science, University of Wrte.!oo. Waterloo, Ontario, Canada.
}

\subsection{6}

\section{PARALLEL CHOLESKY FACTORIZATION IN MESSAGE-PASSING MULTIPROCESSOR ENVIRONMENTS*}

\section{T. Heath}

(Abstract of ORNL-6150, May 1985)

Parallel algorithms are presented for computing the Cholesky factorization on multiprocessurs having only private local memory. Synchronization of multiple processes is based on message passing. Several possible processor interconnection networks are considered.

- Revearch sponsored by Applied Mathematical Sciences Research Program, U.S. DOE Office of Energy Resenrch. 
8.57

\section{NUMERICAL METHODS FOR LARGE SPARSE LINEAR LEAST SOUARES PROBLEMS*}

\section{T. Heath}

[Abstract of SIAM J. Sci. Stat. Comput. $5(3) 497$ (1984)]

Large sparse least squares problems arise in many applications, including geodetic network adjustments and finite element structural analysis. Although geodesists and engineers have been solving such problems for years, it is only relatively recently that numerical analysts have turned attention to them. In this paper we present a survey of numerical methods for large sparse linear least squares problems, focusing mainly on developments since the last comprehensive surveys of the subject published in 1976. We consider direct methods based on elimination and on orthogonalization, as well as various iterative methods. The ramifications of rank deficiency, constraints, and updating are also discussed.

- Research sponsored by National Geodetic Survey of the National Ocean Survey. NOAA, U.S. Department of Commerce; and by Applied Mathematical Sciences Researcis Program, U.S. DOE Ortice of Energy Research.

\subsection{8}

\section{SOME SPARSE MATRIX PROBLEMS IN STRUCTURAL ANALYSIS*}

\section{T. Heath}

(Abstract of paper presented at the Society for Industrial and Applied Mathematica Conference on Applied Linear Algebra, Raleigh, NC, April 29 May 2, 1985)

The well-known stiffness method of finite element structural analysis leads to the well-studied problems of solving overdetermined and sym. metric positive definite systems of linear equations. The lesser-known flexibility (or force) method leads to the less well-studied, but equally interesting, problem of solving underdetermined linear systems and the related problems of computing a basis matrix for the null space of the equilibrium matrix and solving generalized least squares problems. In this talk, we review recent work on solving these problems for large sparse matrices.

- Research sponsored by Applied Mathematical Science. Research Program, U.S. DOE Office of Energy Research.

8.59

\section{SPARSE MA TRIX COMPUTATIONS*}

\section{T. Heath}

[Abstract of paper presented at the 23rd IEEE Conference on Decision and Control, Las Vegas, NV, December 12-14, 1984; Proc. Vol. 2, 662 (1984)]

Numerical linear algebra plays a vital role in all parts of computational mathematics, such as differential equatione and optimization, and in many application areas, such as control theory. Large systems in these areas generally lead to linear algebra problems involving large sparse matrices (i.e., matrices of large dimension, but whose entries are mostly zeros). Efficient use of computer resources in solving such sparse matrix problems requires special techniques which have a distinct flavor compared to those used for dense matrices. We survey the current state of the ant in sparse matrix computations, including the solution of systems of linear algebraic equations, linear least squares problems, and eigenvalue problems. Emphasis is placed on direct methods, but iterative methods are also considered. We also emphasize the concrete and useful expression of algorithms in the form of computer software.

- Research sponsored by Applied Mathematical Sciences Research Program, U.S. DOE Office of Energy Research. 
8.60

\section{COMPUTING THE SINGULAR VALUE DECOMPOSITION OF A PRODUCT OF TWO MATRICES*}
M. T. Heath
A. J. Lamb'
C. C. Paiget
R. C. Ward

[Abstract of ORNL-6118, January 1985; dse SIAM J. SCi. Star. Comput. (in press)]

An algorithm is developed for computing the singular value decomposition of a product of two general matric's without explicitly forming the product. The algorithm is based on an earlier Jacobi-like method due to Kogbetliantz anć uses plane rotations applied to the two matrices separately. A trianguiar variant of the basic algorithm is developed that reduces the amount of work required.

\footnotetext{
-Research supported by Applied Mathematical Sciences Research Program, U.S. DOE Office of Energy Research; by U.S. Air Force Office of Scientific Research; by National Science Foundation, and by Canadian Natural Sciences and Engineering Research Council.

tDepartment of Electrical and Computer Engineering. University of California, Santa Barbara, CA.

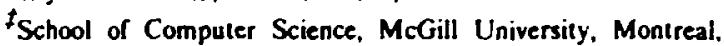
Canada.
}

8.61

\section{A PIPELINED GIVENS METHOD FOR COMPUTING THE QR FACTORIZATION OF A SPARSE MATRIX*}

\section{T. Heath $\quad$ D. C. Sorensen ${ }^{\prime}$}

[Abstract of Linear Algebra and Irs Applicarions (in press)]

This paper discusses an extension of the Pipelined Givens method for computing the $Q R$ factorization of a real $m \times n$ matrix to the case in which the matrix is sparse. When restricted to one process, the algorithm performs the same computation as the serial sparse Givens algorithm of George and Heat.1. Our implementation is compatible with the data structures used in SPAKSPAK. The pipelined algorithm is well suited to parallel computers having globally shared memory and low overhead synchronization primitives, such as the Denelcor HEP, for which computational results are presented. We point out certain synchronization problems that arise in the adaptation to the sparse setting and discuss the effect on parallel speedup of accessing a serial data file.

-Research sponsored by Applied Mathematical Sciences Research Program, U.S. DOE Office of Energy Research.

'Argonne National Laboratory. Argonne, IL.

\subsection{2}

\section{SPARSE ORTHOGONAL SCHEMES FOR STRUCTURAL OPTIMIZATION USING THE FORCE METHOD*}

\section{T. Heath R. C. Wo:d \\ R. J. Plemmons ${ }^{t}$}

[Abstract of SIAM J. Sci. Stat. Comput. 5(3), S14 (1984); also ORNL/CSD-119, May 1983]

Historically there are two principal methods of matrix structural analysis, the displacement (or stiffness) method and the force (or Mexibility) method. In recent times the force method has been used relatively little because the displacernent method has been deemed easier to implement on digital computers, especially for large sparse systems. The force method has theoretical advantages, however, for multiple redesign problems or nonlinear elastic analysis because it allows the solution of modified problems without restarting the computation from the beginning. In this paper we give an implementation of the force method which is numerically stable and preserves sparsity. Although it is motivated by earlier elimination schemes, in our approach each of the two main phases of the force method is carried out using orthogonal factorization techniques recently developed for linear least squares problems.

-Research sponsored hy Applied Mathematical Sciences, U.S DOE Office of Energy Research.

'Department of Mathematics and Computer Science, North Carolina State Iniversity, Raleigh, NC. 


\section{ON GENERAL ROW MERGING SCHEMES FOR SPARSE GIVENS TRANSFORMATIONS*}

\author{
J. W. H. Liv' ${ }^{\dagger}$
}

(Abstract of Technical Report CS-83-04, Computer Science Department, York University, November 1984)

This paper introduces general row merging schemes for the QR-decomposition of sparse matrices by Givens rotations. They can be viewed as a generalization of row rotations to submatrix rotatiors (or merging) in the recent method by George and Heath. Based on the column ordering and the structure of the given sparse matrix, we present an algorithm to determine automatically a sequence of submatrix rotations appropriate for sparse decomposition. It is shown that the actual numerical computation can be organized as a sequence of reductions of two upper trapezoidal full submatrices into another upper trapezoidal full matrix. Experimental results are provided to compare the practical performance of the proposed method and the George-Heath scheme. Significant reduction in arithmetic operations and factorization time is achieved in exchange for a very modest increase in working storage. The interpretation of general row merging as a special variable row pivoting method is also presented.

- Research sponsored by U.S. Air Force, OPrice of Scientific Research, and by Canadian Natural Sciences and Engineering Research Council.

tuepartment of Computer Scie lce, York University. Downsview, Ontario, Canada.

\section{PARALLEL BLOCK JACOBI EIGENVALUE ALGORTTHMS USING SYSTOLIC ARRAYS*}

\section{S. Scott ${ }^{\dagger}$ M. T. Heath}

\section{R. C. Ward}

[Abstract of Technical Report CS-85-01, Computer Science Department, University of Texas, January 1985; also Limear Algebra and Its Applications (in press)]

Systolic arrays have become established in principle, if not yet in practice, as a wdy of increasing computational speed for problems in linear algebra, such as computing eigenvalues, by exploiting low-level parallelism. Any systolic device that is actually implemented in silicon will necessarily have an upper limit on the size of the problems that can be solved. In this paper we consider the questions of whether such a systolic device for symmetric eigenvalue problems can be used to solve larger problems and, if so, whether such use would place any constraints on the design of the underlying systolic array. We will see that the answer to both questions is "yes," although the restrictions on the systolic array are mild. Our approach is to partition a iarge matrix into blocks that are smali cnough to be processed by the systolic device, and a Jacobi-iike process is then carried out at the block level. If multiple copies of the systolic device are available, then this block Jacobi process can itself be implemented with a higher-level parallelism. To make any progress it is necessary to reurder the matrix between calls to the systolic arrays.

- Rescarch sponsored by Applied Mathematical Sciences Research Program. U.S. DOE Office of Energy Research, and by U.S. Air Force Orfice of Scientific Resentch.

'Computer Science Department, University of Texas, Austin, TX. 


\section{Statistics}

\section{MISCLASSIFICATION PROBABILITTES FOR SECONDORDER DISCRIMINANT FUNCTIONS USED TO CLASSIFY BIVARIATE NORMAL POPULATIONS*}

\author{
C. K. Baye' J. J. Beauchamp \\ V. E. Kanet
}

[Abstract of Communications in Slatistics B13. 669 (1984)]

Consider the two population bivariate normal discrimination problem where $X$ is observed from population $\mathrm{II}_{Y}$ with $Y=0$ or 1 . The probability density function for $\Pi_{Y}$ is $N\left(U_{Y}, \Sigma_{Y}\right)$ where $U^{\prime} Y$ $=\left(U_{Y 1}, U_{Y_{2}}\right)$ is the mean vector and $\Sigma_{Y}$ is the 2 $\times 2$ ccvariance matrix constructed from the variances $\operatorname{Var}\left(X_{Y_{1}}\right)=S_{Y 1}^{2}$ and $\operatorname{Var}\left(X_{Y_{2}}\right)=S_{Y_{2}}^{2}$ and the correlation $\rho_{Y}$. The prior probability for $\Pi_{Y}$ is $\pi_{Y}$ where $\pi_{0}+\pi_{1}=1$. A classification rule $Q(X)$ assigns an arbitrary $X$ to $\Pi_{0}$ if $Q(X)<$ 0 and to $\Pi_{1}$ if $Q(X)>0$ where

$$
\begin{aligned}
Q(\mathbf{X})= & B_{0}+B_{1} X_{1}+B_{2} X_{2} \\
& +B_{3} X_{1} X_{2}+B_{4} X_{1}^{2}+B_{3} X_{2}^{2} .
\end{aligned}
$$

For any application, the $B$ 's may take on any real values and one or more of the B's can be equal to zero for a particula: discriminant function. Subroutine QDFERR calculdtes the misclassification probabilites for $Q(X)$ defined by

$$
\begin{aligned}
& \rho_{0}=\operatorname{Pr}[Q(X)>0 \mid Y=0], \\
& p_{1}=\operatorname{Pr}[Q(X)<0 \mid Y=1], \text { and } \\
& p_{T}=\pi_{\infty} p_{0}+\pi_{1} p_{1} .
\end{aligned}
$$

- Research sporisored by Applied Mathematical Sciences Research Program, U.S. DOE Office of Energy Reses rch.

'Oemputing and Telecommunications Division.

tFoid Motor Company. Dearborn. MI.

\section{APPLICATION OF THE POWER-SHIFT TRANSFORMATION*}

\author{
J. J. Beauchamp V.E. Kane'
}

[Abstrzx of J. Statist. Comput. Simul. B19, 35 (1984)]

Some of the practical consequences of entertaining the power-shift transformation are examined for bcth analysis of variance and simple random sampling data. The appropriateness of different procedures for estimating the transformation parameters is also considered. Standard maximum likelihood $(\mathrm{ml})$ estimation is shown not to be feasible for the joint estimation of the power-shift parameters. However, it is possible to compute local $\mathrm{ml}$ estimates for sunie problems, but special estimation methods may be necessary. An alternative weighted order statistic (wos) estimator is shown to be useful in some situations. Characteristics of $\mathrm{ml}$ and wos estimation for the power-shift transformation are examined by a Monte Carlo experiment. A Box and Cox (1964) example and several other data sets are used to illustrate the estimation procedures.

- Rescarch sponsored by Applied Mathematical Sciences Research Program, U.S. DOE Orfice of Energy Research.

'Ford Moior Company, Dearborn, MI.

8.67

\section{SURSAMPLING PROGRAM FOR THE ESTIMATION OF FISH IMPINGEMENT*}

\section{J. J. Beauchump K. D. Kumar \\ [Abstrart of Environ. Manage. 8, 339 (1984)]}

Federal regulations require operators of nuclear and coal-fired power-generating stations to estimate the number of fish impinged on intake screens. During winter months, impingement 
may range into the hundreds of thousands for certain species, making it impossible to count all intake screens completely. We present graphs for determining the appropriate "optimal" subsample that must be obtained to estimate the total number impinged. Since the number of fish impinged tends to change drastically within a short time period, the st'hsample size is determined based on the most receat data. This allows for the changing nature of the species-age composition of the impinged fish. These graphs can also be used for subsampling fish catches in an aquatic system when the size of the catch is too large to sample completely.

- Research sponsored by U.S. DOE Office of Health and Environmental Research.

'SAS Institule, Cary, NC.

\subsection{8}

\section{EXTENDED MOMENT SERIES AND THE PARAMETERS OF THE NEGATIVE BINOMIAL DISTRIBUTION*}

K. O. Bowman

[Abstract of Biometrics 40, 249 (1984)]

For the negative binomial distribution with mean $\mu$ and variance $\mu-\mu^{2} / k$, the simplest moment estimator of $k$ is $k^{*}=\bar{x}^{2} /\left(m_{2}-\bar{x}\right)$, where $m_{2}$ is the second central sample moment. Using a computer-extended Taylor series, studies are made of the mean and variance of $k^{*}$, the series being taken as far as the coefficient of $n^{-12}, n$ being the sample size. A summatory algorithm is needed for the divergent series for the moments.

- Research sponsorod by Applied Mathematical Sciences Research Program, U.S. DOE Ofrice of Energy Research.
8.69

\section{COMPUTATJON OF THE POLYGAMMA FUNCTIONS*}

\section{K. O. Bowman}

[Abstract of J. Communcations in Statistics 13(3), 409 (1984)]

An algorithm is presented for computing the psi(digamma) and other polygamma functions.

The algorithm computes

$$
\begin{gathered}
\psi(x)=d\left(\ln \Gamma(x)^{y} / d x=\Gamma^{\prime}(x) / \Gamma(x),\right. \\
\psi_{s}(x)=d^{s} \psi(x) / d x^{s},
\end{gathered}
$$

for real positive values of $x$ between 0.01 and 4000 and $s$ up to 20.

The computed values of $\psi(x)$ are checked extensively against published tabulations (Abramowitz and Stegun, 1964; Davis, 1963) and agree with all the digits available ( 10 to 14 significant digits).

-Research sponsored by Applied Mathematical Sciences
Research Program, U.S. DOE Office of Energy Research. 8.70

\section{APPROXIMATIONS TO DISTRIBUTIONS*}

\section{K. O. Bowman}

(Abstract of paper presented at the University of Tokyo, Tokyo, Japan, May 20, 1985)

We shall consider methods of approximating the distributions of statistics using first four moments, the moments being derived from Computer Oriented Extended Taylor Series (COETS) algorithms. Frequently, series are divergent and may require the use of numerical summation algorithms.

- Research sponsored by Applied Mathematical Sciences Rescarch Program, U.C. DOE Office of Energy Rescarch. 


\section{BOUNDS FOR THE EXPECTATION OF THE kth ROOT OF A RANDOM VARIABLE*}

\author{
K. O. Bowman H. K. Lam ${ }^{t}$ \\ L. R. Shentongt
}

[Abstract of J. Comp. Appl. Math. 10, 229 (1984)]

Exact bounds for the mean value of a fractional moment, such as the sample standard deviation, are considered. These bounds are compared with those 'ound by summation techniques applied to computer extended series. An expansion involving an arbitrary parameter is introduced, and now best bounds are found by optimization. There are applications to bounds for a certain class of quadrature problem.

- Research sponsored by Applied Mathematical Sciences Research Program, U.S. DOE Office of Energy Research.

'Department of Statistics, The Chinese University of Hong Kong, Shatin, N.T., Hong Kong

IAdvanced Computational Methids Center, University of Georgia. Athens, GA.

\subsection{2}

\section{BOUNDS FOR CERTAIN INTEGRALS*}

\section{K. O. Bowman H. K. Laxi}

\section{R. Shenton?}

[Abstract of J. Comp Appl Marh. 10. 245 (1984)]

A certain class of definite integrals is considered in which the integrand consists of ? onesigned function together with another function which has a one-signed derivative in a certain interval. By examining the Cauchy form of the remainder, sets of bounds are developed which have a certain optimum property. The integrals may be multi-dimensional. The case in which the derivative component is not one-signed is briefly considered.

\footnotetext{
- Research sponsored by Applied Mathematical Sciences Research Program, U.S. DOF: Office of Energy Research.

Department of Statistics. The Chinese University of Hong Kong, Shatın, N.T., Hong Kong.

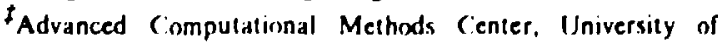
(ierstria, Athens, (iA
}

\section{BOUNDS FOR INTEGRALS USING SEGMENTS*}

\author{
K. O. Bowman H. K. Lam ${ }^{\dagger}$ \\ L. R. Shenton ${ }^{\ddagger}$
}

[Abstract of J. Comp. Appl. Math 11, 57 (1984)]

For certain integrals, with one-signed integrands, bounds depending on a parameter can be constructed. Sharpness of the bounds can be improved by fragmentation of the interval; this device is the basis of most quadrature formulas. -Research sponsored by Applied Mathematical Sciences
Research Program, U.S. DOE Office of Energy Research.
'Department of Statistics, The Chinese Universily of Hong
Kong. Shatin, N.T., Hong Kong.
tAdvanced Computational Methods Center, University of
Georgia, Athens, GA.

8.74

\section{THE DISTRIBUTION OF MOMENT ESTIMATORS FOR PARAMETERS OF THE GENERALIZED POISSON DISTRIBUTION*}
K. O. Bowman
L. R. Shenton't

(Abstract of paper to be presented dt the Annual Joint Meeting of the American Statistical Association, Institute of Mathematical Statistics Biometric Society, Las Vegas, NV, August $58,[985]$

There are many cas ss for which a theoretical expression for a high-order term in the expectation of a Taylor series for a moment ratio is out of reach. When the series shows strong divergent tendencies, loss of accuracy, especially in coefficients of $n^{-15}$ and above, is to be expected. Although this may be taken care of for isolated cases, it can be very time consuming. Our example provides the general coefficient in the series for the mean and variance, so that a check of the computer-oriented extended Taylor series (COETS) approach is possible.

A nother check on the approach by divergent scries and four-moment distributional approximations shows threc different estimators of a parameter to be in excellent agreement. 
- Research sponsored bs Applied Mathemutical Sciences Research Program, U.S. DOE Oftice of Energy Research.

'Advanced Computationa: Methods Center, University of Georgia, Athens, GA

8.75

\section{THE DISTRIBUTION OF A MOMENT ESTIMATOR FOR A PARAMETER OF THE GENERALIZED POISSON DISTRIBUTION*}

\section{K. O. Bownan L. R. Sweanton ${ }^{t}$}

[Abstract of Communication in Statistics Joumal (in press)]

The ratio of the sample variance to the sample mean estimates a simple function of the parameter which measures the departure of the Poisson-Poisson from the Poisson distribution. Moment series to order $n^{-24}$ are given for related estimators. In one case, exact integral formulations are given for the first two moments, enabling a comparison to be made between their asymptotic developments and a computer-oriented extended Taylor series (COETS) algorithm. The integral approach using generating functions is sketched out for the third and fourth moments. Levin's summation algorithm is used on the divergent series and comparative simulation assessments are given.

- Research sponsored by Applied Mathematical Sciences Research Program, U.S. DOE Office of Energy Research.

'Advanced Computational Methods Center, University of Georgia, Athens, GA.

\subsection{6}

\section{THE DARK SIDE OF ASYMPTOTICS AND MOMENT SERIES*}

\section{K. O. Bowman \\ L. R. Shenton ${ }^{\dagger}$}

[Abstract of Journal Sankhyd (in press)]

The nature of series for moment estimators of the parameters of the negative binomial distribution is used to highlight the part played by basic first order asumptotics in more general situations and the risks incurred when these are used as numerical assessments. Algorithmic procedures are described.

- Research sponsored by Applied Mathematical Sciences Research Program, U.S. DOE OTice of Energy Research.

'Advanced Computational Methods Center, University of Georgia, Athens, GA.

8.77

\section{STUDY OF AGE DEPENDENT HALF-LIFE \\ OF IODINE IN MAN: A REINFORCEMENT- DEPLETION URN MODEL *}

\author{
K. O. Bowman L. R. Shenton' \\ S. R. Bermard ${ }^{t}$
}

[Abstract of Bull. Math Biol. 47, 205 (1985)]

An urn contains balls of $s$ different colors. The problem of the reinforcement of a specified color and random depletion of balls has been considered by Shenton (1981, 1983). In this paper, the theory is applied to the biological age dependent half life of radioiodine in man; the data of Cook and Snyder (1965) are used. The intake of radioiodine and its retention subsequently are studied.

- Research sponsored by Office of Health and Environmental Research, U.S. DOE Office of Energy Research.

Advanced Computational Methods Center, University of Georgia, Alhens, GA.

$t_{\text {Health and Safety Division. }}$

\subsection{8}

\section{A REINFORCEMENT-DEPLETION URN MODEL - A CONTIGUITY MOUEL*}

\section{K. O. Bowman L. R. Sheatoa ${ }^{\dagger}$ \\ S. R. Bernurd \\ [Abstract of Ann. Inst. Stat. Maih. (in press)]}

An urn contains balls of $s$ different colors. The problem of the reinforcement of a specified color and random depletion of balls has been considered by Bernard (1977) and Shentun 
(1981.1983). Here we consider a special relation between a reinforcement and depletion, leading to a hypergeometric distribution.

- Research sponsored by OTice of Health and Environmental Research, U.S. DOE Office of Energy Research.

Advanced Computational Methods Center, U-i.ersity of Georgia, Athens, GA.

$t_{\text {Health and Safety Division. }}$

8.79

\section{A POISSON SUM UP TO THE MEAN AND A RAMANUJAN PROBLEM*}

\author{
K. O. Bowman L. R. Shenton ${ }^{\dagger}$ \\ G. Szekerest
}

[Abstract of J. Stat. Comp. Simul. 20, 167 (1984)]

Ramanujan considered a large number of mathematical problems, ranging from moderately simple to extremely difficult. A simple one is concerned with the exponential function $\exp (\zeta)$ when $\zeta>0$ and gives the usual series with an unusual remainder term. He considered the function $y(x)$ defined by

$$
\begin{gathered}
1 / 2 \exp x=1+x+x^{2}, 2 !+\ldots+x^{x-1} /(x-1) ! \\
+x^{x} y(x) / x !
\end{gathered}
$$

where $x$ is a large positive integer, showing that

$$
\begin{gathered}
y(x)=1 / 3+4 /(135 x)-8 /\left(2835 x^{2}\right) \\
-16 /\left(8505 x^{3}\right), x \rightarrow \infty .
\end{gathered}
$$

Does the series diverge, and is the sign pattern of periud four sustained? Evidence from extended series to :!: $=$ term $x^{-15}$ suggests answers in the affirmative. Furthermore, factoring the cuefficients from the computer output led to the conjecture $x$ value $2^{7} \cdot 5459 /\left(3^{10} \cdot 5^{2} \cdot 7 \cdot 11 \cdot 13\right)$ for the coefficient of $x^{-6}$. Using subjective probability in pure mathematics, Jack Good supported the conjecture.

- Research sponomed by Applied Mathematical Sciences Research Program. U.S. IO)E Office of Energy Research.

Advanced Compulational Methods (enter. Inniversity of (jeorgia, Athens, (;A.

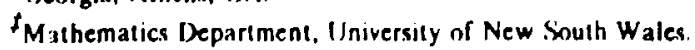

8.80

\section{FUZZY HIERARCHICAL ANALYSIS*}

\author{
J. J. Buckley ${ }^{\dagger} \quad$ V. R. R. Uppeluri
}

(Abstract of paper presented at 1984 Annual Meeting of the Society for Rist Analysis, Knoxville, TN, September 30 October 3, 1984; also at Statistics Symposium on National Energy Issues, Seattle, WA, October 16-18, 1984)

This paper extends hierarchical analysis to the case where the participants are allowed to employ fuzzy ratios in place of exact ratios. If a person considers Alternative A more important than Alternative $B$, then the ratio used might be approximately 3 to 1 , or between 2 to 1 and 4 to 1 , or at most 5 to 1 . The pairwise comparison of the issues and the criteria in the hierarchy produce fuzzy positive reciprocal matrices. The geometric mean method is employed to calculate the fuzzy weights for each fuzzy matrix, and these are combined in the usual manner to determine the final fuzzy weights for the alternatives. The final fuzzy weights are used to rank the alternatives from highest to lowest. The highest ranking contains all the undominated issues. The procedure easily extends to the situation where many experts are utilized in the ranking process. Two examples are presented showing the final fuzzy weights and the final ranking.

- Research sponsored by the Nuclear Regulatory Commission and U.S. Department of Energy.

University of Alabama, Birmingham, AL.

\subsection{1}

\section{QUANTITATIVE ASPECTS OF METAL ION CONI ENT AND TOXICITY IN DROSOPHILA *}
N. T. Christie ${ }^{\dagger}$
D. G. Cosslee
L. C. Batet
K. B. Jacobsons
[Abstract of Toxicology 26, 295 (1983)]

As a basis for both a genetic and a biochemical approach to a study of metal ion effects, a method for quantitating the toxic response of Drosophila to metal ions was developed. The response to 13 metal ins has been examined, 
including several chemical groups from the periudic table: the IIb ions $\mathrm{Zn}^{2+}, \mathrm{Cd}^{2+}$, and $\mathrm{Hg}^{2+}$; the Ila ions $\mathrm{Be}^{2+}, \mathrm{Mg}^{2+}, \mathrm{Sr}^{2+}$, and $\mathrm{Ba}^{2+}$; the transition elements $\mathrm{Ni}^{2+}, \mathrm{Cu}^{2+}, \mathrm{Co}^{2+}$, and $\mathrm{Mn}^{2+}$; and trivalent ions $\mathrm{Y}^{3+}$ ar:d $\mathrm{Cr}^{3+}$. The standard test procedure prevides estimates of the median lethal concentration $\left(\mathrm{LC}_{50}\right)$ and the range of the tolerance distribution, both of which are obtained by the method of probit transformation. Range is defined as the change in concentration between the $\mathrm{LC}_{2.5}$ and the LC $\mathrm{L}_{97.5}$. Estimation of range as the measure of the limits of the tolerance distribution was utilized to measure variation in the response to metals with time. Genetic characterization of strains will require strains of flies without overlapping tolerance ranges. Although there was a general trend of increasing values of range with increasing $\mathrm{LC}_{50}$ values, the exceptions (e.8., $\mathrm{Cr}^{3+}$ and $\mathrm{Be}^{2+}$ ) indicated that several factors may be involved in determining the range of lethal responses. Elemental analyses have been performed on flies befoie and after metal ion treatments to establish the amount of metal ion taken up and baselines for comparison. This study provides additional evidence that Drosophila is an appropriate organism for the study of specific biochemical alterations induced by metals, e.g., the cadmium-induced increase in $\mathrm{Q}(+)$ tRNAs.

- Research sponsored by U.S. DOE Office of Health and Environmental Research.

University of Tennessee-Gak Ridge Graduate School of Biomedical Sciences.

$\ddagger_{\text {Analytical Chemistry Division. }}$

\$Biology Division.

\subsection{2}

\section{REGRESSION METHODS FOR BINOMIAL AND POISSON DISTRIBUTED DATA*}

\section{E. L. Frome}

(Abstracl of a paper presented at the American Association of Physicists in Medicine First Midyear Topical Symposium on Multiple Regression Analysis: Applications in the Health Sciences. Mobile. AL, March 12 16, 1984)

Models are considered in which a rate or probability can be represented by a regression function that describes the relation between the predictor variables and the unknown parameters.
Estimates of the parameters can be obtained by means of iteratively reweighted least square (IRLS). When the dependent variable is a count that follows either the Poiscon or binomial distribution, the IRLS algorithm is equivalent to using the method of scoring to obtain maximum likelihood (ML) estimates. This general least squares regression approach includes linear, generalized linear, and intrinsically nonlinear regression functions. Standard statistical packages that support IRLS can be used to obtain ML estimates, their asymptotic covariance matrix, and diagnostic measures that can be used to aid the analyst in detecting outlying responses and extreme points in the model space. The results of fitting several different models to the same data set can be summarized in an ANOVA-like table using the deviance as a measure of residual variation. Five examples using data from both designed experiments and observational studies are presented to illustrate the utility of Poisson and binomial regression analysis.

- Research sponsored by Applied Mathematical Sciences Research Program, :IS. DOE Office of Enetgy Research.

\subsection{3}

\section{RESPONSE TO NELDER'S REACTION ON POISSON RATE ANALYSIS*}

\author{
E. L. Frowe
}

[Abstract of Biometrics Journal 40, 1160(1984)]

Computational issues that arise when fitting regression models to Poisson distributed data are discussed. Iterative weighted least squares (IWLS) is used to obtain maximum likelihood estimates for general nonlinear regression functions. For generaliz id linear regression functions, the IWLS procedure can be modified to take advantage of special structures in the likelihood equations. These results are made in response to comments by J. A. Nelder on a recent paper in Biometric.s.

- Research spunsored by U.S. DOE Office of Health and Environmental Rexearch. 


\section{THE USE OF POISSON REGRESSION MODELS IN ESTIMATING INCIDENCE RATES AND RATIOS*}

\author{
E. L. Frome H. Oreckoway ${ }^{\dagger}$
}

[Abstract of Amer. J. Epidemiolozy I21(2), 309 (1985)]

Summarizing relative risk estimates actoss strata of a covariate is commonly done in comparative epidemiologic studies of incidence or mortality. Conventional Mantel-Haenszel and rate standardization technigues used for this furpose are strictly suitable only when there is no interaction between relative risk and the corariatc, and tests for interaction typically are lir.ited to examination for departures from linearity. Poisson regression modeling offers an alternative technique which can be used for sunmarizing relative risk and for evaluating complex interactions with covariates. A more general application of Poisson regression is its utility in modeling disease rates according to postuiated etiologic mechanisms of exposures or according to disease expression chancteristics in the population. The applications of Poisson regre:sion analysis to problems of summarizing relative risk and disease rate modeling are illustrated with examples of cancer incidence and mortality data, including an example of a nonlinear model predicted by the multistage theory of carcinogenesis.

-Research sponsored by U.S. DOE Office of Health sind Envifonmental Research and by Oak Ridge Ascocizied Universities.

'Department of Epidemiology. University of North Carolina.

\section{APPLICATION OF POISSON REGRESSION TO THE ANALYSIS OF CHILDHOOD LEUKEMIA DEATHS IN UTAH*}

\author{
E. L. Frome $\quad$ D. L. Craglet
}

(Abstract of paper to te presented at the Annual Joint Meeting of the American Statistical Association, Institute of Mathematical Statistics Biometric Society, Las Vezas, NV August 5-8, 1985)

In epriemiologic follow-up studies incidence or mortality data are often summasized in a multidimensional table. The entries in each cell of the table are the number of cases and personyears. The problem of interest involves leukemia deaths in children in the state of Utah from 1944 to 1975. Four factors are considered in the analysis: age, sex, location (north vs. south) and time period (before, during, and aftur nuclear testing). Poisson regression models are used to describe the disease rates as a function of the factor levels. The importance of the factors is assessed in terms of their ability to "explain variation" in the data. Our reanalysis of these data indicate that "age-adjusted' rates are not appropriate for this situation. The results are of methodologic interest since they illustrate procedures for dealing with some of the difficulties of model selection that may occur in multivariate studies.

\footnotetext{
- Research sponsored jointly by U.S. DOE Office of Health and Environniental Research and the Oak Ridge Associated Universitic:

'Oak Ridge Associated Universities, Oak Ridge, TN.
} 
8.86

\section{A MACRO FOR FTTTING NONLINEAR MODEIS TO POLSSON DISTRIBUTED DATA*}

\author{
E. L. Frome R. Melain
}

|Abst:act of paper presented at the Ninth SAS Users Group International Conference, Cary. NC. March 18-21. 1984: Froc. Sir: Anal. Syst. Users Group Int., p. 883 (1984))

Models are considered in which the dependent variable $y$ is a count that follows the Poisson distribution. The expected value of $y$ is represented with a regression function that describes the relation between the expected count, the prodictor variables, and the parameters. Estimates of the parameters can be obtained using iteratively weighted least squares (IWLS). The IWLS procedure is equivalent to using the method of scoring to obtain a root of the likelihood equations. Poisson regression models include linear, loglinear, quasi-linear, and intrinsically nonlinear regression functions. This approach allows the analyst to concentrate on determining the most appropriate form of the regression function without regard for computational complexity. The SAS procedures NLIN and MATRIX can be used to obtain ML estimates, their estimated asymptotic covariance matrix, and diagnostic measures that can be used to aid the analyst in detectung outlying responses and extreme points in the model space. An example, using an intrinsically nonlinear model (derived from the theory of Dual Radiation Action), is used to illustrate the ML estimation, hypothesis testing, and regression diagnosties techniques.

- Research sponsored by Applied Mathematical Sciences Research Program, U.S. DOE OrTice of Energy Rescarch.

'Oak Ridge Aseociated Universities.
8.87

\section{RESPONSE OF MOUSE SPERMATOGONIAL STEM CELS TO X-RAY INDUCTION OF HERTTABLE RECIPROCAL TRANSLOCATIONS"}

\author{
W. M. Generoso" \\ K. T. Csin't \\ N. L. A. Cactuiro' \\ C. V. Connett \\ D. G. Gasilee
}

[Abstract of Mutation Reseer ih 126, 177 (1984)]

Although heritable translocations are an important endpoint for the assessment of genetic risk from radiation, there has been a serious information gap - jth regard to their induction in spermatogonial stem cells, the most important cell stage in males for risk considerations. This led to uncenainty in estimating the magnitude of risk per unit exposure. Further, the relationship between the frequency of reciprocal exchanges scored by cytological analysis of the exposed male's meiocytes and the frequency of those transmitted to first-generation offspring needed to be re-examined. In order to fill in these gaps, two radiation studies, i.e., dose response and dose fractionation, were conducted on spermatogonial stem cells in which heritable and cyrologically detected translocations were scored.

The present data are by far the most extensive, to date, for heritable translocation induction in spermatogonial stem cells. The linearity of the rising portion of the dose-effect curve and the additivity of effects observed in the fractionation study allow a direct estimation of the number of transmissible translocations expected per unit exposure. Thus, the expected increase in heritable transincations per rad of acute $X$ rays is 3.89 $\times 10^{-5}$ per gamete. The data also show a lack of consistency between cytologically and genetically scored translocations.

\footnotetext{
- Recearch sponeored by U.S. DOE OTice of Health and Environmental Research.

${ }^{\text {PBolozy Division. }}$
} 


\section{DIFFERENCE IN THE RESPONSE OF TWO HYBRID STOCKS OF MICE TO X-RAY INDUCTION OF CHROMOSOME ABERRATIONS IN SPERMATOGJNIAL STEM CELLS}

\section{W. M. Generosot K. T. Coint \\ C. V. Connett ${ }^{\prime}$ N. L. A. Cacteim' \\ L. A. Hoghes' P. W. Bradea'}

D. G. Gonder

[Abstract of Mulation Resear:h (in preas)]

lonizing radiations induce balanced reciprocal translocations in spermatogonial stem cells of mice. From ceils carrying these rearrangements, which can be scored cytologically in the diakinesis-metaphase I stage, balanced normal, balanced translocated and unbalanced (duplication/deficiency) sperm can be produced. The relationship between expected (calculated from cytological data) and oisserved frequencies of embryonic lethality (presumably as a result of unbalanced sperm fertilizing the egg) following exposure of spermatogonial stem cells to $X$ rays was studied in two hybrid stocks. A marked difference in the incidence of induced embryonic lethality was found between the two stocks Similarly, a difference in the cytological frequen. cies of translocations was also found, although smaller than that observed for embryonic lethal ity. Thus, it appears that the difierence between the two stocks in the frequencies of embryonic lethality may be attributable both to processes occurring prior to metaphase I and to a difference in the rate of transmission of unbalanced chromosome constitutions.

- Research sponsored by U.S. DOE Orfice of Healih and Environmental Research.

'Biolocy Division.

\section{ESTIMATION OF THE MINIMUM LATENT PERIOD FOR RADIOGENIC OSTEOSARCOMAS IN MAN*}

\author{
P. G. Groet $\quad$ V. R. R. Unpmari
}

(Abstract of paper presented at Symposium on the Radiobiology of Radium and Thorotrast, Neuberberg. Fedieral Republic of Germany, October 29 \$1, 1984)

The latent period $t$ for radiogenic osteosarcomas and radiogenic cancers in general can be thought of as the sum of two unknown quantities: the growth period of the tumor $(\gamma)$ and the induction perioa $i \eta)$. The latter is the time from first exposure to transformation of the first isormal cell that develops into a detectable tumor n.ass during $\gamma$. Neither the induction period nor the growth period are directly observatle. But it is possible to estimate $\gamma$ and $\eta$ from observations of $\tau$ if the osteosarcoma rate does not depend on the lime since first exposure. We use data on ${ }^{226}$ Ra-dial painters with estimated initial intake greater than $230 \mu \mathrm{Ci} / \mathrm{kg}$ bone to determine the posterior distributions of the growth period $x(\lambda D)$ given the data $D$. The data $D$ consist of the times to tumur diagnosis and censoring. W'e found a posterior mean gre!:th period of 5.5 years for these dial painters. This is the mean minimum latent period for radiogenic csteosarcomas in ma-. The approach described in this paper needs the assumption that all osteosarco mas diagnosed in this group of dial painters are radiation induced. This is quite reasonable, since the probability of a spontaneous osteosarcoma in this group given a rate of about $10^{-6}$ (person-years) $^{-1}$ and a total time at risk of 764 person-years is less than one in a thouszond.

- Revearch sponeored by U.S. DOE Orince of Research and Development and Ofisce of Health and Environmental Research.

'Oak Ridge Aseaciated Univervitiea, Oak Ridge, TN. 


\section{STATISTICAL EVALUATIONS IN THE CARCINOGENESIS BIOASSAY OF PETROLEUM HYDROCARBONS*}

\author{
J. M. Holland ${ }^{\prime}$ E L Frome
}

(Abaract of Chapler 12 in Applied Toxicolozy of Petrolewn Hudrocartions, Vol. VI. pp. 151. Princeton Scientific Publisb. ers, Princeton, NJ. 1984)

Petroleum has repeatedly been shown to be a skin carcinogen in animal experiments. Given the chemical complexity of natural petroleum, it is unlikely that carcinogenic action can be quantitatively correlated with any single chemical class. The chemical matrix as well as the kind and amount of polyaromatic hydrocarbons (PAH) contribute to the skin carcinogenic potential of fossil liquids. For these reasons it will be necessary to continue to rely upon animal bioassays until in vitro methods have been established that reflect this complexity.

Work done at Oak Ridge National Laboratory over the past several years has focused on the question of carcinogenic potency and its evaluation. While the emphasis of this work has been on synthetic fossil liquids, we also hare had the opportunity to apply our methods to an extensive data base developed by the Kettering Laboratory under contract with the American Petroleum Institute. This presentation describes the methods we have developed to determine skin carcinogenic potency and illustrates the application of these methods by an analysis of the API/Kettering data base.

-Rescarch sponsored by I'S. DOE Orisce of Health and Environmental Recearch.

'Chevron Environmental Health Center, Richmond, CA.

\section{A STOCHASTIC MODEL FOR ESTTMATION OF ENVIRONMENTAL DENSTTY*}

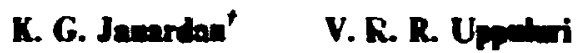

(Abstract of paper presented at Modeling and Simulation Conference. Pittsborgh, PA. April 19-20, 1984)

The environmental density has been defined (Morisita, 1971) as the value of a habitat expressing its unfavorableness for settling of an individual which has a strong anti-social tendency to other individuals in an environment. Morisita studied anti-social behavior of ant-lions (Glemuroides japanicus) and provided a recurrence relation without an explicit solution for the probability distribution of individuals settling in each of two habitats in terms of the environmental densities and the numbers of individuals introduced. In this paper the recurrence relation is explicitly solved; certain interesting properties of the distribution are discussed including the estimation of the parameters.

- Research sponsored by U.S. Department of Energy.

'North Dakota State Univeraity, Fargo, ND.

8.92

\section{INHALATION TOXICOLOGY OF DIESEL FUEL OBSCURANT}

AEROSOL IN SPRAGUE-DAWLEY RATS*
S. Lock'
W. Dalbey'
R. L. Schwoyer
R. Griesener'

(Abstract of ORNL/TM-9403, May 1984)

Inhalation exposures were performed twice per week for 13 weeks to determine whether there was any potential toxicity to rats of comparatively low concentrations of a condensation aerosol from diesel fuel. Animals were divided into four groups (24 per sex in each group) and exposed to aerosol concentrations of $0,0.25,0.75$, and $1.50 \mathrm{mg}$ diesel fuel aerosol/L for four hours per day. A fifth group ( 12 per sex) was used as 
vivarium controls. Body weight and food consumption were measured weekly for duration of the exposure and also during a two-month recovery period. Changes in breathing frequency and the response of animals to a loud sharp sound (startle response) were measured in selected animals prior to the start of the exposures, at various time points during the 13-week exposure period, and at monthly intervals duing the recovery period. Assays were performed on selected animals at the end of the exposure period and again after the iwo-month recovery period. Endpoints included pulmonary function tests, numbers of alveolar free ce!'s, clinical chemistry, hematology, organ weights, and histopathology.

Nu mortalities were recorded during the exposure of recovery periods. Slight toxicity occurred at these low aerosol concentrations with the loss in body weight of all treated animals Juring the exposure period. During the exposure period there were also some slight changes in startle reflex: however, these were apparently acute effects, and there appeared to be no permanent CNS involvement as measured by this endpoint. Immediately post-exposure, the numbers of lavaged alveolar macrophages were slightly elevated in all acrosol exposed animals. Pulmonary function tests, pulmonary gas exchange and dynamic lung tests were all apparently unaffected by these low diesel fuel aerosol exposures. Changes in tissue weights in aerosol exposed animals were minor, and the few histopathological lesions were randomly scattered among all groups included in this study and were more attibutable to the age of the animals than any specific treatment group. No significant cumulative toxicity may be attributed to these diesel fuel aerosol exposures.

- Research sponsored by U.S. Army Medical Research and Development Command through the ORNL Biolong Division.

'Biology Division.
8.93

\section{FIRST DIGITS OF EXPONENTIALLY DISTRIBUTED RANDOM VARIABLES*}

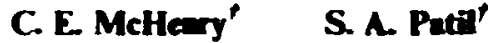 \\ V. R. R. Unmari
}

i Abstreat of Anaual Mecting of the Teanesuce Academy of Sciences. Mathematics Section, Krouville TN, Nosvember 16 17. 1984]

The first nonzero digit $k$ of a random number $x$ drawn from a distribution that is uniformly distributed on $(0,1)$ takes on the nine possible values 1, 2, .., 9 with equal probability 1/9. Recently, Uppuluri and Patil found the probability distribution of the first nonzero digit of the reciprocal of a number drawn at random from a distribution that is uniformly distributed on $(0,1)$. These results were also generalized by the same authors to the case of beta-distributed random variables. Since the infinite series derived for these problems were expressible in closed forms, neat results were obtained even in the case of the probability distribution of the first $j$ digits when a number is drawn at random from a beta distribution.

In the case of the exponential distribution, the probability distribution of the first nonzero digit could not be expressed in closed forms; therefore, numerical techniques were used to determine approximations to the desired probabilitie: Similar results were obtained for the first nonzero digit of a number drawn at random from the distribution of the ratio of two i.i.d. exponential random variables. In addition, Monte Carlo studies were done for comparison purposes.

\footnotetext{
- Research sponsored by Oak Ridge National Laborat ry.

'Tennessee Technotonical Universily. Cootevilie. TN.
} 
8.94

\section{APPLICATION OF MAXIMUM LIKELHOOD PAIRED COMPARISON RANKING TO ESTTMATION OF A LINEAR LOMINANCE HIERARCHY' IN ANIMAL SOCTETIES*}

\author{
$\begin{array}{ll}\text { C. A. MoMalan't } & \text { M. D. Morris }\end{array}$ \\ [Abatrect of . Inim Betwor. 32, 374 (1984)]
}

Dominance hierarchies have been widely used for describing the outcome of $c$ mpetitive interactions in an animal group. We present a procedure for estimating the linear dominance hierarchy. The procedure uses the statistical method of paired comparisons, assuming weak stochastic transitivity to model interactions within a linear dominance hierarchy. The linear dominance hierarchy is estimated using a maximum likelihood ranking procedure. This method allows unequal numbers of encounters between pairs and does not require all pairs to have observed encounters. The method is illustrated by application to behavioral data from a group of ten baboons (Papio cynocephalus anubis).

-Resarch sponsored by U.S. DOE Ottice of Healib and Environmental Recearch.

'Deparumeat of Pathology. Univeryity of Texas Health Science Center, San Antonio, TX.

\subsection{5}

\section{COMPUTER CONSTRUCTION OF SUPERSATURATED SCREENING DESIGNS*}

\section{T. J. Mitchell}

(Abatract of paper presented at Workshop on Efficient Data Collection, Univernity of Californie, Berteley, CA. February 11-13, 1985)

In a "screening" experiment, there are many controllable variables, but very few are expected to affect the outcome. The objective is to find them. When it is not feasible to conduct as many experimental trials as there are variables, a "supersaturaied" experiment is indicated. This paper presents a Bayesian approach to the analysis and design of such experiments. The design criterion is to maximize the information (defined formally in terms of entropy) in the posterior probability disiribution of the competing models. Computer algorithms to search for optimum designs will be discussed, and some examples given.

- Research sponsored by Applied Mathematical Sciences Research Program. U.S. DOE Office of Energy Research.

\subsection{6}

\section{MINMUM NUMBER OF RUNS \\ FOR TWOLEVEL FACTORIAL SEARCH DESIGNS*}

\section{D. Maria}

[Abaract of J. Siaristical Planving and Inference 10, 115 (1984)]

A lower bound is given for the number of experimental runs required in search designs for two-level factorisl models.

- Reacarch sponsored by Applied Mathemalical Sciences Research Program, U.S. DOE Office of Energy Researct.

8.97

\section{AN INTERESTING PROPERTY \\ OF THE SAMPLE MEAN UNDER A FTRST-ORDER AUTOREGRESSIVE MODEL*}
M. D. Morris
S. F. Ebey ${ }^{t}$

[Abatract of The American Siatistician 342), 127 (1984)]

The unweighted sample mean is examined as an estimator of the population mean in a firstorder autoregressive model. It is demonstrated that the precision of this estimator deteriorates as the number of equally spaced observations taken within a fixed time interval increases.

\footnotetext{
-Reacarch sponsored by U.S. DOE Ofrice of Health and Environmental Research.

Department of Mathematica, Univerity of the South, Sewanee. TN
} 
8. 8

\section{ON THE PRECISION OF THE AVERAGE OF CORRELATED MEASUREMENTS*}

\author{
M. D. Morris S. F. Ekey
}

(Aburact of paper presented at Biometry Branch Seminar, National Institute of Environmental Health Science Research, Triangle Part. NC. Apri) 13. 1984)

In many biological and medical contexts, the unweighted average of several repeated measurements is used as the estimate of an unknown parameter value. We examine the precision of this statistic when the individual measurements are realizations of a first-order autorcgressive time series plus independent "white noise." We demonstrate that under certain cunditions, the precision of the average dusreases with an increase in the number of evenly spaced observations with:n a fixed time interval. Also, we characterize optimal spacing of measurements within a fixed interval.

- Research sponsored by U.S. DOE Office of Health and Environmental Research.

Department of Mathematics, University of the South, Sewanee, TN.

\section{ANOVA MODEL FITTING VIA SPARSE MATRIX COMPUTATIONS*}

\section{G. Ostronchov}

(Abstract of paper to be presented at the Annual Joint Meeting of the American Statistical Areociation. Institute of Mathematical Statistics Biometric Sociely, Las Vegas, NV, Augu'st 58,1985 )

Full matrix methods for fitting large unbalanced ANOVA models require enormous amounts of time and storage. The model matrix $X$ (often called the design matrix) consists of dummy variables and is sparse; thus great reduction in time and storage can be achieved by viewing this as a sparse matrix problem. A m:athod, based on orthogonal Givens factorization of a maximal rank set of sparse columns of $X$, for fitting a model and computing the residual sum of zquares is presented. The selection of a maximal rank set of sparse columns is based on a description of linear dependencies between columns of $\boldsymbol{X}$ that is developed using a notation based on index sets associated with model terms. A comparison of the new algorithm to a full matrix algorithm on a set of models shows that both time and storage requirements are reduced by up to three orders of magnitude for large models and are competitive for small models.

-Research sponsered by Appliod Mathematical Sciences Research Program. U.S. DOE OfTice of Energy Research.

\subsection{0}

\section{LARGE SPARSE LEAST SQUARES COMPUTATIONS*}

\section{G. Ostronchor}

(Abstract of thesis for Doctor of Philosophy. Iowa State University, Ames, lowa, 1984]

Orthogonal Givens factorization is a popular method for solving large sparse least squares problems. Row and column permutations of the data matrix are necessary to preserve sparsity and reduce the computational effort during factorization. The cumputation of a solution is usually divided into a symbolic ordering phase and a numericai factorization and solution phase. Some theoretical results on row ordering are obtained using a graph-theoretic representation. These results provide a basis for a symbrolic Givens factorization. Column orderings are also discussed, and an efficient algorithm for the symbolic ordering phase is reveloped. Sometimes, due to sparsity considerations, it is advantageous to leave out some rows from the factorization and then update only the solution with these rows. A method for updating the solution with additional nws or constraints is extended to rank-deficient problems. Finally, the application of sparse matrix methods to large unbalanced analysis of variance problems is discussed. Some of the developed algorithms are programmed and tested.

- Research sponsored partially by Applied Mathematical Sciences Revearch Program, U.S. DOE Office of Encrity Research. 
8.101

\section{SYMEOLIC GIVENS REDUCTION IN LARGE SPARSE LEAST SQUARES PROBLEMS*}

\section{G. Ostronedor}

(Abatract of ORNL/TM-6102, December 1S84; abo SIAM J. Sci. Star. (in press), aboo peper presented at the Society for Industrial and Applied Mathematica National Meeting. University of Washington, Seattle, WA, July 16-20, 1984]

Orthogonal Givens factorization is a popular method for solving large sfarse least squares problems. To exploit sparsity and to use a fixed data structure in the factorization, a "good" row and column ordering needs to be found and a preliminary symbolic factorization needs to be performed. Some results on row-ordering and structure of rows in a partially reduced matrix are ohtained using a graph-theoretic representation. These results provide a basis for a symbolic Givens factorization. Column-ordering is also discussed, and an algorithm for symbolic Givens reduction is developed and tested.

- Research sponsorod by Applied Mathematical Sciences Research Program, U.S. DOE Otifice of Energy Research.

8.102

\section{EFFECT OF MULTIPLE SEQUENTIAL THYMUS GRAFTING ON IMMUNE COMPETENCE AND MEAN LIFE SPAN*}

\author{
$\begin{array}{ll}\text { E. H. Perkinst } & \text { P. L. Glovert }\end{array}$ \\ D. G. Gocalee \\ [Abatract of J. of Gerontology (in press)]
}

A thymus from a syngeneic newborn, grafted under the kidney capsule, grows and differentiates as adequately in old as young-adult mice, but following maturation the older the animal the more rapid the involution of the transplanted thymus. The growth kinetics and histological appearance of a second thymus graft is independent of the presence of the first graft. We ionfirm that thymus grafting partially restores immune competence, however, the significance of this restoration as it may relate to an increase in the median life span has never been assessed. We now report that multiple sequential thymus grafting at certain ages and with proper intervals beiween the first and second thymus graft appears to sufficiently preserve health to significantly increase median life span. Postulating that immunological rejuvenation plays a significant role in the observed increase of median life span, greater credence is given to an enhanced resistance against infectious rather than malignant disease. However, since only 20-30\% of aged animals die from infections, the reported data appear to be of considerable significance and may implicate partial restoration of other physiological parameters in addition to immune competence.

-Research sponsored by U.S. DOE OfTice of Healih and Environmental Research.

'Biology Division.

I National Institute of Environmental Health Sciences, Research Triangle Park. NC.

\subsection{3}

\section{SOFTWARE SYSTEMS FOR STATISTICAL PROCESS CONTROL AND SIMULATED- PROCESS OPTIMIZATION*}

\section{C. Selubtzhaser}

(Abstract of paper presented at the Ellis R. Ou Conference, New Brunswick, NJ, March 18-21, 1985)

As more processes are stabilized through the application of control charts, statistically designed experimentation plays a larger role in process improvement. But it is the combination of statistical tools - control charts and experimental design - that yields the biggest effect on process quality. Acceptance of these methods in industry can be greatly enhanced by involving computers. The key is software.

The simple calculations needed to produce control charts have made these tools available to production personnel. A prompt-driven software system makes control charts even more accessible - and to all levels of a company. However, the statistical design of experiments has always 
been more difficult for the non-statistician to grasp. Software can be used here in the form of a process-simulating game to be played by both experimenters and statisticians. In this way, we can demonstrate through experience the value of designed experiments. Two software systems written using SAS and based on these ideas will be discussed.

-Research sponsored by the Quality Division. Y-12 Flant

\subsection{4}

\section{SIGMOIDALLY CONSTRAINED MAXIMUM LIEELIHOOD ESTIMATION IN QUANTAL "MASSAY*}

\section{R. L. Schoojer}

[Abaract of J. Am. Stat. Assac. 79, 448 (1984)]

In quantal bioassay, doses $x_{1} \ldots ., x_{k}$ of a test substance are administered to $n_{1}, \ldots, n_{k}$ animal subjects. Let $\Pi(x)$ denote the probability that an arbitrary subject responds in a specified way to a dose $x$. In this article we consider maximum likelihood estimation of $\Pi\left(x_{1}\right), \ldots, \Pi\left(x_{k}\right)$ subject to the constraint that II is sigmoid. Of particular interest is the nature of $\Pi$ at very small $x$. Upper confidence bounds for $\Pi(x), x \leqslant x_{1}$, are discussed. These confidence bounds depend on an inequality that holds when $\Pi$ is convex on $\left[0, x_{1}\right]$. A test of concavity against the alternative under sigmoidicity and related tests are discussed. Rejecting concavity requires evidence that II is convex on $\left[0, x_{1}\right]$.

- Research sponsored by U.S. DOE Office of Health and Environmental Research.

\subsection{5}

\section{EVERYDAY APPLICATION OF THE CELL MEANS MODEL}

\section{R. L. Schnoyer}

[Abstract of The American Statimician 38(1), 49 (1984)]

Statistical computer packages that routinely generate the functional form of estimable functions help the user to understand better the difference between the various similar hypotheses in an unbalanced analysis of variance. Another way to understand different hypotheser is to consider estimable functions expressed in terms of cell means. This article proposes routinely generating cell means estimable functions and gives a formula for translating estimable functions in a given parameterization to the cell means equivalent and vice versa. We discuss an example.

- Research sponsored by US. DOE OfTice of Health and Enviroamental Research.

\subsection{6}

\section{A DISTRIBUTION-FREE ANALYSIS FOR ACCELERATED TESTTNG AT SEVERAL LEVELS OF A SINGLE STRESS*}

\section{R. L Schwoyer}

[Abstract of Technometrics (in p.os)]

Consider the following experiment: At stress levels $x_{1}<\ldots<x_{k}, n_{1}, \ldots, n_{k}$ items are put on test. The failure times of those items failing before times $i_{1}^{*}, \ldots, l_{k}^{*}$ are recorded, and the remaining failure times are censored. Let $P(t, x)$ be the probability that an item, stressed at level $x$, fails on or before time $t$. We consider models of the form $P(t, x)=\Phi[g(t, x)]$, where $g$ is a real-valued function specified up to an unknown parameter $c$, and $\Phi$ is an unspecified continuous distribution function. Under these assumptions a Kolmogorov-Smirnov-type test is discussed as a means of estimating $c$ as well as $P\left(t_{0}, x_{0}\right)$ for specified $t_{0}$ typically greater than $t_{i}$ and $x_{0}$ typically less than $x_{1}$. If $n_{1}=\cdots=n_{k}$, the method extends to experiments which at each stress level are terminated after a srecified number of failures is observed. Extensions to models of higher dimension are also considered.

-Research sponsored by U.S. DOE Orfice of Health and Environmental Research. 


\section{A NONPARAMITTRIC ANALYSIS FOR STIJPP DOSE-RISPPONSE BIOASSAYS*}

\section{R. L. Schoyer J. J. Beanchan J. F. MeCarthy'}

(Abatract of paper presented at Eastern North American Regional Spring Meeting of the Biometric Society. Orlandh. FL, March (1-14, 1984)

In a bioassay experiment doses $x_{1} \ldots \ldots x_{k}$ of a test substance are administered to $n_{1} \ldots . . . n_{k}$ animal subjects. Let $p_{i}$ be the probability that a subject responds to drse $x_{i}$. In many experiments there is a nontrival possibility that the true dose response is so steep relative to the grid $x_{1} \ldots . . . x_{k}$ that the following hypothesis is tenable: there is a $j=0$.....or $k$ for which

$$
\begin{aligned}
0=\cdots & =p_{j-1} \leqslant p_{j} \leqslant 1 / 2 \\
\leqslant p_{j+1} & \leqslant p_{j+2}=\cdots=1
\end{aligned}
$$

where $p_{-1}=p_{0}=0$, and $p_{k+i}=$ $p_{k+2}=1$. If this hypothesis is tnje, the usual methods of statistical analysis (e.g., probit, logit, or Spearman-Karber) are incapable of producing valid confidence intervals for the LDSO (median lethal dose), which is the customary goal of bioassay. An exact likelihood ratio teat of the hypothesis against a general monotone alternative is discussed. A conservative confidence interval for the LDSO, consistent with the hypothesis, is also given.

\footnotetext{
-Recearch sponsored by U.S. DOE Orive or Heallh and Environmental Reacarch.

Environmental Sciences Division.
}

\section{DISTRIBUTIONS ARISING FROM REINFORCEMIENT-DLPLETION URN MODEL*}

\author{
L. R. Swento' $\quad$ K. O. Bownan
}

[Abatract of Scandinavian Jownal of Statistios (iv prea)]

For the reinforcement-depletion urn model involving balls of several colors, the multivariate factorial moment generating function (fmgf) can be described as a product of symbolic polynomial expressions operating on positive-value variables, which is a complicated procedure. We offer a much simpler recursive scheme which adumbrates convolution formulas; however, the scheme involves difference operators. The fmgf for the state of the um at any cycle turns out to be in the form of products of symbolic fmgf of urn problems.

\footnotetext{
- Research sponsored by Applied Mathematical Sciences Research Program, U.S. DOE Ofince of Energy Revearch.

'Advanoed Computational Methods Center, University of Georgia, Athens, GA.
}

8.109

\section{ON THE DISTRIBUTION OF \\ THE TIME REQUIRED TO \\ REMOVE WHITE BALLS \\ FROM AN URN*}

\author{
M. Solvel ${ }^{\prime}$ S. R. Bernant \\ V. R. R. Uppolari
}

(Abotract of paper presented at the Federation of the American Society for Experimental Biology, Anabeim, CA, April 21-26, 1984)

Balls are removed one at a time at equal time intervals from an urn initially containing $w_{0}$ white balls and a large number $b$ of black balls, and each black or white ball is immediately replaced by a black ball. The distribution of the number of white balls remaining after $l$ iterations (under certain limiting operations) is taken from the literature. The problem is to use this result 
to find the time required to remove a fixed number of white balls $w_{1}$ from the urn. We ithen find the mean and variance of this distribution and also look at the special case when $w_{1}=w_{0}$.

'Research sponsored by U.S. DOE OTroc of Health and Environmental Research.

University of California, Santa Barbara, CA.

FHeallh and Safety Research Division.

8.110

FOUR-URN CATENARY MODEL FOR EXCRETION*
M. Sobel'
S. R. Bermant
V. R. R. U Und
C. W. Nestor, Jr.

[Abstract of Bull. Makh. Biol. 46, 219 (1984)]

A chain-like arrangement of four urns (a catenary system) into which balls of two different colors (white, corresponding to radio atoms, and black, correspunding to stable atoms) are being transferred is used to simulate the transport of atoms down the GI tract of man and animals. Into the first urn (stomach) are placed $w_{0}$ white balls and $r$ black balls while in the 2 nd (small intestines) and 3rd (large intestines) urns, only $r$ blacks are put in, with no whites. A sample of size $r$ is transferred from the 1st, 2nd and 3rd urns to the 2nd, 3rd and 4th (infinite universe) urns. From the random variable difference equations, the first and second moments for the distribution of the number of radio atoms present in each urn are obtained. The variance of the contents of radio atoms in the excretion urn is

$$
w_{0}^{2} P\left(\frac{l}{\Delta l}\right)\left|1-P\left(\frac{l}{\Delta l}\right)\right|,
$$

where

$$
P\left(\frac{t}{\Delta t}\right)=e^{-(t / \Delta)}\left[1+\left(\frac{t}{\Delta t}\right)+1 / 2\left(\frac{l}{\Delta l}\right)^{2}\right] .
$$

In the equation, $t$ is the time after a single intake of $w_{0}$ radio atoms into the stomach, and $\Delta t$ is the mean residence time in the stomach. The model is tested with experimental data and a higher variance than the data is obtained.

- Research sponsored by U.S. DOE Offrce of Health and Environmental Research.

University of California, Santa Barbera, CA.

$t_{\text {Heahh and Safety Research Division. }}$

${ }^{5}$ Computing and Telecommunications Division.

8.111

\section{LIMITING VALUES FOR THE RBE OF FISSION NEUTRONS AT LOW DOSES FOR LIFE SHORTENING IN MICE*}

\section{J. B. Storer ${ }^{\dagger} \quad$ T. J. Mitctell}

[Abstract of Rediotion Research 97,396 (1984)]

We have analyzed recently published data on the effects of low doses of fission neutrons on the mean survival times of mice. The analysis for single-dose exposures was confined to doses of 20 rad or less, while for fractionated exposures only total doses of 80 rad or less were considered. We fitted the data to the frequently used power function model: life shortening $=\beta D^{\lambda}$, where $D$ is the radiation dose. We show that, at low doses per fraction, either (1) the effects are not additive or (2) the dose-effect curve for single exposures cannot show a greater negative curvature than about the 0.9 power of dose. Analysis of the data for $\gamma$ rays showed that an exponent of 1.0 gave an acceptable fit. Taken together, these findings indicate that the RBE for neutrons cannot change more rapidly with neutron dose than about $\mathrm{RBE}_{N} \approx k / D_{N}^{0.1}$. This conflicts with the more widely accepted relationship, $\mathrm{RBE}_{N} \approx k / D_{N}^{0.5}$. Because of the inherent implausibility of exponents $<1.0$ for the neutron dose-effect curves at low doses we conclude that at neutron doses of $20 \mathrm{rad}$ or less the RBE for life shortening is constant and ranges from 13 to 22 depending on mouse strain and sex.

\footnotetext{
- Research sponsored by U.S. DOE Office of Health and Environmental Research.

'Biology Divition.
} 
8.112

\section{AN EMPIRICAL STUDY ON THE STABILITISS OF DIFFERENCE ESTIMATORS*}

\author{
H. Tseot T. Wrigh
}

(Abaract of paper to be presented at the Anaval Joint Meeting of the American Stativical Aswociation, Imatitute of Mathematical Statistics Biometric Society, Las Vegas, NV, Auguse 5-8, 1985)

Stabilities oi difference estimators were computed for a number of joint sampling strategies which will maximize (or minimize) the number of expected overlaps in unequal probability sampling without replacement. The investigation considers difference estimators based on samples of size $n$ $=2$ on two sampling sccasions. These joint sampling strategier were generated under three natural populations and four component UPSwor sampling rules.

- Research sponsored by U.S. DOE Energy Information Administration and the OTice of Encrgy Research.

tData and Amalysis Section, Energy Division.

\subsection{3}

\section{AN EMPIRICAL STUDY ON MAXIMIZING (OR MINIMIZING) THE NUMBER OF RETENTIONS IN UNFQUAL PROBABILITY SAMPLING WITHOUT REPLACEMENT: TWO UNITS PER STRATUM*}

\section{H. Tsaot $\quad$ T. Wright}

(Abstract of paper oresented at the American Statistical Aswociation Annual Meeting. Philadelphia, PA, Auguar 14, 1984; Proc. Section on Survey Research Methods of the Amertcan Statistical Association, p. 218, 1984)

Sampling strategies are determined to maximize (or minimize) the expected number of overlaps between two successive samples using the linear programming approach. The abilities to control overlaps between two successive samples are compared for five methods of sampling with two units selected, in terms of the maximum (or minimum) expected number of overlaps achieved. Six different types of artificial populations are considered to support the empirical study and to illustrate the differences among the five sampling procedures.

- Research sponsored by U.S. DOE Enerty laformation Administration and the Office of Energy Research.

'Data and Analysis Section, Enery Division.

\subsection{4}

\section{NONPARAMETRIC ESTIMATION OF THE DISTRIBUTION OF TIME TO ONSET FOR SPECTFIC DISEASXS \\ IN SURVIVAL/SACRIFICE EXPLRIMENIS*}

\section{B. W. Torun't T. J. Mitchel \\ [C.batrat of Biometrics 4., 41 (1984)]}

This paper concerns the analysis of an animal survival/sacrifice experiment designed to investigate the incidence of a particular disease of interest. The disease is assumed to be irreversible and detectable only at death, for example by a necropsy. Each observation can be of one of three types: (1) death caused by the disease; (2) death from a competing cause such as sacrifice, with the disease present; or (3) death with the disease absent. A two-dimensional EM algorithm is proposed for the nonparametric maximum likelihood estimation of the distributions of the time to onset and of the time to death from the disease. These can be compared with nonparametric estimators recently proposed by Kodell, Shaw and Johnson (1982, Biometrics 38, 43-58) and by Dinse and Lagakos (1982, Biometrics 38, 921-932). A slight modification of the algorithm permits the construction of likelihoud-based interval estimates of quantiles of the distributions. Some extensions and generalizations are indicated.

- Reacarch sponsored by U.S. DOE Office of Healtb and Environmental Research.

'School of Operations Rexearch and Indussrial Enginecring. Cornell University, Ithaca, NY. 
8.115

\section{METHODS IN RISK MANAGEMENT - A WORKING PAPER*}

\section{R. R. Uppaluri}

(Abstract of paper presented at First United States-Japa. Workshop on Risk Management, Tsukuba, Japan, October 28-31, 1984)

Methods in risk analysis to detsrmine the health and environmental effects oi hazards to individuals and society are evolving in this rapidiy developir:g ineld. In this paper. we briefly discuss the current distinctions between risk assessment and risk management and discuss snme methodological issues. We conclude this paper with a method useful for prioritization in connection with the environmental, health, and safety deficiencies at a number of sites.

-Reseirch sponsmed by U.S. N'iclear Regulatory Commission.

8.116

\section{THE DISTRIBITTON OF THE FIRST j DIGITS OF BETA RELATED RANDOM VARIABLES*}
V. R. R. Uppuluri
S. A. Patil ${ }^{\dagger}$

(Abstract of Commun. Sialist.-Simula. Computa. 14(2), 467 (1985)]

This paper gives the discrete distribution of t'. e first $j$ significant digits of two r.andom variables: (1) a beta variable with integer parameters $m$ and $n$, and (2) the reciprocal of (1). As a special case fo: $n=1$, we obtain the distribution of the first $j$ significant digits of the powers of uniformly distributed random variables. These generalize the results of Kennard and Reith (1981) and Friedterg (1984), who considered only uniformly distributed random variables.

- Research sponsored by Oak Ridge National Laboratory.

${ }^{\dagger}$ Mathematics Department, Tennessee Technological University, Cookeville, TN.
8.117

\section{STATISTICAL METHODS AND THE MPROVEMENT OF DATA QUALTYY}

\section{T. Wright, Editor}

[Abstract of a book, Academic Press, New York, NY (1983)]

This volume is the proceedings of the Small Conference on the Improvement of the Quaity of Data Collected by Data Collection Systems, Oak Ridge, TN, November 11-1982.

- Research sponsored by Applied Mathematical S: Research Program, U.S. DOE OITice of Energy Kesearch.

\subsection{8}

\section{SOME USEFUL NOTES ON SIMPLE RANDOM SAMPLING*}

$$
\text { T. Wright H. J. Tseo }{ }^{\dagger}
$$

[Abstract of J. Quality Tech. 17(2), 67 (1985)]

Some useful resu'ts about simple ranniom samples are given which are not generally known or are often assumed to be true but with some uncertainty $b$ :s the experimenter. Such results, when called to the attention of users of sampling techniques, should lead to a better understanding of many of the results that are built on the concept of a simple sandom sample.

\footnotetext{
- Research sponsored by Applied Mathematical Scienies Research Program, U.S. DOE Office of Energy Research.

'Data and Analysis Section, Energy Division.
} 
8.119

\section{ON AN OPTTMAL SOLUTTON FOR \\ MAXIMIZING THE PROBABILTYY \\ OF RETENTION IN PPS SAMPLING*}

\section{T. Wright H. J. Tseot}

[Abstract of Linear Algebra and Its Applications 4, 67 (1985); also abstract of paper entitled On Maximizing the Probability of Retention in PPS Sampling." presented to the Statistics Department University of Tenecsece, Kroxville, TN, March 7, 1984]

A direct alternative proof of Keyfitz's optimal solution to the problem of maximizing the probability of retention in sampling on a second occasion is given, using techniques of elementary linear algebra. The proof and comments help one to better understand Keyfitz's solution, and they clearly demonstrate that ti. : ilosed form solution of Keyfitz is one of a possible infinity of solutions offered by a linear programming approach. We also give one of those other solutions offered by linear programming, which is easy to obtain by hand calculations using only the operation of subtraction.

- Research sponsored by U.S. DOE OTice of Energy Research and the Energy Information Administration.

'Data and Analysis Section, Energy Division. disease death or cerebrovascular disease deaths to levels of 19 inorganic parameters in drinking water. The study included 505 cases and 854 controls, all of whom obtained their drinking water from a individual nonchlorinated well on the farm. Drinking water of cases was significantly softer than that of controls. Differences between the two groups were limited to the range of total hardness below $200 \mathrm{mg} / \mathrm{L}$. The relationships were as strong or stronger when respondents reporting artificial softening were removed. A variety of potential confounders of the relationship were examined, and none were found to eAplain the difference in hardness in the two groups. Drinking water of cases also had ignificantly lower carbonate hardness (alkalinity) Of the metals, cadmium, lead, and zine levels vere significantly higher for cases. Levels of iron, copper, manganese, nitrate, sodium, and fluoride were not different in the two groups. The levels of cadmium and lead in the water were extremely low, and the significant difference between the groups was limited to the percentage of samples with values above analytic detection limits.

- Research sponsored by U.S. DOE OITice of Health and Environmental Rewearch.

${ }^{\prime}$ Health and Safety Research Division.

FWisconsin State Laboratory of Hygienc. University of Wisconsin, Madison, WI.

\section{DRINKING WATER INORGANIC, AND CARDIOVASCULAR DISFASE: A CASE-CONTROL STUDY AMONG WISCONSIN FARMERS*}

\author{
E. A. Zeighamit \\ E. E. Callet \\ M. D. Morris \\ P. S. MeSweenyt
}

B. A. Schuknechtt

(Abstract of paper presented at the Conference on Inorganica
in Drinking Water and Cardiovaccular Disease, Univerity of
Massachusets, Amherst, MA, May I-3, 1984)
A case-control study of Wisconsin farmers has
examined the relationship of coronary artery 
APPENDICES

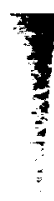




\title{
SCIENTIFIC AND PROFESSIONAL ACTIVITIES
}

\author{
January 1, 1984 - June 30, 1985
}

L. S. Abbott

Editor

- The Acorn, newsletter published by Oak Ridge/Knoxvill. Section of the American Nuclear Society.

Associate Editor

- Risik Newsletter, published by Society for Risk Analysis.

\section{Alexiades}

\section{Invited lectures}

- "Solidification of $\mathrm{Hg}$-Cd-Te Alloy," Colloquium, NASA, Space Science Lab., Marshall Space Flight Center, Huntsville, AL, April 1985.

- The ORNL Binary Alloy Solidification Model," Workshop on Alloy Solidification, Florence, Italy, May 1985.

- "Minimizers of the Free Energy in the Thermodynamic Theory of Fluid Interfaces," Colloquium, Inst. "J. Louis Lagrange," Univ. di Torino, Turin, Italy, May 1985.

- "Remarks and Questions about Hyperbolic Stefan Problems," Colloquium, Inst. "J. Louis Lagrange," Univ. di Torino, Turin, Italy, May 1985.

- "Minimizers of the Free Energy in the Thermodynamic Theory of Fluid Interfaces," Colloquium, Math. Dept., Univ. of Crete, Iraklion, Greece, May 1985.

- "Alloy Solidification with Constitutional Supercooling," Colloquium, Physics Dept., Univ. of Thessaloniki, Greece, May 1985.

ORivL Instructor

- Useful Mathematical Methods for Scientists and Engineers, March - June, 1985.

\section{Aseciate Profeseor}

- Mathematics Department, University of Tennessee, Knoxville, TN.

\section{Award}

-. UTK-ORNL Scienc. Alliance Research Incentive Award, 1985.

\section{Reviewer}

Mathematics Reviews

\section{Referee}

Applicable Analysis 
- SIAM Journal of Mathematical Analysis

- Nonlinear Analysis

- International Journal of Heat and Mass Transfer

- ASME Journal of Heal Transfer

R. G. Alsmiller, Jr.

Member

-- National Committee on Radiological Protection, Scientific Committee 52 on Conceptual Basis of the Calculations of Dose Distributions.

\section{Chairman}

- Department of Energy Advisory Panel on Accelerator Radiation Safety for the Review of TEVATRON I and TEVATRON II at the Fermi National Accelerator Laboratory.

- Department of Energy Advisory Panel on Accelerator Radiation Safety for the Review of the Stanford Linear Collider at the Stanford Linear Accelerator Center.

Invited paper

- Spacecraft Shielding: The Contribution to the Absorbed Dose and Dose Equivalent from Nuclear Reaction Products," Workshop on Space Radiations sponsored by the National Counsil on Radiation Protection and Mtasurements. Washington, D.C., January 14-16, ;985.

Referee

- Nuclear Science and Engineering

- Nuclear Technology/Fusion

-- Radiation Research

- Physics in Medicine and Biology

- Neutron Dosimetry in Radiation Protection (Book)

- Nuclear Instruments and Methods

C. M. Anthony

Deputy Representative

- DOE National Energy Software Center (NESC).

\section{J. Barhen}

Member

- Program Committee, IEEE Second Annual Conference on Artificial Intelligence Applications, Miami Beach, FL, December II-13, 1985.

\section{Chairman aad Organizer}

-. Sessions on Advances in Concurrent Computation, 1985 International Computers in Engineering Conference, Boston, MA, August 4-8, 1985.

\section{Moderator and Organizer}

Panel on Future Directions in Concurrent Comoutation, 1985 International Computers in Engineering Conference, Boston, MA. 


\section{Geest Editor}

- CIME (Springer Verlag), Special Issue devoted to Advances in Multiprocessing (Winter 1985).

Invited papers

- "Hypercube Concurrent Computation and Virtual Time for Robotics Applications," IEEE Workshop on Special Computer Architectures for Roboties and Automation, St. Louis, MO, March 1985.

-- An Intelligent Machine Operating System for a Hypercube Ensemble Architecture," DOE Workshop on Planning and Sensing for Autonomous Navigation, Los Angeles, CA, August 1985.

Invited talk

- "Mapping Irregular Problems on a Concurrent Computation Ensemble" (with J. R. Einstein), Conference on Hypercube Multiprocessors, Knorville, TN, August 1985.

\section{J. L. Bartley}

Invited paper

- VITAMIN-E 174n, 38g Cross-Section Library for Fusion Neutronics Calculations" (with R. W. Roussin and J. E. White), presented at ANS Meeting, New Orleans, LA, June 1984.

\section{J. J. Beauchamp}

Represeutative

- ORNL Professional Education Resource Committee.

Instructor

- ORNL In-Hours Continuing Education Program.

Referee

- Technometrics

- Journal of Statistical Computation and Simulation.

\section{K. O. Bowman}

\section{Member}

- National Science Foundation Advisory Committee on Equal Opportunities in Science and Technology.

- National Science Foundation Reviewing Committee on VPW.

- American Association for the Advancement of Science Committee on Section T Information, Computing, and Communication.

- American Statistical Association Committee on Representative to AAAS.

- COPSS, Committee on Representative to AAAS.

- Program Committec, SIAM Symposium on Frontiers in Computational Statistics.

- AAAS National Network of Minority Women in Science, Advisory Panel.

-.. International Editorial Board, Communications in Statistics

\section{Chairman}

Coordinating Committee on Equal Opportunities in Statistics, American Statistical Association. 


\section{Lecturer}

- Visiting Lecture Program, Committee of President of Statistical Societies (COPSS).

\section{Co-Organizer}

- Symposium, "Journeys into digher Dimensions: Graphics in Mathematics, Statistics, and Perception," AAAS Annual Meeting, New York, 1984.

Associate editor

- Journal of Statistical Computation and Simulziton.

Contributing eliter

- Current Index to Statistics.

\section{Reviewer}

- Science, American Association for the Advancement of Science.

- Research Proposal, National Science Foundation.

\section{Invited talles}

- The Estimation Problem of Poisson-Poisson Distribution and Its Application." Presented at Taipei, Taiwan, September 20, 1984; the 12th International Biometric Conference, Tokyo, Japan, September 2-8, 1984: the Institute of Statistical Mathematics, Tokyu, Japan, September 28, 1984; Keio University, Yokohama, Japan, October 4, 1984; Tukuba University. Tukuba, Japan, October 9, 1984; Chinese University of Hong Kong, Hong Kong, October 15, 1984; and Tokyo Science University, Tokyo, Japan, October 18, 1984.

- The Distribution of Estimates with Special Reference to Those Involving Sample Moments." Presented at Union - $f$ Japanese Scientists and Engineers, Tokyo, Japan, May 16, 1985; Chiha University, Chiha, Japan, May 31, 1985; and Tokyo University, Tokyo, Japan, June 12, 1985.

- "Approximations to Distributions," Wright State University, Dayton, Ohio, March $29,1985$.

\section{Referee}

- Annals of Statistical Mathematics

-- Communications in Statistics

-- Journal of Statistical Computation and Simulation

\section{T. J. Burns}

Member

- Martin Marietta Energy Systems Inventor's Forum.

- Program Committee, American Nuclear Society Topical Meeting on Anticipated and Abnormal Plant Transients in Light Water Reactors, Jackson, WY, 1983.

\section{Special ascignnent}

To Economic Assessment Team supporting U.S. Department of Energy's Process Evaluation Board, April 1984 - June 1985.

\section{G. Cacuci}

Member

- Department of Ene:gy Working Group on Reassessment of A-Bomb Dosimetry. 
- ORN!. Proposal Review Committee.

- Ph.D. Committee, Departm: 1 of Nuclear Engineering, University of Tennessee, 1984.

\section{Secretary}

- National Planning Committee, American Nuclear Society.

\section{Associate Elitor}

- Nuclear Science and Engineering.

Profeseor (Part-tine)

- Departmest of Nuclear Engineering, University of Tennessec.

\section{Exteral Examiner}

- Ph.D. Committee, McMaster University School of Graduate Studies, Ontario, Canada, 1985.

Who's Who in Frontier Science and Technology, First and Sucond Editions.

Who's Who in the World, Seventh Edition.

The International Who's Who of Contemporary Achievement, 1985 Edition.

\section{Iovited papers}

- Sensitivity/Uncertainty Analysis of Radiation Transport in the Hiroshima AirOver-Ground Environment" (with J. V. Pace III), California Institute of Techrology, February 1984.

- Sensitivity Analysis of the Oregon State University Atmospheric General Circulation Mode! by the Adjoint Method" (with M. C. G. Hall), Lawrence Livermore National Laboratory, April 1984.

- "Dose Reassessment Sensitivity/Uncertainty Analysis: Illustrative Application to Radiation Transport in the Hiroshima Air-Over-Ground Environment," National Academy of Sciences, Washington, D.C., May 1984.

- "Sensitivity/Uncertainty Analysis for the A-Bomb Dose Reassessment Program," U.S.-Japan Joint Workshop for Reassessment of A-Bomb Radiation Dosimetry in Hiroshima and Nagasaki," California Institute of Technology, February 1985.

Invited seminar

- "Global Sensitivity Analysis of Nonlinear Systems," Oregon State University, Corvallis, Oregon, April 1984.

Invited colloquium

- "Efficient Sensitivity Analysis of Large Nonlinear Systems with Feedback," Coiumbia University, October 1984.

Referee

- Physical Review Letters

- Journal of Mathematical Physics

Journal of Computational Physics

\section{R. J. Carter}

\section{Invited papers}

"ORNL Training/Test Research," Third Electric Power Research Institute Adyisory Committee Meeting on Simulator Evaluation, December 12, 1984.

"ORNL Training Research," Department of Energy Reactor Training Information Exchange Meeting, June 25 27, 1985. 
- Nuclear Power Plant Simulation Facility Evaluation Methodology," (with P. M. Haas), Third Institute of Electrical and Electronics Engineers Conference on Human Factors and Power Plants, June 23-27, 1985.

\section{S. N. Cramer}

\section{Techaical Coordimator}

- RSIC Workshop on the MORSE Monte Carlo Code, Oak Ridge, TN, June 17-21, 1985.

\section{Invited paper}

- "Monte Carlo Techniques for Analyzing Deep Penetration Problems," International Meeting on Advances in Nuclear Engineering Computation Methods, Knoxville, TN, April 9-11, 1985.

\section{Referee}

\section{- Nuclear Science and Engineering}

\section{G. de Saussure}

Member

- Program Committee and Session Organizer, Topical Meeting on Reactor Physics and Shielding, American Nuclear Society, September 17-19, 1984.

- General Program Advisory Committee, Internationa: Conference on Nuclear Data for Basic and Applied Sciences, May 13-17, 1985.

- Technical Program Committee, Fifth Pacific Basin Nuclear Conference, American Nuclear Society, May 1985.

- 1985 Program Committee, Conference on Artificial Intelligence Applications, IEEE Computer Society, August 1985.

\section{Honorary Professor}

- Nuclear Engineering Department, University of Tennessee.

\section{Referee}

- Nuclear Science and Engineering

- The Physical Review

\section{J. K. Dickens}

Member

- American Nuclear Standards Working Group 5.1, Decay Heat Standard.

-- American Nuclear Standards Working Group 5.2, Fission Product Yields.

\section{Correspondent}

- Designated ly IAFA Working Group on Fission Prnduct Nuclear Data as correspondent on worldwide experimental measurements of fission product decay heat (1977 continuing).

\section{Invited lecture}

"Nuclear Safety and the Future of Nuclear Power," Denison University, Granville, Ohio, May 1985. 


\section{Referee}

- Nuclear Science and Engineering

- The Physical Review

\section{F. C. Difilippo}

Invited lect res

- Noise Techniques and the Sirveillance of Nuclear Power Plants," AEC Headquarters (Buenos Aires, Argentina) and Balseiro Institute (Bariloche, Rio Negro, Argentina), June 1985.

- "Design of High Intense Steady Neutron Sources," AEC Headquarters (Buenos Aires, Argentina), and Balseiro Institute (Bariloche, Rio Negro, Argentina), June 1985.

\section{Referee}

- Nuclear Science and Engineering

\section{J. B. Drake}

Member

- ORNL Computer User Advisory Committee

\section{J. R. Einstein}

lavited talt

- "Mapping Irregular Problems on a Concurrent Computation Ensemble" (with J. Barhen), Conference on Hypercube Multiprocessors, Knoxville, TN, August 1985.

W. W. Engle, Jr.

Invited payser

- "Comparison of Candidate Shielding Materials for Space Power Reactor Applications," American Nuclear Sosiety Annual Meeting, New Orleans, June 1984.

\section{Award}

- Martin Marietta Energy Systems Technical Achievement Award, 1985.

\section{Chairman}

- Ariaingements Committee, International Meeting on Advances in Nuclear Engineering Computational Methods, Knoxville, TN, April 9-11, 1985.

\section{Member}

- Technical Program Committee, International Mieeting on Advance in Nuclear Engineering Computational Methods, Knoxville, TN, April 9-11, 1985.

\section{G. F. Flanagan}

\section{Member}

- Standards Committec 5.4, Institute of Electrical and Electronics Engineers (IEEE).

-.- Standards Committee 5.1, Institute of Electrical and Electronics Engineers (IEEE).

- Standards Committee 19.3, American Nuclear Society. 
- Editorial Board, Risk Analysis Journal.

-- Ph.D. Recruiting Program. ORNL.

- ORNL Speaker's Bureau.

- American Nuclear Society Speaker's Bureau.

Crainen

- International Topical Meeting on Fast Reactor Safety, Knoxville, TN, April 21-25, 1985.

Editor

- Kisk Newsletter, published by Society for Risk Analysis.

Representative

- ORNL Advisory Committee for Office of Risk Analysis.

Invited paper

- Nuclear Safety and Risk Analysis," East Kentucky State University, Richmond. KY, December 1984.

Instructor

- ORAU Faculty Institute, "Risk and Nuclear Safety Studies - Critique," Oak Ridge, TN, June 1985.

Referee

- Nuclear Safety Journal

- Nuclear Technology

- Risk Analysis Journal

\section{E. L. Frome}

\section{Member}

- ORAU/University of North Carolina Research Planning Group for DOE Health and Mortality Studies.

\section{Chairman}

- American Statistical Association Conference on Radiation and Health (1984, 1985).

Associate editor

- The American Statistician, 1983-present.

Invited talk

- "Regression Methods for Binomial and Poisson Distributed Data," American Association of Physicists in Medicine Symposium on Regression Methods in Health Sciences, Mobile, AL, March 1984.

Referee

- Journal of the American Statistical Association

- Communications in Statistics

C. Y. Fu

Member

- Evaluation Methods Subcommittee, Cross Section Evaluation Working Group (CSEWG).

- Standards Subcommittee, Cross Section Evaluation Working Group (CSEWG). 


\section{Invited talk}

- "Applicaticas if Nuclear Models to Neutron-Nuclear Cross-Section Calculations," International Symposium on Fast Neutrons in Science and Technology in combination with the Ind IAEA Coordinated Research Programme Meeting, University of Chiang Mai, Thailand, February 4-8, 1985.

Referee

- Nuclear Science and Engineering

\section{R. E. Funderlic}

Menber

- Organization Committee for Conference on Hypercube Multiprocessors, August 1985.

\section{Invited talks}

- The Hypercube Architecture and Some Aspects of Data Flow for Parallel Computation of Missized Matrix Problems" (with G. A. Geist), Parallel Computing Seminar, Mathematics Dept., North Carolina State University, April 10, 1985.

- "Updating LU Factorizations for Computing Stationary Distributions" (with R. J. Plemmons), Gatlinburg-9 International Conference on Numerical Algebra, Waterloo, Ontario, July II, 1984.

\section{Reviewer}

- American Mathematical Society Mathematical Reviewers.

\section{Referee}

- BIT

- SIAM Joumal Scientific and Statistical Computing

\section{Editorial Board}

- SIAM Journal Algebraic and Discrete Meshods

\section{Assistant Professor}

- Departments of Mathematics and Computer Science, University of Tennessee, Knoxville, TN (part time).

\section{T. A. Gabriel}

Member

- ASTM Subcomrnittee E10.08, Procedures for Neutron Radiation Damage Simulation.

- ASTM Subcomnittee E10.08.02, Simulation of Helium Effects in Irradiated Spectral Tailoring.

\section{Invited papers}

- "An Introductior to Combinatorial Geometry" (with M. B. Emmett), CERN Workshop on Shower Simulation for LEP Experiments, Geneva, Switzerland, Janu. ary 29-31, 1985.

- The High Energy Hadronic Transport Code, HETC," CERN Workshop on Shower Simulation for LI:P Experiments, Geneva, Switzerland, January 29-31, 1985.

Award

Outstanding Service Award for Professional Excellence from the Radiation Protection and Shieldin,; Division of the American Nuclear Society, June 10, 1985. 


\section{Referee}

- Nuclear Science and Engineering

- Nuclear Instruments and Methods

- Nuclear Technology/Fusion

\section{G. A. Geist}

Meaber

- Organization Committee for Conference on Hypercube Multiprocessors, August 1985.

Invited balks

- "Heat Transfer Considerations in Passive Solar with Energy Storage" (with W. 0. Soloman), Tel Aviv University, June 18, 1984.

- Modeling of Bulk Nucleation and Multiple Phase Front Formation in Pulsed Laser Melted Amorphous Silicon" (with R. F. Wood et al.), Materials Research Society Symposium, Boston, MA, November 26-30, 1984.

- The Hypercube Architecture and Some Aspects of Data Flow for Parallel Computation of Missized Matrix Problems," (with R. E. Funderlic), Paralkel Computing Seminar, Mathematics Department, North Carolina State University, April 10, 1985.

\section{G. Gosslee}

Member

- Advisory Committee to the Department of Statistics, Ohio State University.

\section{J. Gray}

\section{Invited talks}

- "Self-Consistent Approximations !or Random Alloys," ORAU Traveling Lecture, Williamsburg, VA April 27, 1984.

- "A Fractal Model for Charge Diffusion Across a Rough Interface," NATO Advanced Study Institute on "Scaling Phenomena in Disordered Systems," Geilo, Norway, April 8-19, 1985.

\section{R. Gwin}

Award

- Martin Marietta Publications Award, 1985

\section{M. Haaland}

Advisor

- National Council on Radiation Protection and Measurements, Scientific Committee 63: Radiation Exposure Control in a Nuclear Emergency.

\section{Evaluator}

- Pilot Course for Radiological Defense Officers, National Emergency Training Center, Emergency Management Institute, Emittsiurg, MD, January 23-27, 1984. 


\section{Inrited paper}

- Should We Protect Ourselves from Nuclear Weapon Effects?;" The American Physical Society, Detroit, Ml, March 1984.

Invited thls

- Radiation Exposure Control ior Emergency Workers," Meeting of Radiological Defense Officers, Kansas City, MO, March 1984.

- "Lower Atmospheric Chemistry and Stratospheric Ozone," Annual Meeting of Radiołogical Defense Officers, Kansas City, MO, March 1984.

- "In the Shadow of Ground Zero," Annual Meeting of The American Civil Defense Association, Daytona Beach, FL, November 1984.

- "A Review of the Nuclear Winter Scenario," sponsored by the Society for Risk Analysis for the WATTec Conference, Knoxville, TN, February 1985.

- Nuclear Winter," Annual Convocation of the University of Southern Maine, Fortland, ME, April 1985.

- "A Review of the Nuclear W'inter Soenario," Annual Meeting of the State of Maine College Physics Teachers, Porttand, ME, April 1985.

- "In the Shadow of Ground Zero," Annual Meeting of the Mobile County Emergency Management Agency, Mobile, AL, May 1985.

Referee

- Journal of Civil Defense

\section{P. M. Haas}

Member

- International Evaluation of Operational Practices Group (formed by EPRI).

- Professional Development and Accreditation Committee, ANS.

- Institute of Electrical and Electronics Engineers (IEEE), Nuclear Power Engineering Committee (NPEC) Subcommittee SC-7: Human Factors and Control Facilities.

- (Liaison Member) IEEE NPEC Subcommittoe, SC-5: Reliability.

- In-Plant Reliability Data System (IPRDS) Steering Committee.

\section{Chairman}

- Technical Program Committce, ANS/ENS International Topical "Adrances in Human Factors in Nuclear Power Systems."

- Centralized Reliability Data Organization (CREDO) Steering Committee.

- Invited Paper Session, Fastern Simulation Conference, Norfolk, VA, March 1985.

\section{Techaical Advisor}

- American Nuclear Society ANS 3.5 Commitiee on Simulators.

\section{Editor}

- Accident Analysis Section, Nuclear Sofety.

\section{Iovited papers}

- Simulator Experiments: Effects of NPP Operator Experience on Performance" (with L. H. Gray et al.), 12th Water Reactor Safety Research Information Meeting, Washington, DC, October 1984. 
- Training Related Rescarch and Development Conducted at ORNL for the U.S. NRC," IEEE Third Conference on Human Factors and Power Plants, Monterey, CA, June 1985.

- "Nuclear Power Plant Simulation Facility Evaluatior Methodology," IEEE Third Conference on Human Factors and Power Plants, Monterey, CA, June 1985.

- "Collection and Analysis of Training Simulation Data," IEEE Third Conference on Human Factors and Power Plants, Monterey, CA, June 1985.

- "Human Factors Research at ORNL," Meeting of International Evaluation of Operational Practices Group, Paris, France, November 1984.

-. "Human Factors Research at ORNL," Industrial Engineering Department, University of Tennessee, February 1985.

Invited panelist

- Panel Session, Training Effectiveness," Eastern Simulation Conference, Norfolk, VA, March 1985.

- 12th Water Reactor Safety Information Mecting, Washington, DC, October 1984.

- IEEE Third Conference on Human Factors and Power Plants, Monterey, CA, October 1984.

M. C. G. Hall

Iavited papers

- "Carbon Dioxide and Climate: Potential Impacts," 12th Annual WATTec Conference, Knoxville, TN, February 12, 1985.

- Sensitivity Analysis of the OSU Atmospheric General Circulation Model by the Adjoint Method," Lawrence Livermore National Laboratory, Livermore, CA, April 10, 1984, and Oregon State University, Covallis, OR, April 12, 1984.

- The Potential Climatic Effects of Increasing Carbon Dioxide," DOE Carbon Dioxide State-of-the-Art Report No. 3.

Referee

- Journal of the Atmospheric Sciences

\section{J. A. Harvey}

Member

- ORNL Accelerators and Radiation Sources Review Committee.

- Executive Committec, Division of Nuclear Physics, American Physical Society.

- Executive Committee, Electromagnetic Isotope Enrichmer.t Facility Users Group.

Labor Coordinator

- Engineering Physics and Mathematics Division.

\section{Secretary-Treasurer}

- Division of Nuclear Physics, American Physical Society, 1967-1985.

\section{Referee}

- Physical Review $C$ and Physical Review Letters

- Nuclear Science and Engineering 


\section{N. A. Hatmaker}

\section{Chirmen}

- (Registration) - MORSE-CGA Workshop, Holiday Inn, Oak Ridge, TN, June 17-20, 1985.

\section{T. Heath}

\section{Chairman}

- Organizing Committee, Conference on Hypercube Multiprocessors.

\section{Member \\ - Board of Directors, ACM-SIGNUM}

\section{Invited papers}

- "Computing a Sparse Basis for the Null Space of a Sparse Matrix," Gatlinburg-9 International Conference on Numerical Algebra, Waterloo, Ontario, Canada, July 1984.

- "Sparse Matrix Computations," 23rd IEEE Conference on Decision and Control, Las Vegas, NV, December 1984.

\section{Referee}

- Linear Algebra and Its Applications

- SIAM Journal Scientific and Statistical Computing

- IMA J. Numer. Anal.

- Mathematical Programming

- Algorithmica

- Parallel Computing

Proposal reviewer

- AFOSR

- NSF

\section{H. F. Hendrickson}

\section{Afirmative Action Representative}

- Engineering Physics and Mathematics Division.

\section{Coordinator}

- For summer students for EPIC.

\section{T. Ingersoll}

\section{Member}

- Shielding Data Testing Subcommittee, Cross-Section Evaluation Working Group (CSEWG).

- Executive Committee, Radiation Protection and Shielding Division, American Nuclear Society. 


\section{Chairman}

- Program Committee, Radiation Protection and Shielding Division, American Nuclear Society.

- U.S.-JASPER Shielding Program Working Group.

\section{Invited papers}

- Oak Ridge National Laboratory Tower Shielding Facility," JASPER Working Group Meeting, Tokyo, Japan, May 7-9, 1985.

- "Description of Proposed JASPER Work Scope," JASPER Working Group Meeting, Tokyo, Japan, May 7-9, 1985.

\section{Referee}

- Nuclear Technology

\section{C. Jorgensen}

\section{Mewber}

- National Academy of Sciences Special Panel on Human Operator Modeling (1985).

- Emerging Technologies Advisory Committec, National Security Industrial Association (NSIA).

- CAlA Program Review Committee (1985).

\section{Chairman}

- Human Factors Technical Advisory Group on Modeling, Department of Defense (1984-1985).

\section{B. L. Kirk}

Member

- American Nuclear Suciety Computational Benchmark Problems Committee.

- Society for Industrial and Applied Math Special Interest Activity Group on Supercomputing.

\section{H. E. Knee}

Member

-- Centralized Reliability Data Organization (CREDO) Steering Committee.

- CREDO Data Evaluation Working Group and CREDO Input/Output Working Group.

-.- In-plant Reliability Data System (IPRDA) Steering Committee.

- IPRDA Executive Working Group and Data Analysis Working Group.

\section{Chairman}

Publications Committee of the International Topical Meeting on Advances in Human Factors in Nuclear Power Systems, April 20-23, 1985.

\section{P. A. Krois}

\section{Invited paper}

"Collection and Analysis of Training Simulator Data" (with P. M. Haas), IEEE Third Conference on Human Factors and Power Plants, Monterey, California, June $2327,1985$. 
Mealuer

- Task Force on Human Factors Guidelines and Standards for Process Control, Human Factors Society.

Referee

- Journal of Risk Analysis

D. C. Larson

Meaber

- Evaluation Methods Subcommittec, CSEWG.

- Department of Erergy Nuclear Data Committee.

- LMFBR Nuclear Data Evaluation Task Force.

- Office of Basic Energy Sciences (OBES) Committee on Nuclear Data for Fusion Reactor Applications.

\section{Co-Cunirman}

- Engineering Physics and Mathematics Division seminars.

- General Purpose Evaluations Subcommittee, Cross Section Evaluation Working G̈roup (CSEWG).

Proposel reviewer

- Office of Basic Energy Sciences, Department of Energy, Low Energy Nuclear Physics.

\section{Invited paper}

- "High-Resolution Structural Material $(n, x \gamma)$ Production Cross Sections for $0.2<$ $E_{n} \leqslant 40 \mathrm{MeV}$," Conference on Nuclear Data for Basic and Applied Science, Sante Fe, NM, May 13-17, 1985.

Referee

- Nuclear Science and Engineering

R. A. Lillie

Member

- ASTM Subcommittee El0.08: Procedures for Neutron Radiation Damage Simulation.

Chairmasa

- ORNL Reactor Experiments Review Committee.

- Periodic ORR and BSR Experiments Review Subcommittee.

- ASTM Subcommittee E10.08.02: Simulation of Helium Effects in Irradiated Spectral Tailoring.

Lecturer

- "Discrete Ordinates Methods and Applications," Tennessee Industries Week, University of Tennessee, September 10-14, 1984.

Referee

- Nuclear Technology

- Nuclear Technology/Fusion 
- Journal of Fusion Energy

- Nuclear Science and Engineering

- Nuclear Instruments and Methods

\section{R. L. Macklin}

Menber

- Engineering Physics and Mathematics Division QA Audit Team.

- Engineering Physies and Mathematies Division Safety Inspection Quarterly Review.

Fellow

- American Association for the Advancement of Science.

- American Physical Society.

Invited lecture

- "Clues to the History of the Galaxy," Lucas Heights Research Laboratories, Lucas Heights, Australia, October 30, 1984.

Referee

- Nuclear Science and Engineering

- Physical Review

- Nuclear Physics

R. E. Maerker

Member

- ASTM E10.05 Subcommittec, 1978-present.

- Silielding Subcommittee, Cross Section Evaluation Working Group (CSEWG).

- Data Testing Subcommittee, Cross Section Evaluation Working Group (CSEWG).

Invited paper

- "Recent Progress and Developments in LWR-PV Calculational Methodology," Fifth ASTM-EURATOM Symposium on Reactor Dosimetry, Geesthacht, FRG, September 24-28, 1984.

Referee

- Nuclear Science and Engineering

- Journal of Nuclear Materials, Amsterdam, Holland

\section{F. C. Maienschein}

Couirman

- ORNL Cor-'sting Steering Committee (OCSC).

\section{Member}

- Nuclear Energy Agency Committee on Reactor Physics (NEACRP).

- ORNL Awards Advisory Committc.

- ORNL Affirmative Action Advisory Committee.

- ORNL Telecommunications Comrittee.

- American Nuclear Society Finance Committee. 


\section{J. March-Leuba}

Award

- Mark Mills Award, American Nuclear Society.

\section{B. F. Maskewitz}

Chiman

- American Nuclear Society Planning Committee (1983-1985).

Vice President/Presideat Elect Candidate

- American Nuclear Society, 1984 Election.

\section{Menber}

- American Nuclear Society Planning Committee (1979-1985).

- ANS-10 Subcommittee of the ANS Standards Committee (1965-1985).

- Ethies Committee of the Engineering Affairs Council of the American Association of Engineering Societies (1984-1985).

- Public Policy Committee, American Nuclear Society (1984-1985).

- ORNL Speakers' Bureau.

- ORNL Honors and Awards Advisory Committee.

- Organizing Committee, Symposium on Numerical Data for Energy Systems, Committee on Data for Science and Technology (CODATA) of the International Council of Scie.tific Unions (ICSU) Ninth Internationa! CODATA Conference, Jerusalem, Isri-1, June 24-28, 1984.

\section{Invited seminar}

- RSIC and International Technical Information Exchange," CEA/CEN Saclay, Gif-sur-Yvette, France (June 17-18, 1984) and ENEA Energy Research Center, Bologna, Italy (June 21-22, 1984).

Invited poster presentation

- "RSIC - A Technology Resource for Space Nuclear Power Systems," 1 st Symposium on Space Nuclear Power Systems, Albuquerque, New Mexico, January 10-13, 1984.

Invited paper

- "Overview of the Role of Data for Energy Systems," Ninth International CODA Conference, Jerusalem, Israel, June 24-28, 1984.

\section{Energy Systems Representative}

- DOE National Energy Software Center (1984-1985).

\section{Referee}

- Computer Code Abstracts, Nuclear Science and Engineering.

\section{Award}

- Certificate of Governance, American Nuclear Society Board of Directors (1985).

\section{B. L. McGill}

\section{Coordinator}

- MORSE-CGA Workshop, Holiday Inn, Oak Ridge, TN, June 17-21, 1985. 


\section{T. J. Mitchell}

Mesiber

- Five-Year Plan Committee for DOE Applied Mathematical Science Program.

\section{Invited papers}

- "Bicriteria Design Optimization," NSF Workshop on Efricient Data Collection, Berkeley, CA, July 18, 1984.

- "Designing Experiments on Computational Models," NSF Workshop on Efficient Data Collection, Berkeley, CA, July 19, 1984.

- "Multiobjective Optimization Algorithms in the Design of Experiments," SIAMASA-IASC Conference on Frontiers in Coraputational Statistics, Boston, MA, October 22, 1924.

- "Computer Construction of Supersaturated Screening Designs," NSF Workshop on Efficient Data Colkction, Berikeley, CA, February 11, 1985.

- "Computer Constuuction of Supersaturated Screening Designs," Conference on Statistical Theory and Practice, Madisor, WI, May 30, 1985.

D. L. Moses

Member

- Tower Shielding Reactor Subcommittee, ORNL Reactor Operations Review Committec.

\section{G. Ostrouchor}

Member

- Membership Committee, East Tennessee Chapter of the American Statistical Associatinn.

\section{Inrited paper}

- Symbolic Givens Reduction in Large Sparse Least Squares," SIAM Summer Meeting, Seattle, July 16-20, 1984.

Ph.D. thesis

- "Large Sparse Least Squares Computations," lowa State University, December 1984.

\section{Referee}

- The American Statistician

\section{R. W. Peelle}

\section{Member}

- Cross Section Evaluation Working Group (CSEWG) Executive Committee.

-. General Program Advisory Committec for 1985 International Conference on Nuclear Data for Basic and Applied Science.

- Technology Evaluation Committee, ORNL.

\section{Chairman}

- CSEWG Covariance Data Subcommittee.

\section{Invited talk}

- "Improving Neutron Reaction Data Through Linac-Based Flight-Time Spectrometry," Isotopes and Radiation Division, American Nuclear Society. Boston, MA, June 9-14, 1985. 


\section{Referee}

- The Physical Review

- Nuclear Science and Engineering

F. G. Perey

Mealer

- Nuclear Energy Agency Nuclear Data Committce (NEANDC).

\section{Chiman}

- NEANDC Task Force to resolve discrepancies in the Fe $1.15 \mathrm{keV}$ resonance parameters.

Invited paper

- Status of the Parameters of the 1.15-keV Resonance of ${ }^{j 6} \mathrm{Fe}$," International Conference on Nuclear Data for Basic and Applied Science, Santa Fe, New Mexico, May 13-17, 1985.

\section{Referee}

- Nuclear Science and Engineering

- Physical Review

- Nuclear Physics

\section{P. B. Perez}

\section{Member}

- Editorial Advisory Committee, Nuclear Science and Engineering journal.

\section{Professor}

- Nuclear Engineering Department, University of Tennessee, Knoxville.

\section{Referee}

- Nuclear Science and Engineering

- National Science Foundation

- The Physical Review

\section{A. Protopopescu}

\section{Invited lectures}

- The Abstract Time-Dependent Transport Problem," International Workshop on Operator Theory and lts Applications, Vrije Universiteit, Amsterdam, Húlland, June 3-7, 1985.

- "A Survey of Recent Results and Open Problems in Time-Dependent Transport," 9th International Conference on Transport Theory, Montecatini, Italy, June 10-14, 1985.

\section{Referee}

-.. Journal of Stotistical Physics

... SIAM Journal of Mathematical Analysis

-. Nuclear Science and Engineering

Transport Theory and Statistical Physics 
Reviewer

- Mathematical Reviews

\section{W. A. Rhoades}

Member

- Technical Program Committee, International Meeting on Advances in Nuclear Engineering Computational Methods, Knoxville, TN, April 9-11, 1985.

\section{Chairan}

- Exhibits Committee, International Meeting on Advances in Nuclear Engineering Computational Methods, Knoxville, TN, April 9-II, 1985.

Invited paper

- "Application of the Three Dimensional Oak Ridge Transport Code," ANS Topical Meeting on Reactor Physics and Shielding, Chicago, IL, September 17-19, 1984.

Arrard

- Best Paper Award, American Nuclear Society National Meeting, New Orleans, LA, June 1984.

R. W. Roussin

Member

- Panel on Reference Nuclear Data, representative for American Nuclear Society, Radiation Protection and Shielding Division.

- ANS 6.2.2: Shielding Benchmark Subcommittee.

- ANS 6.1.2: Shielding Cross Sections Committee.

\section{Chairman}

- Shielding Data Testing and Applications Subcommittec, Cross Section E raluation Working Group (CSEWG).

Vice Chairmxn/Chairman Elect

- Radiation Protection and Shielding Division, American Nuclear Srciety.

Invited papers

- "Status of Data Processing Codes and Data Libraries," Sixth Topical Meeting on the Technology of Fusion Energy, San Francisco, CA, March 1985

- "VITAMIN-E 174n, 38g Cross Section Library for Fusion Neutronics Calculations" (with J. E. White and J. L. Bartley), American Nuclear Society Meeting, New Orleans, LA, June 1984.

Referee

-.- Nuclear Science and Engineering

- Nuclear 7 echnology/Fusion

\section{R. T. Santoro}

Member

- Honors and Awards Committee, Radiation and Protection Division, American Nuclear Society.

- Honors and Awards Committee, Fusion Energy Division, American Nuclear Society.

- ORNL Traffic Committee. 
Fellow

- American Nuclear Society, June 1985.

\section{Referee}

- Nuclear Science and Engineering

- Nuclear Technology

- Nuclear Technology/Fusion

\section{L. Selby}

Member

- Technical Program Committee, International Meeting on Advances in Nuclear Engineering Computational Methods, Knoxville, TN, April 9-11, 1985.

\section{Cuainane}

- Registration Committee, International Topical Meeting on Fast Reactor Safety, Knoxville, TN, April 21-25, 1985.

- Arrangements Committec, International Meeting on Advances in Nuckear Engineering Computational Methods, Knoxville, TN, April 9-11, 1985.

- Oak Ridge/Knoxville Section of American Nuclear Society, 1985-86.

Invited paper

- Pressurized Thermal Shock Evaluation of Three Nuclear Power Plants," 12th Water Reactor Safety Research Information Meeting, Gaithersburg, MD, October 22-26, 1984.

\section{Award}

- Martin Marietta Technical Achievement Award, June 1985.

C. O. Slater

Member

- Program Committee of the ANS Radiation Protection and Shielding Division.

- ANS N.E.E.D. (Nuclear Engineering Education for the Disadvantaged) Committee.

- Program Committec for the 1984 Reactor Physics and Shielding Topical Meeting, Chicago, IL, September 17-19, 1984.

\section{A. D. Solomon}

\section{Invited papers}

- "Approximation Methods for Phase Change Problems," Meeting at Tel Aviv University, June 18, 1984.

- "Mathematical Methods in Latent Heat Storage," Meeting at Tel Aviv University, June 18, 1984.

- The Initial Speed of an Emerging Phase Change Front" (with V. Alexiades and D. G. Wilson), Meeting at Tel Aviv University, June 18, 1984.

-- "Heat Transfer Considerations in Passive Solar with Energy Storage" (with G. A. Geist et al.), Meeting at Tel Aviv University, June 18, 1984.

"A Model of a Direct Gain Passive Solar Structure with Latent Heat Storage" (with J. Tomlinson et al.), 1984 ASME/AICHE National Heat Transfer Conference, Niagara Falls, N. Y., August 1984. 
- The 1984 Model of Binary Alloy Solidification" (with D. G. Wilson et al.), Colloque International Problemes a Frontieres Libres, Maubuisson-Carcans, France, June 7-16, 1984.

- Modeling of Bulk Nucleation and Multiple Phase Front Formation in Pulsed Laser Melted Amorphous Silicon," Materials Research Society Symposium, Boston, MA, 1985.

\section{R. R. Spencer}

Member

- N.E.E.D. Committee of the American Nuclear Society.

- Program Committec for the 1984 Reactor Physics and Shielding Topical Meeting, American Nuclear Society.

\section{Referee}

- Nuclear Science and Engineering

- The Physical Review

Award

- Martin Marietta Publications Award, 1985.

\section{S. Thompson}

President

- Central Virginia Chapter, ACM, 1984-1985.

\section{Invited talk}

- "Event Detection in Continuous Simulation," International Conference on Power Plant Simulation, Cuernavaca, Morelos, Mexico, November 1984.

Referee

- Journal of the Society for Computer Simulation

\section{Reviewer}

- Computing

\section{R. N. Thurmer}

\section{Invited talk}

- "Imrroving RADEF; Annual Meeting of The American Civil Defense Association, Diytona Beach, FL, November 1984.

\section{R. D. Timmerman}

\section{Award}

- Best Paper Award, American Nuclear Society Student Conference, Gainesville, FL, April 6, 1985.

\section{K. Trubey}

\section{ANS repreneatative}

- ANSI Committee N17: Research Reactors, Reactor Physics, and Radiation Safety.

-. ANSI Committee N-13, Radiation Protection. 
Orivenan

- ANS-6: Radiation Protection and Shielding Subcommitter, Standards Committer, American Nuckar Society.

- ANS-6.4.3: Standards Working Group, Gamma-Ray Attenuation. Data.

- Telecommunications Subcommittec of Planning Committer, American Nuclear Society.

Mender

- Book Publishing Committce, American Nuckear Society.

- Executive Committee, Radiation Protection and Shielding Division, American Nuclear Society

$\because \because$ Irvited paper

- The Radiation Shielding Information Center: A Source of Computer Codes and Data for Dosimetry Applications" (with B. F. Maskewitz et al.), Health Physics Society Annual Meeting, New Orleans, LA, June 1984.

Conperter Secwits Ofincer

- Engineering Phy sics and Mathematics Division.

SI Standands Coortimator

-- Engineering Physics and Mathematies Division.

Referee

- Nuclear Science and Engineering

V. R. R. Uppoluri

Mencer

- American Statistical Association Committee on Nuclear Regulatory Research (1985).

Chimen

- Nominations Committee, Sigma Xi, Oak Ridge Chapter (1984-1985).

Tectuical Program Crainean

- 1984 Annual Meeting of the Society for Risk Analysis, Hyatt Regency Hotel, Knoxville, TN, Septeniber 30 - October 3, 1984.

Presillext

- East Tennewsee (hapter of the Society for Risk Analysis (1983-1984).

- Tennessee Mathematics and Computer Sciences Foundation (19\&5).

Invited papers

- "Fuzzy Hierarch cal Analysis" (with J. J. BuckJey), 1984 Annual Meeting, Society for Risk Analysis, Knoxville, TN, September 30 - October 3, 1984.

- "Estimation of the Minimur Latent Period for Radiogenic Oxtecuarcomas in Man," Symposium of the Radiobiulogy of Radium and Thorotrast, Neuherberg, FRG, October 29-31, 1984.

- A Simple Method for Assigning Uncertainties to Weights of Characteristics Based on Paired Comparisons" (with L. E. Lencester), Statistic Symposium on National Energy lssues, Seattle, WA, October 16-18, 1984. 


\section{Award}

- Society for Risk Analysis for service as the Chairperson. Technical Program, 1984 Annual Meeting.

\section{R. Voady}

\section{Menmer}

- Technical Program Committee, International Meeting on Advances in Nuclear Engineering Computational Methods, Knoxville, TN, April 9-1 I, 1985.

- Benchmark Committee, Mathematics and Computation Division, American Nuclear Society.

- ANS-10 Standards Committee, American Nuclear Societ".

- (Alternate) American National Standards Committec, X-3: Information Processing.

- (Alternate) Technical Committec, X3J3: Programming Language Fortran.

\section{Criman}

- Student Programs, International Meeting on Advances in Nuclear Engincering Computatiozal Methods, Knoxville, TN, April 9-11, 1985.

\section{Invited tall}

- "Computational Methods in Nuclear Reactor Analysis," Tennessee Industries Week. University of Tennessee, Knoxville, TN, September 10-14, 1984.

R. C. Ward

Member

- SIAM Council

- SIAM Program Committce

- ORNL Graduate Fellowship Selection Panel

\section{Co-Oriman}

-- SIAM Symposium on Frontiers in Computational Statistics.

\section{Program Cunirman}

- SIAM Activity Group on Linear Algebra.

Coortiantor

- ORNL Seminar Series on Parallel Computing.

Referee

- SIAM Joumal on Numerical Analysis

- IEEE Transactions on Auromatic Control

\section{R. Weisbin}

\section{Advary Elitor}

- Nuclear Science and Engineering.

\section{Member}

- Martin Marietta Corporate Headquarters Steering Committee on Artificial Intelligence. 
Caninan

- (Program) Second International Conference on Artificial Intelligense.

- DOE/CESAR Workshop: Planning and Sensing for Autonomous Navigation.

Director

-- Center for Engineering Systems Advanced Research (CESAR).

- Computer Science Department, University of Tennessec, Principles of Artificial intelligence."

\section{J. E. White}

\section{Me-wer}

- ANS-10 Subcommittee of the ANS Standards Committce.

\section{Invited paper}

- "VITAMIN-E 174n, 38g Cross-Section Library for Fusion Neutronics Calculations" (with R. W. Roussin and J. L. Bartley), ANS Meeting, New Orleans, LA, June 1984.

\section{G. Wilson}

Invited paper

- The 1984 Model of Binary Alloy Solidification" (with A. D. Solomon and V. Alexiades), Colloque International Problemes a Frontieres Libres, Maubuisson-Carcans, France, June 7-16, 1984.

\section{Invited talks}

- "Modeling of Solidification Processes," Seminar at the University of Tennessec, Knoxville, TN, May 23, 1984.

- "Computer Modeling of Solidification Processes," University of Miami, Coral Gables, FL, April 5, 1985.

- "Mathematical Modeling - Fun and Games with Applications," University of Tennessee, Chattanooga, TN, March 22, 1985.

-- Modeling Binary Alloy Solidification," SES Meeting, Blacksburg, VA, Octuber 17, 1984.

- "Modeling Binary Solidification," International Colloquiu n Free Boundary Problems, Maubuisson, Gironde, France, June 7-16, 1984.

- "Computer Modeling of Solidification Processes," Concast Service Union AG, Zurick, Switzerland, May 14, 1985.

\section{B. A. Worley}

\section{Member}

- Technical Program Committee, International Meeting on Advances in Nuclear Engineering Computational Methods, Knoxville, TN, April 9-11, 1985.

- Executive Board, Oak Ridge/Knoxville Section, American Nuclear Society.

\section{Chairman}

- Publicity Committee, International Meeting on Advances in Nucleaf Engineering Computational Methods, Knoxville, TN, April 9-11, 1985. 


\section{Secretary}

- Mathematics and Computation Division, American Nuclear Society.

\section{Referee}

- Nuclear Science and Engineering

- Nuclear Technology

\section{T. Wright}

\section{Member}

- American Statistical Association Advisory Committee to the U.S. Bureau of the Census.

- Review Panel of the U.S. Army Research Institute.

\section{Editorial Board}

- The American Journal of Mathematical and Management Sciences.

\section{Board of Directors and Treasurer}

- Tennessee Mathematics and Computer Sciences Foundation.

\section{Visiting Associate Professor}

- (Statistics) University of Tennessee, September 1984 - June 1985.

\section{Associate Professor}

- (Mathematies) Knoxville College, Knoxville, TN.

\section{ORNL Instructor}

- Statistical Methods for Quality Improvement, February 7 - April 10, 1984.

\section{Invited talk}

- "On Maximizing the Probability of Retention in PPS Sampling," Statistics Department, University of Tennessee, Knoxville, TN, March 7, 1984.

\section{Award}

- Distinguished Alumni of the Year Citation, National Association for Equal Opportunity in Higher Education, Washington, D.C., April 3, 1985.

\section{Referee}

- The American Journal of Mathematical and Management Sciences

- The American Statistician 


\section{ENGINEERING PHYSICS AND MATHEMATICS DIVISION SEMINARS AT ORNL}

Current seminar coordinators are D. C. Larson and M. C. G. Hall. The following seminars were held during January 1984 through June 1985.

J. Barhen, Engineering Physics and Mathematics Division, Supercomputing at ORNL: CESAR and the Hypercube Ensemble Project in Concurrent Processing"

D. E. Bartine, Engineering Physics and Mathematics Division, Developing Nuclear Power for Space Applications"

A. K. Bejczy, Jet Propulsion Laboratory, Pasadena, CA, Sensing and Control Intelligence in Robotics: Research and Perspectives"

W. Bickley, US Army Research Institute Field Unit, Fort Rucker, AL, Training and Cost Effectiveness of Initial Training Simulators"

R. Bottger, Physikalisch-Technische Bundesanstalt, Braunschweig, Federal Republic of Germany, "Neutron-Scattering Experiments at the PTB-Spectrometer"

G. P. Coddens, Universitaire Instelling Antwerpen, Antwerp, Belgium, Neutron, Alpha and Total Widths and Spin Assignments for Resonances in ${ }^{33} \mathrm{~S}+n$ from 10 to $400 \mathrm{keV}$

R. Y. Cusson, Department of Physics, Duke University, Durham, NC, "Low-Cost Computer Parallel Processing at ORNL"

G. F. Flanagan, Engineering Physics and Mathematics Division, Setting R\&D Priorities for DOE Liquid Metal Reactor Programs"

B. Gardner, IBM, Knoxville, TN, "IBM's Secrets Revealed at Last: Sierra Project Announcement"

R. R. Gawronski, University of West Florida, Learning Procedures for Dynamic Control of Manipulators and Robots with Application of Parallel Processing"

A. Geist, Engineering Physics and Mathematics Division, "Customizing UNIX"; and "An Introduction to Data Flow in Parallel Computers with a Simple Example"

A. George, Department of Computer Science, University of Waterloo, Waterloo, Ontario, Canada, "Solution of Large Sparse Systems of Equations"

C. M. Haaland, Engineering Physics and Mathematics Division, "Should We Protect Ourselves from Nuclear Weapon Effects?"

M. T. Heath, Engineering Physics and Mathematics Division, "An Overview of the Architecture of the Denelcor HEP Parallel Computer" 
F. Käppeler, Institute furr Kernphysik, Karlsruhe, Germany, Use of Barium Fluoride $\left(\mathrm{BaF}_{2}\right) \mathrm{Cry}$ stals in a Multicrystal Spectrometer for Measurements of Neutron Induced Reactions Important in the Study of Astrophysics and Nuclear Reactors"

G. L. Kaempf, Anacapa Sciences, Inc., "Current Research in U.S. Army Simulation"

H. V. Klapdor, Max Planck Institute, Heidelberg, Federal Republic of Germany, Neutrino Spectra in the Reactor Core and Reactor Decay Heat"

M. Leech, Computer Science Department, University of Tennessec, Knoxville, TN, Implementation of a Fuzzy Relational Data Base"

M. R. Leuze, Vanderbilt University, Nashville, TN, $\boldsymbol{K}_{b b}$ Matrix Orderings for Parallel Elimination"

R. M. A. Maayouf, Egyptian Atomic Energy Auth., Neutron Scattering in the Thermal Energy Region by Polycrystalline Materials and Single Crystals"

L. Martin and R. Spille, Instrumentation and Controls Divsion, "The Computer Architecture of the Advanced Servo-Manipulation System"

D. L. Matuszek, University of Tennessee, Knoxville, TN, Deriving Proof Rules for Control Structures"

T. E. McGuire, Health Systems International, Inc., New Haven, CT, Multi-tasking-real-timeFORTH at Kitt Peak National Observatory"

E. Ng, Department of Computer Science, University of Waterloo, Ontario, Canada, Static Implementation of Sparse Gaussian Elimination with Partial Pivoting"

D. P. O'Leary, Computer Science Department, University of Maryland, College Park, MD, Solution of Matrix Problems on Parallel Computers"

J. Ortega, University of Virginia, Charlottesville, VA, "A Survey of Vector and Paralle! Computers"; "An Introduction to Solving Linear Equations on Vector and Parallel Computers"; and "Multicolor Orderings for Iterative Methods"

M. Part, M/A-COM LINKABIT, Inc., Del Mar, CA, Multiprocessor-Based Power System Analysis"

F. G. Perey, Engineering Physics and Mathematics Division, "Introduction to the FPS-164/MAX; the FPS-164"

R. T. Schneider, University of Florida, Gainesville, FL, "Nuclear Pumped Lasers"

V. R. R. Uppuluri, Engineering Physics and Mathematics Division, The Distribution of the First $j$ Digits of Beta-Related Random Variables"

M. Wagner, Kraftwerk Union AG, Erlangen, Federal Republic of Germany, "Advances in Nodal Methods"

M. White, Manager of the DOE Technical Measurements Center, Albuquerque, NM, "Activities of the Technical Measurements Center of the DOE Division of Remedial Action Projects"

J. Worlton, Los Alamos National Laboratory, Los Alamos, NM (current assignment at the Office of ADP Management, DOE, Washington), Toward a Science of Parallel Computation" 


\section{PUBLICATIONS}

AGRAWAL, H. M.,** J. B. GARG,** AND J. A. HARVEY

Parity Dependence of the Level Densities of ${ }^{53} \mathrm{Cr}$ and ${ }^{55} \mathrm{Cr}$ at High Excitation," Phys. Rev. C 34Q, 1880 (1984). (1.28)

ALEXIADES, V., G. A. GEIST, AND A. D. SOLOMON

"Numerical Simulation of a HgCdTe Solidification Process," ORNL-6127 (August 1985). $(8.8)^{*}$

ALEXIADES, V., D. G. WILSON, AND A. D. SOLOMON

"Macroscopic Global Modeling of Binary Alloy Solidification Processes," Q. Appl. Math. 43(2), 143 (1985). (8.9)

ALSMILLER, F. S., T. A. GABRIEL, AND R. G. ALSMILLFR, JR.

"Hadron-Lepton Cascade Calculations (1-20 GeV) for a Pb-Al-Lucite Calorimeter," ORNL/TM-9153 (August 1984); Nucl. Instrum. Methods in Phys. Research A239, 175 (1985). (3.10)

ALSMILLER, R. G., JR., F. S. ALSMILLER, AND T. A. LEWIS**

"Calculations Pertaining to the Design of a Prebuncher for a 150-MeV Ebectron Linear Accelerator III. Comparisons with Experimental Data," ORNL/TM-9375 (November 1984); Part. Acceler. 17, 215, (1985). (1.59)

ALSMILlER, R. G., JR., J. BARHEN, J. E. HORWEDEL,** J. L. LUCIUS,** AND J. D. DRISCHLER

The Application of Adjoint Sensitivity Theory to a Liquid Fuels Supply Model," Energy Y(3), 239 (1984). (1983)

ALSMILLER, R. G., JR., J. E. HORWEDEL,** R. A. MARSHALLA,** D. M. NESBITT,** AND S. M. HAAS**

"A Model of the World Oil Market With an OPEC Cartel," ORNL/TM-9204 (August 1984); Energy 10, 1089 (1985). (3.15)

ALSMILLER, R. G., JR., R. T. SANTORO, J. F. MANNESCHMIDT, * AND J. M. BARNES**

"Calculations to Evaluate the Use of the Attenuation of D-D Neutrons in Liquid Oxygen as a Means of Measuring Plasma Ion Temperature," ORNL/TM-9230 (July 1984); Fusion Tech. 7, 197 (1985). (3.2)

\footnotetext{
-In most cases, the number shown in : entheses following the publica.son correaponds to the number of an abstract included in this report. In some casea, the number is a year, which means the abatract was published in an earlier progress report published that year. When no number is given, an abstract for the publication was not available.

- Not a member of Engineering Physics and Mathematics Division.
} 
AUCHAMPAUGH, G. F.,* M. S. MOORE ** J. D. MOSES,** R. O. NELSON,** R. C. EXTERMANN,* C. E. OLSEN,** N. W. HILL,* AND J. A. HARVEY

"Nature of the Coupling in Subthreshold Fission of ${ }^{238}{ }^{N p}$, Phys. Rev. C 29(1), 174 (i 984 ). (1.7)

BAYNE, C. K.,** J. J. BEAUCHAMP, AND V. E. KANE**

"Misclassification Probabilities for Second-Order Discriminant Functions Used to Classify Bivariate Normal Populations," Communications in Statistics B13, 669 (1984). (8.65)

BEALS, R."* AND V. PROTOPOPESCU

"Half Range Completeness and Orthogonality for the Fotker-Planck Equation with General Boundary Conditions II," Transp. Theory Stat. Phys. 13(1 \& 2), 43 (1984).

BEARE, A. N.,** M. D. DONOVAN,** D. L. LASSITER,** AND L. H. GRAY

The Effects of Supervisor Experience and Assistance of a Shift Technical Advisor (STA) on Crew Performance in Control Room Simulators," NUREG/CR-4280, ORNL/TM-9660 (September 1985). (5.6)

BEARE, A. N.,** R. E. DORRIS,** AND L. H. GRAY

Simulator Experiments: Effects of Experience of Senior Reactor Operators and of Presence of a Shift Technical Advisor on Performance in a Boiling Water Reactor Control Room," ORNL/TM-9327 (October 1984). (5.7)

BEAUCHAMP, J. J., AND V. E. KANE**

"Application of the Power-Shift Transformation," J. Statist. Comput. Simul. B19, 35 (1984). (8.66)

BEAUCHAMP, J. J., AND K. D. KUMAR**

"Subsampling Program for the Estimation of Fish Impingement," Environ Manage. 8, 539 (1984). (8.67)

BEER, H.,** R. L. MACKLIN

-198.199.200.201.202.204 $\mathrm{Hg}(n, \gamma)$ Cross Sections and the Termination of s-Process Nucleosynthesis," Phys. Rev C 32(3), 738 (1985). (1.22)

BEER, H.,* G. WALTER,"* R. L. MACKLIN, AND P. J. PATCHETT**

"Ne':ition Capture Crosi Sections and Solar Abundances of ${ }^{160,161} \mathrm{Dy},{ }^{170,171} \mathrm{Yb},{ }^{175.176} \mathrm{Lu}$, and ${ }^{176.177} \mathrm{Hf}$ for the s-Process Analysis of the Radionuclide ${ }^{176} \mathrm{Lu}$," Phys. Rev. C 30(2), 464 (1984). (1.20)

BERRY, M. W.,** M. T. HEATH, R. J. PLEMMiONS,** AND R. C. WARD

"Orthogonal Schemes for Structural Optimization," U.S. Army Research Office Report 84-1. Trans. First Army Conference on Applied Mathematics and Computing, p. 477 (1984). 8.40

BHUIYAN, S. I.,"* R. W. ROUSSIN, J. L. LUCIUS,** J. H. MARABLE, AND D. E. BARTINE

"Alternate Methods of Utilizing Cross-Section Sensitivity Coefficients in Radiation Shielding Problems," Nucl. Sci. Eng. 87, 96 (1984). (1983)

BOWMAN, K. O.

"Computation of the Polygamma Functions," Communications in Statistics B13(3), 409 (1984). (8.69)

"Extended Moment Series and the Parameters of the Negative Binomial Distribution," Biometrics 40, 249 (1984). (8.68) 
The Laboratory for Gastrointerology at Gunma University," Scientific Bulletin 10, 111 (1985).

Progress Report on Japanese Computer Projects," Scientific Bulletin 10, 91 (1985). (8.41)

BOWMAN, K. O., H. K. LAM,** AND L. R. SHENTON**

"Bounds for Certain Integrals," J. Cump. Appl. Math. 10, 245 (1984). (8.72)

"Bounds for Integrals Using Segments," J. Comp. Appl. Math. 11, 57 (1984). (8.73)

"Bounds for the Expectation of the kth Root of a Random Variable," J. Comp. Appl. Math 10, 229 (1984). (8.71)

BOWMAN, K. O., L. R. SHENTON,** AND S. R. BERNARD**

Study of Age Dependent Half-Life of Iodine in Man: A Reinforcement-Depletion Urn Model," Bull. Math. Biol. 47, 205 (1985). (8.77)

BOWMAN, K. O., L. R. SHENTON,** AND G. SZEKERES**

"A Poisson Sum Up to the Mean and a Ramanujan Problem," J. Stat. Comp. Simul. 20, 167 (1984). (8.79)

BRAU, J.,** T. A. GABRIEL, B. L. BISHOP,** AND J. HARGIS**

"Monte Carlo Studies of Uranium Calorimetry," ORNL/TM-9486 (January 1985); Nucl. Instru. Methods A238, 489 (1985). (3.11)

CACUCI, D. G., AND M. C. G. HALL

"Efficient Estimation of Feedback Effects with Application to Climatic Models," J. Atmos. Sci. 41(13), 2063 (1984). (4.38)

CACUCI, D. G., E. WACHOLDER, ** S. KAIZERMAN,"* AND N. TOMERIAN**

"Deterministic Sensitivity Analysis of Two-Phase Flow Systems: Forward and Adjoint Methods," EPRI NP-3635 (July 1984). (2.26)

CARLTON, R. F.,* W. M. GOOD,** J. A. HARVEY, R. L. MACKLIN, AND P. CASTEL **

Determination of Unbound States in ${ }^{35} S$ from Neutron Total and Capture Cross-Section Measurements on ${ }^{34}$ S," Phys. Rev. C 29'6), 1980 (1984). (1.9)

CARLTON, R. F.,* J. A. HARVEY, AND C. H. JOHNSON**

"s- and p-Wave Neutrons on ${ }^{30} \mathrm{Si}$ and ${ }^{\text {HA }} \mathrm{S}$ : Spherical Optical Model Analysis," Phys Rev. C $29,1988\left(19^{n}, 4\right)$. (1.24)

CARTER, B. J.,"* AND B. F. MASKEWITZ

"Abstracts of Digital Computer Code Packages Assembled by the Radiation Shielding Information Center," ORNL/RSIC-1 3, Vols. I-III/R (April 1985). (7.3)

CHAPMAN, G. T.,** R. T. SANTORO, R. G. ALSMILlER, JR., J. M. BARNES,** J. S. TANG,** AND P. D. SORAN**

"Fusion Reactor Shielding Benchmark 11. Duct Streaming Experiments and Analyses," Shielding Benchmark S (SBS) in Cross Section Evaluation Working Group Benchmark Specifications, BNL-19302 (ENDF-202), Vol. II, Deiember 1983, P. F. Rose and R. W. Roussin, Eds. (3.7)

CHRISTIE, N. T.,"* D. G. GOSSLEE, L. C. BATE,"* AND K. B. JACOBSON**

"Quantitative Aspects of Metal Ion Content and Toxicity in Drosophila," Toxicology 26, 295 (1983). (8.81) 
COLE, R. G.** T. KEYES, ** AND V. PROTOPOPESCU

Stationary Transport with Partially Reflecting Boundary Conditions,"J. Chem Phys. 81(0, 2771 (1984). (2C)

CRAMER, S. N.

"Applications Guide to the MORSE Monte Carlo Code," ORNL/TM-9355 (August 1985). (23)

"Applications Guide to the RSIC-Distributed Version of the KENO-V Code," ORNL/TM-9642 (September 1985). (29)

"Applications Guide to the RSIC-Distributed Version of the MCNP Code (Coupled Monte Carlo Neutron-Photon Code)," ORNL/TM-9641 (September 1985). (25)

DICKENS, J. K., N. W. HILL,** F. S. HOU,** J. W. MCCONNELL,** R. R SPENCER, AND F. Y. TSANG**

"An NE-213-Scintillator-Based Neutron Detection System for Diagnostic Measurements of Energy Spectra for Neutrons Having Energies $\geqslant 0.8 \mathrm{MeV}$ Created During Plasma Operations at the Princeton Tokamak Fusion Test Reactor," ORNL/TM-9561 (August 1985). (1.57)

DICKENS, J. K., AND D. C. LARSON

Fast Neutron Inelastic Scattering from "Cr," Bull. Am. Phys. Sac. 29, 1028 (1984). (1.2)

DIFILIPPO, F. C.

Stochastic Procesces in a Subcritical Nuclear Reactor in the Presence of a Fission Source," Nucl. Sci. Eng. 90, 13 (1985). (2.21)

'Comments on 'Remarks on the Inadequacy of One-Group Heterogeneous Theories for the Interpretation of In-core Neutron Noise in BWRs," Ann Nucl. Energy 11, 89 (1984). (1983)

DRESNER, L.,* P. FINN,"* J. BAUR,"* J. ROME,* C. SEGE, ** AND L. S. ABBOTT

Tnformal Handbook for Long-Term Exchangees in U.S.-Japan Cooperation Program in Fusion Research," Booklet, December 1983.

EMMETT, M. B.**

MORSE-CGA: A Monte Carlo Radiation Transport Code with Array Geometry Capability," ORNL-6174 (April 1985). (2.2)

The MORSE Monte Carlo Radiation Transport Code System," ORNL-4972/R2 (July 1984). (2.1)

FEDERMAN, P. J.,** W. D. BARTTER,"* AND A. I. SIEGEL**

"Job Analysis of the Electrician Position for the Nuclear Power Plant Maintenance Personnel Reliability Model," NUREG/CR-3275, ORNL/TM-8755, (February 1984). (1963)

FOGELBERG, B.,"* J. A. HARVEY, M. MIZUMOTO,"* AND S. RAMAN"*

Test of Fermi Gas Model Predictions of Level Density in ${ }^{137}$ Xe," Phys. Rev. C 31(6), 2041 (1985). (1.34)

FROME, E. L.

"Response to Nelder's Reaction on Poiseon Rate Analysis," Biometrics Journal 40, 1160 (1984). (8.83) 
FROME, E L, AND H. CHECKOWAY**

The Use of Poisson Regression Models in Estimating Incidence Rates and Ratios," Amer. J. Epidemiology 121(2), 309 (1985). (B84)

FU, C. Y.

Tmplementation of an Advanced Pairing Correction. for Particle-Hole State Densities in Precompound Nuctear Reaction Theory," Nucl. Sci. Eng. 86, 344 (1984). (1983)

FUNDERLIC, R. E, AND G. A. GEIST

Torus Data Flow for Parallel Computation of Missized Matrix Problems," ORNL-6125 (February 1985). (8.43)

FUNDERLIC, R. E, AND C. D. MEYER, JR.* *

Sensitivity of the Stationary Distribution " ytor for an Ergodic Markov Chain," ORNL6098 (January 1985). (8.44)

FUNDERLIC, R. E, AND R. J. PLEMMONS**

"A Combined Direct-Iterative Method for Certaiz M-Matrix Linear Systems," SIAM J. Alg. Disc. Meth S(1), 33 (1984); ORNL/CSD-112 (1983). (8.45)

GABRIEL, T. A., R. A. LILLIE, AND R. L. CHILDS**

The Use of Gd-Loaded Scintillation Detector Systems for Inverse Beta Decay Reactions," Nucl. Instrum. Letrs. 221, 474 (1984). (1983)

GEIST, G. A., AND M. T. HEATH

"Parallel Cholesky Factorization on a Hypercube Multiprocessor," ORNL-6190 (August 1985). (8.49)

GENEROSO, W. M.,** K. T. CAIN,** N. L. A. CACHEIRO,** C. v. CORNETT,"* AND D. G. GOSSLEE

Response of Mouse Spermatogonial Stem Cells to X-Ray Induction of Heritable Reciprocal Translocations," Mutation Research 126, 177 (1984). (8.87)

GEORGE, A.,** M. T. HEATH, AND J. LIU**

"Parallel Cholesky Factorization on a Multiprocessor," ORNL-61 24 (March 1985). (850)

GEORGE, A.,** M. T. HEATH, AND E. NG

Solution of Sparse Underdetermined Systems of Linear Equations," SIAM J. Sci. Stat. Comput. S(4), 988 (1984); ORNL/CSD-121 (1983). (8.51)

GEORGE, A.,"* AND J. LIU**

"Householder Reflections Versus Givens Rotations ir. Sparse Orthogonal Decomposition," Technical Report, Computer Science Department, Univerzity of Waterloo (1984). (8.52)

GEORGE, A.,** AND E. NG

"An Implementation of Gaussian Elimination with Partial Pivoting for Sparse Systems," SIAM J. Sci. Stat. Comput. G(2) 390 (1985). (8.55)

"Orthogonal Reduction of Sparse Matrices to Upper Triangular Form Using Householder Transformations," Technical Report CS-84-05, Computer Science Department, University of Waterloo (1984). (8.53)

Symbolic Factorization for Sparse Gaussian Elimination with Partial Pivotiog." Technical Report CS-84-43, Computer Science Department, University of Waterloo (1984). (8.54) 
GRAY, L. J., C. A. SERBIN, AND W. C. DIETRICH**

Simulation of the Electrochemical Machining Process," ORNL-6054 (November 1984). (8.13)

GWIN, R., R. R. SPENCER, AND R. W. INGLE**

"Measurements of the Energy Dependence or Prompt Neutron Emission from ${ }^{233} \mathrm{U}$, ${ }^{235} \mathrm{U}$, ${ }^{235} \mathrm{Pu}$, and ${ }^{211} \mathrm{Pu}$ for $E_{n}=0.005$ to $10 \mathrm{eV}$ Relative to Emission from Spontaneous Fission of ${ }^{252} \mathrm{Cr}, "$ Nucl. Sci. Eng. 87, 381 (1984). (1.3).

GWIN, R., R. R. SPENCER, R. W. INGLE** J. H. TODD,** AND S. W. SCOLES**

-Measurements of the Neutron Fission Cross Sections of ${ }^{235} \mathrm{U}\left(E_{n} \rightarrow 0.01 \mathrm{eV}\right.$ to $\left.30 \mathrm{keV}\right)$ and ${ }^{239} \mathrm{Pu}\left(E_{n}=0.01\right.$ to $\left.60 \mathrm{eV}\right)$," Nucl. Sci. Eng. 88, 37 (1984). (1.5)

HAALAND, C. M.

"Forecasting Radiation Exposure from Fallout Caused by Multiple, Nonsimultaneous, Upwind Ground Bursts," Health Phys. 46(2), 347 (1984). (2.51)

"Reply to Victor N. Evdokimoff," Health Phys. 48(4), 511 (1985). (2.50)

HALL, M. C. G., AND D. G. CACUCl

"Systematic Analysis of Climatic Model Sensitivity to Parameters and Processes," in Climate Processes and Climate Sensitivity, Maurice Ewing Vol. 5, 171 (1984). (1983)

HARVEY, J. A . C. H. JOHNSON,** R. F. CARLTON,** AND B. CASTEL**

"Single-Particle $2 d_{5 / 2}$ Strength in the ${ }^{18} \mathrm{Ca}+n$ Reaction," Phys. Rev. $C$ Rapid Communications 32(3), 1114 (1985). (1.27)

HEATH, M. T.

"Numerical Methods for Large Sparse Linear Least Squares Problems," SIAM J. Sci. Stat. Comput. 5(3), 497 (1984). (8.57)

Parallel Cholesky Factorization in Message-Passing Multiprocessor Environmer.:" ORNL-6150 (May 1985). (8.56)

HEATH, M. T., A. J. LAUB,** C. C. PAIGE,** AND R. C. WARD

"Computing the Singular Value Decomposition of a Product of Two Matrices," ORNL6118 (January 1985). (8.60)

HEATH, M. T., R. C. WARD, AND R. J. PLEMMONS**

"Sparse Orthogonal Schemes for Structural Optimization Using the Force Method," SIAM J. Sci. Stat. Comput. 5(3), 514 (1984); ORNL/CSD-119 (May 1983). (8.62)

HERMANN, O. W.,** AND T. J. BURNS

"Impact of Containment Building Leakage on LWR Accident Risk," NUREG/CR-3539, ORNL/TM-8964 (April 1984). (1983)

HETRICK, D. M.,** C. Y. FU, AND D. C. LARSON

Calculated Neutron-Induced Cross Sections for ${ }^{63.65} \mathrm{Cu}$ from I to $20 \mathrm{MeV}$ and Comparisons with Experiments," ORNL/TM-9083 (ENDF-337) (August 1984). (1.37)

HOLLAND, J. M.," AND E. L. FROME

"Statistical Evaluations in the Carcinogenesis Bioassay of Petroleum Hydrocarbons," Chapter 12 in Applied Toxicology of Petroleum Hydrocarbons, Vol. VI, pp. 15I, Princeton Scientific Publishers, Princeton, NJ, 1984. (8.90) 
HOREN, D. J.,** R. L. MACKLIN, J. A. HARVEY, AND N. W. HILL**

"Measurements of the Neutron Transmission and Capture Cross Sections in ${ }^{204} \mathrm{~Pb}$," Phys. Rev. C 25(6), 2126 (1984). (1.12)

HURST, G. S.,* M. G. PAYNE,** R. C. PHILlIPS,** J. W. T. DABBS, AND B. E. LEHMANN**

'Development of An Atom Buncher," J. Applied Phys. 55(5), 1278 (1984). (1983)

JOHNSON, J. O., AND T. J. BURNS

Neutronic Evaluation of Fissile Fuel Breeding Blankets for the Fission-Suppressed Tandem-Mirror Hybrid Reactor (TMHR)," ORNL/TM-8976 (June 1984). (1983)

JORGENSEN, C. C.

Tuclear Power Plant Personnel Qualifications and Training: TSORT - An Automated Technique to Assign Tasks to Training Strategies," NUREG/CR-3481, Vol. I, ORNL/TM-9308/VI (October 1984). (5.18)

Tuclear Power Plant Personnel Qualifications and Training: TAPS - The Task Analysis Profiling System," NUREG/CR-3481, Vol, 2, ORNL/TM-9308/V2 (June 1985). (5.20)

KAPLAN. T.,* AND L. J. GRAY

Self-Consistent Approximation for Muffin-Tiu Models of Random Substitutional Alloys with Environmental Disorder," Phys. Rev. B 29(6), 3684 (1984). (8.14)

KIRK, B. L.

"Beginner's Guide to Blocked Records on Magnetic Tape," ORNL/TM-9250 (September 1984). (7.7)

KIRK, B. L., AND J. T. WEST**

"Systems Guide to MCNP (Monte Carlo Neutron and Photon Transport Code)," ORNL/TM-9123 (June 1984). (7.8)

KLAUS, M.,* C. V. M. VAN DER MEE,** AND V. PROTOPOPFSCU

"Half-Range Solutions of Indefinite Sturm-Liouville Problems," C.R. Acad. Sc. Paris 300, I65, Series I (1985). (2.59)

KNEE, H. E., P. M. HAAS, S. L. PAINTER, AND M. D. ANDERSON

"CREDO: The Centralized Reliability Data Organization - A Data Base and Data Analysis Center for Advanced Reactors," Nuclear Safety 26(3), 300 (1985). (5.1)

KOZINSKY, E. J.,** L. H. GRAY, A. N. BEARE, ** D. B. BARKS, ** AND F. E. GOMER**

"Safety-Related Operator Actions: Methodology for Developing Criteria," NUREG/CR-3515, ORNL/TM-8942 (March 1984). (1983)

KUUSKRAA, V. A.,* F. MORRA,** R. G. ALSMILLER, JR., J. RARHEN, AND J. E. HOR WEDEL **

"Liquid Fuels Supply Model Data Base: Unconventional Recovery and Coal Liquefaction," ORNL/TM-8345 (March 1984). (1983)

LARSON, D. C.

"Reports on Nuclear Data Activities for the DOE Nuclear Data Committee," BNL-NCS34291, p. 138 (May 1984) and BNL-NCS-36272, p. 125 (May 1985). (1.48) 


\section{LARSON, D. C., AND R. C. HAIGHT**}

Ofince of Basic Energy Sciences Program to Meet High Priority Nuctear Data Needs of the Office of Fusion Energy: Summary of Results 1976-1983." ORNL/TM-9335 (September 1984). (1.4)

LARSON, D. C., N. M. LARSON,** AND J. A. HARVEY

ORELA Flight Path 1: Determinations of Its Effective Length vs Energy, Experimental Energies, and Energy Resolution Function and Their Uncertainties," ORNL/TM-8880 (June 1984). (1.60)

LARSON, D. C., N. M. LARSON,*" J. A. HARVEY, F. G. PEREY, D. E. PIERCE *" AND R. H. SEALS**

Taser Measurements of Distances from the ORELA Neutron Target to Experimeat Stations Along Flight Paths 1 and 6," ORNL/TM-9097 (March 1985). (1.61)

LARSON, N. M.**

'Updated Users' Guide for SAMMY: Multilevel R-Matrix Fits to Neutroa Data Using Bayes' Equations," ORNL/TM-9179 (August 1984), and ORNL/TM-9179/R] (April 1985). (1.53)

User's Guide for ALEX: Uncertainty Propagation from Raw Data to Final Results for ORELA Transmission Measurements," ORNL/TM-8676 (ENDF-332) (February 1984). (1.55)

LAUGHERY, K. R.**

-A Select Review of the Recent (1979-1983) Behavioral Research Literature on Training Simulators," NUREG/CR-4206, ORNL/TM-9445 (May 1985). (5.22)

LIU, J. W. H.**

"On General Row Merging Schemes for Sparse Givens Transformations," Technical Report CS-83-04, Computer Science Department, York University, November 1984. (8.63)

LOCK, S * W. DALBEY,** R. L. SCHMOYER, AND R. GRIESEMER** alation Toxicology of Diesel Fuel Obscurant in Sprague-Dawley Rats," ORNL/TM-9403, May 1984. (8.92)

MACKLIN, R. L.

"Neutron Capture Measurements on Fission Product Palladium-107," Nucl. Sci. Eng. 89, 79 (1985). (1.19)

Neutron Capture Measurements on Radioactive ${ }^{93} \mathrm{Zr}$," Astrophysics and Space Science 115, 71 (1985). (1.18)

Resonance Neutron Capture by ${ }^{39.41} \mathrm{~K},{ }^{-}$Nucl. Sci. Eng. 88, 129 (1984). (1.15)

Resonance Neutron Capture by ${ }^{35.37} \mathrm{Cl}, "$ Phys. Rev. C 29(0), 1996 (1984). (1.14)

Resonance Neutron Capture by Manganese Below 2.5 keV," Nucl. Sci. Eng. 89, 362 (1985). (1.16)

"Neutron Capture Cross Sections of Tantalum from 2.6 to $1900 \mathrm{keV,"} \mathrm{Nucl.} \mathrm{Sci.} \mathrm{Eng.} 86$. 362 (1984). (1983)

MACKLIN, R. L., AND S. F. MUGHABGHAB**

Teutron Capture by ${ }^{31}$ P," Phys. Rev. C 32, 379 (1985). (1.13) 
MAERKER, R. E

Gamma-Ray Characterization of the Two-Year Irradiation Experiment Performed at the Poolside Facility," NUREG/CR-4039, ORNL/TM-9440 (January 1985). (415)

MAERKER, R. E, B. L. BRCIADHEAD,** AND J. J. WAGSCHAL**

Theory of a New Unfolding Procedure in PWR Pressure Veased Dosimetry and Derelopment of an Associated Benchmart Database, Nucl. Sa. Eng. 91, 369 (1985). (4.17)

MAERKER, R. E, W. E FORD, II, ** C. C. WEBSTER, ** AND M. L. WILLIAMS**

The ELXSIR Cross-section Library for LWR Pressure Veasel Irradintion Stodies. Part of the LEPRICON Ccmputer Code System," EPRI NP-3654 (September 1984). (419)

MAERKER, R. E, M. L. WILLIAMS,** B. L. BROADHEAD,** J. J. WAGSCHAL,** AND C. Y. FU

Revision and Expansion of the Data Base in the LEPRICON Dosimetry Methodolozy," EPRI NP-3841 (January 1985). (4.18)

MAERKER, R. E, AND B. A. WORLEY

"Activity and Fluence Calculations for the Startup and Two-Year Irradiation Experiments Performed at the Poolside Facility," NUREG/CR-3886, ORNL/TM-9265 (October 1984). (4.12)

MAGUIRE, H. T., JR.,* C. R. S. STOPA,* R. C. BLOCK,** D. R. HARRIS,** R. E. SLOVACEK, $*$ J. W. T. DABBS, R. J. DOUGAN, ** R. W. HOFF,"* AND R. W. LOUGHEED**

"Neutron-Induced Fission Cross-Section Measurements of ${ }^{244} \mathrm{Cm},{ }^{246} \mathrm{Cm}$, and ${ }^{246} \mathrm{Cm}$," Nucl. Sci. Eng. 89, 293 (1985). (1.8)

MARCH-LEUBA, J.,* D. G. CACUCI, AND R. B. PEREZ

Universality and Aperiodic Behavior of Vuclear Reactors," Nucl. Sci. Eng. 86, 401 (1984). (1983)

MASKEWITZ, B. F., J. A. WATTS, $*$ C. R. WEISBIN, L. J. ALLISON, $* *$ B. F. JACOBS,** AND H. A. PFUDERER**

"Carbon Dioxide Information Center (CDIC): A Progress Report for the Period January 1982 - September 1983," ORNL/TM-9350 (October 1984). (7.12)

MERRIMAN, L. D.**

"Fission-Product Yields for Thermal-Neutron Fission of ${ }^{243} \mathrm{Cm}$ Determined from Measurements with a Hig 1-Resolution Low-Energy Germanium Gamma-Ray Detector," ORNL/TM-9049 (April 1984). (1.36)

McMAHAN, C. A.,"* AND M. D. MORRIS

"Application of Maxisnum Likelihood Paired Comparicon Ranking to Estimation of a Linear Don:inance Hierarchy in Animal Societies," Anim. Behav. 32, 374 (1984). (8.S4)

MINCER, A. I.,** T. A. GABRIEL, B. L. BISHOP,** H. T. FREUDENREICH, ** J. A. GOODMAN,"* S. C. TONIVAR,"* G. B. YODH*" D. BERLEY,* AND R. W. ELLSWORTH*0

"A Monte Carlo Simulation of the Response of a Hadronic Calorimeter to Protons of Momentum 3.5 to $200 \mathrm{GeV} / c$;" ORNL/TM-9270 (January 1985); Nucl. Instrum. Methods A238, 482 (1985). (3.12)

MONTAGUE, D. F,"* J. B. FUSSELL,"* P. A. KROIS, T. C. MORELOCK,"* H. E. KNEE, J. J. MANNING, P. M. HAAS, AND K. W. WEST**

"A Review of the Treat Upgrade Reactor Scram System Reliability Analysis," ORNL/TM-9367 (October 1984). (5.4) 
MORRIS, M. D.

"Minimum Number of Runs for Two-Level Factorial Search Designs," J. Statistical Planning and Inference 10, 115 (1984). 1290)

MORRIS, M. D., AND S. F. EBEY**

"An Interesting Property of the Sample Mean Under a First-Order Autoregressive Model," The American Statistician 30(2), 127 (1984). (8.97)

MUCKENTHALER, $\boldsymbol{r} . \mathbf{J}$.

Integral Measurements of Neutron and Gamma-Ray Leakage Fluxes from the Little Boy Replica," ORNL/TM-900S (March 1984). (1983)

Phase I Measurements for the HTGR Bottom Reflector and Core Support Block Neutron-Streaming Experiment," ORNL/TM-8977 (July 1984). (1983)

OBLOW, E. M.

"GRESS - Gradient-Enhanced Software System, Version D User's Guide," ORNL/TM-9658 (July 1985). (4.34)

OBLOW, E. M., AND F. G. PEREY

"Screening Sensitivity Theory," Nucl. Sci. Eng. 87, 444 (1984). (1983)

OLSEN, D. $K$.

"Report to the ${ }^{238} \mathrm{U}$ Discrepancy Task =nrce on SIOB Fits to the ORNL, CBNM, and JAERI Transmission Data," ORNL/TM 2023 (ENDF-338) (May 1984). (1.44)

OLSEN, D. K., J. A. MARTIN,** AND D. J. H. :EN**

"A Preliminary Study of Possible ORELA ieplacement Options," ORNL/TM-86ó9 (June 1984). (1.58)

OSTROUCHOV, G.

Symbolic Givens Reduction in Large Sparse Least Squares Problems," ORNL-6102 (December 1984). (8.101'

PIN, F. G., J. P. WITHERSPOON,** D. W. LEE,** J. B. CANNON,** AND R. H. KETELLE**

"Radionuclide Migration Pathways Analysis for the Oak Ridge Central Waste Disposal Facility on the West Chestnut Ridge Site," ORNL/TM-9231 (October 1984). (4.30)

POENITZ, W. P.,"* AND G. De SAUSSURE

The Nuclear Data of the Major Actinide Fuel Materials," Progress in Nucl. Energy 13, 129 (1984). (1983)

PROTOPOPESCU, $v$.

"On the Spectral Decomposition of the Transport Operator with Anisotropic Scattering and Periodic Boundary Conditions," Transp. Theory Stat. Phys. 141), 103 (1985). (2.55)

PROTOPOPESCU, V., AND T. KEYES*

The Goldstein-McKean Model Revisited," Physica A. 132, 421 (1985). (2.57)

ROLISSIN, R. W.

"Structural, Heavy Coolant, and Material Cross-Section Data," Progress in Nucl. Energy 13, 235 (1984). (1983) 
SANTORO, R. T., J. M. BARNES, ** R. G. ALSMILLER, JR., AND J. D. DRISCHLER

Monte Carlo and Discrete Ordinates Calculations of 14-MeV Neutrons Streaming Through a Stainless-Steel Duct: Comparison with Experiment I," ORNL/TM-9541 (March 1985). (3.1)

SANTORO, R. T., J. M. BARNES, ** J. D. DRISCHLER, AND R. G. ALSMILLER, JR.

Multigroup Energy-Angle Distributions for Neutrons from the $T(d, n)^{4}$ He Reaction $\left(E_{d}=\right.$ $100-400 \mathrm{keV}$ )," ORNL/TM-9251 (July 1984). (3.3)

SANTORO, R. T., N. A. UCKAN,** J. M. BARNES, ** AND D. E. DRIEMEYER**

"An EBT Reactor Systems Analysis and Cost Code: Description and User's Guide (Version 1)," ORNL/TM-9026 (June 1984). (3.6)

SANTORO, R. T., N. A. UCKAN,** AND R. J. SCHMITT**

"Magnetics Calculations for an ELMO Bumpy Square," ORNL/TM-9542 (March 1985). (3.5)

SANTORO, R. T., J. E. WHITE, AND J. D. DRISCHLER

"ORACT: A 174-Neutron-Group Activation Cross-Section Library for Fusion and Fission Reactor Design Studies," ORNL/TM-9203 (June 1984). (3.4)

SAYAGE, M. G.*

-A One-Dimensional Modeling of Radial Heat Removal During Depressurized Heatup Transients in Modular Pebble-Bed and Prismatic High-Temperature Gas-Cooled Reactors," ORNL/TM-9215 (July 1984). (2.24)

SCHMOYER, R. L.

"Everyday Application of the Cell Means Model," The American Statistician 38(1), 49 (1984). (8.105)

"Sigmoidally Constrained Maximum Likelihood Estimation in Quantal Bioassay," J. Am. Srat. Assoc. 79, 448 (1984). (8.104)

SCOTT, D. S."* M. T. HEATH, AND R. C. WARD

Parallel Block Jacobi Eigenvalue A lgorithms Using Systolic Arrays," Technical Report CS-85-01, University of Texas, Computer Science Dept. (1985). (8.64)

SELBY, D. L., AND W. T. HENSLEY

"A Ranking Scheme for Making Decisions on the Relative Training Importance of Potential Nuclear Power Plant Malfunctions," NUREG/CR-3523, ORNL/TM-8950 (January 1984). (1983)

SIEGEL, A. I.," W. D. BARTTER," J. J. WOLF," AND H. E. KNEE

"Maintenance Personnel Performance Simulation (MAPPS) Model: Description of Model Content, Structure, and Sensitivity Testing," NUREG/CR-3626, Vol. 2 , ORNL/TM-9041/V2 (December 1984). (5.25)

SIEGEL, A. I.," W. D. BARTTER,"* J. J. WOLF,"* H. E. KNEE, AND P. M. HAAS

Maintenance Personnel Performance Simulation (MAPPS) Modei: Summary Description," NUREG/CR-3626. Vol. I, ORNL/TM-9041/VI (May 1984). (5.27)

SLATER, C. $O$.

"Analysis of Phase I of the HTGR Bottom Reflector and Core Support Block NeutronStreaming Experiment," ORNL/TM-9252 (July 1984). (2.17) 
SOBEL, M.,* S. R. BERNARD,** V. R. R. UPPULURI, AND C. W. NESTOR, JR.**

"Four-Urn Catenary Model for Excretion," Bull. Math Biol. 46, 219 (1984). (8.110)

SOLOMON, A. D.

The Mathematics of Artificial Intelligence," ORNL Review 17, 28 (1984). (8.16)

SOLOMON, A. D., V. ALEXIADES, AND D. G. WILSON

The Initial Velocity of the Emerging Free Bounoary in a Two-Phase Stef?n Problem with Imposed Flux," ORNL-6073 (November 1984). (8.23)

SOLOMON, A. D., V. ALEXIADES, AND D. G. WILSON

"Moving Boundary Problems in Phase Change Models: Current Research Questions," Signum Newsletter 20(2), 8 (1985). (8.25)

SOLOMON, A. D., V. ALEXIADES, D. G. WILSON, AND J. DRAKE

The Formulation of a Hyperbolic Stefan Problem," ORNL-6065 (October :1984); Quarterly of Applied Mathematics XLIII(3), 295 (1985). (8.20)

SOLOMON, K. A.,"* W. E. KASTENBERG,** AND P. F. NELSON**

'Dealing with Uncertainty Arising Out of Probabilistic Risk Assessment," ORNL/TM-9076 (March 1984). (1983)

SOLOMON, A. D., D. G. WILSON, AND V. ALEXIADES

The Quaisi-Stationary Approximation for the Stefan Problem with a Convective Boundary Condition," Intern. J. Math. and Math. Sciences 7(3), 549 (1984). (8.29)

STORER, J. B.,** AND T. J. MITCHELL

Limiting Values for the RBE of Fission Neutrons at Low Doses for Life Shortening in Mice," Radiation Research 97, 396 (1984). (8.111)

SUBBAIAH, K. V.,** A. NATARAJAN,** D. V. GOPINATH,** AND D. K. TRUBEY

Effect of Fluorescence, Bremsstrahlung, and Annihilation Radiation on the Spectra and Energy Deposition of Gamma Rays in Bulk Media," Nucl. Sci. Eng. 81, 172 (1982). (7.11)

TODD, J. H.,** L. W. WESTON, AND G. J. DIXON**

"A Parallel Plate ${ }^{10}$ B Neutron Detector," Nucl. Instrum. Methods 219, 575 (1984). (1983)

TRUBEY, D. K., B. F. MASKEWITZ, AND R. W. ROUSSIN

The Radiation Shielding Information Center: A Source of Computer Codes and Data for Dosimetry Applications," Health Phys. 47, 198 (1984). (7.1)

T:JRNBULL, B. W.,** AND T. J. MITCHELL

"Nonparametric Estimation of the Distribution of Time to Onset for Specific Diseases in Survival/Sacrifice Experiments," Biometrics 40, 41 (1984). (8.114)

UPPULURI, V. R. R., AND S. A. PATIL

The Distribution of the First $j$ Digits of Beta Related Random Variables," Commun Statist. -. Simula. Computa. 14(2), 467 (1985). (8.116)

VONDY, D. R.

"A Multigrid Iteration Solution Procedure for Solving Two-Dimensional Sets of Coupled Equations," ORNL/TM-9030 (July 1984). (2.36) 
"A Multigrid Iteration Solution Procedure for Solving Three-Dimensional Sets of Coupled Equations," ORNL/TM-9248 (August 1984). (2.37)

"Benchmark Problem Solution: Two-Dimensional PWR Burnup Problem with Fuel Management Treating Two Cycles," Argonne Code Center: Benchmark Problem Book, ANL-7416 (1985). (233)

THR-TH: A High-Temperature Gas-Cooled Nuclear Reactor Core Thermal Hydraulics Code," ORNL-5951 (July 1984). (234)

2240-MW(th) High-Temperature Reactor Core Power Density Study," ORNL/TM-9099 (September 1984). (235)

"Kegarding Overrelaxation for Accelerating an Iteration Process," ORNL/TM-8922 (June 1984). (238)

WACHOLDER, E, ** S. KAIZERMAN,** N. TOMERIAN,** AND D. G. CACUCI

"An Exact Sensitivity Analysis of a Simplified Transient Two-Phase Flow Problem," Nucl. Sci. Eng. 89, 1 (1985). (2.27)

WEISBIN, C. R., AND K. McKEEHAN**

"Center for Engineering Systems Advanced Research Program," Brochure, February 1985. (4.2)

WESTON, L. W., AND J. H. TODD**

Subthreshold Fission Cross Section of ${ }^{200} \mathrm{Pu}$ and the Fission Cross Sections of ${ }^{235} \mathrm{U}$ and ${ }^{239}$ Pu," Nucl. Sci. Eng. 88, 567 (1984). (1.6)

WHITE, J. R.**

The Depth-Charge Static and Time-Dependent Perturbation/Sensitivity System for Nuclear Reactor Core Analysis," ORNL/CSD-78/RI (April 1985). (239)

WILLIAMS, M. L., ** F. W. STALLMANN,** R. E. MAERKER, AND F. B. K. KAM**

Validation of Neutron Transport Calculations in Benchmark Facilities for Improved Damage Fluence Predictions," Nucl. Eng. Design 86, 87 (1985). (4.16)

WILSON, D. G.

Time Dependent Linea: Trar. sport III - Convergence of the Discrete Ordinates Method," Transp. Theory Stal. Phys. 12(4), 369 (1984). (8.32)

WILSON, D. G., A. D. SOLOMON, AND V. ALEXIADES

"A Model of Binary Alloy Solidification," Int. J. Num. Meth. in Engr. 20, 1067 (1984); ORNL/CSD-97 (August 1982). (8.38)

WISSHAK, K.,** F. KAPPELER,** R. L. MACKLIN, G. REFFO,** AND F. FABBRI**

"Neutron Capture in s-Wave Resonances of Nickel-64," Nucl. Sci. Eng. 87, 48 (1984). (1983)

WORLEY, B. A.

"Improvements in Calculating Neutron Transmission Probabilities in Unit-Cell InterfaceCurrent Codes," Nucl. Sci. Eng. 91, 293 (1985). (4.29)

WRIGHT, T. (Ed.)

Statistical Methods and the Improvement of Data Quality. Academic Press, New York, New York (1983). (8.117) 
WRIGHT, T., AND H. J. TSAO**

"On an Optimal Solution for Maximizing the Probability of Retention in PPS Sampling," Linear Algebra and Its Applications 67, 67 (1985). (8.119)

Some Useful Notes on Simple Random Sampling," J. of Quality Tech. i7(2), 67 (1985). (8.118) 


\section{PAPERS PRESENTED AT SCIENTIFIC MEETINGS AND SEMINARS}

EPRI/BNL Workshop on Methods of Assessing Health Impacts, Uptom, NY, December 7-8, 1981

RHYNE, W. R.,"* AND A. A. EL-BASSIONI,** "A Methodology for Comparing the Health Effects of Electricity Generation from Uranium and Coal Fuels." (6.2)"

Laboratory Seminar, Los Alamos National Laboratory, January 16, 1983; Sandia National Laboratory, January 17, 1983; Lawrence Livermore National Laboratory, January 27, 1983

MASKEWITZ, B. F., D. K. TRUBEY, AND R. W. ROUSSIN, The Radiation Shielding Information Center - A Technology Resource for the DOE Weadons Laboratories."

Japan Carbon Dioxide Research Committee Meeting, University of Toky,, Tokyo, Japan, May 12, 1983: Academy of Meteorological Science, Beijing. China, May 27, 1983

MASKEWITZ, B. F., " $\mathrm{CO}_{2}$-Climate Research in the United States and the Program of the CDIC."

Lectures to technical personnel from several nuclear installations at the Institute of Atomic Energy, Beijing (Tuoli). China, May 23-26. 1983; summary lecture. Beijing Institute of Nuclear Engineering, May 30, 1983; lecture at 728 Research and Design Institute, Shanghai, June I-3, 1983

MASKEWITZ, B. F., "State of the Art Methodology - Radiation Protection, Transport, and Shielding."

Little Boy Colloquium, Los Alamos National Laboratory. Los Alamos, NM. September 13-14, 1983

MUCKENTHALER, F. J., "Integral Neutron and Gamma-Ray Measurements of the Leakage Flux from a Mockup of Little Boy." (2.13)

DOE Conference on "CO 2 Science and Consensus," Coolfont, Berkeley Springs, West Virginia, Seprember 19-23, 1983

MASKEWITZ, B. F., J. A. WATTS,** AND C. R. WEISBIN, The Carbon Dioxide Information Center - Operational Overview, Resources, and Potential Support."

\footnotetext{
- In most cases, the number shown in parentheses following the publication corresponds to the number of an abstract included in this report. In some cases, the number is a year, which means the abatract was published in an earlier progress report published that year. When no number is given, an abstract for the publication was not available.

- Not a member of Engineering Physics and Mathematics Division.
} 
ASA/ISAC/SIAM Conference on Frontiers in Computational Statistics, Baston, MA, 1981

BOWMAN, K. O., Progress Report on Super Computers and Statistical Computing in Japan."

First Sympasium on Space Nuclear Power Systems, Albuquerque, NM. January 10-13, 1984

MASKEWITZ, B. F., "RSIC - A Technology Resource for Space Nuclear Power Systems."

Statistics Department, University of Tennessee, Knoxville, TN, March 7, 1984

WRIGHT, T., On Maximizing the Probability of Retention in PPS Sampling." (8.119)

Eastern North American Regional Spring Meeting of the Biometric Society, Orlando, FL, March $11-14,1984$

SCHMOYER, R. L., J. J. BEAUCHAMP, AND J. F. MCCARTHY,"* "A Nonparametric Analysis for Steep Dose-Response Bioassays " (8.107)

American Association of Physicists in Medicine First Midyear Topical Symposium on Multiple Regression Analysis: Applications in the Health Sciences, Mobile, AL. March I2-16, 1984

FROME, E. L., "Regression Methods for Binomial and Poisson Distributed Data." (8.82)

Ninth SAS Users Group International Conference, Cany. NC, March 18-21, 1984; Proc. Stat. Anal. Syst. Users Group Int. (1984)

FROME, E. L., AND R. McLAIN,** "A Macto for Fitting Nonlinear Models to Poisson Distributed Data," p. 883. (8.86)

Numerical Computation and Mathematical Software for Microcomputers Conference, Boulder. CO. March 19-21, 1984

GAFF'EY, P. W., FORTRAN Programming on an IBM PC." (8.47)

1984 Annual Meeting of the American Physical Society, Detroit, MI, March 27, 1984

HAALAND, C. M., Should We Protect Ourselves from Nuclear Weapons Effects?" (2.52)

Silver Anniversary Celebration of the Michigan Section, American Nuclear Society. Amn Arbor, MI, April 1I. 1984

MASKEWITZ, B. F., "Nuclear Power - Myth vs. Reality."

Biometry Branch Seminar, National Institute of Environmental Health Science Research, Triangle Park, NC, April 13,1981

MORRIS, M. D., AND S. F. EBEY, "On the Precision of the Average of Correlated Measurements." (8.98)

Modeling and Simulation Conference, Pittsburgh, PA, April 19-20, 1984

JANARDAN, K. G."* AND V. R. R. UPPULURI, "A Stochastic Model for Estimation of Environmental Density." (8.91) 
Federation of the American Society for Experimental Biology, Anaheim, CA, April 2!-26, 1984

M. SOBEL,** S. R. BERNARD,** AND V. R. R. UPPULURI, "On the Distribution of the Time Required to Remove White Balls from an Urn." (8.109)

1984 Conference on Intelligent Systems and Machines, Oakland University. Rochester, MI, April 24-25. 1984

WEISBIN, C. R., G. de SALSSURE, AND J. BARHEN, "Automated Planning for Intelligent Machines in Energy-Related Applications." (4.4)

ORAU Traveling Lecture, Williamsburg, VA, April 27, 1984

GRAY, L. J., "Self-Consistent Approximations for Random Alloys." (8.I1)

Colloquium, NASA, Marshall Space Flight Center, Huntsville, AL, May 1984

ALEXIADES, V., D. G. WILSON, AND A. D. SOLOMON, "A General Model of Binary Alloy Solidification." (8.6)

Conference on Inorganics in Drinking Water and Cardiovascular Disease. University of Massachusetts, Amherst, MA, May 1-3, 1984

ZEIGHAMI, E. A.,"* M. D. MORRIS, E. E. CALLE,*" F. S. McSWEENY,** AND B. A. SCHUKNECHT,"* "Drinking Water Inorganics and Cardiovascular Disease: A CaseControl Study Among Wisconsin Farmers." (8.120)

U.S. DOE/Japan PNC Bilateral Exchange Agreement for Shielding Data, Tokyo. Japan, May $11-13,1984$

BARTINE, D. E., "Highlights of Shielding Analysis and Integral Experiments Performed for the U.S. Fast Flux Test Facility."

National Energy Software Center Installation Representatives Meeting, Argonne National Laboratory. May 15-16. 1984

MASKEWITZ, B. F., "The RSIC's Specialized Collections."

Meeting at the University of Tennessee, Knoxville, TN, May 23, 1984

WILSON, D. G., "Computer Modeling of Solidification Processes." (8.36)

1984 Annual Summer Meeting of the American Nuclear Society, New Orleans, LA. June 3-8. 1984; Trans. Am. Nucl. Soc. 16 (1984)

BROADHEAD, B. L.,"* D. G. CACUCI, AND J. V. PACE, III,** "Adjoint Transport Calculations for Sensitivity Analysis of the Hiroshima Air-Over-Ground Environment," p. 638. (2.46)

BROADHEAD, B. L.,** AND R. E. MAERKER, "Sensitivities of the Flux Spectrum in the Cavity of a PWR to Variations in the Core Source Distribution," p. 659. (4.26)

CACUCI, D. G., E. WACHOLDER,** S. KAIZERMAN,** AND N. TOMERIAN,** "Adjoint Sensitivity Analysis of a Thermal-Hydraulic System Undergoing Phase Change Due to Boiling Transition," p. 870. (2.28) 
CHILDS, R. L.,** AND W. A. RHOADES, The Linear Nodal Method for Shielding Applications," p. 657. (2.9)

DIFILIPPO, F. C., "A Theory of the Fission-Source-Driven Neutron Noise Field," p. 738. (2.18)

ENGLE, W. W., JR., AND D. E. BARTINE, "Comparison of Candidate Shielding Materials for Space Power Reactor Applications," p. 335. (2.12)

GRAY, L. H., P. M. HAAS, J. J. MANNING, AND J. C. LOWRY,"* "Control Room Operator Performance Research," p. 309. (5.9)

HAAS, P. M., C. C. JORGENSEN, D. L. SELBY, AND J. C. LOWRY,** "Methods for Evaluation of Nuclear Power Plant Personnel Training and Entry Level Qualifications," p. 307. (5.17)

MAERKER, R. E., AND B. A. WORLEY, Neutron Calculations of the Two-Year Irradiation Experiment at the ORR-PSF and Comparison with Measurements," p. 634. (4.14)

MARCH-LEUBA, J.,* R. B. PEREZ, AND D. G. CACUCI, "Calculation of Limit Cycle Amplitudes in Commercial Boiling Water Reactors," p. 749. (2.31)

ROUSSIN, R. W., J. E. WHITE, AND J. L. BARTLEY, VITAMIN-E 174-Neutron, 38-Gamma Cross-Section Library for Fusion Neutronics Calculations," p. 272. (7.6)

SAVAGE, M. G."* AND B. A. WORLEY, "Modular Gas-Cooled Reactor Heat Transfer Mechanisms," p. 874. (2.23)

SIEGEL, A. I.,* W. D. BARTTER,** J. J. WOLF,** H. E. KNEE, AND P. M. HAAS, The Maintenance Personnel Performance Simulation (MAPPS) Model," p. 298. (5.26)

SIU, N.,"* AND G. APOSTOLAKIS,"* "Modeling the Detection Rates of Fires in Nuclear Power Plants," p. 537. (6.3)

SLATER, C. O., "Analysis of Phase I of the ORNL HTGR Bottom Reflector and Support Block Neutron Streaming Experiment," p. 637. (2.17)

WOOD, R. T.,* J. MARCH-LEUBA,** R. B. PEREZ, F. J. SWEENEY,** "A Stochastic Model to Monitor Mechanical Jibrations in Pressurized Water Reactors," p. 735. (2.32)

Health Physics Society .Annual Meeting. New Orleans, LA, June 3-8, 1984

HAALAND, C M., "A Simple Graphical Method for Forecasting Radiation Exposure from Multi-Ag:d Fallout from Nuclear Weapons." (2.53)

International Colloquium on Free Boundary Problems, Maubuisson, Gironde, France, June 7-15, 1984

WILSON, D. G., V. ALEXIADES, AND A. D. SOLOMON, The 1984 Model of Binary Alloy Solidification." (8.37)

Meeting at Tel Aviv University, Tel Aviv, Israel, June 18, 1984

SOLOMON, A. D., "Approximation Methods for Phase Change Problems." (8.17)

SOlOMON, A. D., V. ALEXIADES, AND D. G. WILSON, The Initial Speed of an Emerging Phase Change Front." (8.22) 
9th International CODATA Conference, Jerusalem, Israel, June 24-28, 1984; Proc. The Role of Data in Scientific Progress. P. S. Glaeser (ed.). 1985

MASKEWITZ, B. F. AND J. A. WATTS, ** Overview of the Role of Data for Energy Systems," p. 279. (7.4)

Meeting at Technion-Israel Institute of Technology, Haifa, Israel, June 26, 1984

SOLOMON, A. D., "Mathematical Methods in Latent Heat Storage." (8.18)

Meeting at Ben-Gurion University, Beersheva, Israel, July 2-3, 1984

SOLOMON, A. D., Pitfalls of Computer Simulation." (8.19)

SOlOMON, A. D., G. A. GEIST, AND C. A. SERBIN, "Heat Transfer Considerations in Passive Solar with Energy Storage." (8.2T)

Meeting at the Desert Research Institute, Israel, July 10, 1984

GEIST, G. A., A. D. SOLOMON, C. A. SERBIN, AND J. TOMLINSON, PCMSOLI, A Direct Gain Solar Simulation Code with Phase Change Materials." (8.10)

Gatlinburg 9 international Conference on Numerical Algebra, Waterloo, Ontario, Canada, July II, 1984

FUNDER LIC, R. E., AND R. J. PLEMMONS, ** "Updating LU Factorizations for Computing Stationary Distributions." (8.46)

GILBERT, J. R.,"* AND M. T. HEATH, "Computing a Sparse Basis for the Null Space of a Sparse Matrix."

Society for Industrial and Applied Mathematics National Meeting. University of Washington, Seattle, WA, July 16-20.1984

FUNDERLIC, R. E., AND R. J. PLEMMONS,"* "Updating LU Factorizations for Computing Stationary Distributions." (8.46)

GEORGE, A.,** M. T. HEATH, AND E. NG, "Solution of Sparse Underdetermined Systems of Linear Equations." (8.51)

OSTROUCHOV, G., 'Symbolic Givens Reduction in Large Sparse Least Squares Problems." (8.101)

SERBIN, C. A., "Role of Mathematics and Statistics Research Section of Oak Ridge National Laboratory." (8.15)

ASME Conference on Computers in Engineering, Las Vegas, NV, August 1984

BARHEN, J., C. R. WEISBIN, AND G. de SAUSSURE, "Real-Time Planning by an Intelligent Robot." (4.5)

1984 ASME/AICHE National Heat Transfer Conference, Niagara Falls, NY, August 1984

SOLOMON, A. D., J. J. TOMLINSON,** G. A. GEIST, AND C. A. SER BIN, "Heat Transfer and Phase Change in PCMSOL-I, A Model of a Direct Gain Passive Solar Structure with Latent Heat Storage." (8.28) 
ORNL Colloquium. Oak Ridge, TN. August 13, 1984

BARTINE, D. E., "Developing Nuclear Power for Space Applications." (2.40)

American Statistical Association Annual Meeting, Philadelphia, PA, August 14, 1984; Proc. Section on Survey Research Methods of the American Statistical Association (I984)

TSAO, H.,"* AND T. WRIGHT, "An Empirical Study on Maximizing (or Minimizing) the Number of Retentions in Unequal Probability Sampling Without Replacement: Two Units Per Stratum," p. 218. (8.113)

Passive and Hybrid Solar Energy Update. Washington, DC, September 4-7, 1984

TOMLINSON, J. J.,* G. A. GEIST, AND M. D. MORRIS, "Analysis of Passive Solar Thermal Energy Storage."

Neutron Nucleus Collisions Conference, Glouster, OH, September 5-8, 1984; Proc. AIP Conf. No. $124(1985)$

CARLTON, R. F.,** J. A. HARVEY, AND C. H. JOHNSON,** "Optical Model Scattering Functions for Low Energy Neutrons on ${ }^{86} \mathrm{Kr}, "$ p. 308. (1.29)

CODDENS, G. P,** M. SALAH,** J. A. HARVEY, N. W. HILL, ** "Neutron, Alpha and Total Widths and Spin Assignments for Resonances in ${ }^{33} \mathrm{~S}+n$ from $10-400 \mathrm{keV}, " \mathrm{p}$. 302. (1.25)

HARVEY, J. A., C. H. JOHNSON,** R. F. CARLTON,** AND B. CASTEL** $" d_{s / 2}$-Single Particle Strength in ${ }^{48} \mathrm{Ca}+n$," p. 304. (1.26)

Fifth International Symposium on Capture Gamma-Ray Spectroscopy and Related Topics, Knoxville, TN, September 10-14. 1984; Proc. 1984

BEER, H.,* G. WALTER,** AND R. L. MACKLIN, The ${ }^{163}$ Dy- ${ }^{163}$ Ho Branching: An s-Process Barometer," p. 778. (1.21)

CARLTON, R. F."* J. A. HARVEY, N. W. HILL, AND R. L. MACKLIN, Neutron Capture and Total Cross Sections for ${ }^{48} \mathrm{Ca}$ : Astrophysical Implications," p. 774. (1.10)

FOGELBERG, B.,** J. A. HARVEY, M. MIZUMOTO,** AND S. RAMAN,"* "Observation of Extremely Low $s$-Wave Strength in the Reaction ${ }^{136} \mathrm{Xe}+n, "$ p. 493. (1.33)

Sixth Annual DOE-LLWMP Participants' Information Meeting. Denver, CO, September II-I3, 1984

PIN, F. G., AND E. M. OBLOW, "Application of Pathways Analyses for Site Performance Prediction for the Gas Centrifuge Enrichment Plant and Oak Ridge Central Waste Disposal Facility." (4.31)

Presentation to DOE/OSTI/TIC, Oak Ridge, TN, September 12, 1984

MASKEWITZ, B. F., The Radiation Shieldirg Information Center's Foreign Technology Assessment -- An Important National Asset." (7.2) 
ANS Topical Conference on Reactor Physics and Shielding, Chicago, IL, September 17-19, 1984; Proc. 1984

OZER, 0.,** R. E. MAERKER, M. L. WILlIAMS, ** B. L. BROADHEAD,** J. J. WAGSCHAL,"* AND C. Y. FU, "LEPRICON - A Systematic Approach to LWR Pressure Vessel Dosimetry," Vol. II, p. 674. (4.21)

RHOADES, W. A., R. L. CHILDS,** M. B. EMMETT,** AND S. N. CRAMER, "Applications of the Three-Dimensional Oak Ridge Transport Code," Vol. I, p. 225. (2.14)

SLATER, C. O., AND S. N. CRAMER, "Analysis of Neutron Streaming in the HTGR Lower Support Regions," Vol. II, p. 724. (2.16)

DOE Numerical Anal, sis Special Interest Group Meeting, Argonne National Laboratory, Argonne, IL, September 18-!9, 1984

GEIST, G. A., R. E. FUNDFRLIC, J. MEYERING, ** Some Aspects of a Data Flow Algorithm for Cholesky Decomposition." (8.48)

Fifth ASTM-Euratom Symposium on Reactor Dosimetry, Geesthacht, West Germany, September 24-28, 1984; Proc. Vol. I, Dordrecht. Netherlands (1985)

MAER KER, R. E., AND B. A. WORLEY, "Calculated Spectral Fluences and Dosimeter Activities for the Metallurgical Blind Test Irradiations at the ORR-PSF," p. 187. (4.13)

MAERKER, R. E., B. L. BROADHEAD,** AND M. L. WILlIAMS, ** "Recent Progress and Developments in LWR-PV Calculational Methodology," p. 1639. (4.25)

WAGSCHAL, J. J.,** R. E. MAERKER, B. L. BROADHEAD,** AND M. L. WILLIAMS, ** "Unfolded ANO-1 Fluxes Using the LEPRICON Methodology," p. 1827. (4.20)

1984 Annual Meeting of the Society for Risk Analysis, Knoxville, TN, September 30 - October 3 , 1984

BUCKLEY, J. J.,"* AND V. R. R. UPPULURI, "Fuzzy Hierarchical Analysis." (8.80)

Lectures on Monte Carlo Methods in Nuclear Reactor Analysis, Joint Research Center, Ispra Establishment of the Commission of the European Communitie:, Ispra, Italy, October 8-12, 1984

CRAMER, S. N., AND J. S. TANG,"* "Variance Reduction Methods Applied to DeepPenetration Monte Carlo Problems." (2.4)

CRAMER, S. N., "Applications Guide to the MORSE Monte Carlo Code." (2.3)

CRAMER, S. N., "Applications Guide to the RSIC-Distributed Version of the MCNP Code (Coupled Monte Carlo Neutron-Photon Code)." (2.5)

CRAMER, S. N., "Applications Guide to the RSIC-Distributed Version of the KENO-V Code." (2.6)

Statistics Symposium on National Energy Issues, Seatlle, WA, October 16-18, 1984

BUCKLEY, J. J.," AND V. R. R. UPPULURI, "Fuzzy Hierarchical Analysis." (8.80) 
2lst Annual Meeting of the Society for Engineering Science, Blacksburg, VA, October ,7, 1984

AleXIADES, V., "A New Numerical Method for Phase Change Problems." (8.1)

WILSON, D. G., "Modeling Binary Alloy Solidification." (8.35)

American Physical Society, Nashville, TN, October 18-20, 1984

CARLTON, R. F.,* C. H. JOHNSON,* AND J. A. HARVEY, Total Cross Sections for Low Energy Neutrons on ${ }^{86} \mathrm{Kr}$." (1.30)

Applied Mathematics Colloquium and Nuclear Engineering Seminar, Columbia University, October 22, 1984

CACUCI, D. G., Efficient Sensitivity Analysis of Large Nonlinear Systems with Feedback."

Human Factors Society 28th Annual Meeting on New Frontiers for Science and Technology. San Antonio, TX, October 22-26, 1984

CARTER, R. J., "A Classroom Training Revision Methodology." (5.12)

GRAY, L. H., AND P. M. HAAS, Nuclear Power Plant Control Room Operators Performance Research." (5.10)

JORGENSEN, C. C., "Initial Development of an Automated Task Analysis Profiling System (TAPS)." (5.19)

SIEGEL, A. I.,* W. D. BARTTER,** J. J. WOLF,** H. E. KNEE, AND P. M. HAAS, The Maintenance Personnel Performance Simulation (MAPPS) Model." (5.26)

Twelfth Water Reactor Safety Information Meeting, National Bureau of Standards, Washington, DC, October 22-26. 1984; Proc. NUREG/CP-0058 (1984)

BEARE, A. N."* AND L. H. GRAY, Simulator Experiments: Effects of NPP Operator Experience on Performance," Vol. 6, p. 17. (5.8)

GUTHRIE, V.,* D. J. CAMPBELL,* J. W. CLETCHER,** B. C. ELLISON,** E. J. KIRCHNER,"* H. M. PAULA," R. W. EMMETT," AND G. F. FLANAGAN, "Risk Assessmer: Applicatir :o NRC Inspection," Vol. 6, p.350.

JORGENSEN, C. C., AND R. J. CARTER, "Development of Methods for Nuclear Power Plant Personnel Qualifications and Training," Vol. 6, p. 28. (5.21)

KNEE, H. E., P. M. HAAS, T. G. RYAN,* A. I. SIEGEL,* W. D. BARTTER,* AND J. J. WOLF," "Maintenance Personnel Performance Simulation (MAPPS) Model: Overview and Evaluation Efforts," Vol. 6, p. 61. (5.24)

KROIS, P. A., P. M. HAAS, J. J. MANNING, AND C. R. BOVELL, " "Human Factors Review for Severe Accident Sequence Analysis (SASA)." (Not included in proceedings.) (5.3)

SELBY, D. L., AND J. D. WHITE,* "Integration of PTS Studies to Calculate Throughthe-Wall Crack Probabilities," Vol. 2, p. 1. (6.4)

WHITE, J. E., R. W. ROUSSIN, AND B. F. MASKEWITZ, "NRC Technical Data Management Center (TDMC)." (Poster session, not included in proceedings.) (7.9) 
Southeastern Section Meeting of the American Physical Society. Memphis, TN. October 24-27. 1984

ALSMILLER, F. S., T. A. GABRIEL, AND R. G. ALSMILLER, JR., "Hadron-Lepton Cascade Calculations (1-20 GeV) for a Pb-Al-Lucite Calorimeter." (3.10)

ALSMILLER, R. G., JR., F. S. ALSMILlER, AND T. A. LEWIS,"* "Cakculations Pertaining to the Design of a Prebuncher for a 150-MeV Electron Linear Accelerator: III. Comparisons with Experimental Data." (1.59)

First United States-Japan Workshop on Risk Management, Tsukuba, Japan, October 28-31, 1984

UPPULURI, V. R. R., "Methods in Risk Management - A Working Paper." (8.115)

Symposium on the Radioijology of Radium and Thorotrast, Neuherberg. Federal Republic of Germany. October 29-31. 1984

GROER, P. G.,** AND V. R. R. UPPULURI, "Estimation of the Minimum Latent Period for Radiogenic Osteosarcomas in Man." (8.89)

IEEE 1984 Nuclear Science Symposium, Orlando, FL, October 3I - November 2, 1984

GABRIEL, T. A., B. L. BISHOP,** J. BRAU,** A. Di CIACCIO,** M. GOODMAN,** AND R. WILSON,"* "Compensation Effects in Hadron Calorimeters." (3.9)

HILL, N. W.** J. A. HARVEY, D. J. HOREN,** G. L. MORGAN,** AND R. R. WINTERS, ** "Majority Logic NE-1 10 Detectors for keV Neutrons." (1.56)

International Evaluation of Operational Practices (IEOP) Group Meeting. Paris, France, November 8-9. 1984

HAAS, P. M., "Human Factors Research at Oak Ridge National Laboratory. (5.28)

1984 International Conference of the American Nuclear Society/European Nuclear Society. Washington, DC. November 11-16, 1984; Trans. Am. Nucl. Soc. 47, 1984

DIFILIPPO, F. C., D. G. CACUCI, AND B. A. WORLEY, "Flux Synthesis for the OnLine Surveillance of Nuclear Power Plants," p. 441. (2.19)

De SAUSSURE, G., R. B. PEREZ, AND ROGER MACKLIN,"* "Multilevel Analysis of the Low-Energy ${ }^{239}$ Pu Cross Sections," p. 428. (1.41)

KING, T. L." R. B. PEREZ, D. G. CACUCI, R. T. WOOD,* AND E. L. MACHADO, * "Application of Variational-Heuristic Learning Methods to Reactor Physics: A New Concept in Artificial Intelligence," p. 439. (2.25)

Advisory Group Meeting on Nuclear Standard Reference Data. Central Bureau for Nuclear Measurements, Geel, Belgium. November 12-18, 1984

CARLSON, A. D.,* W. P. POENITZ,** G. M. HALE,** AND R. W. PEElle, The Neutron Cross Section Standards Evaluations for ENDF/B-VI." (1.47)

Annual Meeting of the American Civil Defense Association, Daytona Bearh, FL, November 15-16, 1984

HAALAND, C. M., "In the Shadow of Ground Zero." (2.47)

THURMER, R N., "Improving RADEF." (2.54) 
Annual Meeting of the Tennessee Academy of Sciences, Mathematics Section, Knoxville, TN. November 16-17, 1984

McHENRY, C. E.,** S. A. PATIL,** AND V. R. R. UPPULURI, "First Digits of Exponentially Distributed Random Variables." (8.93)

International Conference for Power Plant Simulation, Cuernavaca, Morelos, Mexico, November $19-21,1984$

THOMPSON, S., AND P. G. TUTTLE,** "Event Detection in Continuous Simulation." (8.31)

First Conference on Artificial Intelligence Applications, Denver. CO. December 5-7, 1984; Proc. (1985)

WEISBIN, C. R., G. de SAUSSURE, J. BARHEN, E. M. OblOW, AND J. C. WHITE,"* "Minimal Cut-Set Methodology for Artificial Intelligence Applications," p. 465. (4.3)

23rd IEEE Conference on Decision and Control, Las Vegas, NV, December 12-14, 1984; Proc. Vol. 2. 1984

HEATH, M. T., "Sparse Matrix Computations," p. 662. (8.59)

LEP Experimenters' Workshop on Shower Simulation, CERN, Geneva, Switzerland, January $29-31.1985$

GA RIEL, T. A., The High-Energy Transport Code, HETC." (3.8)

GABRIEL, T. A., AND M. B. EMMETT,** "An Introduction to Combinatorial Geometry." (3.14)

L.ecture, Department of Industrial Engineering. University of Tennessee, Knoxville, TN, February 1985

HAAS, P. M., "Human Factors Research at Oak Ridge National Laboratory."

Seventh Annual Symposium on Management of Uranium Mill Tailings, Low-Level Waste and Hazardous Waste, Colorado State University, Fort Collins, CO, February 6-8, 1985

PIN, F. G., AND E. M. OBLOW, "Pathways Analyses and Their Role in the Decision Making Process for Selection of Low-Level Waste Disposal Sites." (4.32)

Workshop on the Application of a Minirn. puter for Large-Scale Nuclear Computation, NRC, Washington, DC, February 7-8, 1985

WHITE, J. E., AND R. W. ROUSSIN, User's Guide for the NRC Staff Version of CRAC2." (7.10)

Workshop on Efficient Data Collection, University of California, Berkeley, CA, February 11-13, 1985

MITCHELL, T. J., "Compute, Construction of Supersaturated Screening Designs." (8.95) 
1985 WATTec Conference, Knoxville, TN, February 12, 1985

HAALAND, C. M., "A Review of the Nuclear Winter Scenario." (2.49)

HALL, M. C. G., "Carbon Dioxide Build ıp and Climate: Potential Impacts." (4.35)

WEISBIN, C. R., I. BARHEN, G. de SAUSSURE, IV. R. HAMEL,* C. JORGENSEN, J. L. LUCIUS, ** E. M. OBLOW, T. SWIFT,"* "Artificial Intelligence and Concurrent Computation for Robotic Applications." (4.1)

Sixth Topical Meeting on the Technology of Fusion Energy, San Francisco, CA, March 3-7, 1985

R. E. MacFARLANE, ** R. W. ROUSSIN, AND R. J. HOWERTON,** Status of Data Processing Codes and Data Libraries."

SANTORO, R. T., J. M. BARNES,** R. G. ALSMILLER, JR., AND J. D. DRISCHLER, "Monte Carlo and Discrete Ordinates Calculations of 14-MeV Neutrons Streaming Through a Stainless Steel Duct: Comparison with Experiment." (3.1)

SANTORO, R. T., N. A. UCKAN,** AND R. J. SCHMITT,** "Magnetics Calculations for an ELMO Bumpy Square." (3.5)

1985 Eastern Simulation Conference, Norfolk, VA, March 3-8, 1985

CARTER, R. J., AND K. R. LAUGHERY,** "A Methodology for the Evaluation of Nuclear Power Plant Simulation Facilities." (5.14)

Ellis R. Ott Conference, New Brunswick, NJ, March 18-21, 1985

SCHLOTZHAUER, D. C., "Software Systems for Statistical Process Control and Simulated-Process Optimization." (8.103)

Meeting at the University of Tennessee at Chattanooga, Chattanooga, TN, March 22, 1985

WILSON, D. G., "Mathematical Modeling — Fun and Games with Applications." (8.34)

IEEE Workshop on Special Computer Architectures for Robotics and Automation, International Conference on Robotics Research, St. Louis, MO, March 25, 1785

BARHEN, J., "Hypercube Concurrent Computation and Virtual Time for Robotic Application." (4.8)

Meeting at the University of Miami, Coral Gables, FL, April 5, 1985

WILSON, D. G., "Computer Modeling of Solidification Processes." (8.36)

1985 Eastern Regional Student Conference, Gainesville, FL. April 5-6, 1985

TIMMERMAN, R. D., "Assessing Applications of Sauer's Method for Calculating Dancoff Factors." (2.44)

Parallel Computing Seminar, Mathematics Department. North Carolina State University. Raleigh. NC, April 8 12, 1985

FUNDERLIC, R. E., AND G. A. GEIST, The Hypercube Architecture and Some Aspects of Data Flow for Parallel Computation of Missized Matrix Problems." (8.42) 
International Meeting on Advances in Nuclear Engineering Computaiton Methods, Knoxville, TN. April 9-11, 1985: Proc. (1985)

CRAMER, S. N., J. GONNORD,** AND J. S. HENDRICKS, ** "Monte Carlo Techniques for Analyzing Deep-Penetration Problems," Vel. 2, p. 812. (2.7)

FRANK, B. R.,** AND J. R. WHITE,"* "Fen-Group Three-Dimensional Hexagonal Geometry HTGR Benchmark Problem," Vol. 1, p. 194. (2.43)

VONDY, D. R., Solution of a Two-Dimensional PWR Benchmark Problem Modeling Two Cycles with Refueling and Fuel Assembly Repositioning by Finite-Difference Method," Vol. I, p. 159 (2.33)

Meeting at the NATO Advanced Study Institute, on Scaling Phenomena in Disordered Systems, Geilo. Norway, April 10-21, 1985

GRAY, L. J., S. H. LIU ** AND T. KAPLAN,"* "A Fractal Model for Charge Diffusion Across A Rough Interface." (8.12)

1985 Conference on Intelligent Systems and Machines, Oai land University, Rochester, MI, April 22-24, 1985

WEISBIN, C. R., J. BARHEN, G. de SAUSSURE, W. R. HAMEL,** C. C. JORGENSEN, E. M. OBLOW, AND R. E. RICKS, "Machine Intelligence for Robotics Applications." (4.6)

Society for Industrial and Applied Mathematics Conference on Applied Linear Algebra, Raleigh, NC. April 29 - May 2, 1985

FUNDERLIC, R. E., AND C. D. MEYER, JR.,"* "Sensitivity of the Stationary Distribution Vector for an Ergodic Markov Chain." (8.44)

GEIST, G. A., AND R. E. FUNDERLIC, "Torus Data Flow for Parallel Computation of Missized Matrix Problems." (8.43)

HEATH, M. T., "Some Sparse Matrix Problems in Structural Analysis." (8.58)

Workshop on Alloy Solidification, Florence, Italy, May 1985

ALEXIADES, V., "The ORNL Binary Alloy Solidification Model." (8.4)

JASPER Working Group Meeting, Tokyo, Japan, May 7-9, 1985

INGERSOLL, D. T., "Oak Ridge National Laboratory Tower Shielding Facility."

INGERSOLL, D. T., "Description of Proposed JASPER Work Scope."

53rd Statistical Mechanics Meeting, Rutgers University, May 9-10, 1985

PROTOPOPESCU, V., W. KLEIN,"* AND T. KEYES,"* "Time-Dependent Transport as Critical Phenomenon." (2.56)

International Workshop on Expert Systems and Their Applications - Palais des Congres Avignon. France, May 13 15. 1285

OBLOW, E. M., "A Hybr.t Uncertainty Theory." (4.11) 
International Atomic Energy Agency Specialist's Meeting on Safety and Accident Analysis for Gas-Cooled Reactors, Oak Ridge, TN, May 13-15, 1985

MOSES, D. L., AND W. D. LANNING,** The Analysis and Evaluation of Recent Operational Experience from the Fort St. Vrain HTGR." (2.45)

International Conference on Nuclear Data for Basic and Applied Sciences, Santa Fe, NM, May $13-17,1985$

CARLSON, A. D.,** W. P. POENITZ,** G. M. HALE,** AND R. W. PEELLE, The Neutron Cross Section Standards Evaluations for ENDF/B-VI." (1.47)

de SAUSSURE, G., R. B. PEREZ, AND ROGER L. MACKLIN,"* 'R-Matrix Analysis of the ${ }^{239} \mathrm{Pu}$ Neutron Cross Sections." (1.12)

DIFILIPPO, F. C., B. A. WORLEY, AND D. R. VONDY, Neutronic Calculations for a New High Flux Reactor." (2.20)

FU, C. Y., Pairing Correction of Particle-Hole State Densities for Two Kinds of Fermions." (1.39)

GWIN, R., R. R. SPENCER, R. W. INGLE, *" "Measurement of the Energy Dependence of Prompt Neutron Emission from ${ }^{233} \mathrm{U},{ }^{235} \mathrm{U}$, and ${ }^{239} \mathrm{Pu}$ for $E_{n}=0.0005$ to $10 \mathrm{MeV}$ Relative to Emission from Spontaneous Fission of ${ }^{252} \mathrm{Cf}$." (1.4)

HETRICK, D. M.,"* C. Y. FU, AND D. C. LARSON, "Calculated Neutron-Induced Cross Sections for ${ }^{63.65} \mathrm{Cu},{ }^{58.60} \mathrm{Ni}$, and ${ }^{52} \mathrm{Cr}$ from i to $20 \mathrm{MeV}$ and Comparisons with Experime.its." (1.38)

LARSON, D. C., "High Resolution Structural Material $(n, x \gamma)$ Production Cross Sections for $E_{n}$ from 0.2 to $40 \mathrm{MeV}$." (1.1)

LARSON, N. M.,** "Analysis of Neutron Data in the Resonance Region Via the Computer Code SAMMY." (1.54)

LARSON, N. M.,** "Uncertainty Propagation from Raw Data to Final Results." (1.52)

MAERKER, R. E., B. L. BROADHEAD,** C. Y. FU, J. J. WAGSCHAL,** J. WILLIAMS,** AND M. L. WILLIAMS,** "Combining Integral and Differential Dosimetry Data in an Unfolding Procedure with Application to the Arkansas Nuclear One-Unit 1 Reartor." (4.22)

PEELLE, R. W., "Summary Talk Covering Application-Oriented Sessions." (1.51)

PEREY, C. M., F. G. PEREY, J. A. HARVEY, N. W. HILl,** AND R. L. MACKL.IN, ${ }^{n s 8} \mathrm{Ni}+n$ Transmission, Capture and Differential Elastic Scattering Data Analysis is the Resonance Region." (1.11)

PEREY, F. G., "Status of the Parameters of the 1.15-keV Resonance of ${ }^{56} \mathrm{Fe}$." (1.46)

ROUSSIN, R. W., J. E. WHITE, AND J. L. BARTLEY, VITAMIN-E $174 n, 38 g$ General Purpose Cross Section Library." (7.5)

SAlAH, M. M.," J. A. HARVEY, N. W. HILl,"* A. Z. HUSSEIN,"* AND F. G. PEREY, "Accurate Determination of the Parameters of the 292.4-eV Resonance of ${ }^{91} \mathrm{Zr}$ and the 301.3-eV Resonana i ${ }^{96} \mathrm{Zr} . "$ (1.31) 
SPENCER, R. R., J. A. HARVEY, N. W. HILL,** AND L. W. WESTON, Neutron Total Cross Section of Pu-240 Below $6 \mathrm{eV}$ and the Parameters of the 1.056-eV Resonance." (1.35)

WESTON, L. W., AND J. H. TODD,** Capture in the 1.15-keV Iron Resonance." (1.17)

Cincast Service Union AG, Zurich, Switzerland, May 14, 1985

WILSON, D. G., "Computer Modeling of Solidification Processes." (8.36)

Seminars at the University of Torino, Italy, the University of Crete. Greece, and the University of Thessaloniki, Greece, May 16-28, 1985

ALEXIADES, V., "Remarks and Questicns Abrut Hyperbolic Stefan Problems." (8.3)

ALEXIADES, V., "Alloy Solidification with Constitutional Supercooling." (8.2)

ALEXIADES, V., "Minimizers of the Free Energy in the Thermodynamic Theory of Fluid Interfaces." (8.5)

Universily of Tokyo, Tokyo, Japan, May 20, 1985

BOWMAN, K. O., "Approximations to Distributions." (8.70)

Talk presented at Oakland University, Rochester, MI, May 22, 1985

SOLOMON, A. D., "What Is Mathematics to Us?" (8.21)

Presentation to DOE International Affairs, Washington, DC, May 24, 1985

MASKEWITZ, B. F., The Radiation Shielding Information Center's Foreign Technology Assessment - An Important National Asset." (7.2)

Robots 9 Conference, Detroit, MI. June 2-6, 1985

WEISBIN, C. R., J. BARHEN, G. de SAUSSURE, W. R. HAMEL,* C. JORGENSEN, j. L. LUCIUS,"* E. M. OBLOW AND T. SWIFT,** "HERMIES-1: A Mobile Robot for Navigation and Manipulation Experiments." (4.7)

Lecture at the International Workshop on Operator Theory and Its Applications, Amsterdam, Holland, June 3-7, 1985

PROTOPOPESCU, V., The Abstract Time-Dependent Transport Problem." (2.63)

1985 Annual Meeting of the American Nuclear Society, Boston. MA, June 9-14. 1985; Trans. Am. Nucl. Soc. 49 (1985)

FRANK, B. R.,* AND J. R. WHITE,** "Evaluation of the DIF3D Nodal Option for HTGR Neutronic Applications," p. 436. (2.42)

MAERKER, R. E., B. L. BROADHEAD,*" AND M. L. WILLIAMS,"* "Application of LEPRICON Methodology to the Unfolding of Neutron Fluxes in the ANO-1 Reactor," $p$. 425. (4.23)

PEELLE, R. W., "Improving Neutron Reaction Data Through Linac-Based Flight-Time Spectrometry," p. 206. (1.50) 
WEISBIN, C. R., J. BARHEN, G. de SAUSSURE, W. R. HAMEL,** C. JORGENSEN, J. L. LUCIUS,** E. M. OBLOW, AND T. SWIFT,** "Artificial Intelligence and Concurrent Computation for Robotic Applications," p. 310. (4.1)

WHITE, J. R.,** T. J. CAHILL,** B. R. FRANK,** "Exact Analytic Solution for the Generalized Flux in One-Dimensional Cartesian Geometry," p. 220. (241)

WORLEY, B. A., F. C. DIFILIPPO, AND D. R. VONDY, Thermal Reactor Design for a Neutron-Scattering Source Facility." p. 422. (2.22)

Lecture at the $I X^{\text {th }}$ International Conference on Transport Theory, Montecatini, Italy, June 10-14, 1985.

PROTOPOPESCU, V., "A Survey of Recent Results and Open Problems in TimeDependent Transport Theory." (264)

RSIC MORSE-CGA Workshop, Oak Ridge, TN, June 17-20, 1985

EMMETT, M. B.,"* S. N. CRAMER, AND J. S. TANG,** "Basic MORSE Concepts and Basic Input/Output."

EMMETT, M. B.," S. N. CRAMER, AND J. S. TANG,** "MARS Geometry and User Routines."

EMMETT, M. B.,** S. N. CRAMER, AND J. S. TANG,"* "JUNEBUG-Ii."

EMMETT, M. B.,"* S. N. CRAMER, J. S. TANG, ** AND R. W. ROUSSIN, Special MORSE Topics and Features."

1985 IEEE Third Conference on Human Factors and Power Plants. Monterey, CA, June 23-27. 1985

HAAS, P. M., Training Related Research and Levelopmeni Conducted at Oak Ridge National Laboratory for the U.S. Nuclear Regulatory Commission." (5.15)

HAAS, P. M., R. J. CARTER, AND K. R. LAUGheRY,** "Nuclear Powet Plant Simulation Facility Evaluation Methodology." (5.16)

KROIS, P. A., AND P. M. HAAS, "Collection and Analysis of Training Simulator Data." (5.11)

International Conference on Nuclear Power Plant Aging, Availability Factor and Reliability Analysis, San Diego, CA. July 8-I2, 1985

KNEE, H. E., The Maintenance Personnel Performance Simulation (MAPPS) Model: A Human Reliability Analysis Tool." (5.23)

PAINTER, S. L., M. D. ANDERSON, AND H. E. KNEE, "CREDO Data Analysis: A Comparison of Sodium and Water Valve Reliabilities." (5.5)

Summer Conference of the Sociely for Computer Simulation, Chicago, IL, July 22-24, 1985

THOMPSON, S., "A Device Which Allows Special Event Detection in Continuous Simulation Languages without Discrete Blocks." (8.30) 
Fifth International Conference on Mathematical Modeling, University of California, B̈erkete;, CA: July 29, 1985

Flanagan, G., AND V. R. R. UPPULURI, Prioritization of a Large Number of R\&D Programs." (6.5)

1985 ASME International Conference on Computers in Engineering. Boston, MA. August 4-8. 1985

BARHEN, J., "Robot Inverse Dynamics on a Concurrent Computation Ensemble." (4.9)

FORREST-BARLACH, M. G.,"* S. M. BABCOCK,"* H. SINGH,** AND M. J. RABINS,** "Control of a Single Link, Two-Degree-of-Freedom Manipulator with Joint Compliance and Actuator Dynamics." (4.10)

Annual Joint Meeting of the American Statistical Association, Institute of Mathematical Statistics Biometric Society, Las Vegas, NV. August 5-8, 1985

BOWMAN, K. O., AND L. R. SHENTON,** The Distribution of Moment Estimators for Parameters of the Generalized Poisson Distribution." (8.74)

FROME, E. L., AND D. L. CRAGLE ** "Application of Poisson Regression to the Analysis of Childhood Leukemia Deaths in Utah." (8.85)

OSTROUCHOV, G., "ANOVA Model Fitting via Sparse Matrix Computations." (8.99)

TShO, H.,** AND T. WRIGHT, "An Empirical Study on the Stabilities of Difference Estimators." (8.112)

American Nuclear Society Topical Meeting on Compu.er Applications for Nuclear Power Plant Operation and Control. Pasco. WA. September 8-12, 1985

CAMPBELl, D. J.** B. C. ELLISON,** J. C. GLYNN,** AND G. F. FlaNAGAN, "PRISIM: A PC Program to Display Risk Status Information." (6.1)

International Topical Meeting on High-Level Waste Disposal Engineering and Technology, Pasco. WA, September 24-26, 1985

PIN, F. G., B. A. WORLEY, E. M. OBLOW, R. Q. WRIGHT,** AND W. V. HARPER," "Automated Sensitivity Analysis of the Radinnuclide Migration Code UCB.NE-10.2." (4.33)

29th Human Factors Society Meeting, Baltimore, MD, September 29-October 3, 1985

CARTER, R. J, AND K. R. LAUGHERY,** Nuclear Power Plant Training Simulator Fidelity Assessment." (5.13)

KROIS, P. A., AND P. M. HAAS, "Human Factors Review for Nuclear Powes Plant Severe Accident Sequence An slysis." (5.2)

1985 Annual Meeting of the American Nuclear Society, San Francisco, CA, November 10-14. 1985; Trans. Am. Nucl. Soc. 50 (1985;

CHILDS, R. L.," AND W. A. RHOADES, The Extension of the Linear Nodal Method to Large Concrete Building Calcrlations," p. A76. (2.10) 


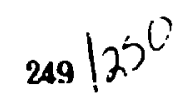

FU, C. Y., AND D. T. INGERSOLL, "Impact of ${ }^{57} \mathrm{Fe}$ on Neutron Penetration in Thick Sodium-Iron Shields," p. 470. (2.11)

MAERKER, R. E., AND B. L. BROADHEAD, "Correlations Between Calculated Surveillance Dosimeter Activities and Pressure Vessel Fluxes in the Arkansas Nuclear One-Unit I Reactor," p. 436. (4.27)

RHOADES, W. A., R. L. CHILDS,** D. T. INGERSOLL, AND F. J. MUCKENTHALER, "Analysis of the TORT Validation Experiment," p. 473. (2.15)

THOMPSON, J. L.,* M. B. EMMETT,** W. A. RHOADES, AND H. L. DODDS, JR.,"* "A Method for Coupling Two-Dimensional to Three-Dimensional Discrete Ordinates Calculations," p. 475. (2.8)

Second Society for Industrial and Applied Mathematics Conference on Parallel Processing for Scicntific Computing, Norfolk, VA, November 20, 1985

WILSON, D. G., Vectorization of Multidimensional Finite Difference Schemes with Application to Stefan-Type Problems." (8.33) 


\section{AUTHOR INDEX}

Aifantis, E. C. 8.7

Alexiades, V. 8.1, 8.2, 8.3, 8.4, 8.5, 8.6,

$8.7,8.8,8.9,8.22,8.23,8.24,8.25,8.26$,

8.29, 8.37, 8.38

Allisen, L. J. 7.12

Alsmiller, F. S. $\quad 1.59,3.10$

Alsmiller, R. G., Jr. $\quad 1.59,3.1,3.2,3.3,3.7$, $3.10,3.15$

.Inderson, M. D. $5.1,5.5$

Apostolakis, G. 6.3

Agrawal, H. M. 1.28

Auchampaugh, G. F. 1.7

Babcock, S. M. 4.10

Barhen, J. 4.1, 4.3, 4.4, 4.5, 4.6, 4.7, $4.8,4.9$

Barnes, J. M. 3.1, 3.2, 3.3, 3.6, 3.7

Bartine, D. E. $2.12,2.40$

Bartley, J. L. 7.5, 7.6

Bartter, W. D. 5.24, 5.25, 5.26, 5.27

Bate, L. C. 8.81

Bayne, C. K. 8.65

Beals, R. 2.62

Beare, A. N. $5.6,5.7,5.8$

Beauchamp, J. J. $8.65,8.66,8.67,8.107$

Beer, H. 1.20, 1.21, 1.22

Berley, D. 3.12, 3.13

Bernard, S. R. 8.77, 8.78, 8.109, 8.110

Berry, M. W. $8.39,8.40$
Bishop, B. L. $3.9,3.11,3.12,3.13$

Block. R. C. 1.8

Bovell, C. R. 5.3

Bowman, K. O. 8.41, 8.68, 8.69, 8.70, 8.71, $8.72,8.73,8.74,8.75,8.76,8.77,8.78$, 8.79, 8.:08

Braden, P. W. 8.88

Brau, J. 3.9, 3.11

Broadhead, B. L. $2.46,4.17,4.18,4.20,4.21$, $4.22,4.23,4.24,4.25,4.26,4.27$

Buckley, J. J. 8.80

Cacheiro, N. L. A. $\quad 8.87,8.88$

Cacuci, D. G. $2.19,2.25,2.26,2.27,2.28$, $2.29,2.30,2.31,2.46,4.38$

Cahill, T. J. 2.41

Cain, K. T. $\quad 8.87,8.88$

Calle, E. E. 8.120

Campbell, D. J. $\mathrm{FI}$

Cannon, J. B. 4.30

Carlson, A. D. 1.47

Carlton, R. F. 1.9, 1.10, 1.24, 1.26, 1.27, I.29, 1.30

Carter, B. J. 7.3

Carter, R. J. $5.12,5.13,5.14,5.16,5.21$

Castel, B. 1.9, 1.26, 1.27

Chapman, G. T. 3.7

Checkoway, H. 8.84

Childs, R. L. $2.9,2.10,2.14,2.15$

Christie, N. T, 8.81 
Coddens, G. P. 1.25

Cole, R. G. $\quad 2.60,2.61$

Cornett, C. V. $\quad \mathbf{8 . 8 7}, 8.88$

Cragle, D. L. 8.85

Cramer, S. N. 2.3, 2.4, 2.5, 2.6, 2.7, 2.14, 2.16

Dabbs, J. W. T. 1.8

Dalbey, W. 8.92

de Saussure, G. $1.41,1.42,1.43,4.1,4.3$, $4.4,4.5,4.6,4.7$

Di Ciaccio, A. 3.9

Dickens, J. K. 1.2, 1.57

Dietrich, W. C. 8.13

Difilippo, F. C. $2.18,2.19,2.20,2.21,2.22$

Dodds, H. L., Jr. 2.8

Donovan, M. D. 5.6

Dorris, R. E. $\quad 5.7$

Dougan, R. J. $\quad 1.8$

Drake, J. 8.26

Driemeyer, D. E. 3.6

Drischler, J. D. $3.1,3.3,3.4$

Ebey, S. F. $\quad 8.97,8.98$

Eich, W. J. 4.28

El-Bassioni, A. A. 6.2

Ellison, B. C. 6.1

Ellsworth, R. W. $3.12,3.13$

Enunct, M. B. $2.1,2.2,2.8,2.14,3.14$

Engle, W. W., Jr. 2.12

Extermann, R. C. $\quad 1.7$

Flanagan, G. F. 6.1, 6.5

Fogelherg, B. 1.33, 1.34

Ford, W. F., III 4.19

Forrest-Ba-lach, M. G. 4.10
Frank, B. R. 2.41, 2.42, 2.43

Freudenreich, H. T. $3.12,3.13$

Frome, E. L. $\quad 8.82,8.83,8.84,8.85,8.86$, 8.90

Fu, C. Y. $1.37,1.38,1.39,1.40,2.11$, $4.18,4.21,4.22$

Funderlic, R. E. $\quad 8.42,8.43,8.44,8.45$, 8.46, 8.48

Fussell, J. B. 5.4

Gabriel, T. A. 3.8, 3.9, 3.10, 3.11, 3.12, $3.13,3.14$

Gaffney, P. W. 8.47

Garg, J. B. 1.28

Geist, G. A. $8.8,8.10,8.27,8.28,8.42$, $8.43,8.48,8.49$

Generoso, W. M. $\quad 8.87,8.88$

George, A. $8.50,8.51,8.52,8.53,8.54$, 8.55

Glover, P. L. 8.102

Glynn, J. C. 6.1

Gonnord, J. 2.7

Good, W. M. 1.9

Goodman, J. A. $3.12,3.13$

Goodman, M. 3.9

Gopinath, D. V. 7.11

Gosslee, D. G. $\quad 8.81,8.87,8.88,8.10^{2} 2$

Gray, L. H. $\quad 5.6,5.7,5.8,5.9,5.10$

Gray, L. J. $8.11,8.12,8.13,8.14$

Griesemer, R. 8.92

Groer, P. G. $\quad 8.89$

Gwin, R. 1.3, 1.4, 1.5

Haaland, C. M. $2.47,2.48,2.49,2.50,2.51$, $2.52,2.53$

Haas, P. M. 5.1, 5.2, 5.3, 5.4, 5.9, 5.10, $5.11,5.15,5.16,5.17,5.24,5.26,5.27$, 5.28 
Haas. S. M. 3.15

Haight, R. C. 1.49

Hale, G. M. 1.47

Hall, M. C. G. 4.35, 4.36. 4.37, 4.38

Hamel, W. R. 4.1, 4.6, 4.7

Hargis, J. 3.11

Harper, W. V. 4.33

Harris, D. R. 1.8

Harvey, J. A. 1.7, 1.9, 1.10, 1.11, 1.12, 1.24, $1.25,1.26,1.27,1.28,1.29,1.30,1.31,1.32$, $1.33 .1 .34,1.35,1.56,1.60,1.61$

Heath, M. T. $8.39,8.40,8.49,8.50,8.51,8.56$, $8.57,8.58,8.59,8.60,8.61,8.62,8.64$

Hendricks, J. S. 2.7

Hetrick, D. M. 1.37, 1.38

Hill, N. W. 1.7, 1.10, 1.11, 1.12, 1.25, 1.31, $1.32,1.35,1.56,1.57$

Hoff, R. W. 1.8

Holland, J. M. 8.90

Horen, D. J. $1.12,1.56,1.58$

Horwedel, J. E. 3.15

Hou, F. S. $\quad 1.57$

Hughes, L. A. 8.88

Hussein, A. Z. I.31

Ingersoll, D. T. 2.11, 2.15

Ingle, R. W. 1.3, 1.4, 1.5

Jacohs, B. F. 7.12

Jacobson, K. B. 8.81

Janardan, K. (j. 8.91

Johnson, C. H. $1.24,1.26,1.27,1.29,1.30$

Jorgensen, C. C. 4.1, 4.6, 4.7, 5.17, 5.18, $5.19,5.20,5.21$

Kaizerman, S. 2.26, 2.27, 2.28

Kam, F. B. K. 4.16
Kane, V. E. $8.65,8.66$

Kaneko, I. 8.39

Kaplan, T. 8.12,8.14

Ketelle, R. H. $\quad 4.30$

Keyes, T. $2.56,2.57,2.60,2.6 \mathrm{I}$

King, T. L. 2.25

Kirk, B. L. $\quad 7.7,7.8$

Klaus, M. 2.59

Klein, W. 2.56

Knee, H. E. $5.1,5.4,5.5,5.23,5.24,5.25$, $5.26,5.27$

Krois, P. A. 5.2, 5.3, 5.4, 5.11

Kumar, K. D. 8.67

Lam, H. K. $8.71,8.72,8.73$

Lanning, W. D. 2.45

Larson, D. C. I.I, 1.2, 1.37, 1.38, 1.48, $1.49,1.60,1.61$

Larson, N. M. 1.43, 1.52, 1.53, 1.54, 1.55, $1.60,1.61$

Lassiter, D. L. 5.6

Laub. A. J. 8.60

Laughery, K. R. $\quad 5.13,5.14,5.16,5.22$

Lawo, M. 8.39

Lee, D. W. 4.30

Lewis, T. A. 1.59

Liu, J. W. H. $8.50,8.52,8.63$

Liu, S. H. 8.12

Lock, S. 8.92

Lougheed, R. W. $\quad 1.8$

Lowry, J. C. $\quad 5.9,5.17$

L.ucius, J. L. 4.1, 4.7

Machado, E. L. 2.25

Macklin, R. I.. 1.9, 1.10, I.II, 1.12,1.13, $1.14,1.15,1.16,1.18,1.19,1.20,1.21$. $1.22,1.32$ 
Macklin, Roger L. $1.41,1.42,1.43$

Maerker, R. E. $\quad 4.12,4.13,4.14,4.15,4.16$, $4.17,4.18,4.19,4.20,4.21,4.22,4.23$, $4.24,4.25,4.26,4.27$

Maguire, H. T., Jr. 1.8

Manneschmidt, J. F. 3.2

Manning, J. J. 5.3, 5.4, 5.9

March-Leuba, J. 2.29, 2.30, 2.31, 2.32

Marshalla, R. A. 3.15

Martin, J. A. 1.58

Maskewitz, B. F. 7.1, 7.2, 7.3, 7.4, 7.9, 7.12

McCarthy, J. F. 8.107

McConnell, J. W. 1.57

Mclain, R. 8.86

McHenry, C. E. 8.93

McKechan, K. 4.2

McMahan, C. A. 8.94

McSweeny, P. S. 8.120

Merriman, L. D. 1.36

Meyer, C. D, Jr. 8.44

Meyering, J, 8.48

Mincer, A. I. $3.12,3.13$

Mitchell, T. J. $8.95,8.111,8.114$

Mizumoto, M. $1.33,1.34$

Montague, D. F. 5.4

Moore, M. S. 1.7

Morelock, T. C. 5.4

Morgan, G. L. 1.56

Morris, M. D. $8.94,8.96,8.97,8.98$, 8.120

Moses, D. L. 2.45

Moses, J. D. 1.7

Muckenthaler, F. J. 2.13, 2.15
Mughabghab, S. F. $\quad 1.13$

Natarajan, A. 7.11

Nelson, R. O. 1.7

Nesbitt, D. M. 3.15

Nestor, C. W., Jr. 8.110

$\mathrm{Ng}$, E. $\quad 8.51,8.53,8.54,8.55$

Oblow, E. M. 4.1, 4.3, 4.6, 4.7, 4.11, 4.31, $4.32,4.33,4.34$

Olsen, C. E. 1.7

Olsen, D. K. $1.44,1.45,1.58$

Ostrouchov, G. $8.99,8.100,8.101$

Ozer, $0 . \quad 4.21,4.28$

Pace, J. V., III 2.46

Paige, C. C. 8.60

Painter, S. L. 5.1, 5.5

Patchett, P. J. 1.20

Patil, S. A. $8.93,8.116$

Peelle, R. W. $1.47,1.50,1.51$

Perey, C. M. I.11

Perey, F. G. $1.11,1.31,1.46,1.61$

Perez, R. B. $1.41,1.42,1.43,2.25,2.29$, $2.30,2.31,2.32$

Perkins, E. H. 8.102

Pfuderer, H. A. 7.12

Pierce, D. E. 1.61

Pin, F. G. $4.30,4.31,4.32,4.33$

Plemmons, R. J. $\quad 8.39,8.40,8.45,8.46,8.62$

Poenitz, W. P. 1.47

Protopopescu, V. 2.55, 2.56, 2.57, 2.58, 2.59, $2.60,2.61,2.62,2.63,2.64$

Rabins, M. J. 4.10

Raman, S. $1.33,1.34$

Rhoades, W. A. $2.8,2.9,2.10,2.14,2.15$ 
Rhyne, W. Q. 6.2

Ricks, R. E. 4.6

Roussin, R. W. 7.1, 7.5, 7.6, 7.9, 7.10

Ryan, T. G. 5.24

Salah, M. $1.25,1.31$

Santoro, R. T. 3.1, 3.2, 3.3, 3.4, 3.5, $3.6,3.7$

Savage, M. G. $2.23,2.24$

Schlotzhauer, D. C. 8.103

Schmitt, R. J. 3.5

Schmoyer, R. L. $\quad 8.92,8.104,8.105,8.106,8.107$

Schuknecht, B. A. $\mathbf{8 . 1 2 0}$

Scoles, S. W. 1.5

Scott, D. S. 8.64

Seals, R. H. 1.61

Selby, D. L. $5.17,6.4$

Serbin, C. $\quad 8.10,8.13,8.15,8.27,8.28$

Shenton, L. R. 8.71, 8.72, 8.73, 8.74, 8.75, $8.76,8.77,8.78,8.79,8.108$

Siegel, A. I. $5.24,5.25,5.26,5.27$

Singh, H. $\quad 4.10$

Siu, N. 6.3

Slater, C. O. $2.16,2.17$

Slovacek, R. E. $\quad 1.8$

Sobel, M. $8.109,8.110$

Solomon, A. D. $\quad 8.6,8.8,8.9,8.10,8.16$, $8.17,8.18,8.19,8.20,8.21,8.22,8.23$, $8.24,8.25,8.26,8.27,8.28,8.29,8.37$, 8.38

Soran, P. D. 3.7

Sorensen, D. C. 8.61

Spencer, R. R. $1.3,1.4,1.5,1.35,1.57$

Stallman, F. W. 4.16

Stopa, C. R. $\quad 1.8$
Storer, J. B. 8.111

Subbaiah, K. V. 7.11

Sweeney, F. J. 2.32

Swift, T. E. $4.1,4.7$

Szekeres, G. 8.79

Tang, J. S. $2.4,3.7$

Thompson, J. L. 2.8

Thompson, S. $8.30,8.31$

Thurmer, R. N. 2.54

Timmerman, R. D. 2.44

Todd, J. H. $\quad 1.5,1.6,1.17,1.23$

Tomerian, N. $2.26,2.27,2.28$

Tomlinson, J. $\quad 8.10,8.28$

Tonwar, S. C. $3.12,3.13$

Trubey, D. K. 7.1, 7.11

Tsang, F. Y. 1.57

Tsao, H. $8.112,8.113,8.118,8.119$

Turnbull, B. W. 8.114

Tuttle, P. G. 8.31

Uckan, N. A. $3.5,3.6$

Uppuluri, V. R. R. $\quad 6.5,8.80,8.89,8.91,8.93$, $8.109,8.110,8.115,8.116$

van der Mee, C. V. M. 2.58, 2.59

Vondy, D. R. 2.20, 2.22, 2.33, 2.34, 2.35, $2.36,2.37,2.38$

Wacholder, E. 2.26, 2.27, 2.28

Wagschal, J. J. $4.17,4.18,4.20,4.21,4.22$, 4.24

Walter, G. $\quad 1.20,1.21$

Ward, R. C. $8.39,8.40,8.60,8.62,8.64$

Watts, J. A. $7.4,7.12$

Webster, C. C. 4.19

Weisbin, C. R. 4.1, 4.2, 4.3, 4.4, 4.5, 4.6, 4.7, 7.12 
West, J. T. 7.8

West, K. W. 5.4

Weston, L. W. 1.6, 1.17, 1.23, 1.35

White, J. C. 4.3

White, J. D. 6.4

White, J. E. $3.4,7.5,7.6,7.9,7.10$

White, J. R. $2.39,2.41,2.42,2.43$

Williams, J. 4.22

Williams, M. L. $4.16,4.18,4.19,4.20,4.21$, $4.22,4.23,4.24,4.25,4.28$

Wilson, D. G. $8.6,8.9,8.22,8.23,8.24,8.25$, $8.26,8.29,8.32,8.33,8.34,8.35,8.36,8.37$, 8.38
Wilson, R. 3.9

Winters, R. R. 1.56

Witherspoon, J. P. 4.30

Wolf, J. J. $5.24,5.25,5.26,5.27$

Wood, R. T. $2.25,2.32$

Worley, B. A. $2.19,2.20,2.22,2.23,4.12$, $4.13,4.14,4.24,4.28,4.29,4.33$

Wright, R. Q. $4.28,4.33$

Wright, T. $8.112,8.113,8.117,8.118,8.119$

Yodh, G. B. $3.12,3.13$

Zeighami, E. A. 8.120 


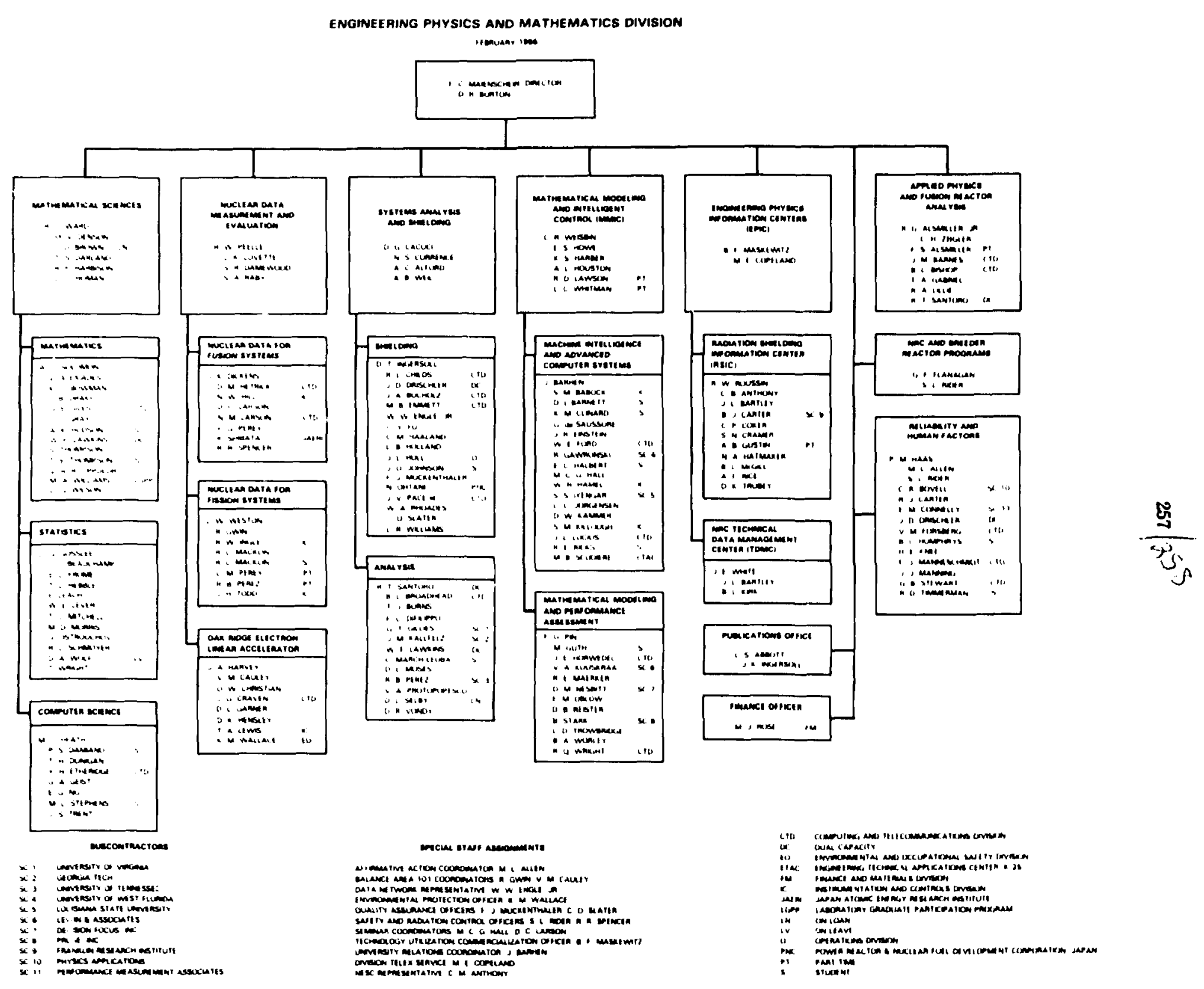




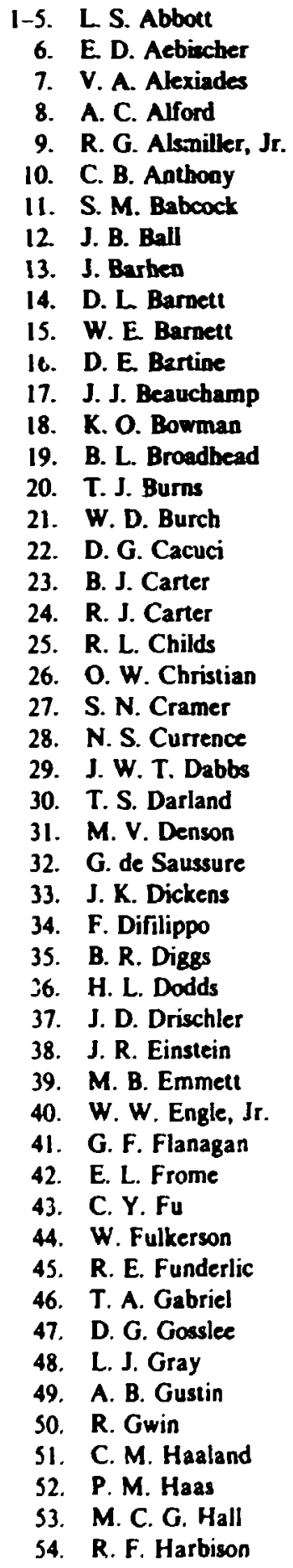

i5. J. A niarvey

56. N. Hatmaker

57. M. T. Heath

58. O. W. Hermann

59. N. W. Hill

60. D. J. Horea

61. E S. Howe

62. A. K. Hudson

63. D. C. Human

64. D. T. Ingersoll

65. J. K. Ingersoll

66. J. O. Johnsoa

67. W. C. Jordan

68. C. C. Josgensen

69. P. R. Kasten

70. S. V. Kaye

71. B. L. Kirk

72. H. E. Knee

73. C. Krause

74. E. H. Krieg

75. P. A. Krois

76. D. C. Larson

77. N. M. Larson

78. E Leach

79. R. P. Leinius

80. W. E. Lever

81. R. A. Lillic

82. L. K. Lovette

83. R. L. Macklin

84. R. E. Macrker

85-109. F. C. Maienschein

110. A. P. Malinauskas

1I1. J. J. Manning

112. B. F. Maskewitz

113. B. L. McGill

114. T. J. Mitchell

115. M. D. Morris

116. F. J. Muckenthaler

117. D. K. Olsen

118. G. Ostrouchov

119. C. V. Parks

120. D. C. Parzyck

121. R. W. Peelle

122. C. M. Perey

123. F. G. Perey

124. R. B. Perez

125. F. G. Pin

126. IS. Postma

127. V. A. Protopopescu

128. S. A. Raby 
129. S. Raman

130. D. Reister

131. R. E. Ricks

132. M. J. Rose

133. R. W. Roussin

134. R. T. Santoro

135. D. L. Selby

136. W. D. Shults

137. E. G. Silver

138. A. D. Solomon

139. J. S. Tang

140. J. L. Thompson

141. S. Thompson

142. R. N. Thurmer

143. R. D. Timmerman

144. J. H. Todd

145. D. K. Trubey

146. V. R. R. Uppuluri

147. D. R. Vondy

148. K. M. Wallace

149. R. C. Ward

150. C. R. Weisbin

151. R. M. Westfall
152. J. E. White

153. G. E. Whitesides

154. T. J. Wilbanks

155. M. K. Wilkinson

156. L. R. Williams

157. D. G. Wilson

158. P. A. Worley

159. R. Q. Wright

160. T. Wright

161. R. G. Wymer

162. C. H. Zeigler

163. A. Zucker

164. P. W. Dickson, Jr. (Consultant)

165. H. J. C. Kouts (Consultant)

166. R. M. Haralick (Consiltant)

167. D. Steiner (Consultant)

168-169. Central Research Library

170. Y-12 Document Ref. Section

171-172. Laboratory Records

173. Laboratory Records ORNL, RC

174. ORNL Patent Offica

175. Program Planning \& Analysis Office

176-189. EPMD Reports Office

\section{EXTERNAL DISTRIBUTION}

190. Office of the Assistant Manager for Energy Research \& Development, DOE-ORO, Oak Ridge, TN 37830.

191. Dr. Anton J. Haug, Manager, ASW Advanced Requirements, Martin Marietta Aerosp :ce, 103 Chesapeake Park Plaza, Baltimore, i.1D 21220

192-193. Technical Information Center

194-250. Given Engineering Physics and Mathematics Division Special Distribution isS.

251-367. Given distribution as shown in TID-4500, Distribution Category UC-34c, Physics - Nuclear. 